\title{
Cool outflows in galaxies and their implications
}

\author{
Sylvain Veilleux ${ }^{1,2,3,4}$, Roberto Maiolino ${ }^{3,5}$, \\ Alberto D. Bolatto ${ }^{1}$, and Susanne Aalto ${ }^{6}$
}

Received: date / Accepted: date

\begin{abstract}
Neutral-atomic and molecular outflows are a common occurrence in galaxies, near and far. They operate over the full extent of their galaxy hosts, from the innermost regions of galactic nuclei to the outermost reaches of galaxy halos. They carry a substantial amount of material that would otherwise have been used to form new stars. These cool outflows may have a profound impact on the evolution of their host galaxies and environments. This article provides an overview of the basic physics of cool outflows, a comprehensive assessment of the observational techniques and diagnostic tools used to characterize them, a detailed description of the best-studied cases, and a more general discussion of the statistical properties of these outflows in the local and distant universe. The remaining outstanding issues that have not yet been resolved are summarized at the end of the review to inspire new research directions.
\end{abstract}

Keywords Galaxies: Active · Evolution · Halo · Kinematics and Dynamics ·

Starburst

\section{Contents}

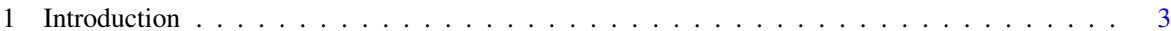

1.1 Setting the Scene . . . . . . . . . . . . . . . . . . . 3

1.2 Scope of the Review, Key Questions, and Organization _ . . . . . . . . . . . . . 4

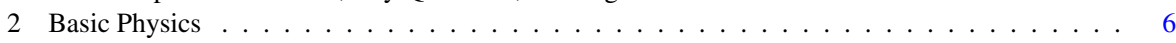

${ }^{1}$ Department of Astronomy and Joint Space-Science Institute, University of Maryland, College Park, MD 20742, USA

2 Institute of Astronomy, University of Cambridge, Cambridge, CB3 0HA, United Kingdom;

3 Kavli Institute for Cosmology Cambridge, University of Cambridge, Cambridge, CB3 OHA, United Kingdom;

${ }^{4}$ Space Telescope Science Institute, Baltimore, MD 21218, USA

${ }^{5}$ Cavendish Laboratory, University of Cambridge, Cambridge, CB3 0HA, United Kingdom;

${ }^{6}$ Department of Earth and Space Sciences, Chalmers University of Technology, Onsala Space Observatory, SE-439 92, Onsala, Sweden

E-mail: Sylvain Veilleux: veilleux@astro.umd.edu, Roberto Maiolino: r.maiolino@mrao.cam.ac.uk, Alberto Bolatto: bolatto@astro.umd.edu, Susanne Aalto: susanne.aalto@chalmers.se 
2.1 Energy Sources . . . . . . . . . . . . . . . . . . . . . 6

2.1.1 Stellar Winds and Supernovae . . . . . . . . . . . . . . . . . 6

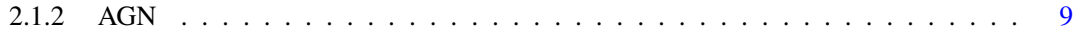

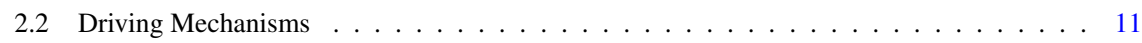

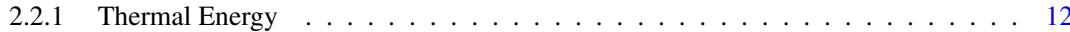

2.2 .2 Radiation . . . . . . . . . . . . . . . . . . . . . . . . . . . . . . . . . . . . . . .

2.2 .3 Cosmic Rays . . . . . . . . . . . . . . . . . . . . . . . . . . 16

2.2 .4 Radio Jets . . . . . . . . . . . . . . . . . . . . . . . . . . 16

2.3 Physical Origin of the Outflowing Cool Gas . . . . . . . . . . . . . . . . . . . . . . . . . . . . . .

2.3.1 Cool Gas Entrainment . . . . . . . . . . . . . . . . . . 17

2.3.2 In-Situ Formation from the Hot Wind Material _ . . . . . . . . . . . . . . . . . . . 19

2.4 Minimum Requirement for the Outflowing Material to Escape the Host Galaxy . . . . . . 22

3 Observational Techniques and their Limitations . . . . . . . . . . . . . . . . . . . . . 22

3.1 Kinematic Definition of an Outflow . . . . . . . . . . . . . . . . . . . 23

3.1 .1 Spatially Unresolved Outflows . . . . . . . . . . . . . . . . . . 23

3.1.2 Spatially Resolved Outflows . . . . . . . . . . . . . . . . . . 23

3.2 Derivation of Outflow Energetics: Basic Formulae . . . . . . . . . . . . . . . . . . . . . . . . . . . . . . . . .

3.3 Diagnostic Tools of the Neutral-Atomic Gas Phase . . . . . . . . . . . . . . . . . . . . . . . . . 27

3.3 .1 H I $21 \mathrm{~cm} \ldots \ldots \ldots \ldots 27$

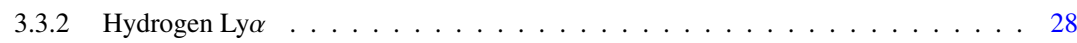

3.3 .3 Na I D $\lambda \lambda 5890,5896 \ldots \ldots \ldots \ldots$

3.3.4 UV Lines from Neutral Elements . . . . . . . . . . . . . . . . . . 33

3.3.5 UV lines from Singly Ionized Elements . . . . . . . . . . . . . . . . . . . 34

3.3 .6 Far-Infrared $[\mathrm{C}$ I $]$ and $[\mathrm{C}$ II $]$ Emission Lines . . . . . . . . . . . . . . . . . . . . . . 35

3.4 Diagnostic Tools of the Molecular Gas Phase . . . . . . . . . . . . . . . . . . . . . . . . . . . . . . . . . .

3.4.1 Probes of the Volume Density . . . . . . . . . . . . . . . . . . . 37

3.4.2 Probes of the Column Density and Optical Depth . . . . . . . . . . . . . . . . . 38

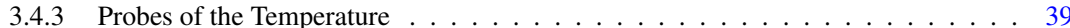

3.4.4 Estimating the Masses and Mass-Outflow Rates . . . . . . . . . . . . . . . . . . . . . 40

3.4.5 Importance of Shocks, X-rays, and Cosmic Rays . . . . . . . . . . . . . . . . 45

3.4 .6 Compact Obscured Nuclei: CONs . . . . . . . . . . . . . . . . . . . . 46

3.4 .7 Direct $\mathrm{H}_{2}$ Probes . . . . . . . . . . . . . . . . . . . . . . . 47

3.5 Diagnostic Tools of the Dust . . . . . . . . . . . . . . . . . . . . . . 48

3.5.1 Extinction and Reddening . . . . . . . . . . . . . . . . . . . . 49

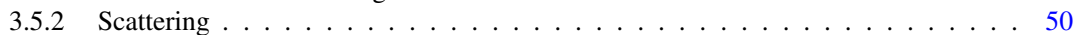

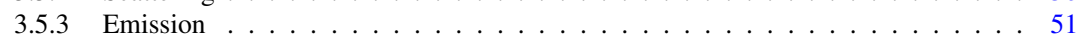

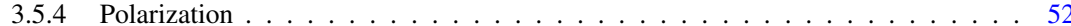

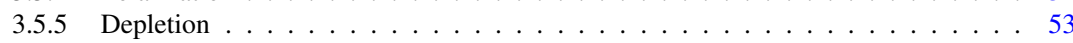

3.5.6 Dust Cycle: Formation and Destruction . . . . . . . . . . . . . . . 53

4 Best-Studied Cases of Cool Outflows . . . . . . . . . . . . . . . . . . . . . . 54

4.1 The Milky Way and the Magellanic Clouds . . . . . . . . . . . . . . . . . . . . . 54

4.1 .1 The Milky Way . . . . . . . . . . . . . . . . . . . . . 54

4.1 .2 The Magellanic Clouds . . . . . . . . . . . . . . . . . . . . . . 58

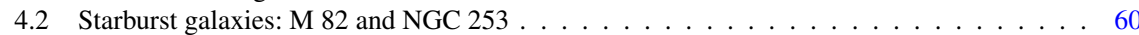

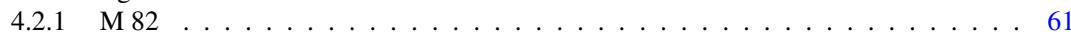

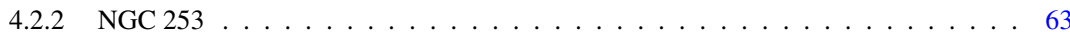

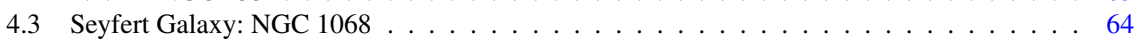

4.4 Quasar: Mrk $231 \ldots \ldots \ldots \ldots \ldots \ldots$

4.5 Jetted AGN: Centaurus A, IC 5063, NGC 1266, and NGC 1377 . . . . . . . . . . . . . 69

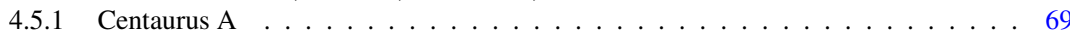

4.5 .2 IC 5063 and NGC $1266 \ldots \ldots \ldots \ldots \ldots \ldots$

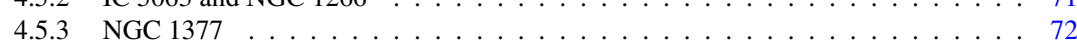

5 Cool Outflows in the Local $(z \lesssim 1)$ Universe . . . . . . . . . . . . . . . . . . . . . . . . . 74

5.1 Neutral Atomic Gas Component . . . . . . . . . . . . . . . . . . . . . . . . 74

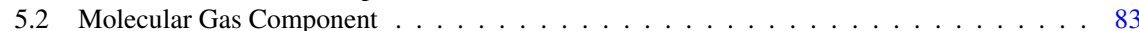

5.3 Dust Component . . . . . . . . . . . . . . . . . . . . . . . 90

5.4 Driving Mechanisms in AGN-dominated Systems . . . . . . . . . . . . . . . . . 96

5.5 Compact Obscured Nuclei (CONs): A Special Evolutionary Phase? . . . . . . . . . . . . . 99 
5.6 Fate of the Outflowing Material . . . . . . . . . . . . . . . . . . . 101

6 Cool Outflows in the Distant Universe . . . . . . . . . . . . . . . . . . . . . . . . 105

6.1 Molecular Gas Component . . . . . . . . . . . . . . . . . . . . . . . . 105

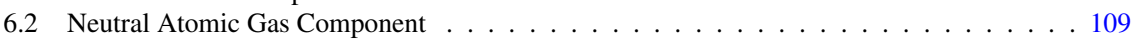

6.3 Dust Component . . . . . . . . . . . . . . . . . . . . . . . . . . 113

6.4 Fate of the Outflowing Material . . . . . . . . . . . . . . . . . . . 114

7 Open Issues and Future Directions . . . . . . . . . . . . . . . . . . . . . 115

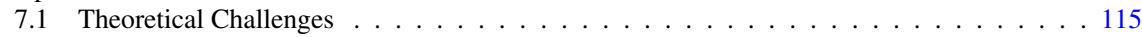

7.1.1 Wind-ISM Interaction . . . . . . . . . . . . . . . . . . . 115

7.1.2 Production and Survival of Dust and Molecules . . . . . . . . . . . . . . . . 116

7.1.3 Coupling the Radiation to the Cool Gas . . . . . . . . . . . . . . . . . 117

7.1.4 Importance of Cosmic Rays . . . . . . . . . . . . . . . . . . . . . . . . . . . . 117

7.2 Observational Challenges . . . . . . . . . . . . . . . . . 118

7.2.1 Unbiased Census of Local Molecular Outflows . . . . . . . . . . . . . . . . . . . 118

7.2.2 Outflow Physical Properties . . . . . . . . . . . . . . . . . . . 118

7.2.3 Negative Feedback: Zone of Influence and Escape Fraction . . . . . . . . . . . . . 119

7.2.4 Positive Feedback: Induced and In-Situ Star Formation . . . . . . . . . . . . . . . 119

7.2 .5 Evolution with Look-Back Time . . . . . . . . . . . . . . . . . . . . . . . . . . . . 120

\section{Introduction}

\subsection{Setting the Scene}

Galaxies are not isolated "Island Universes" the way Thomas Wright and Immanuel Kant imagined them (Wright 1750; Kant 1755). Instead, they are giant ecosystems where material flows in and out or is processed in-situ. The fate of baryons in a galaxy is governed by the complex interplay between gas entering the galaxy through galaxy mergers and accretion flows, star formation within the galaxy, and gas flowing out of the galaxy. All gas phases and dust are participating in this cosmic ballet, but the focus of this review is on the cool $\left(T \lesssim 10^{4} \mathrm{~K}\right)$ outflowing neutral-atomic, molecular, and dusty material (Fig. 1). Driven by the energy released from stellar processes and gas accretion onto supermassive black holes (SMBHs) at the centers of galaxies, these cool outflows are a common feature of local and distant gas-rich systems. They often dominate the mass, and also sometimes the energetics, of gas outflows in general, and may comprise a significant fraction of the entire reservoir of cool gas in the galaxy hosts.

The study of cool outflows is a relatively new area of research, dating back $\lesssim$ 20 years. However, in the last decade, thanks in large part to the advent of new infrared space observatories (Spitzer, Herschel), new or upgraded mm-wave and cmwave ground-based facilities (ALMA, NOEMA, VLA, and GBT), and a suite of newgeneration optical and near-infrared multi-object and integral field spectrometers on large ground-based telescopes (e.g., SAMI, KMOS, MaNGA, MOSFIRE, MUSE, $\mathrm{KCWI}$ ), the study of cool outflows has flourished into a full-fledged sub-discipline of its own with implications that touch on vast areas of extragalactic astronomy including galaxy formation and evolution and the synergistic connection between galaxies and supermassive black holes.

Cool outflows have deservedly received considerable attention in recent years for a number of reasons. The cool gas taking part in these outflows is the raw material from which stars are formed, so its fate may affect the evolution of the host galaxies. 
Fast outflows are among the leading internal negative-feedback processes to explain the rapid ( $\lesssim 10^{9} \mathrm{yrs}$ ) inside-out cessation ("quenching") of star formation in massive galaxies (Schawinski et al. 2014; Onodera et al. 2015; Tacchella et al. 2015, 2016; Spilker et al. 2019). However, simply carrying the cool gas out of the galaxy and depositing it into the circumgalactic medium (CGM; Tumlinson et al. 2017) may not be sufficient to quench these galaxies since the cool material, if left alone, will eventually fall back onto the galaxy and form new stars. Even if the outflows are powerful enough to eject the gas out into the intergalactic medium (IGM; Oppenheimer et al. 2019; Davies et al. 2019; Keller et al. 2019), re-accretion of the enriched material ejected by neighboring galaxies may revive the star formation activity of the host galaxy (Anglés-Alcázar et al. 2017). The duty cycle of outflows must be fine-tuned to prevent this material from infalling back onto the galaxy (e.g., Kim and Ostriker 2018), or other processes must be at work to prevent it from forming new stars (Faerman et al. 2017; McQuinn and Werk 2018).

Powerful winds, driven by a central quasar or the surrounding starburst, have also been invoked to stop the growth of both the $\mathrm{BH}$ and spheroid component (Silk and Rees 1998; Fabian 1999; King 2003; Murray et al. 2005) and explain the tight BHspheroid mass relation (e.g., Magorrian et al. 1998; Gebhardt et al. 2000; Ferrarese and Merritt 2000). In turn, the fraction of quenched galaxies in the local universe seems strongly correlated with the properties of the spheroid component (Teimoorinia et al. 2016; Bluck et al. 2016, 2019), pointing back to BH-driven outflows as a means to quench massive galaxies. Fast AGN-driven cool outflows may be examples of this process in action.

Much of our understanding of the overall impact of galactic winds and outflows on galaxy evolution comes from sophisticated cosmological galaxy formation simulations that incorporate the observable properties of the best-studied outflow systems (Vogelsberger et al. 2014; Schaye et al. 2015; Dubois et al. 2016; Choi et al. 2018; Biernacki and Teyssier 2018; Brennan et al. 2018; Hopkins et al. 2018b; Davé et al. 2019; Nelson et al. 2019; Peeples et al. 2019; Hafen et al. 2019). However, the cool gas phase outside of galaxies is notoriously difficult to model since it is subject to formation and destruction processes that act on scales that are unresolved in these simulations (e.g., thermal, Kelvin-Helmholtz, and Rayleigh-Taylor instabilities, turbulent boundary layers, conductive heat transport). We therefore expect new data on cool gas outflows to continue to inform the next generation of numerical simulations of galaxy formation and evolution for years to come.

\subsection{Scope of the Review, Key Questions, and Organization}

The focus of this review is on cool $\left(T \lesssim 10^{4} \mathrm{~K}\right)$ outflows comprised of neutralatomic and molecular gas where hydrogen is mostly in neutral or molecular form, respectively. Dust is also part of our discussion since in general a significant fraction (perhaps 30\%; Weingartner and Draine 2001) of the metals in cool enriched gas is locked into dust. Dust grains are an essential component of these outflows, acting as anchor points for radiation forces and catalyzers for $\mathrm{H}_{2}$ formation. This review is meant to provide a holistic view of cool outflows, spanning the full range of scales 

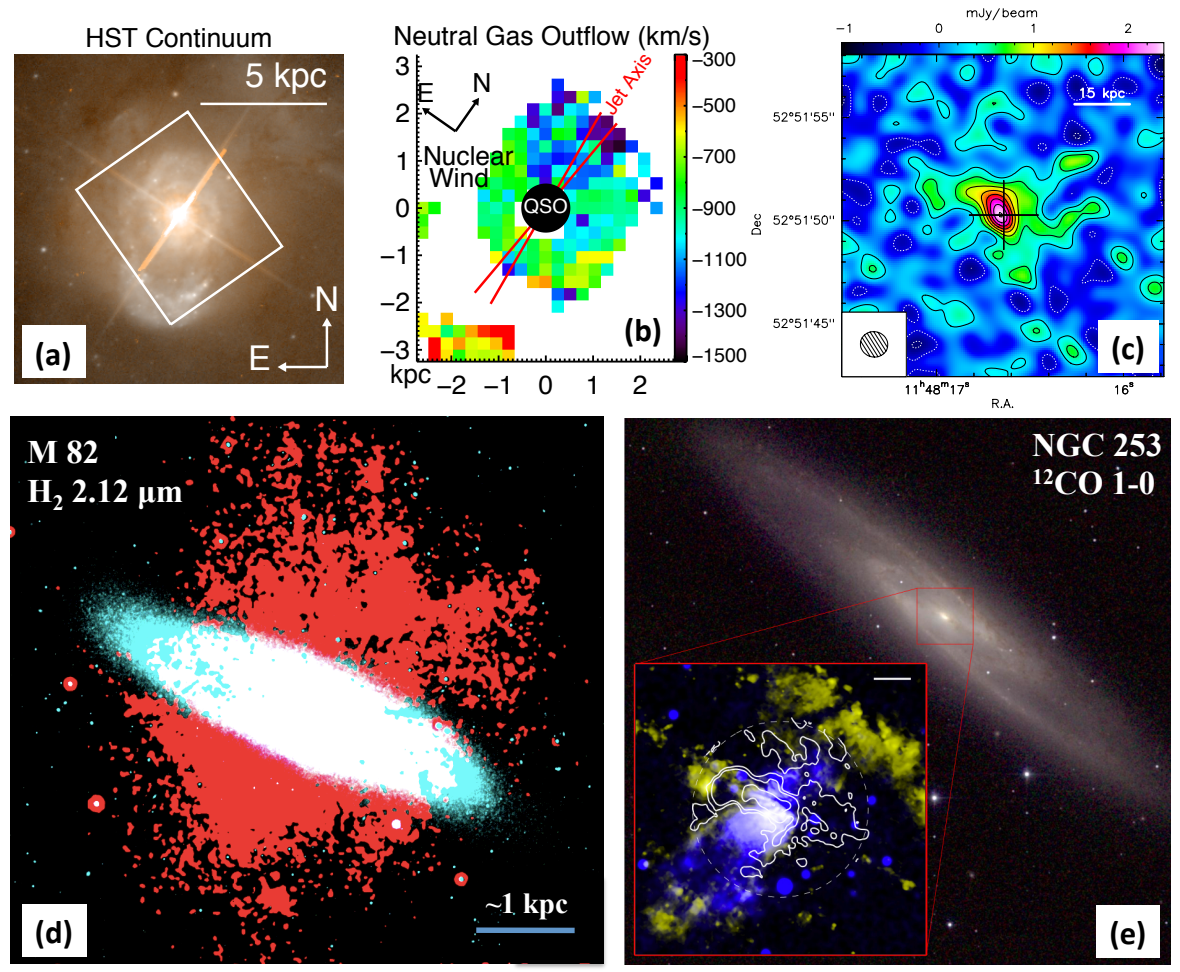

Fig. 1 Examples of cool outflows, near and far. (a) HST optical continuum image of quasar Mrk 231. The white box is the footprint of the IFS data shown in panel (b). (b) Velocity field of Na I D absorption line in Mrk 231. (c) [C II] $158 \mu \mathrm{m}$ line emission in $z=6.4$ quasar SDSS J1148+5251. (d) Starburst galaxy M 82 in $\mathrm{H}_{2} 2.12 \mu \mathrm{m}$ (red) and stellar light (light blue). (e) Starburst galaxy NGC 253 in CO 1-0 (white contours) relative to $\mathrm{H} \alpha$ (yet), X-rays (blue), and optical continuum emission (greyscale). Images reproduced with permission from (a, b) Rupke and Veilleux (2011), (d) Veilleux et al. (2009b), copyright by AAS; and (c) Cicone et al. (2015), copyright by ESO; and (e) Bolatto et al. (2013a), copyright by Macmillan.

over which they are observed, i.e. from $\lesssim 10 \mathrm{pc}$ in some AGN and quasars to $\gtrsim$ $100 \mathrm{kpc}$ in some distant galaxies. Most of our discussion centers on the following fundamental questions:

- Statistical properties of cool winds in the local and distant universe: What is their frequency of occurrence, physical extent, mass loss rate, efficiency, energetics, and evolution with look-back time?

- Driving mechanisms of cool winds: What is the role of supernovae, radiation pressure, cosmic-ray pressure, and AGN? Is there a minimum threshold of star formation or black hole activity needed to trigger cool outflows? How is the material entrained but not destroyed?

- Multi-scale and multi-phase nature of outflows: What is the relative distribution of the cool, warm, and hot phases in the wind? Is the cool material produced insitu from the hot wind? Is the cool material transitioning to another gas phase on 
the way out? Do cool winds form stars? What is the relation between large-scale cool outflows and nuclear AGN winds?

- Impact of cool winds on the host galaxy: What feedback effects do they exert on the host galaxy ISM and CGM? Do they help regulate the growth of the black hole? What fraction of the cool outflowing gas escapes the host galaxy? What is the role of re-accreted (recycled) gas from neighboring galaxies?

This review is organized as follows. Section 2 describes the basic physics behind cool outflows, particularly the sources of energy and momentum, driving mechanisms, physical origin of the outflowing cool gas, and minimum condition for the outflowing material to escape galaxies. Section 3 discusses the many observational techniques and diagnostic tools that have been used so far to measure the properties of cool outflows, and provides a critical assessment of their strengths and weaknesses. The best-studied cases of cool outflows are described in detail in Section 4 to set the stage for a more general discussion of the statistical properties of cool outflows in the nearby and distant universe in Sections 5 and 6, respectively. This review concludes in Section 7 with a summary of the key open theoretical and observational issues, and suggestions for future research directions.

This type of review is necessarily incomplete and biased. While our main target audience is newcomers who wish to familiarize themselves with this topic or may be searching for exciting new research ideas, we hope that this review will also be a valuable resource for more experienced scientists of all levels. Those who did not find an answer to their questions in this article may wish to consult the following recent reviews on directly relevant topics: Erb (2015, stellar feedback in low-mass galaxies), King and Pounds (2015, AGN outflows and feedback), Harrison (2017, AGN feedback), Heckman and Thompson (2017, galactic winds in general), Tumlinson et al. (2017, CGM), Harrison et al. (2018, AGN outflows and feedback), Rupke (2018, galactic winds driven by star formation), Cicone et al. (2018a, multiphase outflows in AGN host galaxies), Wylezalek and Morganti (2018, the driving mechanisms of outflows in AGN), Morganti and Oosterloo (2018, HI 21-cm absorption as a probe of the ISM and CGM in AGN), and Zhang et al. (2018, the theory of galactic winds driven by stellar feedback). The earlier reviews by Veilleux et al. (2005, galactic winds in general) and Fabian (2012, observations of AGN feedback) may also be useful. Finally, reviews on more specific topics are mentioned throughout the text.

\section{Basic Physics}

\subsection{Energy Sources}

\subsubsection{Stellar Winds and Supernovae}

Stars inject mass, momentum, and energy in the surrounding environment that may prevent molecular gas from forming stars on a dynamical time scale. Given a star formation rate $\dot{M}_{*}$, the depletion time scale $t_{\text {dep }} \equiv M_{\text {gas }} / \dot{M}_{*}$ of the molecular gas in galaxies due to star formation is typically $\sim 1-2 \mathrm{Gyr}$ on $\gtrsim \mathrm{kpc}$ scales (e.g., Bigiel et al. 2008), although $t_{\text {dep }}$ tends to decrease with increasing specific star formation 
rates, both spatially integrated $\left(\dot{M}_{*} / M_{*} ;\right.$ Saintonge et al. 2011) and spatially resolved ( $\Sigma_{\mathrm{SFR}} / \Sigma_{*}$; Ellison et al. 2019), and there is considerable scatter on scales below $\sim 100$ pc (Kennicutt and Evans 2012; Kruijssen and Longmore 2014). This depletion time scale is roughly two orders of magnitude longer than the dynamical time scale of giant molecular clouds (GMCs): $t_{\mathrm{ff}} / t_{\mathrm{dep}} \equiv \epsilon_{\mathrm{ff}} \simeq 1 \%$, where $t_{\mathrm{ff}}=\sqrt{3 \pi / 32 G \rho}$ is the local free-fall time and $\epsilon_{\mathrm{ff}}$ is the star formation efficiency per free-fall time. Recently, Kruijssen et al. (2019) have deduced GMC lifetimes of $t_{\mathrm{sf}} \simeq 11 \mathrm{Myr}$ resulting in a star formation efficiency per GMC lifetime $\epsilon_{\mathrm{Sf}}=2.5 \%$. A wide range of feedback processes may be responsible for this low star formation efficiency. Since most stars form in clusters, the emphasis of our discussion is on the dominant feedback processes in cluster environments.

- Protostellar Outflows. This source of stellar feedback has been reviewed in detailed in Bally (2016). It is clear that protostellar outflows help keep $\epsilon_{\mathrm{ff}}$ small in the dense regions of star clusters, but given the low ejection velocities, these outflows play a limited role in setting the final value of $\epsilon_{\mathrm{ff}}$ in most clusters, except those with masses less than $\sim 100 M_{\odot}$ (Krumholz et al. 2019).

- Stellar Winds from Young Massive Stars. Winds from massive supernova (SN) progenitor stars profoundly transform the circumstellar medium around these stars (e.g., van Marle and Keppens 2012; Georgy et al. 2013), and also likely play a significant role in clearing the denser gas from young stellar clusters, before the first corecollapse SNe occur (Longmore et al. 2014; Hollyhead et al. 2015; Sokal et al. 2016). Pre-existing wind bubbles at the time of the SN explosion may greatly enhance the feedback energy efficiency, although the exact boost depends on the uncertain importance of mixing, conduction, and cooling at the interface of the hot wind bubble and the compressed swept-up ambient medium (e.g., Fierlinger et al. 2016; El-Badry et al. 2019). Pre-existing wind-blown bubbles created by massive SN progenitor stars may also affect the survival or destruction of the dust generated within the ejecta or initially present in the ambient ISM (Martínez-González et al. 2019).

- Core Collapse Supernovae. Stars with masses between about 8 and $25 \mathrm{M}_{\odot}$ end their lives after $\sim 3 \mathrm{Myr}$ as core collapse supernovae ejecting $\sim 1-10 M_{\odot}$ of metalenriched material with kinetic energy $E_{\mathrm{SN}} \simeq 10^{51} \mathrm{erg} \equiv E_{51}$ and momentum $p_{\mathrm{SN}}=$ $\sqrt{2 E_{\mathrm{SN}} M_{\mathrm{SN}}}$, where $M_{\mathrm{SN}}$ is the mass of the ejecta per SN. If the supernova rate $\Gamma_{\mathrm{SN}}$ scales with the star formation rate as $\Gamma_{\mathrm{SN}}=\alpha_{\mathrm{SN}} \dot{M}_{*}$, where $\alpha_{\mathrm{SN}} \simeq 0.01-0.02$ (depending on the Initial Mass Function, IMF; $\alpha_{\mathrm{SN}} \simeq 0.02$ for a Salpeter IMF and continuous star formation; Leitherer et al. 1999), then $\dot{E}_{\mathrm{SN}}=7 \times 10^{41}\left(\alpha_{\mathrm{SN}} / 0.02\right)\left(\dot{M}_{*} / M_{\odot} \mathrm{yr}^{-1}\right)$ $\mathrm{erg} \mathrm{s}^{-1}$ and $\dot{p}_{\mathrm{SN}}=5 \times 10^{33}\left(\alpha_{\mathrm{SN}} / 0.02\right)\left(\dot{M}_{*} / M_{\odot} \mathrm{yr}^{-1}\right)$ dyne (Veilleux et al. 2005). What happens next to this kinetic energy depends on the local pre-SN environment. In very low density environments, the ejecta simply remain in a state of free expansion keeping the same kinetic energy and momentum. In more typical environments, some fraction of the kinetic energy ( $\xi$, the supernova thermalization or feedback efficiency) is quickly transformed into thermal energy through shocks heating the SN ejecta and ambient medium to a temperature $T=0.4 \mu m_{H}(\dot{E} / k \dot{M}) \simeq 3 \times 10^{8} \xi \Lambda^{-1}$ $\mathrm{K}$, where $\Lambda$ is the ratio of the total mass of heated gas to the mass that is directly ejected by the SN. Once the mass of swept up material is comparable to the mass of the ejecta, the SN remnant enters an adiabatic Sedov-Taylor phase of expansion 
where the outer shock radius expands as $t^{2 / 5}$. Once the temperature drops below $\sim 10^{6}$ $\mathrm{K}$, radiative losses become important, and the $\mathrm{SN}$ remnant enters a pressure-driven snowplow phase where the pressure of the interior hot gas is still larger than that of the outside pressure. Gradually, the interior pressure decreases to eventually match the outside pressure, and the remnant continues to expand and sweep up ISM gas as a momentum-conserving snowplow. Several studies have shown that the input momentum from SNe can be boosted by an order of magnitude during the Sedov-Taylor phase due to work done by the hot shocked gas (e.g., Kim and Ostriker 2015; Kimm et al. 2015; Martizzi et al. 2015; Walch and Naab 2015): $p_{\mathrm{SN}}=3 \times 10^{5} \mathrm{~km} \mathrm{~s}^{-1}$ $\mathrm{M}_{\odot} E_{51}^{16 / 17} n_{\mathrm{H}}^{-2 / 17} Z^{-0.14}$, where $n_{\mathrm{H}}$ is the hydrogen number density in $\mathrm{cm}^{-3}$ and $Z$ is the metallicity in solar units (formally this expression is valid only if $Z \geq 0.01$ ). Note that $p_{\mathrm{SN}}$ depends only weakly on the density (or density inhomogeneity) of the ambient medium and its metallicity. Considerable effort has been invested in recent years in determining the impact of clustered supernovae on this momentum boost. It has been found that the asymptotic momentum per SN can be up to an order of magnitude greater than that delivered by isolated $\mathrm{SNe}$ (up to $\sim 3 \times 10^{6} M_{\odot} \mathrm{km} \mathrm{s}^{-1}$ per $\mathrm{SN}$ ), although the results are sensitive once again on the mixing rate across the contact discontinuity between the hot and cold phases (Sharma et al. 2014; Gentry et al. 2017, 2019; El-Badry et al. 2019).

- Cosmic Rays. In star-forming galaxies like the Milky Way, first-order Fermi shock accelerated particles in supernova remnants are the dominant source of cosmic rays (CRs; e.g., Grenier et al. 2015; Bykov et al. 2018). These cosmic rays ionize and heat the ISM, including the cold material that is optically thick to ionizing radiation, depositing $\sim 13 \mathrm{eV}$ per Coulombic or hadronic interaction (e.g., Grenier et al. 2015). Between 10 and $50 \%$ of the kinetic energy from SNe $\left(\sim 1-5 \times 10^{50}\right.$ ergs per SN) can be converted into non-thermal energy (Grenier et al. 2015; Diesing and Caprioli 2018), most of which is deposited in protons with energies following a power-law distribution. In the case of a Salpeter IMF and constant star formation rate, the CR energy injection rate is thus $\dot{E}_{\mathrm{CR}} \simeq(0.7-3.5) \times 10^{41}\left(\alpha_{\mathrm{SN}} / 0.02\right)\left(\dot{M}_{*} / M_{\odot} \mathrm{yr}^{-1}\right) \mathrm{erg} \mathrm{s}^{-1}$ and the injected momentum rate is $\dot{p}_{\mathrm{CR}}=(0.5-2.5) \times 10^{33}\left(\alpha_{\mathrm{SN}} / 0.02\right)\left(\dot{M}_{*} / M_{\odot} \mathrm{yr}^{-1}\right)$ dyne. This last number does not take into account the possibility that the asymptotic injected momentum per SN may be boosted by the presence of CRs by a factor of 2-3, and perhaps as much as an order of magnitude in the denser environments. This is due to the fact that CRs act as a relativistic fluid, so they suffer less adiabatic loss than the thermal gas, and thus dominate the internal pressure at late times in the evolution of the SN remnant. Moreover, the CR energy is not radiated away during the snowplow phase, so it continues to support the expansion of the SN remnant (Diesing and Caprioli 2018).

- Radiation. The stellar radiative processes most relevant to cool outflows are photoionization heating associated with the ionizing $(h v>13.6 \mathrm{eV})$ radiation from hot stars and $\mathrm{SNe}$, photo-electric heating associated with the non-ionizing radiation absorbed by dust in the warm and cold neutral media, Compton heating by hard photons from X-ray binaries, the dissociation of $\mathrm{H}_{2}$ by the Lyman-Werner (11.2-13.6 $\mathrm{eV}$ ) radiation, and finally the radiation pressure associated with single- and multiplescattering processes. Most of these processes provide excellent diagnostic tools to 
study cool outflows so they are discussed in more details in Section 3. Radiation pressure is potentially an important driving mechanism for cool outflows so it is discussed in more detail in Section 2.2.2. For starbursts, the luminosity is typically $L \simeq 10^{10} \dot{M}_{*} L_{\odot}$, where the units of the star formation rate $\dot{M}_{*}$ are $M_{\odot} \mathrm{yr}^{-1}$.

- Type Ia Supernovae. Stars with $M<8 \mathrm{M}_{\odot}$ are the progenitors of Type Ia supernovae that go off after $\sim 100 \mathrm{Myr}$, each injecting an energy $\sim 10^{51}$ erg and a mass $\sim 1.4 \mathrm{M}_{\odot}$ into the surrounding environment. The energy deposition rate from a galaxy with stellar mass $M_{*, 11}$ in units of $10^{11} M_{\odot}$ and stellar population age $t_{9.7}$ in units of 5 $\times 10^{9} \mathrm{yrs}$ is $\dot{E}_{I a} \simeq 14 \times 10^{40} M_{*, 11} t_{9.7}^{-1.1} \mathrm{erg} \mathrm{s}^{-1}$ (Conroy et al. 2015). This source of energy may play a role in preventing star formation in low-mass early-type galaxies (e.g. Li et al. 2018), but is probably not an important contributor to the energetics of the cool outflows reported here in star-forming and active galaxies.

- Stellar Winds from Old Stars. The thermalization of winds from asymptotic giant branch (AGB) stars, red giants, and planetary nebula phases injects energy at a rate $\dot{E}_{A G B} \simeq 5 \times 10^{40} M_{*, 11} \sigma_{*, 300}^{2} t_{9.7}^{-1.25} \mathrm{erg} \mathrm{s}^{-1}$ (Conroy et al. 2015), where $\sigma_{*, 300}$ is the stellar velocity dispersion of the host galaxy normalized to $300 \mathrm{~km} \mathrm{~s}^{-1}$. This energy will combine with that injected from Type Ia SNe (discussed above), but does not play a role in driving the cool outflows in starburst and active galaxies.

\subsubsection{AGN}

The primary energy source behind AGN is accretion onto the central supermassive black hole, where the gravitational potential energy lost by the accreted material is converted into heat and partly radiated away at the rate of

$$
L=\epsilon_{r} \dot{M}_{\mathrm{acc}} c^{2} \simeq 5.7 \times 10^{45}\left(\epsilon_{r} / 0.1\right)\left(\dot{M}_{\mathrm{acc}} / M_{\odot} \mathrm{yr}^{-1}\right) \mathrm{erg} \mathrm{s}^{-1},
$$

where $\epsilon_{r}$ is the radiative efficiency. This efficiency is a strong function of the accretion rate. At low accretion rates $\left(\dot{m}_{\mathrm{acc}} \equiv \dot{M}_{\mathrm{acc}} / \dot{M}_{\mathrm{acc}}^{\mathrm{Edd}} \equiv L / L_{\mathrm{Edd}} \lesssim 10^{-3}\right.$, where $L_{\text {Edd }}=4 \pi G M c / \kappa_{e s}=1.25\left(M / M_{\odot}\right) \times 10^{38} \mathrm{erg} \mathrm{s}^{-1}$ is the Eddington luminosity due to electron scattering), the accreting material forms a hot, optically thin, geometrically thick disk which is radiatively inefficient with $\epsilon_{r}<10^{-3}$ because of long cooling time scales (e.g., Yuan and Narayan 2014). At moderate accretion rates $\left(\dot{m}_{\mathrm{acc}} \simeq 0.01-\right.$ 0.25 ), the accretion flow forms a cold (relative to the virial temperature), optically thick, but geometrically thin disk that is radiatively efficient with $\epsilon_{r}\left(a_{\mathrm{BH}}\right) \simeq 0.05-$ 0.2 , increasing with increasing black hole spin $a_{\mathrm{BH}}$ (e.g., Novikov and Thorne 1973; Koratkar and Blaes 1999). Above this accretion rate, long radiative diffusion time scales produce an optically and geometrically thick "slim" disk that is radiatively inefficient with $\epsilon_{r} \lesssim 10^{-3}$ (e.g., Sadowski et al. 2013, 2014; Sadowski and Narayan 2015; Jiang et al. 2014; McKinney et al. 2014, 2015). The relative importance of the dominant launching mechanisms for outflows in AGN - radiation, thermal pressure, non-thermal pressure (from cosmic rays), and magnetic forces - also varies with the mass accretion rate (e.g., Giustini and Proga 2019).

- Radiative Mode of AGN Feedback. For the radiatively efficient AGN, the mechanical energy injection rate in the so-called "radiative" or "quasar" mode of AGN feedback 
may be written

$$
\dot{E}_{r}=\epsilon_{f, r} \epsilon_{r} \dot{M}_{\mathrm{acc}} c^{2} \simeq 5.7 \times 10^{45} \epsilon_{f, r}\left(\epsilon_{r} / 0.1\right)\left(\dot{M}_{\mathrm{acc}} / M_{\odot} \mathrm{yr}^{-1}\right) \mathrm{erg} \mathrm{s}^{-1},
$$

where $\epsilon_{f, r}$ is the efficiency of the radiative energy to couple with the local environment and drive the outflow. Note that this expression implies that the total amount of injected energy over the lifetime of the black hole is

$$
E_{r}=\epsilon_{f, r} \epsilon_{r} M_{\mathrm{BH}} c^{2} \simeq 2 \times 10^{61} \epsilon_{f, r}\left(\epsilon_{r} / 0.1\right)\left(M_{\mathrm{BH}} / 10^{8} M_{\odot}\right) \mathrm{erg} .
$$

This number is two orders of magnitude larger than the binding energy of the bulge hosting the black hole

$$
E_{\text {bulge }} \simeq M_{\text {bulge }} \sigma_{*}^{2} \simeq 2 \times 10^{59}\left(M_{\text {bulge }} / 10^{11} M_{\odot}\right)\left(\sigma_{*} / 300 \mathrm{~km} \mathrm{~s}^{-1}\right)^{2} \mathrm{erg} .
$$

This fact alone implies $\left\langle\epsilon_{f, r}\right\rangle \lesssim 1 \%$, averaged over the active life cycle of the black hole. This number is not well constrained from numerical simulations, although values of $0.5 \%-1 \%$ seem to be required if AGN feedback is to suppress star formation in the host (e.g., Hopkins and Elvis 2010; Hopkins et al. 2016).

- Kinetic Mode of AGN Feedback. For the radiatively inefficient AGN, the mechanical energy injection rate in the "kinetic" or "radio" mode of AGN feedback may be expressed as

$$
\dot{E}_{k}=\epsilon_{f, k} \dot{M}_{\mathrm{acc}} c^{2} \simeq 5.7 \times 10^{46} \epsilon_{f, k}\left(\dot{M}_{\mathrm{acc}} / M_{\odot} \mathrm{yr}^{-1}\right) \mathrm{erg} \mathrm{s}^{-1},
$$

where $\epsilon_{f, k}$ is the efficiency of the kinetic energy injected by the AGN to couple to the local environment and drive the outflow. This kinetic energy may come from both light relativistic jets and slower but more massive wide-angle winds.

Recent simulations of hot accretion flows give $\epsilon_{f, k} \sim 10^{-3}$ (e.g., Yuan et al. 2015). This number includes the contribution from relativistic jets accelerated by processes associated with the magnetized accretion disk (e.g., Blandford and Payne 1982; Lynden-Bell 2003, 2006; Hawley and Krolik 2006), but may be larger in cases where the kinetic energy is delivered by jets produced by the Blandford-Znajek mechanism (Blandford and Znajek 1977). In these objects, the jet power is extracted from the black hole spin and $L_{\mathrm{jet}} \propto B_{\mathrm{pol}}^{2} M_{\mathrm{BH}}^{2} a_{\mathrm{BH}}^{2}$, where $B_{\mathrm{pol}}$ is the poloidal magnetic field at the black hole horizon and $a_{\mathrm{BH}}$ is the black hole spin $\left(a_{\mathrm{BH}}=0.998\right.$ for maximally spinning black holes; Thorne 1974; Yuan and Narayan 2014). Recent slim disk simulations with non-spinning black holes have shown that outflows are inevitable, resulting in $\epsilon_{f, k} \simeq 3 \%$, and as high as $\sim 8 \%$ with spinning black holes with $a_{\mathrm{BH}}=0.7$ (strickly speaking, these numbers include both the radiative and wind energy outputs so they correspond to $\epsilon_{f} \epsilon_{r}+\epsilon_{f, k}$ in our nomenclature; e.g., Sadowski et al. 2016; Sadowski and Narayan 2016).

- Cosmic Rays. In analogy with supernova-driven shocks, the interaction of the aforementioned wide-angle sub-relativistic winds and collimated relativistic jets with the ambient ISM may be an important source of CRs. Protons with energies $>8,21$, and $140 \mathrm{MeV}$ penetrate column densities $N_{H}$ of $2 \times 10^{22} \mathrm{~cm}^{-2}, 1.2 \times 10^{23} \mathrm{~cm}^{-2}$, and 4 $\times 10^{24} \mathrm{~cm}^{-2}$, respectively, and therefore can contribute to heating and ionizing some 
of the material in cool outflows (González-Alfonso et al. 2018). Depending on the details of CR propagation through the ISM, these CRs may also provide a significant source of pressure that will help drive the large-scale outflows (e.g., Chan et al. 2019; Yusef-Zadeh et al. 2019, Sec. 2.2.3). The most extreme ultra-high-energy CRs (UHECRs) with energies $>10^{9} \mathrm{GeV}$ collide with nuclei in the ISM and produce pions that decay into $\mathrm{GeV}-\mathrm{TeV} \gamma$-rays as well as PeV muonic neutrinos (e.g., Wang and Loeb 2016, 2017; Liu et al. 2018).

- Source Variability. The radiative and non-radiative energy output of AGN may vary wildly on time scales that are much shorter than the dynamical timescale of largescale cool outflows. One should therefore use caution when comparing the properties of the AGN with the dynamical properties of cool outflows. We recommend using methods that are insensitive to short-term AGN variability when estimating the AGN luminosity. For instance, methods based on reprocessed radiation (e.g., mid-infrared emission) are preferable over methods based on the hard X-ray luminosity to mitigate the effects associated with short-term $\left(<10^{3}-10^{4} \mathrm{yr}\right)$ AGN variability.

\subsection{Driving Mechanisms}

The equation of motion of an outflowing shell fragment of mass $M_{s h}$ that subtends a solid angle $\Omega_{s h}$ is

$$
\frac{d}{d t}\left[M_{s h}(r) \dot{r}\right]=\Omega_{s h} r^{2}\left(P_{t h}+P_{C R}+P_{j e t}\right)+\left(\frac{\Omega_{s h}}{4 \pi}\right)\left(\frac{\tilde{\tau} L_{\mathrm{bol}}}{c}\right)-\frac{G M(r) M_{s h}(r)}{r^{2}},
$$

where $M(r)$ is the galaxy mass enclosed within a radius $r$. The terms on the right are the forces due to the thermal, cosmic ray, and jet ram pressures, the radiation pressure, and gravity, respectively. Below, we discuss each of the pressure terms separately. In reality, these pressure forces act together to drive the outflows. Examples of state-ofthe-art outflow simulations that incorporate many of these force terms are shown in Figures 2 and 3.

Fundamentally, cool outflows may be "energy-driven" or "momentum-driven", depending on whether radiative losses are negligible or not, respectively. In the first case, the outflow is adiabatic and the energy injected in the ambient gas is transformed into bulk motion at a rate $\dot{E}$, where $\dot{E} \propto \dot{M} v_{\text {out }}^{2}$. For starburst-driven winds, $\dot{E} \propto \dot{M}_{*}$ so the mass-loading factor $\eta \equiv \dot{M} / \dot{M}_{*} \propto v_{\text {out }}^{-2}$ (e.g., Murray et al. 2005). Energy-driven AGN winds where the energy injection rate scales with the black hole mass naturally lead to $M_{\mathrm{BH}} \propto \sigma_{*}^{5}$, where $\sigma_{*}$ is the stellar velocity dispersion of the host galaxy (e.g., Silk and Rees 1998; Murray et al. 2005, Sect. 2.2.1). The corresponding relations for momentum-driven outflows are $\dot{M} \propto \dot{E} v_{\text {out }}^{-1}, \eta \propto v_{\text {out }}^{-1}$, and $M_{\mathrm{BH}} \propto \sigma_{*}^{4}$ (e.g., Murray et al. 2005, Sect. 2.2.2). In that case, $v_{\text {out }}$ is also expected to linearly scale with the circular velocity $v_{\text {circ }}$ and, in starburst-driven outflows, $v_{\text {out }} \propto v_{\text {circ }} \dot{M}_{*}{ }^{0.25-0.50}$, where the exact dependence on the star formation rate depends on whether the area of the momentum injection zone scales linearly or not with the star formation rate. 


\subsubsection{Thermal Energy}

In this scenario, the shell fragment is pushed outward by ram pressure produced by the over-pressured hot gas in the central regions of the galaxy. The spherically symmetric case where radiative losses are negligible (i.e. energy-driven outflow) and the gravitational forces are also assumed to be negligible was developed analytically by Chevalier and Clegg (1985, CCC85) to explain the ionized wind of M 82 in the context of a supernova-driven outflow. The central pressure in the hot cavity in this case is $P_{0} / k \sim 3 \times 10^{5}\left(\dot{M}_{*} / M_{\odot} \mathrm{yr}^{-1}\right)\left(R_{*} / \mathrm{kpc}\right)^{-2} \mathrm{~K} \mathrm{~cm}^{-3}$, where $R_{*}$ is the radius of the injection zone (CC85; also Veilleux et al. 2005). The CC85 wind solution at large radii for a monoatomic gas with adiabatic index $\gamma=5 / 3$ has a constant asymptotic outflow velocity $v_{\text {hot }}^{\infty} \simeq \sqrt{5 k T_{\text {hot }} / \mu} \simeq 1500 \mathrm{~km} \mathrm{~s}^{-1}(\xi / \beta)^{1 / 2}$, a gas density $\propto r^{-2}$, and a gas pressure $\propto r^{-10 / 3}$, where $T_{\text {hot }} \simeq 1.5 \times 10^{7} \mathrm{~K}(\xi / \beta)$ is the temperature of the hot gas in the center, $\mu=1.36$ is the mean mass per particle, $\xi$ is the thermalization efficiency i.e. the fraction of the kinetic energy by the massive stars that is not lost to radiative cooling (Sec. 2.1.1), and $\beta$ is the ratio of the mass injection rate to the star formation rate. These results agree remarkably well with the X-ray observations of M82 with $\xi \sim 1$ and $\beta \sim 0.3$ (Strickland and Heckman 2009, Sec. 4.2.1). The momentum injection rate of this hot wind is $\dot{p}_{\text {hot }}=\dot{M} v_{X} \simeq(2 \dot{E} \dot{M})^{1 / 2} \simeq 5(\xi \beta)^{1 / 2} L / c$, where for the last approximate equality we used $L \simeq 10^{10}\left(\dot{M}_{*} / M_{\odot} \mathrm{yr}^{-1}\right) L_{\odot}$ and the relation between $\dot{E}_{\mathrm{SN}}$ and $\dot{M}_{*}$ for $\alpha_{S N}=0.01$ mentioned in Section 2.1.1. This expression for $\dot{p}_{\text {hot }}$ is similar to that of a radiation-pressure-driven wind in the optically thin limit (Sec. 2.2.2).

The first energy-driven wind model for AGN was developed by Silk and Rees (1998). In this model, the mechanical power of the wind is taken to be a constant fraction of the Eddington luminosity (thus $\epsilon_{f, k} \propto M_{\mathrm{BH}}$ in Eq. (5)) and results in a dependence on the host galaxy's velocity dispersion $\sigma_{*}$ of the form $M_{\mathrm{BH}} \propto \sigma_{*}^{5}$. This is somewhat steeper than the observed relation between the black hole mass and spheroid velocity dispersion (e.g., Gültekin et al. 2009; Kormendy and Ho 2013). A similar energy-conserving hot-bubble scenario has been proposed to explain the powerful molecular and atomic outflows seen in some ultraluminous infrared galaxies (Faucher-Giguère and Quataert 2012; Debuhr et al. 2012; Zubovas and King 2012, 2014; Costa et al. 2014; Nims et al. 2015). In this "blast-wave scenario", the violent interaction of a fast inner AGN wind with the ISM of the host results in shocked wind gas at a temperature of $2.0 \times 10^{10}\left(v_{\text {wind }} / 0.1 c\right)^{2} \mathrm{~K}$ that does not efficiently cool, but instead expands adiabatically as a hot bubble. The adiabatically expanding shocked wind sweeps up gas and drives an outer shock into the host ISM, raising the temperature of the ISM to $2.3 \times 10^{7}\left(v_{\text {shock }} / 1000 \mathrm{~km} \mathrm{~s}^{-1}\right)^{2} \mathrm{~K}$ (Nims et al. 2015). This outflowing gas cools radiatively, and most of it "freezes out" into clumps of cold molecular material (Fig. 3a; Richings and Faucher-Giguère 2018a,b). A variant on this scenario is that preexisting molecular clouds from the host ISM are entrained in the adiabatically expanding shocked wind, accelerated to the observed velocity without being destroyed by the many erosive forces and instabilities (the pros and cons of each of these scenarios are discussed in Section 2.3).

In both scenarios, we have by energy conservation $\frac{1}{2} \dot{M}_{\text {outflow }} v_{\text {outflow }}^{2}=\frac{1}{2} f \dot{M}_{\text {wind }} v_{\text {wind }}^{2}$, where the quantities with subscript "outflow" refer to the outer molecular/atomic out- 


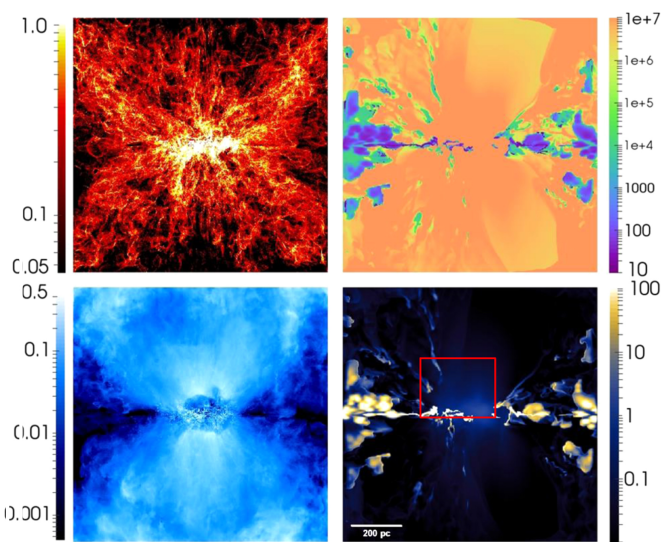

(a)

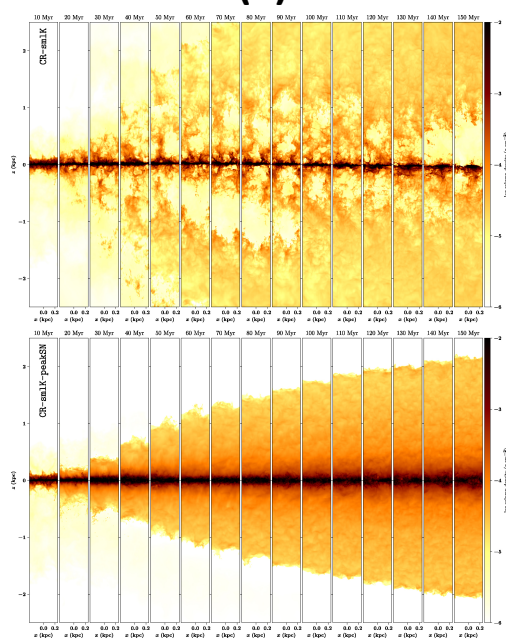

(c)

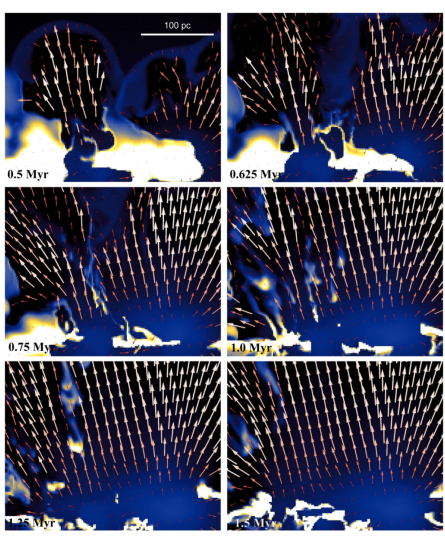

(b)

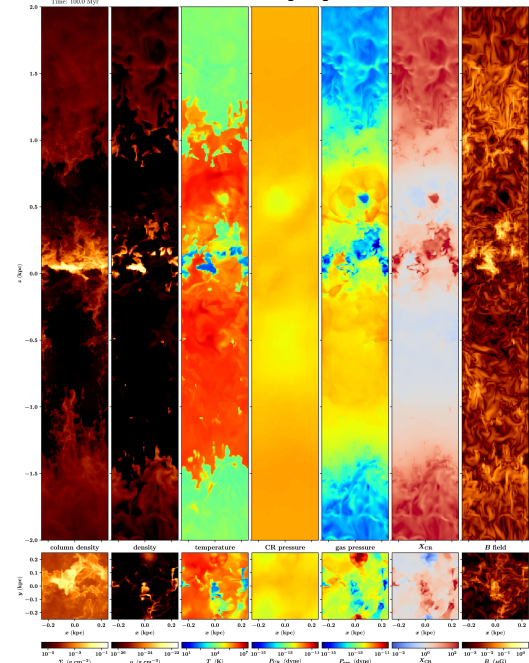

(d)

Fig. 2 Simulations of starburst-driven cool outflows: (a) Slice in $y z$ plane of a simulation at 1.5 Myr. Clockwise from top left: $\mathrm{H} \alpha$ emission ( $\left.\log \operatorname{erg~s}^{-1} \mathrm{~cm}^{-2}\right)$, temperature $(\mathrm{K})$, density $\left(\mathrm{cm}^{-3}\right)$, and soft X-ray emission ( $\log \mathrm{erg} \mathrm{s}^{-1} \mathrm{~cm}^{-2}$ ); red box indicates zoomed-in region in panel (b). (b) Close-up of the filamentforming region at six different epochs. (c) Time evolution of the edge-on column density for simulations dominated by SN thermal energy (top panel) and CR pressure (bottom panel) in steps of 10 Myr. (d) Vertical structure of the column density, density, temperature, CR pressure, thermal pressure, ratio of CR to thermal pressure, and magnetic field strength in a CR-dominated simulation at $t=100$ Myr. Images reproduced with permission from (a, b) Tanner et al. (2016), copyright by AAS; and (c, d) Girichidis et al. (2018), copyright by the authors.

flow, while those with subscript "wind" refer to the inner X-ray or UV AGN wind. The efficiency $f$ is defined as the fraction of the kinetic energy of the inner wind that goes into bulk motion of the swept-up molecular/atomic material. Rearranging the 
terms, we get

$$
\begin{aligned}
\dot{p}_{\text {outflow }} & =f\left(v_{\text {wind }} / v_{\text {outflow }}\right) \dot{p}_{\text {wind }} \\
& \simeq f\left(v_{\text {wind }} / v_{\text {outflow }}\right)\left(L_{\text {AGN }} / c\right),
\end{aligned}
$$

where the last equality is valid only if the inner wind is radiatively accelerated, i.e. $\dot{p}_{\text {wind }} \simeq L_{\mathrm{AGN}} / c$, which is often the case for X-ray or UV winds in AGN.

As long as the outflow remains small compared with the scale height or scale length of the ambient medium, the large-scale outflow will evolve through the same classic phases of evolution as those of individual SN remnants (Sec. 2.1.1). The energy-driven outflows discussed above will eventually transition into non-adiabatic pressure-driven snowplows when $T \lesssim 10^{6} \mathrm{~K}$ and radiative losses $\left(\propto n^{2}\right)$ are no longer negligible. Later on, once the thermal pressure of the hot gas equals that of the ambient pressure, the outflow will enter the momentum-conserving (momentum-driven) snowplow phase and rapidly slow down at it sweeps up more and more ISM gas. The terminal outflow velocity of a cloud accelerated outward by the ram pressure of a wind that carries momentum at a rate $\dot{p}$ into a solid angle $\Omega$ is (Heckman et al. 2011)

$$
v_{\text {ram }}=570 \dot{p}_{34}^{1 / 2}(\Omega / 4 \pi)^{-1 / 2} r_{0,100}^{-1 / 2} N_{21}^{-1 / 2} \mathrm{~km} \mathrm{~s}^{-1},
$$

where $\dot{p}_{34}$ is the rate of momentum carried by the wind normalized to $10^{34}$ dynes, $r_{0,100}$ is the initial launch radius of the cloud with respect to the central source normalized to $100 \mathrm{pc}$, and $N_{21}$ is the column density of the cloud normalized to $10^{21}$ $\mathrm{cm}^{-2}$. In cases where the injected energy and momentum from stellar and AGN processes are sufficient to drive an outflow on scales comparable to the host galaxy, the forces associated with pressure gradients in the underlying ambient ISM need to be taken into account and may help drive the outflow along the paths of least resistance (e.g., minor axis of a gaseous rotating disk).

\subsubsection{Radiation}

Direct UV radiation from massive young clusters absorbed by dusty gas can impart velocities to molecular clouds that are of order their escape velocities from the gravity of the cluster (Murray et al. 2011). Thus radiation can, in principle, drive cold galactic winds in starbursts with sufficiently high density of star formation activity if its pressure is efficiently coupled. The necessary condition is that the Eddington ratio of the radiation force to gravitational force is greater than unity. This is determined by the opacity per unit gas mass, $\kappa$, which is proportional to the dust-to-gas ratio, and the luminosity-to-mass ratio of the stellar population (Murray et al. 2010; Skinner and Ostriker 2015). In the single-scattering optically thin limit, the photon is absorbed by the gas or dust but is not re-emitted, so the momentum flux imparted onto the dust and gas is simply $L_{\text {absorbed }} / c \simeq\left(1-\mathrm{e}^{-\tau_{\mathrm{UV}}}\right) L_{\mathrm{BOL}} / c$ and the condition to drive an outflow is $L>L_{\mathrm{Edd}} \simeq 4 \pi G M c / \kappa_{\mathrm{UV}}$. This is somewhat relaxed in a turbulent medium, where under-dense lines of sight can be radiatively accelerated even when $L<L_{\text {Edd }}$ in the bulk of the material (Thompson and Krumholz 2016). Clouds that start as radiativelydriven experience gentler accelerations, and although they may later be advected in a hot flow and ultimately destroyed, they can potentially reach large distances and high 
velocities (Thompson et al. 2015). In this discussion, we have ignored the fact that heating of cold clouds irradiated by an energy source will create a pressure imbalance in the cloud and cause it to lose mass in the direction of the energy source. By the rocket effect, the cloud will experience an acceleration in the opposite direction (similar to what occurs in photoevaporative flows; e.g., Oort and Spitzer 1955; Bertoldi and McKee 1990). The results from recent numerical simulations of irradiated cold clouds embedded in flows of hot and fast material suggest that the impact of this extra kick in velocity on the accelerated clouds is not significant, except perhaps in the initial stage of acceleration (e.g., Brüggen and Scannapieco 2016).

Multiple scatterings, occurring when the optical depth to the re-radiated infrared $\tau_{I R}$ is large, could magnify the radiation force. A convenient expression that combines both single- and multiple-scattering processes is (Hopkins et al. 2014, 2018a)

$$
\dot{p}_{\text {rad }}=\tilde{\tau} L_{\text {bol }} / c \text { where } \tilde{\tau}=\left(1-e^{-\tau_{\text {single }}}\right)\left(1+\tau_{\text {eff, IR }}\right) .
$$

The value of $\tilde{\tau}$ ranges from $\sim \tau_{\text {single }}=\tau_{\mathrm{UV} / \text { optical }}<<1$ in the optically thin case to $\sim\left(1+\tau_{\text {eff,IR }}\right) \gtrsim 1$ in the infrared optically-thick limit. The effective infrared optical depth, $\tau_{\text {eff,IR }}=\kappa_{\text {eff,IR }} \Sigma_{\text {gas }}$, is the "boost factor"and is sometimes expressed as $\tau_{\mathrm{eff}, \mathrm{IR}}=\tau_{\mathrm{IR}} \beta_{\mathrm{IR}}$, the product of the integrated infrared optical depth, $\tau_{\mathrm{IR}}$, and a factor $\beta_{\mathrm{IR}}$ that includes all of the uncertainties in calculating the effective optical depth. To first order, the condition to drive a radiation-pressure wind under the optical-thick limit then becomes $L>L_{\text {Edd }} \simeq 4 \pi G M C / \kappa_{\text {eff,IR }}$ (note that the dust opacity $\kappa_{\text {eff, IR }} \propto v^{2}$ at the relevant IR wavelengths, so the radiation-gas coupling becomes less efficient as the radiation field diffuses outward of the dusty cocoon). In practice, the predicted importance of radiation pressure in driving cool outflows depends strongly on the method used to solve the frequency-dependent radiative transfer equation and derive this boost factor (see Krumholz et al. 2019, for a recent summary). Simulations based on the flux-limited diffusion (FLD) approximation suggest that such magnification is limited, as radiative Rayleigh-Taylor instabilities (RRTI) set in creating dense filaments of matter aligned with the radiation field that limit the momentum transfer and prevent gas ejection (Krumholz and Thompson 2013). Similarly, simulations using the M1 closure relation approximation find an anticorrelation between radiative flux and gas density that limits the radiation force magnification (Rosdahl and Teyssier 2015; Skinner and Ostriker 2015). Other simulations, using the more reliable variable Eddington tensor algorithm (e.g., Zhang and Davis 2017, Fig. 4c) or implicit Monte Carlo radiation transfer scheme (Tsang and Milosavljević 2015) are more successful at generating outflows after the onset of the Rayleigh-Taylor instabilities. Zhang and Davis (2017) have argued that $\beta_{\mathrm{IR}} \sim 0.5-0.9$, decreasing with increasing optical depth (see also Huang et al. 2019).

The above discussion equally applies to starburst- and AGN-dominated systems, although the star formation rate densities required to radiatively drive a wind may be very high (Crocker et al. 2018). Radiation pressure is likely the dominant driving force behind accretion-disk scale fast X-ray winds in AGN and UV broad absorption line (BAL) outflows in quasars, and may also play a crucial role in driving cool outflows. Momentum-driven outflows involving radiation pressure forces naturally produce $M_{\mathrm{BH}} \propto \sigma_{*}^{4}$ (e.g., Fabian 1999; King 2003; Murray et al. 2005; Ishibashi and Fabian 2018; Ishibashi et al. 2018b; Costa et al. 2018a,b). 


\subsubsection{Cosmic Rays}

Cosmic rays have the potential to efficiently accelerate cold gas, creating a neutral gas component in the galactic halo that could be an important mass reservoir in galaxies (Everett et al. 2008; Socrates et al. 2008; Uhlig et al. 2012). A gradient in cosmic ray pressure due to cosmic ray streaming and scattering in self-excited Alfvén waves can transport gas from the dense regions in the mid-plane to heights of kiloparsecs. The mass flow is overwhelmingly neutral and warm $\left(10^{4} \mathrm{~K}\right)$, but the velocities are slower than those obtained with supernova driving (Girichidis et al. 2016). The efficiency of the process is partially related to the global structure of the magnetic field, as cosmic rays need to stream away from the dense galactic regions along partially ordered magnetic field lines, but mass-loading factors of order unity appear feasible in a Milky Way-like galaxy (Ruszkowski et al. 2017). The so-called bottleneck effect, in which cosmic ray streaming through a cold cloud embedded in hot gas causes a cosmic ray pressure buildup in the neutral/molecular material and a resulting pressure gradient, can lead to cold cloud acceleration by cosmic rays (Wiener et al. 2017). This same effect can over-pressurize cold clouds and lead them to expand and have similar densities to the hot material (Werk et al. 2014).

The ability of CRs to drive galactic outflows is related to their overall scattering optical depth $\tau_{\mathrm{CR}} \sim R / \lambda$, where $R \sim \mathrm{kpc}$ is the distance the CRs have to travel to escape out of the galaxy and $\lambda$ is the uncertain mean free path of CRs between scattering. The effective momentum injection rate from CRs is then $\dot{p}_{\mathrm{CR}} \simeq \tau_{\mathrm{CR}} \dot{E}_{\mathrm{CR}} / c \simeq$ $10^{-3} \tau_{\mathrm{CR}}(L / c)$, where the last approximate inequality comes from the relations between $\dot{E}_{\mathrm{CR}}, \dot{M}_{*}$, and $L$ (Section 2.1.1 and Heckman and Thompson (2017)). The CR momentum injection rate may thus be comparable to the radiation pressure from massive stars if $\lambda \sim \mathrm{pc}$ and $\tau_{\mathrm{CR}} \sim 1000$. CR-driven winds are generally denser, colder, and smoother than the thermal supernova-driven outflows (Fig. 2c; e.g., Pakmor et al. 2016; Ruszkowski et al. 2017; Pfrommer et al. 2017; Gronke et al. 2018; Girichidis et al. 2018; Bustard et al. 2019; Vijayan et al. 2019).

\subsubsection{Radio Jets}

This section considers the ram-pressure term in Eq. (6) generated by collimated relativistic low-density jets from AGN. In this case, the jet injects an energy $E_{\text {jet }}=$ $\frac{1}{2} \rho_{j} 2 \pi r_{\text {in }}^{2}\left(1-\cos \theta_{J}\right) v_{J}^{3} t_{J}$, where $\rho_{J}, v_{J}, \theta_{J}$, and $t_{J}$ are the jet mass density, velocity, half-opening angle, and duration, respectively, and $r_{\text {in }}$ is the inner radius at the base of the jet where the jet energy is injected into the medium (Bambic and Reynolds 2019). The impact of this jet on the ambient medium not only depends on $E_{\text {jet }}$, but also on the level of interaction of the jet with the medium. For instance, a powerful, highly collimated jet will simply "drill” through the host galaxy's ISM without imparting much energy and momentum to it (e.g., Scheuer 1974; Mukherjee et al. 2016). The other extreme case where all of the jet kinetic energy is deposited on small scale to create a hot over-pressured bubble has already been discussed in Section 2.1.1 in the context of thermally-driven outflows in starbursts (see also Mukherjee et al. 2016). Fine-tuning of the jet power, kinetic feedback efficiency, $\epsilon_{f, k}$, and ISM cloud structure is therefore needed for efficient energy and momentum transfer to disperse a 
significant fraction of the gas in galaxies (Wagner et al. 2012; Mukherjee et al. 2016, 2018a,b). Typical jet powers needed to create a jet-driven energy bubble with the right properties are $\dot{E}_{\text {jet }} / L_{\text {Edd }} \gtrsim 10^{-4}$. Material accelerated at the base of the jets may reach sub-relativistic velocities that are consistent with ultra-fast outflows detected in radio-loud AGN (Wagner et al. 2013). While these radio jets launch strong outflows, virtually none of the entrained mass reaches escape velocity so it rains back onto the galaxy as a galactic fountain. In the special case of jets directed through the plane of a galaxy disk, the jet may stir the kinematics of the multi-phase ISM and affect the efficiency of star formation (Fig. 3b, Mukherjee et al. 2018b). We discuss this issue in more details in Sect. 4.5, 3.4.6 and 5.2 below.

\subsection{Physical Origin of the Outflowing Cool Gas}

Mass loading of outflows with cool gas can arise from two fundamental causes: mechanical drag leading to the entrainment of cool gas in a hot, fast-moving fluid, and direct acceleration of the cool gas through radiation or cosmic ray pressure (e.g., Kim and Ostriker 2018). A third possibility is to create the cool gas starting from the hot wind material, through cooling and condensation. Part of the complexity of understanding the driving of galactic outflows is due to the fact that several of these processes may be taking place simultaneously. Hot outflows from the blowout of over-pressured regions surrounding a starburst will be concomitant with large radiation fields and the associated radiation pressure, for example, making it difficult (and to some extent artificial) to separate them. It has been suggested that, in order to drive cool outflows efficiently in the context of galaxy evolution, several of these mechanisms need to operate simultaneously (Hopkins et al. 2012). Below we discuss in more detail entrainment and in situ formation. Direct acceleration by radiation and CR pressure has already been discussed in Sections 2.2.2 and 2.2.3, respectively, so it is not repeated here.

\subsubsection{Cool Gas Entrainment}

The acceleration of cold clouds by a warm, fast wind has proven a difficult problem to solve. The density contrast between the cool material and the hot supersonic wind is $\chi \simeq 10^{3}-10^{4}$ (e.g., Scannapieco and Brüggen 2015), which already suggests that imparting momentum to a dense "cloudlet" is going to require a substantial flow of the lighter outflow warm fluid. In the standard acceleration calculation, a dense cloudlet is placed in the fast hot flow. When the flow encounters the cloud, it develops a shock that compresses it and starts to ablate and shred it on a "cloud crushing" timescale, $t_{c c}$, equal to the ratio of the ratio of the time for the hot flow to travel the radius of the cloud multiplied by $\chi^{1 / 2}$ (Klein et al. 1994). Accelerating the cloud to the speed of the wind requires that the mass in the flow that impacts the cloud is similar to the mass of the cloud itself. This in turn requires a length of time that is $t_{a c c} \sim \chi^{1 / 2} t_{c c} \gg t_{c c}$. Therefore, in the absence of a stabilization mechanism, it would appear that clouds are destroyed through Rayleigh-Taylor fragmentation and shear-driven Kelvin-Helmholtz instabilities induced by the flow faster that they can 


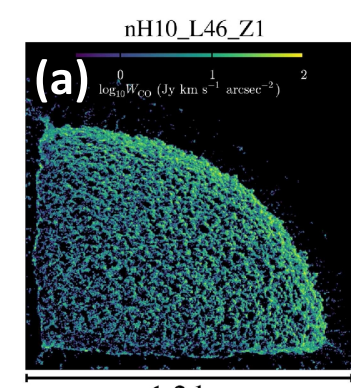

$1.2 \mathrm{kpc}$

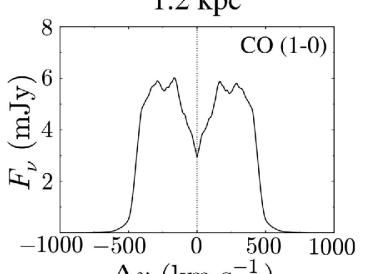

$\Delta v\left(\mathrm{~km} \mathrm{~s}^{-1}\right)$

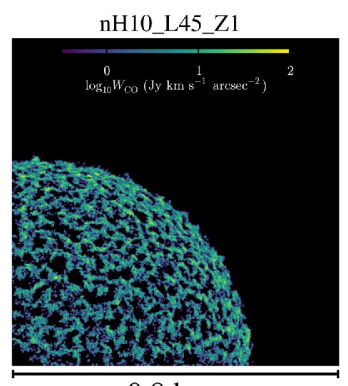

$0.8 \mathrm{kpc}$

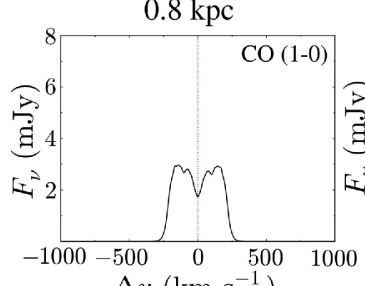

$\Delta v\left(\mathrm{~km} \mathrm{~s}^{-1}\right)$
nH10_L46_Z0.1

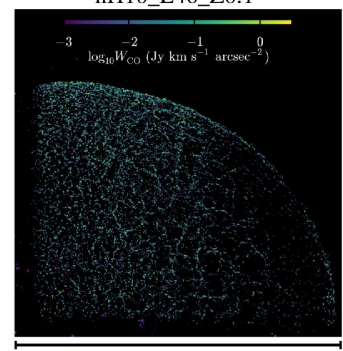

$1.2 \mathrm{kpc}$

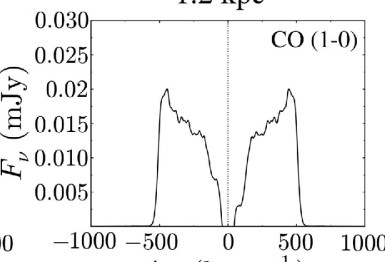

$\Delta v\left(\mathrm{~km} \mathrm{~s}^{-1}\right)$

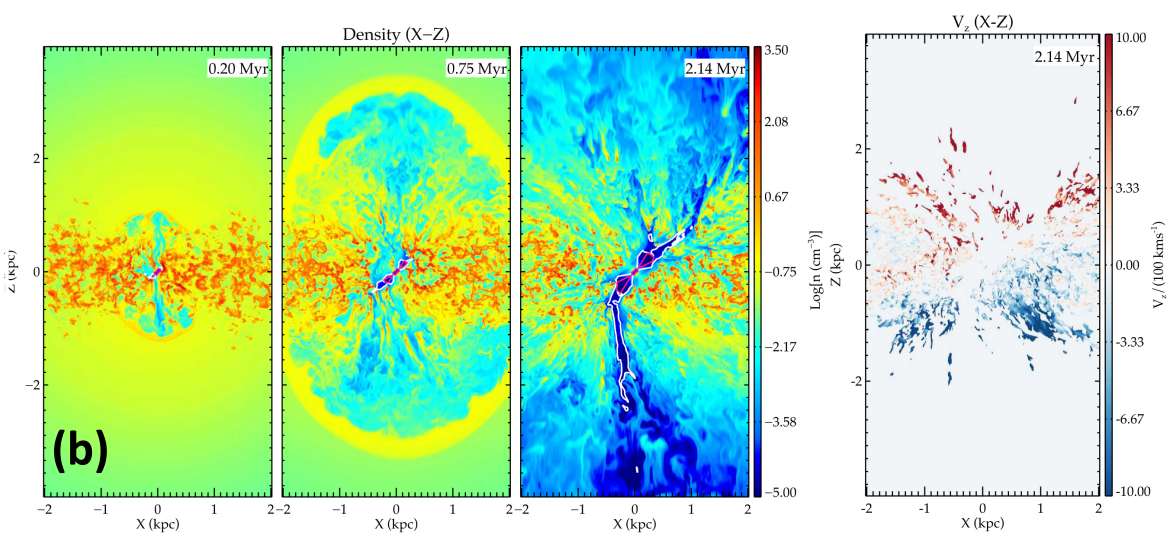

Fig. 3 Simulations of AGN-driven cool outflows: (a) Velocity-integrated maps of CO (1-0) emission (top row) and $\mathrm{CO}(1-0)$ spectra (bottom row) for three different simulations. (b) Left three panels: timeevolution $(0.20,0.75$, and $2.14 \mathrm{Myr})$ of the density in a jetted outflow simulation, where the jet is tilted by $45^{\circ}$ from the plane of the disk. The right panel shows the vertical velocity field in units of $100 \mathrm{~km} \mathrm{~s}^{-1}$ at 2.14 Myr. Images reproduced with permission from (a) Richings and Faucher-Giguère (2018b) and (b) Mukherjee et al. (2018b), copyright by the authors.

be accelerated by it, a result also obtained by detailed hydrodynamic simulations (Cooper et al. 2009; Scannapieco and Brüggen 2015; Schneider and Robertson 2017). Clouds with realistic density distributions are destroyed faster than spherical clouds of uniform density (Schneider and Robertson 2015). The material from the destroyed cloud joins the flow, mass-loading the hot phase.

Possible stabilization mechanisms for cold material advected in a fast flow are radiative cooling and magnetic fields. As the cloud experiences the initial crushing shock its density increases. If the material can efficiently cool radiatively, the denser 
clumps formed will likely survive for a longer period of time (Cooper et al. 2009). In fact, if the cooling is fast enough compared to the cloud crushing time, a cloudlet may grow in mass by acting as a condensation seed that accretes newly cooled gas (Marinacci et al. 2010; Armillotta et al. 2016, 2017; Gronke and Oh 2018, 2019). In supersonic flows Kelvin-Helmholtz instabilities are suppressed, slowing down cloud disruption, and as the cloud is compressed it develops a pressure gradient along the flow direction that causes a filamentary structure to develop. The lower cross section to the flow means the drag forces are reduced, resulting in a slower acceleration despite the increased lifetimes (Scannapieco and Brüggen 2015). Clouds with efficient thermal conduction experience ablation of their outer layers, in contact with the hot flow, which results in an additional acceleration due to the rocket effect but also hastens their destruction (Marcolini et al. 2005). The lifetime of the cloud in these circumstances depends on the balance between radiation, conduction, and the disrupting instabilities (Orlando et al. 2005), but it appears that on balance longer lifetimes do not translate into reaching faster flow velocities before destruction (Brüggen and Scannapieco 2016). Magnetic fields can suppress conduction (Orlando et al. 2008), and tangled magnetic fields threading a cloud may help stabilize it against disruption (McCourt et al. 2015). In strongly magnetized winds, simulations suggest that magnetized clouds experience additional drag forces that result in much faster accelerations (McCourt et al. 2015; Banda-Barragán et al. 2016, 2018, 2019; Grønnow et al. 2018). There may be some recent observational support for this magnetized outflow scenario in one object (Leaman et al. 2019). The effects of the different cloud destruction mechanisms are a function of parameters such as the cloud column density and the temperature of the medium, with different physics dominating in different regions of parameter space (Li et al. 2019).

\subsubsection{In-Situ Formation from the Hot Wind Material}

It is possible for a cold phase to form in situ via thermal instabilities and condensation from hot gas with a cooling time shorter than its dynamical time (Efstathiou 2000; Silich et al. 2003). Because adiabatic cooling due to expansion acts to lower the gas temperature into a regime where runaway radiative cooling takes over, this appears to be a general expectation. The efficiency of this process is strongly dependent on the mass-loading of the hot phase, thus increased mass-loading due to destruction of entrained cold clouds in the inner regions of the flow may play an important role in causing thermal instabilities to develop (Thompson et al. 2016). Also, systems with larger surface densities of star formation rate are expected to have shorter cooling times. In strongly mass-loaded systems with high star-formation rates, which may correspond to outflows from ULIRGs, the result from the cooling instability may be material that has enough density to turn molecular and enough column density to selfshield from the dissociating metagalactic radiation field (Thompson et al. 2016). In this picture X-ray emission arises from recombination, as the outflow progressively cools down, which may explain the strong spatial coincidence between $\mathrm{H} \alpha$ and soft $\mathrm{X}$-ray emission observed in galaxy winds. Because of the expected strong correlations between mass loading, velocity, and temperature, this scenario predicts a correlation between the observed wind velocities and the local escape velocity, as well as faster 


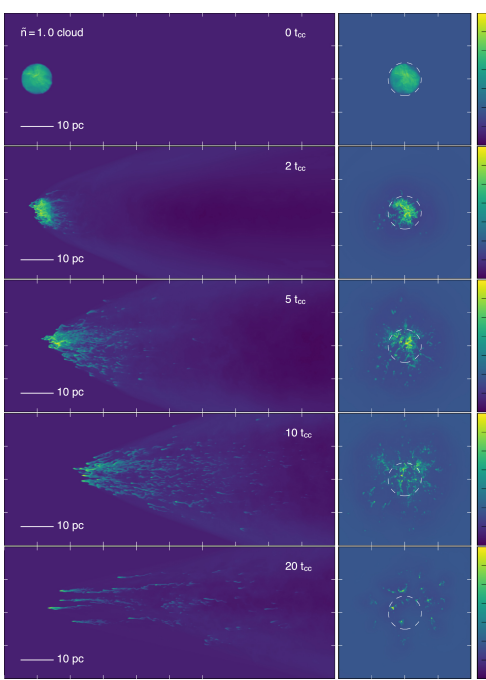

(a)

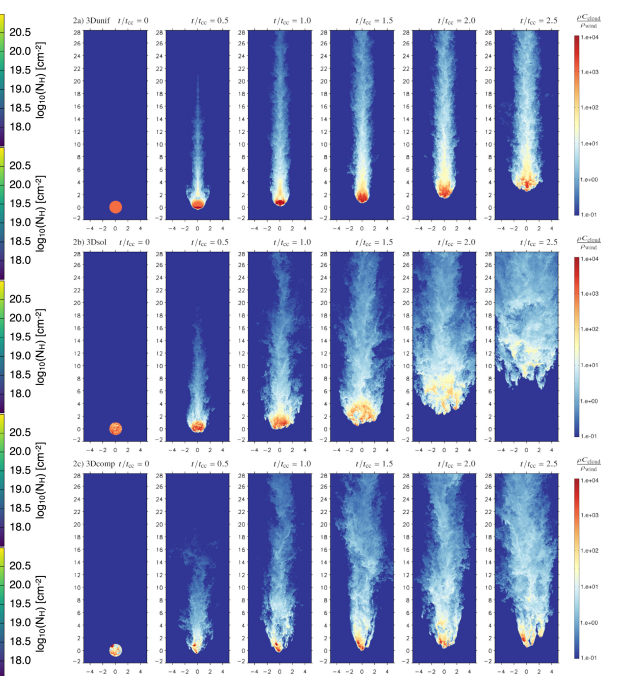

(b)
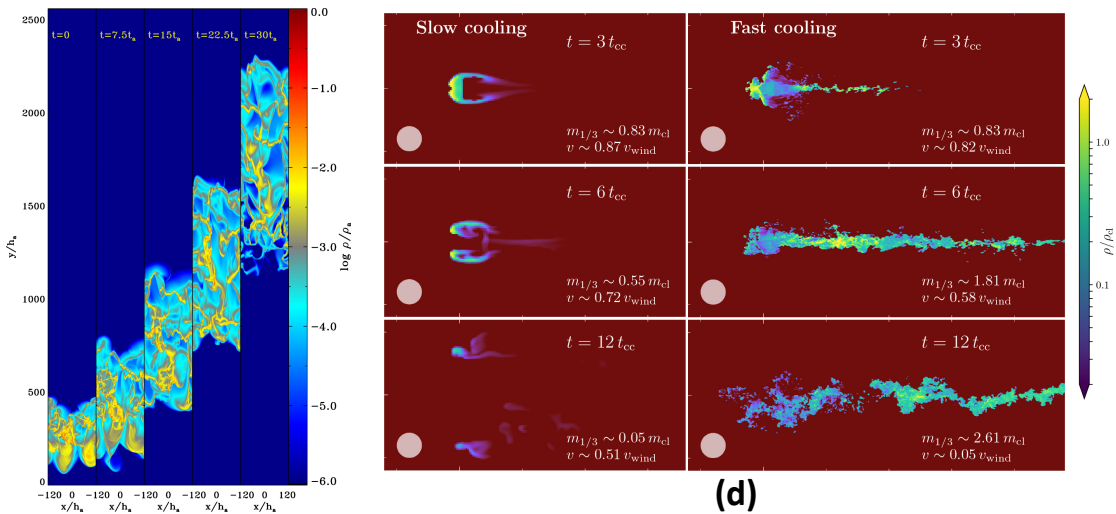

(d)

(c)

Fig. 4 Simulations of accelerated cool clouds: (a) Time series evolution of a turbulent cloud embedded in a wind. The panels on the left show the surface density projected along the y-axis, with the wind entering the box on the left, while the right column shows the same quantity in the direction of the wind velocity. (b) Same as (a) but for different internal density distributions. The wind enters from the bottom in this case. (c) Density distribution at five different epochs, from two-dimensional radiation hydrodynamical simulations of dusty winds. (d) Density distributions at three different times when cooling is slow (left panels) and fast (right panels). Images reproduced with permission from (a) Schneider and Robertson (2017), (c) Zhang and Davis (2017), copyright by AAS; and (b) Banda-Barragán et al. (2019), (d) Gronke and Oh (2018), copyright by the authors.

velocities for the lower column densities (Martin 2005; Rupke et al. 2005c). The ultimate fate of the wind would be to populate halos with cool gas, as the expanding wind cools adiabatically first to a point where runaway cooling takes over, or shocks the ambient circumgalactic medium which then cools in less than a Hubble time (Thompson et al. 2016). 
Can cooling and condensation explain the observations of molecular material in winds? For the material to become molecular, hydrogen atoms need to combine to produce molecular hydrogen. Because of the symmetry of the system and the lack of a permanent dipole, gas phase reactions between neutral atoms for formation of $\mathrm{H}_{2}$ proceed extremely slowly (Gould and Salpeter 1963). The dominant gas channel for $\mathrm{H}_{2}$ formation in the gas phase starts with the formation of a $\mathrm{H}^{-}$ion through radiative association, followed by formation of $\mathrm{H}_{2}$ via associative detachment. But this process is also slow, and likely dominant only in the very early universe (Glover et al. 2006). To explain its abundance in the dense ISM, it has been long accepted that $\mathrm{H}_{2}$ formation is catalyzed by dust grains, where the dust surface provides a substrate for $\mathrm{H}$ atoms to find each other efficiently and combine (Gould and Salpeter 1963; Hollenbach and Salpeter 1971). If the dust grains immersed in low density, high temperature gas are warm, this may impact their ability to form molecular hydrogen (note that the equilibrium temperature of the grains is determined by the radiation field at these densities). The precise mechanism through which the dust catalysis of $\mathrm{H}_{2}$ formation occurs, and hence its temperature dependence, are matters of debate, and hence it is unclear how efficiently $\mathrm{H}_{2}$ formation would proceed on warm dust-grain surfaces (Le Bourlot et al. 2012). Moreover, dust grains immersed in hot gas experience destructive sputtering due to grain charging and gas-grain collisions. Dust grains of size $\sim 0.1 \mu \mathrm{m}$ immersed in a $T>10^{6} \mathrm{~K}$ hot plasma of density $\sim 10^{-2} \mathrm{~cm}^{-3}$ have a lifetime of only a few $10^{6}$ years (Draine and Salpeter 1979a, see eq. 61 in Sec. 3.5.6 below). This implies that even when neglecting the effects of shocks (which are also very destructive, Jones et al. 1994; Slavin et al. 2015), once grains are immersed in the hot phase they only last for a few million years before being destroyed. Taken together, this suggests that reforming a molecular phase from hot material through cooling and condensation could be very difficult.

Independently, it seems clear that many of the observations of molecular gas in winds cannot be explained by material that is condensed from the hot phase. The mm-wave observation of complex molecules $\left(\mathrm{HCN}, \mathrm{HNC}, \mathrm{HCO}^{+}\right)$in some of the molecular outflows strongly suggests that the gas started as molecular, dense, and chemically rich (Aalto et al. 2012; Walter et al. 2017). Some of these molecular transitions appear enhanced in outflows, but this is likely to be due to either shock chemistry or excitation effects, not incorporation of material condensed from the hot phase (Aalto et al. 2012). Some observations show that the dynamical age of the outflowing features that contain these molecules is short, likely too short for the molecules to have formed in the flow. Time-dependent calculations of chemistry and molecular cloud formation in colliding flows find that, even in relatively dense and benign conditions in the neutral galactic ISM, it takes a few Myr for the material to become predominantly molecular, and several Myr for it to emit brightly in CO (Clark et al. 2012). By contrast, the dynamical time scale of some CO-emitting features in outflows is $\sim 1 \mathrm{Myr}$ (for example in NGC 253, Walter et al. 2017), strongly pointing to an entrainment origin for the molecular gas. 
2.4 Minimum Requirement for the Outflowing Material to Escape the Host Galaxy

An important question is whether the outflowing material escapes the host galaxy altogether or remains bound to it and thus is deposited in the CGM. A necessary condition to escape the gravitational potential of a galaxy is for the material to have a radial outflow velocity that exceeds the local escape velocity (it is not a sufficient condition since drag forces may be important). Assuming a spherically symmetric galaxy with gravitational potential $\Phi(r)$, the local escape velocity is given by $v_{\mathrm{esc}}^{2}(r)=2[\Phi(\infty)-\Phi(r)]$. For a singular isothermal sphere, where the mass $M(<r) \mathrm{di}-$ verges at large $r$ but the circular velocity $v_{\text {circ }}$ is a constant, $v_{\text {esc }}^{2}(r)=2 v_{\text {circ }}^{2} \ln \left(r_{\text {halo }} / r\right)$, so it slowly diverges for large $r_{\text {halo }} / r$ (Binney and Tremaine 2008). To avoid this divergence, one often truncates the singular isothermal sphere at a radius $r_{\text {halo }}=r_{\max }$. In that case, $v_{\mathrm{esc}}^{2}(r)=2\left[\Phi(\infty)-\Phi\left(r_{\max }\right)+\Phi\left(r_{\max }\right)-\Phi(r)\right]=2 v_{\text {circ }}^{2}\left[1+\ln \left(r_{\max } / r\right)\right]$, so

$$
v_{\mathrm{esc}}(r)=v_{\mathrm{circ}} \sqrt{2\left[1+\ln \left(\frac{r_{\mathrm{max}}}{r}\right)\right]} .
$$

In more realistic galaxies where the gas is supported by a combination of rotational and random motions, the circular velocity may be replaced by $v_{\text {circ }}=\sqrt{2} S$, where the kinematic parameter $S$ is defined as $S^{2} \equiv 0.5 v_{\text {rot }}^{2}+\sigma_{\text {gas }}^{2}\left(v_{\text {rot }}\right.$ and $\sigma_{\text {gas }}$ are the observed rotation velocity and velocity dispersion of the ISM in the host galaxy) and scales with the stellar mass following $\log S=0.29 \log M_{*}-0.93$, where $S$ is in $\mathrm{km}$ $\mathrm{s}^{-1}$ and $M_{*}$ is in $M_{\odot}$ (Weiner et al. 2006; Kassin et al. 2007), in low- $z$ star-forming galaxies (Heckman et al. 2015; Simons et al. 2015). The value of $v_{\mathrm{esc}}$ is not sensitive to $r_{\max } / r$ (e.g., for $r_{\max } / r=10-100, v_{\mathrm{esc}} / v_{\text {circ }}=2.6-3.3$ ). A rather conservative simplification when studying galaxy-scale outflows is to choose $v_{\text {esc }}\left(r_{\text {outflow }}\right) \simeq 3 v_{\text {circ }}$, which implicitly assumes $r_{\text {max }} / r_{\text {outflow }} \simeq 33$. When studying CGM-scale flows, the radius $r_{\text {max }}$ may be substituted with the virial radius $r_{\text {vir }} \simeq v_{\text {circ }} / 10 H(z)$ (in Mpc), where $H(z)$ is the Hubble parameter at redshift $z$ (e.g., Schroetter et al. 2016). In that case, $v_{\mathrm{esc}}\left(r_{\mathrm{CGM}}\right) \simeq 2 v_{\text {circ }}$ generally is a reasonable approximation $\left(r_{\mathrm{vir}} / r_{\mathrm{CGM}} \simeq e\right)$. The issue of the fate of the cool outflowing material is discussed in Sections 5.6 and 6.4.

\section{Observational Techniques and their Limitations}

This section is a critical review of the methods used to detect and characterize the properties of cool outflows in galaxies, near and far. First, we briefly discuss the most commonly used criteria to distinguish the outflowing gas from the quiescent material in the host galaxy, and describe the basic formulae used to derive the key dynamical quantities of the outflow. The remainder of this section describes from a practical point of view the various techniques and diagnostics that have been used to detect and characterize the outflowing material, starting with the neutral-atomic gas, followed by the molecular gas, and concluding with the dust in these outflows. 


\subsection{Kinematic Definition of an Outflow}

All of the properties of the outflows discussed in this review rely on a robust decomposition of the outflowing material from the dominant non-outflowing material. This is a non-trivial task that adds uncertainties to the outflow properties.

\subsubsection{Spatially Unresolved Outflows}

When the outflow is spatially unresolved, one has to rely on the detection of faint wings in the velocity profile of the spatially integrated absorption and/or emission line to determine the portion of the gas that is outflowing out of the galaxy. Emission line features have the disadvantage of being ambiguous in terms of flow direction, when the line-emitting gas is optically thin and unaffected by dust or other absorbing material within the outflow structure. Velocity thresholds have traditionally been used to identify the outflowing gas, but these thresholds vary wildly in the literature. In principle, these kinematic thresholds should be adjusted on an object-by-object basis to account for the range of velocities associated with gravitational motions (rotation, random motion, inflow), based on the mass of the host galaxy. Parametric decomposition of the observed profiles into a "narrow" and "broad" velocity components is often carried out to quantify the fraction of the emission / absorption that lies in the wing(s) of the profiles. The dominant narrow component, responsible for the core of the line profile, is associated with gas in the gravitational potential of the host galaxy while the broad component is associated in part with the outflow. The width of the narrow component is a proxy for the mass of the galaxy and therefore can be used to define the kinematic threshold above which the outflowing material becomes significant in the broad component (e.g., Lutz et al. 2019)

The use of the formulae in Section 3.2 requires a characteristic outflow velocity. This velocity may be derived from the above parametric decomposition procedure or using non-parametric methods such as interpercentile range measurements (e.g., Whittle 1985; Veilleux 1991). Typically, one selects $v_{50}$ or $v_{84}$. The maximum outflow velocity (e.g., 98-percentile velocity, $v_{98}$, of the integrated or broad-component profile) should not be used in these formulae since (1) it overestimates the characteristic velocity and thus the energetics of the outflow, and (2) it is much more sensitive to noise than other more appropriate measurements of the maximum outflow velocity such as $\left|v_{84}\right|$ or $\left|v_{50}\right|+2 \sigma$, where $\sigma$ is the velocity dispersion of the broad component (note that $\left|v_{98}\right|=\left|v_{50}\right|+2 \sigma$ for a Gaussian velocity distribution; e.g., Rupke and Veilleux 2013b).

\subsubsection{Spatially Resolved Outflows}

The identification of the outflowing gas in cases where the outflow is spatially resolved usually relies on comparisons between the observed velocity field and line width distribution and the predictions from that of a differentially rotating disk of material with some level of random motion normal to the disk, taking into account the finite spectral resolution of the instrument as well as the effects of beam smearing of the velocity field associated with the finite width of the instrumental beam size. Beam 
smearing will effectively flatten velocity gradients and broaden the widths of the observed line profiles where the gradients are steeper (e.g., ${ }^{3 \mathrm{D}} \mathrm{BAROLO}$; Di Teodoro and Fraternali 2015). This method works well when the outflow is fast and powerful but fails when the signatures of the outflow in the line profiles are more subtle. The next level of analysis consists in trying to capture these more subtle signs of the outflow in the line profiles (shoulders, wings) by parametrizing the line profiles at each position in the outflow and galaxy as a sum of 2-3 components and assign the component that shows signatures of rotation (e.g., clear velocity gradient in the same direction as the stars) to the gravitationally dominated component in the host galaxy and the other(s) to the outflow (e.g., Rupke and Veilleux 2013b). The definitions discussed in Sec. 3.1.1 for the characteristic velocity and flux assigned to the outflow material can then be applied for the individual profiles at each position. Constraints or initial guesses on the parameters of the individual components based on the results of fits in the neighboring pixels may be used to guard against unphysical discontinuities on sub-beam scale. An iterative method may be used to remove contamination from a bright central point source (e.g., quasar; Rupke et al. 2017).

The above method will fail if the line-emitting material is not well resolved by the beam. Assuming the source is strongly detected, one solution is to look for the residuals in the integrated profiles after fitting and removing a Gaussian source model at each velocity channel (e.g., Veilleux et al. 2017). First, a two-dimensional Gaussian is fit to the image for each channel in the region where a source is detected. These results are then used to make a smooth source model with linearly changing position as a function of velocity (to account for a possible velocity gradient), Gaussianchanging intensity, but constant size and orientation. This smooth source model is then removed from each velocity slice to arrive at a "residuals" cube. The high $\mathrm{S} / \mathrm{N}$ of the detection allows to centroid the source in each channel with very good subbeam precision. The "high-velocity" emission, which cannot be accounted for by the gas in pure rotation, is identified as the outflowing material. In cases where the data cubes are based on interferometric data, the results from the analysis on the imaging data may also be checked independently by deriving the sizes and fluxes of the wing emission from a fit of the data directly in the $u v$ plane (e.g., using CASA $u v$-Plane Model Fitting routines uvmodelfit and uvmultifit from the library of Martí-Vidal et al. 2014).

\subsection{Derivation of Outflow Energetics: Basic Formulae}

In cases where the outflow is not spatially resolved, one has to assume an outflow model. The simple case of an optically thin, spherically symmetric mass-conserving free wind, with an instantaneous mass outflow rate and velocity that are independent of radius within the wind and zero outside, is discussed in Rupke et al. (2005c). The mass outflow rate at a radius $r$ within the wind is given by

$$
\dot{M}=\Omega \mu m_{p} n(r) v r^{2},
$$

where $m_{p}$ is the proton mass, $\mu \simeq 1.36$ is the mass per $\mathrm{H}$ nucleus taking into account the relative He abundance, $v$ is the outflow velocity, and $\Omega$ is the solid angle subtended 
by the wind as seen from its origin (i.e. the wind's global covering factor). This last term includes a large-scale covering factor (related to the wind's opening angle), given by $C_{\Omega}$, and a small-scale covering factor (related to the wind's clumpiness), $C_{f}$. Thus, $\Omega / 4 \pi=C_{\Omega} C_{f}$ (Rupke et al. 2005b). The hydrogen number density $n(r)$ is related to the measured hydrogen column density $N=\int_{r_{1}}^{r_{2}} n(r) d r$ for a wind extending from inner radius $r_{1}$ to outer radius $r_{2}$. Assuming a density profile $n(r) \propto r^{-2}$ (e.g., an isothermal sphere), the instantaneous (local) mass outflow rate across any radius $r$ within the wind is

$$
\dot{M}_{\text {thick }}^{\text {inst }}=\Omega \mu m_{p} N v \frac{r_{1} r_{2}}{\Delta r},
$$

where $\Delta r=r_{2}-r_{1}$, and the corresponding mass of the wind is

$$
M_{\text {thick }}=\Omega \mu m_{p} N r_{1} r_{2} \text {. }
$$

The mass outflow rate averaged over the wind lifetime $\sim r_{2} / v$ is

$$
\dot{M}_{\text {thick }}^{a v g}=\Omega \mu m_{p} N v r_{1},
$$

If the wind is a thin shell of radius $r \sim r_{1} \sim r_{2}$, the corresponding equations are

$$
\begin{aligned}
& \dot{M}_{\text {thin }}^{\text {inst }}=\Omega \mu m_{p} N v \frac{r^{2}}{\Delta r}, \\
& \dot{M}_{\text {thin }}^{\text {avg }}=\Omega \mu m_{p} N v r, \\
& M_{\text {thin }}=\Omega \mu m_{p} N r^{2} .
\end{aligned}
$$

Note that, in both the thick- and thin-shell cases, the time-averaged mass outflow rates are a factor of $\Delta r / r$ lower than the local instantaneous rates.

The momenta and kinetic energies and their outflow rates are

$$
\begin{aligned}
& p=M v, \\
& \dot{p}=\dot{M} v, \\
& E=M\left(v^{2} / 2+3 \sigma^{2} / 2\right), \\
& \dot{E}=\dot{M}\left(v^{2} / 2+3 \sigma^{2} / 2\right),
\end{aligned}
$$

where the energy includes both the "bulk" kinetic energy due to the outflowing gas and "turbulent" kinetic energy (where we assume the same velocity dispersion $\sigma$ in each dimension). ${ }^{1}$

Recall that $n(r) \propto r^{-2}$ was assumed. All of the above quantities need to be multiplied by a factor of 3 if the spherical (or multiconical) volume is instead filled with uniform density. Note, however, that the quick drop-off in the radial intensity profile generally seen in outflows seems inconsistent with this picture (Fig. 5). Thus we favor the above equations for the calculations of the energetics.

\footnotetext{
1 Note that there is a typographical error in the numerical formulae of Rupke et al. (2005c): in their equations 13-18, the normalization factor of the column density $N$ should be $10^{20} \mathrm{~cm}^{-2}$ rather than $10^{21}$ $\mathrm{cm}^{-2}$. The outflow energetics published in Rupke et al. (2005c) are based on the correct formulae and not affected by this error.
} 

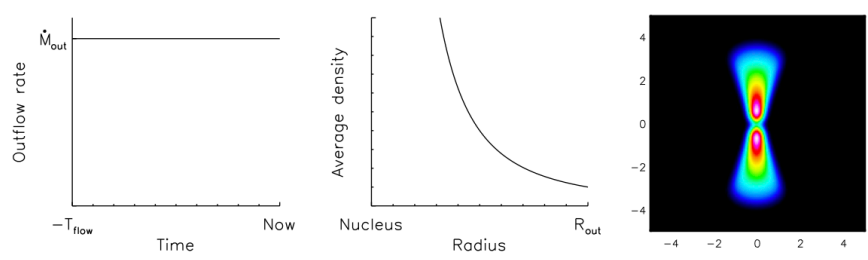

$$
\dot{M}_{\text {out }}=v_{\text {out }} \frac{M_{\text {out }}}{R_{\text {out }}}
$$
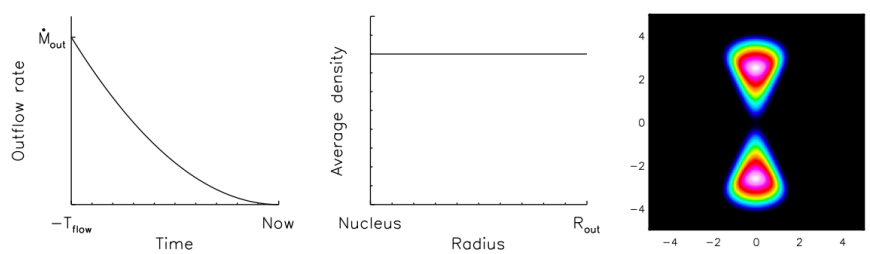

$$
\dot{M}_{\text {out }}=3 v_{\text {out }} \frac{M_{\text {out }}}{R_{\text {out }}}
$$
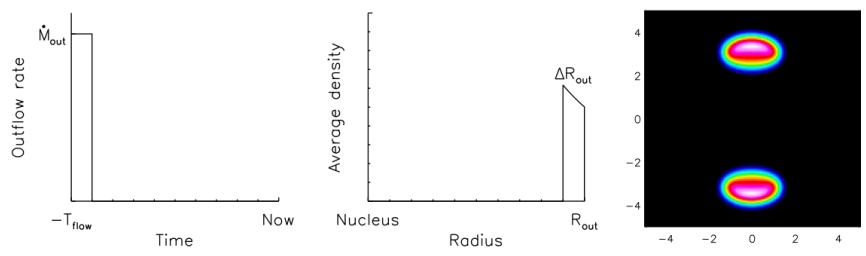

$\dot{M}_{\text {out }}=v_{\text {out }} \frac{M_{\text {out }}}{\Delta R_{\text {out }}}$

Fig. 5 Three commonly adopted outflow histories and their effects on the radial density profile and conversion from outflow mass to outflow mass rate. From left to right, the panels show the outflow history, the average radial density profile (local cloud conditions may differ from this average), the simulated moment 0 map (for a modest $20^{\circ}$ half opening angle flow seen with flow axis inclined by $60^{\circ}$ with respect to the line of sight), and the equation for conversion from outflow mass to outflow mass rate. From top to bottom, the three cases are constant outflow history, constant average volume density in cone (requiring a decaying outflow history), and thin outflowing shell. Images reproduced with permission from Lutz et al. (2019), copyright by the authors.

In cases where the wind is spatially resolved, the integrated mass of outflowing material is simply $M=\mu m_{p} \int N d A$, where the integral of the two-dimensional hydrogen column density distribution of the outflowing material is carried out over the projected area of the outflow. If the observed outflow velocity varies with position, then one needs to modify the above equations for the outflow mass, momentum, and energy rates by letting each position on the sky (corresponding to a unique angular coordinate with respect to the wind's center) have its own velocity, mass, momentum, and energy on the thin shell (see Shih and Rupke 2010). The outflow rates are derived by dividing these quantities by the dynamical time $r / v$ at each position in the wind. Note that all of the above formulae are expressed in terms of the column density, and are in this form more appropriate for absorbing material. In cases of line-emitting material, then one needs to compute the gas mass at each location on the sky.

Projection effects add uncertainties to the results of these computations. In nearby systems where the outflows are well resolved, attempts have been made to account for these projection effects by fitting the observed velocity field with the predictions from simple three-dimensional kinematic models (e.g., bipolar bubbles, biconical outflows, filled-in geometries instead of shells; e.g., Veilleux et al. 1994; Rupke and Veilleux 2013b). This often cannot be done at higher redshifts. 
3.3 Diagnostic Tools of the Neutral-Atomic Gas Phase

A broad diversity of techniques and diagnostic tools have been used to study the neutral atomic component of galactic outflows (Fig. 6). These tools and techniques can be divided into three broad categories based on the use of the strongest transitions from: (1) hydrogen itself, (2) the most abundant neutral chemical elements, or (3) the most abundant singly-ionized chemical elements with first ionization potential below $13.6 \mathrm{eV}$. Below we discuss in turn each of these diagnostic tools. In the following discussion, a "resonant transition" corresponds to the absorption of a photon from the ground state and re-emission to the same lowest level of the ground state, while a "non-resonant transition" takes place when the photon is re-emitted to an excited level of a ground state that has multiple levels due to fine structure splitting (non-resonant transitions are traditionally labeled with a* such as Fe $\mathrm{II}^{*}$ ). Below, we adopt the usual nomenclature for absorption systems based on their hydrogen column density $N_{\mathrm{H}}$ : damped Ly $\alpha$ absorbers or "DLAs" have $N_{\mathrm{H}}>2 \times 10^{20} \mathrm{~cm}^{-2}$ and Lyman Limit Systems or LLS have $N_{\mathrm{H}}>10^{16.2} \mathrm{~cm}^{-2}$.

\section{$3.3 .1 \mathrm{HI} 21 \mathrm{~cm}$}

Most of the hydrogen in space far from the sources of ionization is neutral and in the ground state, but this ground state exhibits hyperfine splitting. The "spin-flip" H I $21-\mathrm{cm}(1.420-\mathrm{GHz})$ emission line is produced when the slightly excited electron in neutral hydrogen flips its spin to enter a lower energy state, going from parallel to anti-parallel with the spin of the proton. The so-called spin temperature, $T_{s}$, sets the relative populations of these two energy levels. H I $21 \mathrm{~cm}$ has been detected in several cool outflows, either in emission or in absorption against the radio continuum. This technique has been reviewed by Morganti and Oosterloo (2018) in a general extragalactic context, so here we only focus on the essentials relevant to cool outflows. The strength of H I $21 \mathrm{~cm}$ is generally a direct measure of the H I column density, although corrections apply at very low densities (e.g., Liszt 2001). When H I $21 \mathrm{~cm}$ is in emission,

$$
N_{\mathrm{H} \mathrm{I}}^{*}=1.823 \times 10^{18} \mathrm{~cm}^{-2} \int T_{b}(v)[\mathrm{K}] d v\left[\mathrm{~km} \mathrm{~s}^{-1}\right],
$$

where $T_{b}(\mathrm{v})$ is the observed profile of the $\mathrm{H}$ I emission line as a function of velocity, so the $\mathrm{H}$ I equivalent width $\mathrm{EW}(\mathrm{H} \mathrm{I})=\int T_{b}(v) d v$. This expression underestimates the actual value of $N_{\mathrm{HI}}$ if the $\mathrm{H}$ I emission is not optically thin. The ratio $N_{\mathrm{HI}} / N_{\mathrm{HI}}^{*}=$ $\tau_{\mathrm{HI}} /\left[1-\exp \left(-\tau_{\mathrm{HI}}\right)\right]$, where $\tau_{\mathrm{HI}}$ is given by the following equation (Fukui et al. 2015),

$$
\tau_{\mathrm{H} \mathrm{I}}=\frac{N_{\mathrm{H} \mathrm{I}}\left[\mathrm{K} \mathrm{km} \mathrm{s}^{-1}\right]}{1.823 \times 10^{18}} \times \frac{1}{T_{s}[K]} \times \frac{1}{\Delta v_{\mathrm{H} \mathrm{I}}\left[\mathrm{km} \mathrm{s}^{-1}\right]} .
$$

When $\mathrm{H} \mathrm{I}$ is seen in absorption against the continuum,

$$
N_{\mathrm{HI}}=1.823 \times 10^{18} \mathrm{~cm}^{-2} T_{s}[K] \int \tau(v) d v\left[\mathrm{~km} \mathrm{~s}^{-1}\right]
$$


In this expression, the optical depth is defined as $\tau(v)=-\ln \left[1+\Delta T(v) / c_{f} T_{c}\right]$, where $\Delta T(v)$ is the observed absorption spectrum, expressed in terms of the brightness temperature relative to $T_{c}$, the brightness temperature in the continuum, and $c_{f}$ is the covering factor, i.e. the fraction of the continuum source that is covered by the H I absorbers. If we make the (generally reasonable) assumption that $\tau(\mathrm{v})<<1$, this expression becomes

$$
N_{\mathrm{HI}}=1.823 \times 10^{18} \mathrm{~cm}^{-2} \frac{T_{s}}{c_{f} T_{c}} \int|\Delta T(v)| d v\left[\mathrm{~km} \mathrm{~s}^{-1}\right],
$$

The H I column density derived from the absorption feature thus depends on the spin temperature and optical depth, which itself depends on the covering factor. These dependencies make the $\mathrm{H}$ I column densities derived from $\mathrm{H}$ I $21 \mathrm{~cm}$ absorption line more uncertain than those derived from the H I $21 \mathrm{~cm}$ emission line. $T_{s}$ is typically $\sim 100 \mathrm{~K}$ unless the absorbing gas is affected by the radiation field of a powerful nearby continuum source (e.g., AGN or starburst). In that case, $T_{s}$ will depend on the density of the absorbing gas and the location of this gas relative to the source(s) of radiation (e.g., Bahcall and Ekers 1969).

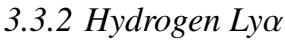

In most astrophysical environments, the electron of neutral hydrogen is in its ground electronic state $(n=1)$ so the resonance electric-dipole transition between the ground state and the first energy level $(n=2)$, H I Ly $\alpha$ 1212 , is the strongest line in the spectrum. The detection of this line in absorption is a powerful tracer of the column density of neutral hydrogen along the line of sight from the background continuum source to the observer. In the linear regime, we simply have $N(\mathrm{H} \mathrm{I})=W(\operatorname{Ly} \alpha)_{\lambda} /(\lambda f)$, where $f, \lambda$, and $W(\operatorname{Ly} \alpha)_{\lambda}$ are the oscillator strength, wavelength (in vacuum, in the rest frame), and equivalent width of $\operatorname{Ly} \alpha$. Unfortunately, this simple equation only applies when the optical depth is $<<1$, corresponding to $N(H I) \lesssim 10^{14} \mathrm{~cm}^{-2}$. Above these values, the two quantities follow a curve-of-growth relation, with a flat portion where $\mathrm{EW} \propto \sqrt{\ln (N)}$ for $N(\mathrm{H} \mathrm{I}) \sim 10^{14}-10^{18} \mathrm{~cm}^{-2}$ and a square-root portion EW $\propto \sqrt{N}$ above $10^{18} \mathrm{~cm}^{-2}$ (e.g., Hummels et al. 2017). In most circumstances, Ly $\alpha$ is not in the linear regime and detailed fitting of the damping wings is required to derive accurate $N(\mathrm{H} \mathrm{I})$. An alternate way of deriving $N(H I)$ is to avoid Ly $\alpha$ altogether and use the fainter lines of the Lyman series from $\sim 926$ down to $\sim 918 \AA$, ignoring the higher wavelength transitions due to blending (e.g., Zech et al. 2008). A standard curve-of-growth analysis can then be used to derive $N(\mathrm{H} \mathrm{I})$.

We have so far ignored sources (e.g., H II regions, AGN) or sinks (e.g., dust, $\mathrm{H}_{2}$ ) of Ly $\alpha$ photons, but these need to be taken into account when evaluating the hydrogen column densities. Ly $\alpha$ photons may be produced through a number of processes: (1) recombination radiation following hydrogen photoionization by a nearby AGN or star-forming galaxy, (2) collisional excitation, (3) collisional ionization followed by recombination radiation, and (4) Ly $\alpha$ scattering (sometimes called "fluorescence" in the literature) of the photons produced in the AGN or within the ISM of a star-forming galaxy. In the first case of recombination radiation, the strength of Ly $\alpha$ is a measure 
of the amount of ionized material rather than neutral material:

$$
M_{\text {ionized }}=C_{e} \frac{m_{p} L(L y \alpha)}{n_{e} \alpha_{L y \alpha}^{\mathrm{eff}} h v_{L y \alpha}},
$$

where $\alpha_{L y \alpha} \sim 2 \times 10^{-13} T_{4}^{-1.26} \mathrm{~cm}^{3} \mathrm{~s}^{-1}$ is the effective Case B Ly $\alpha$ recombination coefficient (Cantalupo et al. 2008), and $C_{e} \equiv\left\langle n_{e}^{2}\right\rangle /\left\langle n_{e}\right\rangle^{2}$ is the electron density clumping factor, which can be assumed to be of order unity on a cloud-by-cloud basis (i.e. each cloud has uniform density). Note, however, that for the reasons discussed below, $\mathrm{H} \alpha$ or strong collisionally excited metal lines, such as [O III] $\lambda 5007$, are more reliable indicators of the ionized mass of outflows than Ly $\alpha$. So, for completeness, we list the relations between the luminosities of these lines and the ionized masses ${ }^{2}$ :

$$
\begin{aligned}
& M_{\text {ionized }}=3.3 \times 10^{8} \frac{C_{e} L_{44}(H \alpha)}{n_{e, 3}} M_{\odot}, \\
& M_{\text {ionized }}=5.3 \times 10^{7} \frac{C_{e} L_{44}([O \text { III } 5007])}{n_{e, 3} 10^{[O / H]}} M_{\odot},
\end{aligned}
$$

where $L_{44}(\mathrm{H} \alpha)$ and $L_{44}([\mathrm{O}$ III] 5007) are respectively the luminosities of $\mathrm{H} \alpha$ and [O III] $\lambda 5007$, normalized to $10^{44} \mathrm{erg} \mathrm{s}^{-1}, n_{e, 3}$ is the average electron density, normalized to $10^{3} \mathrm{~cm}^{-3}, C_{e}$ is the electron density clumping factor defined earlier, and $10^{[\mathrm{O} / \mathrm{H}]}$ is the oxygen-to-hydrogen abundance ratio relative to the solar value.

Ly $\alpha$ emission via collisional excitation may be more important than recombination emission if $T \simeq 2-5 \times 10^{4} \mathrm{~K}$ and the medium is partially ionized (i.e. both neutral hydrogen and free electrons are present; Cantalupo et al. 2008). For temperatures above $\sim 10^{5} \mathrm{~K}$, collisional ionization dominates over collisional excitation. An external source of heating (e.g., AGN, starburst, shocks) is needed to balance radiative losses due to collisional excited $\operatorname{Ly} \alpha$, otherwise the electron temperature quickly drops, effectively stopping the production of $\operatorname{Ly} \alpha$ emission. If collision excitation dominates, one can estimate the neutral gas mass:

$$
M_{\text {neutral }}=C_{\mathrm{e}, \mathrm{HI}} \frac{m_{p} L(\mathrm{Ly} \alpha)}{n_{e} q_{\mathrm{Ly} \alpha}^{\mathrm{eff}} h \nu_{\mathrm{Ly} \alpha}},
$$

where $q_{L y \alpha}^{\text {eff }}$ is the effective collisional excitation coefficient for Ly $\alpha$ emission and is a steep function of $T$ (e.g., Giovanardi et al. 1987), and $C_{\mathrm{e}, \mathrm{HI}} \equiv\left\langle n_{e} n_{\mathrm{HI}}\right\rangle /\left\langle n_{e}\right\rangle\left\langle n_{\mathrm{H} \mathrm{I}}\right\rangle$ is the clumping factor of the partially ionized gas, which can be assumed to be of order unity on a cloud-by-cloud basis (i.e. each cloud has uniform H I and electron densities).

The scattering scenario is only important when the gas is not (photo-)ionized i.e. the density of neutral hydrogen is high enough for the Ly $\alpha$ depth at line center,

$$
\tau_{0}=3.3 \times 10^{-14} T_{4}^{-1 / 2} N(H I),
$$

to be significant. The source of radiation must therefore produce copious amounts of $\operatorname{Ly} \alpha$ and continuum photons slightly blueward of $\operatorname{Ly} \alpha$, but not below $912 \AA$ to

\footnotetext{
2 Both eq. (28) and (29) assume $T \sim 10^{4} \mathrm{~K}$, while eq. (29) further assumes that $n_{e} \lesssim 7 \times 10^{5} \mathrm{~cm}^{-3}$ the critical density associated with the [O III] 5007 transition above which collisional de-excitation becomes significant.
} 
avoid photoionization. During scattering, the Ly $\alpha$ photons will perform a random walk both in space and frequency, and will only be able to escape from the outflow when scattered in the wings of the line profile.

Dust may absorb Ly $\alpha$ photons as they perform their random walk. The optical depth due to dust $\tau_{d}=N_{H} \sigma_{d} \simeq 0.72 \times 10^{-21} N_{H}$ at the wavelength of Ly $\alpha$ for a LMC-type dust (Laursen et al. 2009). The energy absorbed will increase the temperature of the dust grain, which will cause the grain to re-radiate in the infrared. The impact of dust increases with increasing $N(\mathrm{H}$ I) because Ly $\alpha$ scattering becomes more important. This effectively lengthens by a factor of $\tau_{0}$ the path of the Ly $\alpha$ photons through the dusty medium and thus increases the chance that Ly $\alpha$ is absorbed by the dust. The dust grain can also scatter the Ly $\alpha$ photon with a probability given by its albedo, $A_{s}=0.32$ at the $\mathrm{Ly} \alpha$ wavelength for dust made mainly of graphite and silicates (Li and Draine 2001). Finally, in cases where the source of $\operatorname{Ly} \alpha$ is surrounded by molecular gas, Ly $\alpha$ may pump lines of the $\mathrm{H}_{2}$ Lyman bands that are $<100 \mathrm{~km} \mathrm{~s}^{-1}$ redward of Ly $\alpha$, and thus may be destroyed (e.g., Neufeld 1990).

Overall, the calculation of the emergent $\operatorname{Ly} \alpha$ emission is complex, since it depends on the details of the gas structure, ionization, and kinematics as well as the relative distribution of the sources and sinks of $\operatorname{Ly} \alpha$ photons. A quantitative interpretation of the observed Ly $\alpha$ profiles generally requires three-dimensional radiative transfer calculations where all of the relevant spatial and kinematic information is provided as input. Remarkably, simple expanding spherical shell models (e.g., Verhamme et al. 2008; Dijkstra 2017; Gronke 2017) provide a relatively good match to the observed integrated Ly $\alpha$ profiles of outflowing gas in high-z galaxies, though not always (Dijkstra 2017). More recent simulations now consider well resolved turbulent cloud structures (Kimm et al. 2019) and the impact of cosmic rays on the Ly $\alpha$ spectrum (Gronke et al. 2018).

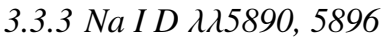

The Fraunhofer $\mathrm{D}_{1}$ and $\mathrm{D}_{2}$ lines of neutral sodium at 5896 and $5890 \AA$, first identified in the solar spectrum, are by far the strongest lines from this element in the visible range. Since the first ionization potential of sodium is only $5.14 \mathrm{eV}(\sim 2400 \AA)$, Na I D is an excellent tracer of neutral hydrogen, but dust is generally required to provide shielding for Na I against non-ionizing UV sources. Na I D is often detected in absorption against the continuum of galaxies, and indeed a correlation is seen between the strength of the $\mathrm{Na} I \mathrm{D}$ absorption feature and dust extinction traced by the color excess, $E(\mathrm{~B}-\mathrm{V})$ (Veilleux et al. 2005). Na I D has also been observed as redshifted emission in a few objects (Phillips 1993; Rupke and Veilleux 2015; Roberts-Borsani and Saintonge 2019), in which case it can be used in combination with the blueshifted absorption feature to further constrain the geometry of the cool outflow (Prochaska et al. 2011).

The general expression for the intensity of a single absorption line, assuming a continuum level of unity, is

$$
I(\lambda)=1-C_{f}(\lambda)+C_{f}(\lambda) e^{-\tau(\lambda)}
$$


where $C_{f}$ is the line-of-sight covering fraction or the fraction of the background source producing the continuum that is covered by the absorbing gas. The optical depth $\tau(\lambda)$ can be expressed as a Gaussian under the curve-of-growth assumption (i.e. a Maxwellian velocity distribution)

$$
\tau(\lambda)=\tau_{0} e^{-\left(\lambda-\lambda_{0}\right)^{2} /\left(\lambda_{0} b / c\right)^{2}}
$$

where $\tau_{0}, \lambda_{0}$, and $b$ are the central optical depth, central wavelength, and Doppler width $(b=\sqrt{2} \sigma=\mathrm{FWHM} /[2 \sqrt{\ln 2}])$, respectively. Note that Gaussians in optical depth translate into observable non-Gaussian intensity profiles for optical depths greater than unity. $C_{f}$ is generally unity in the case for quasar, stellar or GRB sightlines through the ISM, CGM, or IGM, since the background light source in each of these cases can be approximated as a point source. However, when the background light source subtends a significant angle on the sky relative to the absorbing gas (as in the case that concerns us where the background continuum source is the host galaxy itself), $C_{f}$ may depend on wavelength (velocity) and position (if the absorbing gas is spatially resolved). In these cases, a degeneracy arises when solving for optical depth and covering fraction for a single line. This degeneracy is typically broken by observing two or more transitions in the same ion, with the same lower level, whose relative optical depths can be computed exactly from atomic physics. This is the origin of the doublet ratio method where it can be shown that the ratio of the equivalent widths of the two lines in the doublet is a monotonic function of the central optical depth of either line (see equation 2-39 and Table 2.1 in Spitzer 1968) as long as $C_{f}$ is independent of velocity and the velocity distribution is Maxwellian. With knowledge of the optical depth, the covering fraction then follows from the residual intensity of the doublet lines.

Unfortunately, this method cannot be applied to Na I D in cool outflows where the lines in the doublet are usually blended. Rupke et al. (2005b) discussed the more general case where the lines in the doublet are blended together and are produced by two distinct velocity components that either completely, partially, or do not, overlap with each other. For partially overlapping velocity components, Eq. (32) becomes

$$
I(\lambda)=\left[1-C_{f, 1}(\lambda)+C_{f, 1}(\lambda) e^{-\tau_{1}(\lambda)}\right] \times\left[1-C_{f, 2}(\lambda)+C_{f, 2}(\lambda) e^{-\tau_{2}(\lambda)}\right]=I_{1}(\lambda) I_{2}(\lambda \gamma(34)
$$

and the total covering fraction of the continuum source at a given wavelength is

$$
C_{f, \text { total }}=C_{f, 1}+C_{f, 2}-C_{f, 1} C_{f, 2}
$$

Once the optical depth profile $\tau_{\lambda}(\lambda)$ is known, one can derive the column density producing the absorption line. The general expression for the column density of an ionic species is (Spitzer 1978)

$$
\begin{aligned}
N & =\frac{m_{e} c}{\pi e^{2} f \lambda_{0}} \int \tau(v) d v \\
& =\frac{3.768 \times 10^{14} \mathrm{~cm}^{-2}}{f \lambda_{0}} \int \tau(v) d v
\end{aligned}
$$


where $f$ and $\lambda_{0}$ are the oscillator strength and wavelength (in vacuum, in the rest frame) of the transition. For neutral sodium, this expression becomes

$$
N(\mathrm{Na} \mathrm{I})=3.56 \times 10^{11}\left(\tau_{1, c} b\right) \mathrm{cm}^{-2},
$$

where $\tau_{1, c}$ is the central optical depth of the $\mathrm{Na}$ I $\mathrm{D}_{1} \lambda 5896$ line and $b$ is the Doppler parameter in $\mathrm{km} \mathrm{s}^{-1}$.

Stellar absorption from late-type stars in the underlying galaxy spectrum may contribute to the observed profile of $\mathrm{Na} I \mathrm{D}$. The expected stellar contribution to $\mathrm{Na}$ $\mathrm{I} \mathrm{D}$ has been estimated in the past by scaling the equivalent width of the $\mathrm{Mg} \mathrm{I} \mathrm{b}$ triplet at 5167, 5173, and $5185 \AA$. This is possible because $\mathrm{Na}$ and $\mathrm{Mg}$ are created in stars by similar mechanisms and they have similar first ionization potentials (5.14 $\mathrm{eV}$ for $\mathrm{Na}, 7.65 \mathrm{eV}$ for $\mathrm{Mg}$ ). There is thus a correlation between the stellar equivalent widths of $\mathrm{Mg}$ I b and Na I D in galaxy spectra: $W^{*}(\mathrm{Na}$ I D $)=\alpha_{\mathrm{Na}} W^{*}(\mathrm{Mg} \mathrm{I}$ b $)+$ $\beta_{\mathrm{Na}}$ where $\left(\alpha_{\mathrm{Na}}, \beta_{\mathrm{Na}}\right)=(0.5,0)$ in U/LIRGs (Rupke et al. 2005c) and $(0.685,0.8)$ in emission-line galaxies (Alatalo et al. 2016). Another weaker stellar feature may also be present at a rest wavelength of $5857.5 \AA, 33 \AA\left(1700 \mathrm{~km} \mathrm{~s}^{-1}\right)$ blueward of the $\mathrm{D}_{2}$ line. A more important contaminant near this region is the He I $\lambda 5876$ recombination emission line from ionized gas; this line is generally fit with one or two Gaussians in intensity.

Once $N(\mathrm{Na}$ I) has been derived, the column density of hydrogen, $N(\mathrm{H})$, can be estimated from $N(\mathrm{Na}$ I) given a metallicity and ionization fraction (Rupke et al. 2005b):

$$
N(\mathrm{H})=N(\mathrm{Na} \mathrm{I}) X_{\mathrm{Na}^{0}}^{-1} 10^{-\left(A_{\mathrm{Na}}+B_{\mathrm{Na}}\right)},
$$

where $X_{\mathrm{Na}^{0}} \equiv N\left(\mathrm{Na}\right.$ I) / $N(\mathrm{Na})$ is the fraction of $\mathrm{Na}$ that is neutral $\mathrm{Na} \mathrm{I}, A_{\mathrm{Na}} \equiv$ $\log [N(\mathrm{Na}) / N(\mathrm{H})]_{\text {gas }}$ is the gas-phase $\mathrm{Na}$ abundance relative to $\mathrm{H}$, and $B_{\mathrm{Na}} \equiv \log [N(\mathrm{Na}) / N(\mathrm{H})]_{\mathrm{gas}}-$ $\log [N(\mathrm{Na}) / N(\mathrm{H})]_{\text {total }}$ is the level of depletion of Na onto dust. Typically, Milky-Way like metal abundance of sodium $\left(A_{\mathrm{Na}}=-5.69\right.$; Savage and Sembach 1996) and depletion factor of sodium onto dust $\left(B_{\mathrm{Na}}=-0.95\right.$; Savage and Sembach 1996) are adopted in this expression. The Na neutral fraction $X_{\mathrm{Na}^{0}}$ is uncertain. A value $X_{\mathrm{Na}^{0}}=0.1$ has been measured toward Galactic stars (Stokes 1978) and also a cold HI cloud in NGC 3067 (Stocke et al. 1991), but this number could be substantially smaller in the more extreme environment of an AGN or star-forming galaxy, therefore resulting in a larger $N(\mathrm{H})$.

Direct measurements of the $\mathrm{H}$ I columns from $21-\mathrm{cm}$ radio data may provide an independent check on the Na-based $N(\mathrm{H})$. For instance, Teng et al. (2013) detected two $\mathrm{H} \mathrm{I}$ absorption components at the same velocities as the $\mathrm{Na}$ I outflow components in a ULIRG (Rupke and Veilleux 2015) and derived $N(\mathrm{H})=10^{22} \mathrm{~cm}^{-2}$, the same column inferred from $\mathrm{Na} I \mathrm{D}$ in the dustiest regions of this galaxy. Other more indirect measures of $N(\mathrm{H})$ in cool outflows, based on reddening measurements of the underlying host galaxy light, have brought additional support for these correction factors (Rupke and Veilleux 2013b), although they likely remain uncertain to within factors of $\pm 2-3$.

Note finally that $\mathrm{Na}$ I D has also been seen in emission in a few cool outflows (see Sec. 5.1). Since the Na I D emission lines are resonant transitions like Ly $\alpha$, they are subject to scattering and "fluorescence" by the cool gas and thus can also be used 
to trace this component of the outflows. Simple models of metal-line absorption and emission from cool gas outflows that track the scattered and fluorescent photons in a spherical homogenously expanding wind have been discussed in Prochaska et al. (2011), and these are directly applicable to the observed Na I D P-Cygni like profiles (e.g., Rupke and Veilleux 2015).
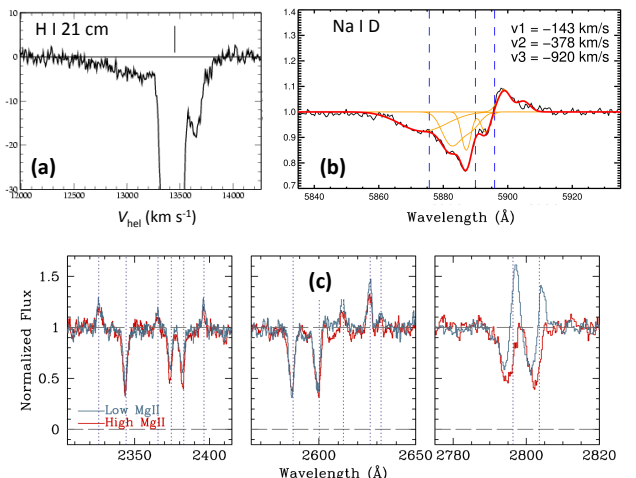

$[\mathrm{CI}]^{3} \mathrm{P}_{1}-\mathrm{P}^{\mathrm{P}} \mathrm{P}^{-10}$
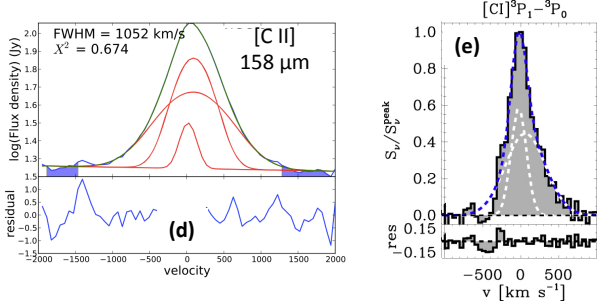
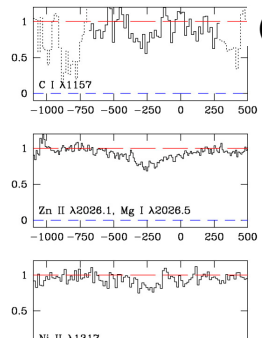

$$
. \text { Ni II } 11317
$$
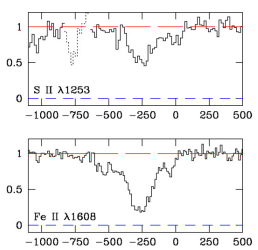

$-1000-750-500-250 \quad 0 \quad 250 \quad 500$

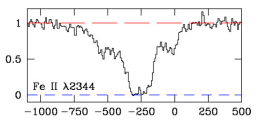

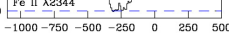
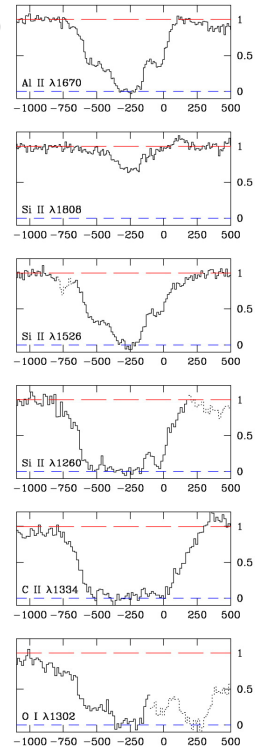

Fig. 6 Some of the diagnostic tools used to study neutral-atomic gas outflows. (a) H I $21 \mathrm{~cm}$ from, (b) optical Na I D, (c) NUV Mg II and Fe II, (d) FIR [C II] $158 \mu \mathrm{m}$, (e) mm-wave [C I], and (f) FUV lines from neutral and low-ionization elements. Images reproduced with permission from (a) Morganti et al. (2005), copyright by ESO; and (b) Rupke et al. (2017), (c) Erb et al. (2012), (d) Janssen et al. (2016), (e), Cicone et al. (2018b), (f) Pettini et al. (2002), copyright by AAS.

\subsubsection{UV Lines from Neutral Elements}

UV transitions from abundant neutral chemical elements have been used to probe cool outflows. The strongest features between $1000 \AA$ and $3000 \AA$, most commonly used to study the cool ISM/CGM in the Milky Way and external galaxies, include O I 1302, N I 1134, 1200, C I 1277, 1280, 1329, 1657, and Mg I 2853. Outside ISM environments, these features are generally weak enough that the column densities of these elements may be derived using a simple curve-of-growth analysis of the fitted absorption line profiles against the continuum of the background UV-bright source. These column densities can then be compared with H I column density measurements, derived for instance from the H I $21-\mathrm{cm}$ line emission, to determine the metallicity of the absorbing material, modulo an ionization correction to take into 
account the ionized portion of the gas. The O I/H I ratio is particularly well suited for this measurement because it is only moderately depleted onto dust grains (Jenkins 2009) and, since oxygen and hydrogen have virtually identical first ionization potentials, strong charge-exchange reactions tie the two species together (Field and Steigman 1971; Domgorgen and Mathis 1994). This method has for instance been used in HVCs associated with the nuclear outflow in the Milky Way (e.g., Keeney et al. 2006; Zech et al. 2008; Bordoloi et al. 2017b, see Sect. 4.1.1 below). Since the HI 21-cm observations have a finite beam and the UV observations are effectively infinitesimal, beam-smearing corrections must be applied to take into account potential small-scale structure in the H I beam (e.g., Walter et al. 2017; Jenkins 2009; Bordoloi et al. 2017b). At sufficiently high spectral resolution $(R \gtrsim 30,000)$, it is possible to distinguish between the three fine-structure levels of the ground electronic state of $\mathrm{C} \mathrm{I}$, and use the C I / $\mathrm{C} \mathrm{I}^{*} / \mathrm{CI}^{* *}$ ratios to derive the thermal pressure in the absorbing gas (Jenkins and Tripp 2001; Savage et al. 2017).

\subsubsection{UV lines from Singly Ionized Elements}

Many of the most abundant elements have first ionization potentials that fall below $13.6 \mathrm{eV}$, and therefore are also good tracers of neutral hydrogen. Key elements include $\mathrm{C}^{+}(11.26 \mathrm{eV}), \mathrm{Na}^{+}(5.14 \mathrm{eV}), \mathrm{Mg}^{+}(7.65 \mathrm{eV}), \mathrm{Al}^{+}(5.99 \mathrm{eV}), \mathrm{Si}^{+}(8.15 \mathrm{eV})$, $\mathrm{S}^{+}(10.36 \mathrm{eV}), \mathrm{Ca}^{+}(6.11 \mathrm{eV})$ (and also $\left.\mathrm{Ca}^{++}, 11.87 \mathrm{eV}\right)$, and $\mathrm{Fe}^{+}(7.87 \mathrm{eV})$. The strongest absorption lines between $1000 \AA$ and $4000 \AA$, most commonly used in the study of the cool ISM/CGM in the Milky Way and external galaxies, include Ca II 3935 (K) and 3970 (H), Mg II 2796, 2804, Fe II 2344 (UV3), 2374 + 2383 (UV2), 2587 + 2600 (UV1), Si II 1190, 1193, 1260, 1304, 1527, C II 1334, and Al II 1671. Weaker absorption lines from slightly less abundant but volatile elements that are less subject to dust depletion [e.g., S II 1251, 1254, 1260 from S $^{+}(10.36 \mathrm{eV}), \mathrm{Zn}$ II 2026, 2063 from $\left.\mathrm{Zn}^{+}(9.39 \mathrm{EV})\right]$ can be used in conjunction with the lines from refractory elements (e.g, Savage et al. 2017) to estimate the dust depletion factor in the neutral gas phase of cool outflows.

Several resonant transitions (e.g., Mg II 2796, 2804) and non-resonant opticallythin transitions (e.g., Fe II* 2626, 2612, 2396, 2365, C II* 1335, Si II* 1265, 1309, $1533)$ are also commonly ( $\sim 15-30 \%)$ observed in emission from cool outflows, particularly in $z \sim 1$ galaxies with blue UV slopes and small stellar masses (Erb et al. 2012; Kornei et al. 2013). This line emission can be used in combination with the absorption line to further constrain the properties of the cool outflows (Prochaska et al. 2011). On the other hand, if the spectral resolution is poor, this line emission "infills" the absorption lines of the resonant transitions (e.g., Mg II 2796, 2804), particularly at velocities near the systemic velocity of the galaxy, and thus complicates the analysis of these lines (Zhu et al. 2015). Contamination from stellar absorption features at systemic velocity also needs to be taken into account for the analysis of $\mathrm{Mg}$ II and Fe II. Fits with two separate velocity components for each line are generally used to mitigate these effects (Rubin et al. 2010; Coil et al. 2011). Typically, $\mathrm{Mg}$ II $\left(\mathrm{IP}_{1}=7.65 \mathrm{eV}, \mathrm{IP}_{2}=15.0 \mathrm{eV}\right)$ is assumed to be the dominant ionization state in photoionized $T \sim 10^{4} \mathrm{~K}$ winds (Murray et al. 2007), so an ionization correction factor $X_{\mathrm{Mg}^{+}} \simeq 1 \mathrm{in}$ Eq. (39) is used to derive the total $\mathrm{Mg}$ abundance in the gas phase, 
and a dust depletion corrections $B_{\mathrm{Mg}}=-0.5$ dex is used in galaxies with near-solar abundances (Rubin et al. 2014). For Fe II, $X_{\mathrm{Fe}^{+}}=[0.5-1.0]$ and $B_{\mathrm{Fe}}=[-0.69,-1.0]$ have been used in the literature (Martin et al. 2012; Rubin et al. 2014).

Absorption lines originating from the (collisionally) excited metastable state of these ions (e.g., Fe II*, Si II*) have been detected in several objects, particularly in LOw-ionization Broad Absorption Line (LoBAL) QSOs, and have been used to constrain the electron density in the absorbing material. This information can be used to derive the distance $r$ of the absorber from the central ionizing source via the dimensionless ionization parameter:

$$
U_{H} \equiv \frac{n(h v>13 \mathrm{eV})}{n_{H}}=\frac{Q_{H}}{4 \pi r^{2} n_{H} c},
$$

where $n_{e} \simeq 1.2 n_{H}$ for highly ionized plasma, $c$ is the speed of light, $n(h v>13 \mathrm{eV})$ and $Q_{H}$ are the density and rate of ionizing $(>13.6 \mathrm{eV})$ photons hitting the absorber, respectively. $Q_{H}$ is derived by integrating the UV-soft X-ray portion of the AGN spectral energy distribution that best fits the broad-band data on the object. Dust between the source and the absorber needs to be taken into account in the calculations of $Q_{H} . U_{H}$ is derived independently by comparing the measured column densities with the predictions of photoionization models under the assumption of ionization equilibrium (e.g. Moe et al. 2009; Dunn et al. 2010). This method has more recently been extended to the broad absorption lines from more highly ionized species (e.g., $\mathrm{Si} \mathrm{IV}^{*}$ ) in the more common High-ionization Broad Absorption line (HiBAL) QSOs (e.g. Arav et al. 2013, 2018; Miller et al. 2018; Xu et al. 2018), and for all BAL QSOs in general (Hamann et al. 2019). The timescale of variability of these absorption lines has also been used to constrain the density of the absorbers, hence their distance, assuming it is due to changes in the ionizing radiation incident on the absorbing gas rather than tangential movement of the absorbing gas across the line of sight (Filiz Ak et al. 2012; Wang et al. 2015). In this case, the variability time scale sets a constraint on the ionization or recombination timescale, which depends solely on the incident ionizing continuum and gas density (e.g. He et al. 2019).

\subsubsection{Far-Infrared [C I] and [C II] Emission Lines}

While FIR [C I] and [C II] lines are formally tracers of the neutral-atomic component of cool outflows, they are also excellent tracers of the molecular component so they are instead discussed in Section 3.4.4 below.

\subsection{Diagnostic Tools of the Molecular Gas Phase}

Millimeter and submillimeter-wave techniques can provide excellent direct estimates of the luminosity of $\mathrm{CO}$ and other molecules, as well as their kinematics, and morphology (the latter through interferometric imaging). This information can be converted into estimates for column density, volume density, and mass, momentum, energy, and their fluxes for the molecular phase. The fact that the millimeter/submillimeterwave window is very rich in molecular transitions means observations provide also a unique view of the chemistry and excitation in the outflowing gas. 
(a)
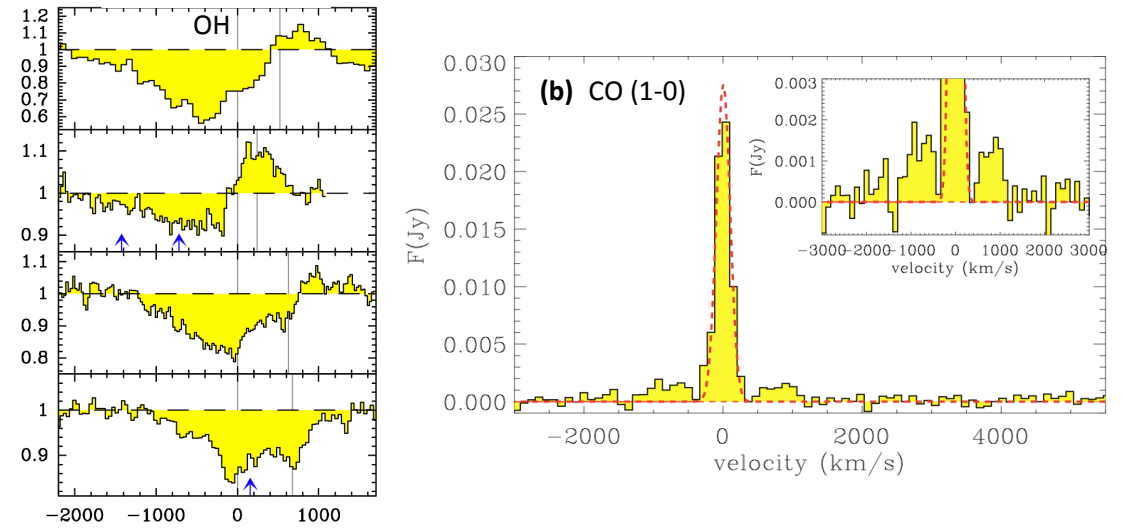

(c)

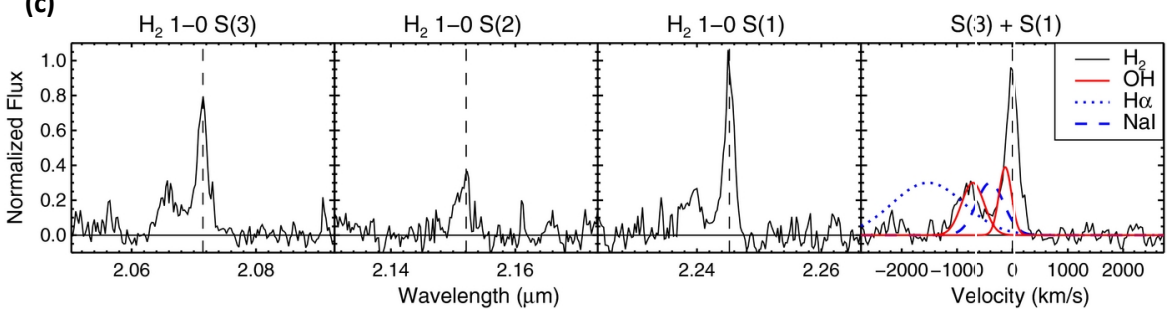

Fig. 7 Some of the diagnostic tools used to study molecular gas outflows. (a) $\mathrm{OH}$ absorption and emission line profile, (b) $\mathrm{CO}$ emission line profile, (c) near-infrared ro-vibrational $\mathrm{H}_{2}$ emission lines. Images reproduced with permission from (a) González-Alfonso et al. (2017b), (c) Rupke and Veilleux (2013a), copyright by AAS; and (b) Cicone et al. (2014), copyright by ESO.

Measurements obtained at single-dish radio telescopes are usually expressed in Rayleigh-Jeans brightness temperature units of Kelvins, corresponding to $T_{B}=I_{v} c^{2} / 2 k v^{2}$, which are meaningful for the angular resolution (beam size) of the observation. In interferometers, the measurements are usually expressed in flux density units. The conversion between temperature surface brightness units and flux density is

$$
T_{B}=1360 \mathrm{~K} \frac{\lambda^{2}}{\theta^{2}} S_{v}
$$

where $T_{B}$ is the Rayleigh-Jeans brightness temperature in $\mathrm{K}, \lambda$ is the wavelength of the observation in $\mathrm{cm}, \theta$ is the full-width at half-maximum of the Gaussian beam of the observation in arcseconds, and $S_{v}$ is the flux density in Jy beam ${ }^{-1}$. The Rayleigh Jeans brightness temperature can be related to the excitation temperature of the transition through

$$
T_{B}=\frac{h v}{k}\left(1-e^{-\tau_{J}}\right)\left(\frac{1}{e^{\frac{h \nu}{k T_{e x, J}}}-1}-\frac{1}{e^{\frac{h \nu}{k T_{c m b}}}-1}\right),
$$


where the Cosmic Microwave Background temperature $T_{c m b}$ is the background against which the emission is measured. The last term is usually a small correction for $z \sim 0$, and it is frequently neglected.

Luminosities are frequently expressed in units of $\mathrm{K} \mathrm{km} \mathrm{s}^{-1} \mathrm{pc}^{2}$, and when using these units they may be noted as $L^{\prime}$ (strictly speaking this is not the usual meaning of luminosity, since $\mathrm{K}$ are units of brightness temperature rather than energy flux per unit time, and $\mathrm{km} \mathrm{s}^{-1}$ are units of velocity rather than units of frequency). In general

$$
L^{\prime}=3.25 \times 10^{7} S_{v} \Delta v v_{o b s}^{-2}(1+z)^{-3} D_{L}^{2}
$$

where $S_{v}$ is the transition flux density in $\mathrm{Jy}, \Delta v$ is the line width in $\mathrm{km} \mathrm{s}^{-1}, v_{o b s}$ is the observed frequency of the transition in $\mathrm{GHz}, z$ is the source redshift, and $D_{L}$ is its luminosity distance in Mpc (Solomon and Vanden Bout 2005).

\subsubsection{Probes of the Volume Density}

Determination of volume densities using millimeter-wave data mostly rely on access to rotational transitions sampling a range of critical densities. In the following discussion we restrict ourselves to transitions between rotational energy levels characterized by a quantum number $J$, although most of it can easily be generalized to other types of transitions. The critical density is the density of the collisional partner at which the chance of spontaneous de-excitation and that of collisional de-excitation are equal, so that $n_{c r}=A_{J} / \gamma_{J}$ where $A_{J}$ is the Einstein $A$ coefficient to spontaneously transition from $J \rightarrow J-1$, and $\gamma_{J}$ is the collisional coefficient for the same transition (note sometimes this is defined using the sum of the coefficients for all possible downward collisional transitions in the denominator). At densities much higher than $n_{c r}$ the populations of the levels are in collisional equilibrium, and follow the distribution corresponding to the Boltzmann equation with an excitation temperature $T_{e x}$ equal to the kinetic temperature of the gas, $T_{K}$. At densities much lower than $n_{c r}$, on the other hand, molecules are preferentially de-excited by spontaneous emission, and their excitation temperature is lower than $T_{K}$.

Over a very wide range of conditions, the brightest transitions from molecular gas at these wavelengths are the rotational transitions of the main isotopologue of carbon monoxide, ${ }^{12} \mathrm{C}^{16} \mathrm{O}$. These transitions of carbon monoxide are excited mostly through collisions with $\mathrm{H}_{2}$ molecules. Radiative trapping will play an important multiplicative role in the excitation if the transition is optically thick, which is easy to achieve since $\mathrm{CO}$ is an abundant molecule that is frequently the largest reservoir of carbon in the gas phase. The critical density for the ground rotational transition of $\mathrm{CO}$ (at $v=$ $115.27 \mathrm{GHz}$ ) to collisions with molecular gas is $n_{c r}\left(\mathrm{H}_{2}\right) \approx 2000 T_{100}^{-0.019} \mathrm{~cm}^{-3}$ (Yang et al. 2010). The excitation is such that bright emission usually requires densities that are similar to or larger than $n_{c r}$ (e.g., Leroy et al. 2017). In species like CO that are abundant and optically thick, radiative trapping acts to reduce the effective critical density by a factor of approximately $\left(1-e^{-\tau_{J}}\right) / \tau_{J}$, which is a significant correction when the optical depth $\tau_{J}$ of the $J \rightarrow J-1$ transition is large.

Critical densities scale approximately as $n_{c r} \propto \mu^{2}$, where $\mu$ is the electronic dipole moment of the molecular species, so that molecules with higher $\mu$ require 
higher volume densities to be excited collisionally. For a given molecular species $n_{c r} \propto J^{3}$, so that higher transitions have higher critical densities. Among the abundant molecules with bright millimeter-wave transitions, $\mathrm{HCN}, \mathrm{HCO}^{+}$, and $\mathrm{CS}$ have high dipole moments and are usually employed as tracers of high density gas. For example, the ground rotational transition of $\mathrm{HCN}$ at $v=88.63 \mathrm{GHz}$ has $n_{c r}\left(\mathrm{H}_{2}\right) \approx$ $2.67 \times 10^{6} T_{100}^{+0.349} \mathrm{~cm}^{-3}$ (Dumouchel et al. 2010). Therefore, the HCN/CO ratio is, to first order, an indicator of density ${ }^{3}$. Comparison across molecular species, however, can be affected by their relative abundances determined by the chemistry. Moreover, some molecules can be excited by more than one collisional partner. For example, $\mathrm{HCN}$ has a cross-section to collisions with electrons that is $10^{5}$ times larger than for collisions with $\mathrm{H}_{2}$ molecules, which may affect its use as a density tracer in regions where electron abundance is high (Goldsmith and Kauffmann 2017). Note also that molecules such as HCN may be affected by infrared radiative excitation and pumping, which can impact the interpretation of the molecular line emission. HCN (and also other molecules) has degenerate bending modes in the infrared. The molecule absorbs infrared photons to the bending mode (its first vibrational state) and then it can decay back to the ground state via its P- or R-branch. In this way, a vibrational excitation may produce a change in the rotational state in the ground level and can be treated (effectively) as a collisional excitation in the statistical equations (e.g. Carroll and Goldsmith 1981; Aalto et al. 2007)

The presence of bright emission from one of the high dipole moment species usually is taken to suggest that the volume density is similar to or higher than the critical density of the transition. A full treatment of the gas density requires solving the detailed balance equations to establish the populations of each rotational level. As we discuss below, this can provide simultaneous constraints on volume density, column density, and temperature.

\subsubsection{Probes of the Column Density and Optical Depth}

The optical depth of a rotational transition relates to the column density in its upper level, $N_{J}$, and velocity width, $\Delta v$, through

$$
\tau_{J}=\frac{8 \pi^{3}}{3 h} \mu^{2} \frac{J}{2 J+1}\left(e^{h v_{J} / k T_{e x, J}}-1\right) \frac{N_{J}}{\Delta v},
$$

where $\mu$ is the electric dipole moment, and $h$ and $k$ are Planck's and Boltzmann's constants respectively. In Local Thermodynamic Equilibrium (LTE, implying $T_{e x, J}=$ $T_{e x} \approx T_{K}$ ), the population of a level, $N_{J}$, and the total column density, $N$, are related through the partition function $Q\left(T_{e x}\right)$ and the corresponding Boltzmann factor,

$$
N=\frac{Q\left(T_{e x}\right)}{2 J+1} N_{J} e^{\frac{h \nu}{k T_{e x}}},
$$

where $Q\left(T_{e x}\right)=2 k T_{e x} / h v$ is a good approximation for $T_{e x} \gg h v / k$. There are a number of different methodologies for measuring optical depths. For a much more

\footnotetext{
3 Just as for $\mathrm{CO}$, high opacities can impact the critical density for e.g. HCN which may be very abundant in warm regions. In addition, the critical density can be strongly reduced (by factors 4-6) in regions of high temperature. This requires a multi-level treatment of the critical density.
} 
extensive discussion on using molecular measurements to estimate column density see Mangum and Shirley (2015).

A good way to estimate the optical depth for an emission line, and consequently have a good handle on the column density of the gas, is to obtain measurements in an optically thin isotopologue of known abundance. If the ratio of column densities of the optically thick to the optically thin isotopologue is $R$, the intensity ratio $r$ of the same transition observed in an optically thick $(R \tau \gg 1)$ to an optically thin $(\tau \ll 1)$ isotopologue will be

$$
r \approx \frac{\left(1-e^{-R \tau}\right) T_{\text {thick }}}{\left(1-e^{-\tau}\right) T_{\text {thin }}} \approx \frac{1-e^{-R \tau}}{1-e^{-\tau}} \sim \frac{1-e^{-R \tau}}{\tau} .
$$

For example, the observed ratio of ${ }^{12} \mathrm{CO} /{ }^{13} \mathrm{CO}$ intensities is frequently observed to be $r \sim 10$, while the abundance ratio is closer to $R \approx 50$ at the Solar circle (Wilson and Rood 1994). Selective photodissociation effects, chemical fractionation, and changes in the ${ }^{13} \mathrm{C} /{ }^{12} \mathrm{C}$ ratio all possibly impact the calculation, as well as the implicit assumption that both lines emerge from the same parcel of gas. This suggests that the typical optical depth of the ${ }^{12} \mathrm{CO}$ emission is $R \tau \sim 5$. Note that this assumes the excitation temperature is similar for both isotopologues, to cancel out its ratio. This is not necessarily correct, particularly in the presence of temperature gradients: the relevant excitation temperature for the optically thick isotopologue is that at the $R \tau \sim 1$ surface, while for an optically thin isotopologue what matters is the temperature throughout the medium. For an internally heated cloud, for example, where the temperature increases toward the center, the optically thick isotopologue may reach optical depth of unity in a colder region far from the center of the cloud. As a consequence the ratio of excitation temperatures will be $T_{\text {thick }} / T_{\text {thin }}<1$, and neglecting this will result in too large an inferred optical depth.

Sometimes it is not possible to detect an optically thin isotopologue in a reasonable amount of time, because the emission is likely to be very weak. Using Eq. (44) it is possible to show that in LTE the ratio of Rayleigh-Jeans brightness temperature of two consecutive rotational transitions $r_{J}=T_{J} / T_{J-1}$ that fill the same area of the beam will be

$$
r_{J}=\left(\frac{J}{J-1}\right)^{2} e^{-\frac{h v}{k T_{e x}}}
$$

for optically thin emission, which for gas with $T_{K} \approx T_{e x}>h v_{J} / k$ is $r_{J} \approx J^{2} /(J-$ $1)^{2}$. For optically thick emission, under the same conditions $r_{J} \approx 1$. This opens the possibility of using measurements of bright line ratios to estimate emission optical depth.

\subsubsection{Probes of the Temperature}

Under the frequent assumption of optically thick emission, the surface brightness of the $\mathrm{CO}$ emission becomes a probe of the excitation temperature of the transition, $T_{e x, J}$, which at densities higher than the effective critical density approaches the kinetic temperature of the gas, $T_{K}$. Obtaining a good estimate requires resolving the 
$\mathrm{CO}$ emission, which is not always possible, otherwise the surface brightness measurement represents the true brightness times an emission dilution factor (the ratio of solid angle $\Omega$ occupied by the emission to that of the telescope beam) that can be very small and uncertain. Thus the observed brightness temperature always provides a lower limit to the gas temperature (unless very unusual excitation and/or radiative transfer conditions occur).

Because it is the combined effects of volume density, column density, and temperature that affect the excitation, the most comprehensive way to constrain these parameters requires modeling the spectral line energy distribution (SLED) across several rotational transitions in perhaps more than one molecular species. Because rotational transitions are equally spaced in frequency, the energy of the upper level $J$ of a rotational transition is $E_{J}=h v_{1} J(J+1) / 2$, and the SLED will be particularly sensitive to the temperature of the gas as long as the highest transition probed has $E_{J} / k$ larger than the gas temperature (see for example, Weiß et al. 2007; Israel et al. 2015). The modeling is usually accomplished by solving the detailed balance equations assuming a large velocity gradient. An example of a widely available code to do this is RADEX (van der Tak et al. 2007).

In general accurate temperature measurements may be derived from emission from symmetric top molecules, such as $\mathrm{NH}_{3}$ (e.g., Mangum et al. 2013). The relative intensity of the rotational ground-state inversion transitions for different $K$-ladders provides a direct measurement of the kinetic temperature. This is because the exchange of population between the $K$-ladders occurs via collisional processes. Asymmetric rotor molecules, such as $\mathrm{H}_{2} \mathrm{CO}$, can also be useful temperature probes (e.g., Mangum and Wootten 1993). However, to detect emission from such rarer molecules in outflows, requires very deep and sensitive observations.

\subsubsection{Estimating the Masses and Mass-Outflow Rates}

The determination of mass usually relies on using an appropriate mass-to-light ratio for a bright line (although it is also possible to use the dust continuum assuming a dust-to-gas ratio). The resulting equation is

$$
M_{m o l}=\alpha_{X} L_{X}^{\prime},
$$

where $\alpha_{X}$ is the mass-to-light ratio for species $X$, and $L_{X}^{\prime}$ is its luminosity in the units of Eq. (43). Note that, frequently, the molecular mass $\mathrm{M}_{m o l}$ is not just that of $\mathrm{H}_{2}$ but it includes a correction for the cosmic abundance of He by mass $(\sim 1.36)$, although this is not entirely consistent across authors.

- Using CO Emission to Estimate Outflow Mass. The ground transition of ${ }^{12} \mathrm{C}^{16} \mathrm{O}$ is frequently used for this purpose, even though it is optically thick. For a full discussion on the foundations and the caveats of this approach, see Bolatto et al. (2013b). Here we will summarize a few relevant equations.

For optically thick emission from a self-gravitating cloud (or an ensemble of clouds), where the velocity dispersion is a reflection of self-gravity, the mass-tolight ratio of the ground rotational transition has been calibrated to be $\alpha_{\mathrm{CO}} \approx 4.4$ $\mathrm{M}_{\odot}\left(\mathrm{K} \mathrm{km} \mathrm{s}^{-1} \mathrm{pc}^{2}\right)^{-1}$ for large $\left(\mathrm{M}_{m o l}>10^{5} \mathrm{M}_{\odot}\right)$ Giant Molecular Clouds in the disk 
of the Milky Way. This value depends on the average temperature of the molecular gas, the mass of the cloud, and the virial parameter of the object, becoming smaller for hotter clouds, smaller clouds, and less bound clouds. Given that, where we can resolve it, molecular emission in outflows is frequently highly turbulent and it probably arises from clouds that may have experienced a shock or are perhaps heated by conduction by the neighboring hot fluid, it is likely that the "Galactic" $\alpha_{\mathrm{CO}}$ is an overestimate of the true mass-to-light ratio of the gas in a galactic outflow. The lowest likely mass-to-light ratio (and consequently the lowest mass estimate) comes from assuming optically thin emission, for which $\alpha_{\mathrm{CO}} \approx 0.34\left(T_{e x} / 30 \mathrm{~K}\right)$ assuming $\mathrm{CO} / \mathrm{H}_{2}=10^{-4}$ and $T_{e x} \gtrsim 30 \mathrm{~K}$ (see Bolatto et al. 2013a). From these considerations it appears that assuming a typical $\alpha_{\mathrm{CO}} \approx 1 \mathrm{M}_{\odot}\left(\mathrm{K} \mathrm{km} \mathrm{s}^{-1} \mathrm{pc}^{2}\right)^{-1}$ yields reasonably bracketed estimates of molecular masses in outflows from $\mathrm{CO}$ observations.

Since $\alpha_{\mathrm{CO}}$ is defined for the ground transition, when using higher transitions of $\mathrm{CO}$ to determine masses it is necessary to correct their intensities by a brightness temperature line ratio, $r_{J 1}=T_{B}(J \rightarrow J-1) / T_{B}(1 \rightarrow 0)$. The applicable line ratio depends on the excitation conditions in the medium: hotter and denser media will lead in general to higher line ratios, and as we saw in Eq. (47) optically thin emission may also lead to higher line ratios. If the emission is optically thick, however, the ratios are $r_{J 1} \leq 1$, and $r_{J 1} \sim 1$ usually requires temperatures hot enough to populate the $J$ level and densities higher than the effective critical density. In galaxies, typical observed line ratios for bright emission are $r_{21} \sim 0.8$ and $r_{31} \sim 0.67$ (e.g., Rosenberg et al. 2015; Kamenetzky et al. 2016). As the $J$ level increases and the required excitation conditions become more stringent, the ratios become increasingly variable from source to source (e.g., Kamenetzky et al. 2018).

Note that line ratios and SLEDs depend on the units used for their luminosities. When line intensities are expressed in $\mathrm{Jy} \mathrm{km} \mathrm{s}^{-1}$ or equivalent units, there will be an additional factor of $v^{2}$ (see Eq. (41)) in the integrated intensities, resulting in line ratios $r_{J 1} J^{2}$. When line intensities are expressed in $\mathrm{erg} \mathrm{cm}^{-2} \mathrm{~s}^{-1}$ or equivalent units, there will be another factor of $v$ associated with the conversion from velocity to frequency, resulting in line ratios $r_{J 1} J^{3}$.

- Using [CI] Emission to Estimate Outflow Mass. The fine structure lines of atomic carbon, [C I] (1-0) ${ }^{3} \mathrm{P}_{1}-{ }^{3} \mathrm{P}_{0}$ and ${ }^{3} \mathrm{P}_{2}-{ }^{3} \mathrm{P}_{1}$ transitions at $609 \mu \mathrm{m}(492 \mathrm{GHz})$ and 370 $\mu \mathrm{m}(809 \mathrm{GHz})$, have received increased attention in recent years as probes of the cool gas in galaxies. How [C I] compares to $\mathrm{CO}$ in terms of producing reliable molecular masses is still unclear. Papadopoulos et al. (2004) argue on theoretical grounds that the (generally) optically thin [C I] (1-0) line may be a better tracer of the molecular gas than ${ }^{12} \mathrm{CO}$ under typical ISM conditions, especially for diffuse $\left(\sim 10^{2}-10^{3}\right.$ $\mathrm{cm}^{-3}$ ) molecular gas in metal-rich environments far from intense UV sources. Similar conclusions are reached by Offner et al. (2014) and Glover et al. (2015) based on simulations of resolved molecular clouds in conditions similar to those at the Solar circle. Israel et al. (2015), on the other hand, analyze the suitability of CO and [C I] to measure molecular masses using an extensive dataset of 76 ULIRGs and starburst galaxy centers. They conclude that the scatter in molecular mass estimation using $[\mathrm{C} \mathrm{I}]$ in these galaxies is not better than using $\mathrm{CO}$ (and arguably, based on their Figure 9 , it appears worse). Because of the competing effects of photoionization by far-UV 
to produce [C II], and combination with oxygen to produce $\mathrm{CO}$, [C I] is usually not a large reservoir of carbon in the gas, and so its abundance may be subjected to large system-to-system variations depending on the physical conditions.

Adopting an excitation temperature $T_{\mathrm{ex}}=30 \mathrm{~K}$ and a neutral carbon abundance $X_{\mathrm{C}}=(3.0 \pm 1.5) \times 10^{-5}$, appropriate for (U)LIRGs (Weiß et al. 2003, 2005a; Papadopoulos et al. 2004; Walter et al. 2011; Jiao et al. 2017), Cicone et al. (2018b) suggests $\alpha_{[\mathrm{CI}]}=9.43 M_{\odot}\left(\mathrm{K} \mathrm{km} \mathrm{s}^{-1} \mathrm{pc}^{2}\right)^{-1}$ as the [C I](1-0)-to- $\mathrm{H}_{2}$ mass-to-light ratio. Crocker et al. (2019), on the other hand, report $\alpha_{[\mathrm{CI}]}=7.3 M_{\odot}\left(\mathrm{K} \mathrm{km} \mathrm{s}^{-1} \mathrm{pc}^{2}\right)^{-1}$ in a sample of local star-forming galaxy disks targeted by the Herschel Beyond the Peak program.

- Using [C II] Emission to Estimate Outflow Mass. The [C II] $157.737 \mu \mathrm{m}$ finestructure transition is the major cooling transition for the cold atomic medium, at densities $n \sim 1-10^{3} \mathrm{~cm}^{-3}$ and temperatures $\mathrm{T} \gtrsim 50 \mathrm{~K}$. With an ionization potential of $11.3 \mathrm{eV}$, smaller than that of hydrogen, ionization of neutral carbon into $\mathrm{C}^{+}$by far-ultraviolet photons is pervasive. In fact, $\mathrm{C}^{+}$is the major form of carbon in regions illuminated by stars with $A_{V}<1-2$. In molecular regions, far-ultraviolet photons that penetrate the surfaces of interstellar molecular clouds at $A_{V}<1-3$ mag create the socalled photon-dominated or photodissociation regions (PDRs, e.g., Hollenbach and Tielens 1999; Kaufman et al. 1999; Wolfire et al. 1995, 2003), where photoelectric heating effect on dust grains heats the gas and [C II] emission cools it. Some fraction of [C II] is also generated in neutral atomic and in ionized gas, for example in H II regions themselves, where collisions with electrons excite the emission.

Far-infrared [C II] emission is excited by collisions. The collisional partners can be $\mathrm{H}, \mathrm{H}_{2}$, or electrons depending on their abundance in the type of gas the [C II] ion is embedded in - atomic, molecular, or ionized. Because the energy carried away by the $157.7 \mu \mathrm{m}$ photons is significant and the excitation requirements for the transition are not particularly stringent, wherever [C II] is abundant it tends to dominate the cooling. The critical densities for [C II] collisions with $\mathrm{H}, \mathrm{H}_{2}$, and electrons are respectively $\mathrm{n}_{c r}=3000,6100$, and $44 \mathrm{~cm}^{-3}$ taking as typical temperatures $100 \mathrm{~K}$ for atomic clouds and PDRs and 8,000 K for ionized regions (Goldsmith et al. 2012). [CII] emission is usually not significantly optically thick, although in photodissociation regions $\tau$ can be moderate (Boreiko and Betz 1996; Stacey et al. 1991), so optical depth effects are unlikely to have a significant impact on these critical densities.

In the case of optically thin emission and neglibible background, the intensity of the [C II] emission ( $\mathrm{I}_{[\mathrm{CII}}$, in $\mathrm{erg} \mathrm{s}^{-1} \mathrm{~cm}^{-2} \mathrm{sr}^{-1}$ ) relates to the temperature (T), density $(n)$, and column density $(N)$ of the gas through (Crawford et al. 1985; Goldsmith et al. 2012)

$$
\mathrm{I}_{[\mathrm{C} \mathrm{II}]}=2.38 \times 10^{-21}\left[\frac{2 \mathrm{e}^{-91.25 / \mathrm{T}}}{1+2 \mathrm{e}^{-91.25 / \mathrm{T}}+\mathrm{n}_{\text {cr }} / \mathrm{n}}\right] \mathrm{N}\left(\mathrm{C}^{+}\right) .
$$

This can be converted to a relation between luminosity and mass using (see also Hailey-Dunsheath et al. 2010)

$$
M_{\mathrm{H}}=0.68 M_{\odot}\left(\frac{1.6 \times 10^{-4}}{X\left(\mathrm{C}^{+}\right)}\right)\left[\frac{1+2 e^{-91.25 / T}+n_{c r} / n}{2 e^{-91.25 / T}}\right] \frac{L_{[\mathrm{CII}]}}{L_{\odot}},
$$


where $X\left(\mathrm{C}^{+}\right)$is the abundance of $\mathrm{C}^{+}$, and $1.6 \times 10^{-4}$ represents an approximate gasphase carbon abundance near the Solar circle in the Milky Way (Sofia et al. 2004), although the precise value is somewhat uncertain (e.g., Sofia et al. 2011). In the limit of $T \gg 91 \mathrm{~K}$ and $n \gg n_{c r}$ (i.e., the high excitation limit) this becomes

$$
\frac{M_{\mathrm{H}}}{M_{\odot}} \approx\left(\frac{1.6 \times 10^{-4}}{X\left(\mathrm{C}^{+}\right)}\right) \frac{L_{[\mathrm{CII}]}}{L_{\odot}} .
$$

Because we are assuming maximal excitation, this is effectively a lower limit to the amount of mass traced by the [CII] emission. However, if the main contributor to the emission is $\mathrm{C}^{+}$embedded in ionized gas due to HII regions, where dust may not be an important reservoir of carbon, all carbon will be in the gas-phase and its abundance may be a factor of 3 larger (Sofia et al. 2011). Conversely, if the radiation field is such that most carbon is in a more highly ionized state, the $X\left(\mathrm{C}^{+}\right)$would be lower and the $[\mathrm{CII}]$ emissivity per unit gas mass will be suppressed (e.g., Langer and Pineda 2015). Using the relation between integrated Rayleigh-Jeans brightness temperature and intensity for this transition, $\int T_{B} d v=1.43 \times 10^{5} I_{[\mathrm{CII}]}$ (e.g., Goldsmith et al. 2012), we can express this in a form analogous to Eq. (48), keeping in mind that the left hand side represents all hydrogen (not just molecular gas), and that this does not include the 1.36 correction factor by mass due to the contribution from $\mathrm{He}$ and other heavy elements

$$
M_{\mathrm{H}}=0.15 \frac{\mathrm{M}_{\odot}}{\mathrm{K} \mathrm{km} \mathrm{s}^{-1} \mathrm{pc}^{2}}\left(\frac{1.6 \times 10^{-4}}{X\left(\mathrm{C}^{+}\right)}\right) L_{[\mathrm{CII}]}^{\prime} .
$$

Note that this is a lower limit not just because of the assumed excitation and the contribution from moderate optical depth regions, but also because extinction limits the penetration of carbon-ionizing photons, sharply limiting the ionization of carbon inside molecular clouds. For $A_{V}>2-3$ most carbon will be neutral or molecular, and therefore that gas will not emit in [CII] (e.g., Narayanan and Krumholz 2017).

- Using OH Absorption to Estimate Outflow Mass and Spatial Extent. The $\mathrm{OH}$ electronic ground-state ${ }^{2} \Pi$ is split due to spin-orbit coupling where spin angular momentum can be oriented either parallel or anti-parallel to the orbital angular momentum. Thus, the rotational states are split into two ladders: ${ }^{2} \Pi_{3 / 2}$ and ${ }^{2} \Pi_{1 / 2}$ (Storey et al. 1981 ), where the total angular momentum is given by $J=N \pm 1 / 2$ ( $N$ is the rotational quantum number). Additionally, the orbital angular momentum can have two orientations with respect to the molecular axis, each having a slightly different energy. This $\Lambda$-doubling further splits each $J$ level into two levels of opposite parity. $\mathrm{OH}$ and its isotopologues ${ }^{18} \mathrm{OH}$ and ${ }^{17} \mathrm{OH}$ have been detected in absorption in several infrared-bright galaxies. With many transitions lying at far-IR wavelengths, where the bulk of the luminosity of these galaxies is emitted, $\mathrm{OH}$ is mainly excited through absorption of far-IR photons and selectively traces a region close to the central source of strong far-IR radiation density. $\mathrm{OH}$ doublets with a broad range of lower-level energies, from $E_{\text {low }}=0 \mathrm{~K}(\mathrm{OH} 119,79,53.3$, and $35 \mu \mathrm{m}$ originating from the $\Lambda$-doublet state of the ground ${ }^{2} \Pi_{3 / 2} J=3 / 2$ rotational level) to $E_{\text {low }}=620 \mathrm{~K}(\mathrm{OH} 56$ originating from ${ }^{2} \Pi_{1 / 2} J=7 / 2$ ), have been detected in (U)LIRGs, often showing clear signatures of outflowing gas (prominent blue wings or P-Cygni profiles). The detection of $\mathrm{OH}$ 
79, $\mathrm{OH} 53.3$, and $\mathrm{OH} 35$ implies that $\mathrm{OH} 119$, with a much higher opacity, is optically thick i.e. the continuum optical depth at $100 \mu \mathrm{m}, \tau_{100} \gtrsim 1$, or $N_{H} \gtrsim 10^{24} \mathrm{~cm}^{-2}$. However, the $\mathrm{OH} 119$ absorption feature is not black, so the $\mathrm{OH} 119$ doublet at a given velocity only covers a fraction of the total 119 um continuum. When P-Cygni profiles are observed, the relative strength of the redshifted emission (which is produced in the receding cocoons) and blueshifted absorpion (from the approaching parts along the line of sight to the continuum source) can be used to constrain the importance of extinction of the line-emitting photons arising from the back side of the far-IR source and/or significant departure from spherical symmetry (González-Alfonso et al. 2014).

Since the $\mathrm{OH}$ energy levels are radiatively pumped in the outflows, transitions at different wavelengths and energy levels, in combination with continuum component fits, yield crucial information about the radial location where the lines are formed. This information enables the estimation of the outflow physical parameters using the equations in Sect. 3.2. In practice, the observed $\mathrm{OH}$ profiles are carefully compared with the predictions from radiative-transfer models. The code calculates the line excitation due to the dust emission and collisions with $\mathrm{H}_{2}$, and takes into account opacity effects (i.e. radiative trapping), non-local effects, velocity gradients, extinction by dust, and line overlaps. For simplicity, the outflow is usually modeled as a series of concentric expanding shells around a nuclear continuum source, allowing for each source one to three components with different velocity gradients and distances to the central source. The density profile for each velocity component is determined through mass conservation assuming a constant mass outflow rate. Free parameters are the inner and outer radii, the velocity field of each velocity component, the $\mathrm{OH}$ density at the inner radius, the covering factor of the continuum FIR source, and the solid angle of the outflow. For a given model, the code first calculates the statistical equilibrium populations in all shells that make up the source, and then the emerging line shapes are computed, convolved with the instrumental profile, and compared directly with the observations.

The derived outflow mass directly depends on the assumed value of the $\mathrm{OH}$ abundance relative to $\mathrm{H}$ nuclei, $X_{\mathrm{OH}} \equiv N_{O H} / N_{H}$, which is uncertain. A value of $2.5 \times$ $10^{-6}$ has been used in the models of González-Alfonso et al. (2017b), consistent to within a factor of $\sim 3$ with the value inferred from multi-transition observations of $\mathrm{OH}$ in the Galactic Sgr B2 and Orion KL outflow (Goicoechea and Cernicharo 2002; Goicoechea et al. 2006) and in buried galaxy nuclei (González-Alfonso et al. 2012; Fischer et al. 2014; Falstad et al. 2015), and with chemical models of dense photodissociation regions (the peak value in Sternberg and Dalgarno 1995) and of cosmic-ray- and X-ray-dominated regions (Meijerink et al. 2011). New constraints on the value of $X_{\mathrm{OH}}$ in the outflows have recently been derived using the strength of the optically thin cross-ladder ${ }^{2} \Pi_{3 / 2} \rightarrow{ }^{2} \Pi_{1 / 2} J=3 / 2 \rightarrow 5 / 2 \Lambda$-doublet transitions at 34.60 and $34.63 \mu \mathrm{m}$ as a powerful $\mathrm{OH}$ column density estimator (Stone et al. 2018). The $\mathrm{OH}$ column densities in a number of $\mathrm{OH}$ outflows were compared with the hydrogen column density for a typical optical depth at $35 \mu \mathrm{m}$ of $\sim 0.5$ and Galactic gas-to-dust ratio ( 125 ; see Sect. 5.3 below) to yield an $\mathrm{OH}$-to-H abundance ratio of $X_{\mathrm{OH}}=(1.01 \pm 0.15) \times 10^{-6}$. This abundance ratio is formally a lower limit since (1) some fraction of the $\mathrm{OH}$ molecules are in excited levels, so $N_{\mathrm{OH}}$ derived from $\mathrm{OH} 35$ is a lower limit to the column density of all $\mathrm{OH}$ states, and (2) the covering 
fraction of the $35 \mu \mathrm{m}$ continuum source by the obscuring material may not be $100 \%$, as assumed.

\subsubsection{Importance of Shocks, X-rays, and Cosmic Rays}

Chemistry is an important tracer of the conditions in the outflow and hence of its power source and evolution. Chemistry can for example probe the impact of shocks and irradiation by e.g. cosmic rays (CR) and X-rays. Important shock tracers include $\mathrm{SiO}$, which traces fast shocks and the destruction of grains (Bachiller et al. 1991; García-Burillo et al. 2001). Slower shocks that evaporate the ices (without disrupting the grain core) can be probed by e.g. $\mathrm{H}_{2} \mathrm{O}, \mathrm{CH}_{3} \mathrm{OH}, \mathrm{HNCO}, \mathrm{OH}$ and possibly also HCN (e.g. Ruiz et al. 1992; Cernicharo et al. 2006; Rodríguez-Fernández et al. 2010; Aalto et al. 2012; Meier and Turner 2012; Pellegrini et al. 2013; García-Burillo et al. 2014; Goicoechea et al. 2015; Saito et al. 2017). The high HCN luminosity of the Mrk 231 outflow (Sect. 4.4) is likely due both to a large filling factor of dense $\left(n>10^{4}\right.$ $\left.\mathrm{cm}^{-3}\right)$ molecular gas, as well as an elevated HCN abundance $\left(X(\mathrm{HCN})=10^{-8}\right.$ $10^{6}$, Aalto et al. 2012, 2015a; Lindberg et al. 2016). Chemical differentiation in the outflow between $\mathrm{HCN}, \mathrm{HNC}$ and $\mathrm{HCO}^{+}$can be partially caused by shock chemistry (Lindberg et al. 2016). Suspected shock-enhanced $\mathrm{HCN}, \mathrm{SiO}$ and $\mathrm{CH}_{3} \mathrm{OH}$ is found in the outflow regions of the merger NGC 3256 (Sect. 3.4.6; Harada 2018). The CO line-to-continuum ratio (Meijerink et al. 2013) is also a robust diagnostic of shock excitation, as is elevated [C I] line emission (Pellegrini et al. 2013). Shocks are also traced by near-IR [Fe II] 1.257 and $1.644 \mu \mathrm{m}$ lines and $\mathrm{H}_{2}$ line ratios (see Sec 3.4.7).

Note that high $\mathrm{H}_{2} \mathrm{O}, \mathrm{HCN}$, and $\mathrm{CH}_{3} \mathrm{OH}$ abundances may also reflect a hot-core like chemistry where ice mantles are evaporating in high-column density gas (e.g. Cernicharo et al. 2006; González-Alfonso et al. 2012; Qin et al. 2015). GonzálezAlfonso et al. suggest that such an "undepleted" chemistry may be traced by, for example, studying the $\mathrm{HCN} / \mathrm{H}_{2} \mathrm{O}$ and $\mathrm{HCN} / \mathrm{NH}_{3}$ column density ratios. Methanol $\left(\mathrm{CH}_{3} \mathrm{OH}\right)$ primarily forms on cold $(T<24 \mathrm{~K})$ dust grains (e.g. Soma et al. 2015) and can therefore be used as an important "chemical clock". Once depleted from the dust grains it cannot reform as long as the dust grains remain warm.

Radiative impact on the gas can be traced by radicals such as $\mathrm{CN}$ that thrives in the interface regions between photon dominated region (PDRs), or X-ray dominated regions (XDRs), and dense molecular clouds. Far-UV photons in post-shock gas may also impact the chemistry. $\mathrm{CN}$ has been detected in the double outflows of the merger NGC 3256 (Sakamoto et al. 2014) and more studies are required to understand its origin. Very luminous $\mathrm{CN}$ emission is also detected in the quasar-driven molecular outflow of Mrk 231 (Cicone et al. 2019) where $\mathrm{X}(\mathrm{CN})>\mathrm{X}(\mathrm{HCN})$, suggesting strong radiative impact on the outflowing dense molecular gas. $\mathrm{C}_{2} \mathrm{H}$ has been found in the starburst-driven outflow of Maffei 2 (Meier and Turner 2012) and also in the interface region between the molecular disk and ionized gas outflow of NGC 1068 (GarcíaBurillo et al. 2017). The high fractional $\mathrm{C}_{2} \mathrm{H}$ abundances in this interaction region can be reached at very early times ( $t$ of about $10^{2-3} \mathrm{yr}$ ) in models of UV or X-ray irradiated dense gas.

Molecular ions are important tools to probe the ionization rates of the interstellar medium in XDRs/PDRs or in CR-dominated regions (CRDRs). González-Alfonso 
et al. (2018) suggest that high $\mathrm{OH}^{+} / \mathrm{H}_{2} \mathrm{O}^{+}$and $\mathrm{OH}^{+} / \mathrm{H}_{3} \mathrm{O}^{+}$ratios imply high ionization rates per unit density. X-rays appear to be unable to explain the ionization rates inferred by these elevated abundance ratios, and thus they suggest that low-energy (10-400 MeV) CRs are primarily responsible for the ionization (Sect. 4.4). Ionization fraction can also be probed by the $\mathrm{HOC}^{+} / \mathrm{HCO}^{+}$ratio that can be studied at $\mathrm{mm}$ and submm wavelengths (e.g. Goicoechea et al. 2009; Martín et al. 2009). One of the most important probes of ionization rate in diffuse molecular clouds is the $\mathrm{H}_{3}^{+}$ molecule that can be studied at infrared wavelengths. (e.g. Oka et al. 2005; Indriolo et al. 2007).

The $\mathrm{CH}^{+}$molecule is a very useful tracer of turbulence in interstellar gas (e.g. Falgarone and Puget 1995; Myers et al. 2015). Only few extragalactic studies of $\mathrm{CH}^{+}$have been carried out (e.g. Welty et al. 2014; Muller et al. 2017). One concerns the discovery of large turbulent reservoirs of molecular gas surrounding some high-redshift galaxies through the detection of the $\mathrm{CH}^{+}(\mathrm{J}=1-0)$ transition, both in emission and absorption (Falgarone et al. 2017). Given the high critical density of this transition and the very large width of the emission line (FWZI $>2,500 \mathrm{~km} \mathrm{~s}^{-1}$ ) in these objects, it is likely tracing very dense gas $\left(>10^{5} \mathrm{~cm}^{-3}\right)$ ejected by outflows.

It is important to be aware, however, that to safely apply chemistry as a diagnostic tool usually requires a combination of lines to address the dichotomy between PDR, XDRs, CRDRs - and the chemistry of shocks and mechanical heating (e.g. Kazandjian et al. 2012; Aalto 2015; Viti et al. 2014; Viti 2016; Harada et al. 2018).

Molecular chemistry and abundances are usually probed at the $\mathrm{mm} / \mathrm{submm}$ and far-infrared wavelengths. However, X-ray absorption lines and edges may also be used to identify molecules (Gatuzz et al. 2015). Molecules may also be probed at longer $\mathrm{cm}$ wavelengths-for example maser lines of $\mathrm{OH}$ and $\mathrm{H}_{2} \mathrm{O}$ that can be seen in dense dusty outflows (Turner 1985; Baan et al. 1989). Also thermal absorption/emission lines of e.g. $\mathrm{OH}, \mathrm{H}_{2} \mathrm{CO}, \mathrm{NH}_{3}$ can be detected (e.g. Mangum and Wootten 1993; Salter et al. 2008; Mangum et al. 2013; Falstad et al. 2015), also in outflows (Falstad et al. 2017).

\subsubsection{Compact Obscured Nuclei: CONs}

There is a population of luminous infrared galaxies that have highly obscured nuclei with visible extinction $A_{\mathrm{V}}>1000$ (corresponding to $N\left(\mathrm{H}_{2}\right)>10^{24} \mathrm{~cm}^{-2}$ ). At these high levels of extinction, mid-infrared diagnostic methods suffer from opacity effects and one has to resort to longer wavelength lines and continuum to probe the enshrouded activity. The obscuring columns appear to be warm with temperatures in the range of 100-300 K (Sakamoto et al. 2008; Costagliola and Aalto 2010; Sakamoto et al. 2013; González-Alfonso et al. 2012; Aalto et al. 2015b; Falstad et al. 2015; Scoville et al. 2017; Sakamoto et al. 2017; Barcos-Muñoz et al. 2018; Aalto et al. 2019). These enshrouded nuclei may be previously undetected accreting SMBHs and/or compact and luminous young stellar clusters (possibly also with top-heavy stellar initial mass functions (IMFs; Aalto et al. 2019)). Radiatively excited molecular emission of e.g. $\mathrm{H}_{2} \mathrm{O}$ and $\mathrm{OH}$ (van der Werf et al. 2011; González-Alfonso et al. 2012; Veilleux et al. 2013a; González-Alfonso et al. 2014; Falstad et al. 2015, 2017) are good probes of the nuclei as long as column densities stay below $N\left(\mathrm{H}_{2}\right)=10^{25}$ 
$\mathrm{cm}^{-2}$. However, several enshrouded nuclei have $N\left(\mathrm{H}_{2}\right)>10^{25} \mathrm{~cm}^{-2}$ and in some cases column densities may reach extreme values of $N\left(\mathrm{H}_{2}\right)>10^{26} \mathrm{~cm}^{-2}$ (Scoville et al. 2017; Sakamoto et al. 2017; Barcos-Muñoz et al. 2018; Aalto et al. 2019). These Compact Obscured Nuclei (CONs Aalto 2015) are found in the nuclei with radii $r<10-50 \mathrm{pc}$ and high temperatures $>200 \mathrm{~K}$. In the most extreme cases there may also be issues of photon trapping and self-heating of the gas and dust (e.g. Kaufman et al. 1999; González-Alfonso and Sakamoto 2019).

To probe the most deeply enshrouded CONs one needs to go to $\mathrm{mm}$ and even $\mathrm{cm}$ wavelengths, for example using vibrationally excited lines of HCN (Salter et al. 2008; Sakamoto et al. 2010; Aalto et al. 2015b; Martín et al. 2016; Imanishi et al. 2016; Aalto et al. 2019). The HCN molecule has degenerate bending modes in the IR and may absorb IR-photons to its first vibrational state. There are rotational transitions within the vibrationally excited state that can be observed with e.g. ALMA. The energy above ground of the $v_{2}=1$ state is $T_{E / k}=1050 \mathrm{~K}$ requiring high surfacebrightness mid-IR $\left(T_{\mathrm{B}}(14 \mu \mathrm{m}) \approx 100 \mathrm{~K}\right)$ emission to be excited. CONs are also very rich sources of molecular emission that can be used to diagnose the nuclear activity (Martin et al. 2012; González-Alfonso et al. 2012; Costagliola et al. 2016).

\subsubsection{Direct $\mathrm{H}_{2}$ Probes}

Molecular hydrogen is the most abundant molecule in the Universe, but direct observations of $\mathrm{H}_{2}$ are difficult. The molecule exists in two, almost independent isometric forms, named ortho- $\mathrm{H}_{2}$ (spin of $\mathrm{H}$ nuclei parallel) and para- $\mathrm{H}_{2}$ (spins antiparallel). The ortho/para ratio is a complex function of the environment (e.g., Bron et al. 2016). In LTE, the ortho/para ratio is close to 3 under warm $(T>200 \mathrm{~K})$ conditions, but is generally smaller otherwise. Given its symmetry, $\mathrm{H}_{2}$ has no permanent dipole moment and all its ro-vibrational and rotational transitions within the electronic ground state are forbidden electric quadrupolar and therefore weak. The ro-vibrational lines lie in the near-IR, and thus can be studied from the ground at low redshifts, while the rotational lines occur in the mid-IR and generally require space-borne facilities. The far-UV electronic transitions from nearby systems can only be detected above the Earth's atmosphere and are strongly affected by extinction. Indeed the very presence of $\mathrm{H}_{2}$ requires $A_{V} \gtrsim 0.01-0.1 \mathrm{mag}$. so that it is shielded from the ultraviolet photons responsible for its photo-dissociation.

- Electronic Transitions. Photons with an energy in the range of 11.2 to $13.6 \mathrm{eV}$ (912 $\AA$ to $1126 \AA$ ) that coincides with one of the lines in the Lyman band (between the ground and first electronic states; $B-X$ ) or Werner band (between the ground and second electronic states; $C-X$ ) will be absorbed by $\mathrm{H}_{2}$ and place the molecule in an excited electronic state, followed by rapid radiative decay that may lead to its dissociation. This destruction process is balanced by formation of $\mathrm{H}_{2}$ on the surface of dust grains (Sect. 3.5.6). The Lyman and Werner electronic absorption bands in the far-UV provide a very sensitive tool to detect even very diffuse $\mathrm{H}_{2}$, down to column densities as low as a few $10^{12} \mathrm{~cm}^{-2}$, provided a UV-bright background source is available (e.g. Spitzer and Cochran 1973). A curve-of-growth analysis that relies on the measured equivalent widths of all $\mathrm{H}_{2}$ lines is possible when $N\left(\mathrm{H}_{2}\right) \lesssim 10^{19} \mathrm{~cm}^{-2}$, 
while a fit to the damping wings of the low- $\mathrm{J}_{2}$ lines is needed to derive larger column densities. $\mathrm{H}_{2}$ column densities in low rotational state $(J=0$ and 1$)$ may be used to derive rotational and/or kinetic temperatures of the diffuse gas. The higher $J$ abundances can constrain models of the UV radiation fields and gas densities (e.g. Shull et al. 2000; Wakelam et al. 2017).

- Ro-Vibrational Transitions. In the ground-state electronic state, the $v=1$ vibration level is $\sim 6000 \mathrm{~K}$ above the ground state, so that ro-vibrational excitation requires kinetic temperatures $T>1000 \mathrm{~K}$ or fluorescent ("FUV pumping") excitation. The method most commonly used to differentiate collisional excitation from fluorescence consists in using flux ratios of various ro-vibrational $\mathrm{H}_{2}$ lines visible in the $\mathrm{K}$ band, particularly $\mathrm{H}_{2} v=1-0 \mathrm{~S}(1) 2.121 \mu \mathrm{m}$ and the weaker $v=1-0 \mathrm{~S}(0) 2.223 \mu \mathrm{m}$ and $v=2-1 \mathrm{~S}(1) 2.247 \mu \mathrm{m}$ transitions. FUV pumping dominates if $G_{0} / n$ is large $\left(G_{0}\right.$ is the strength of the UV radiation field and $n$ is the hydrogen density in the PDR). In the alternative scenario of collisional excitation, three mechanisms may provide the heating: (1) UV radiation from a starburst or AGN, (2) X-rays from a starburst, AGN, or wind, or (3) shocks induced by the outflow. A strong constraint on the importance of UV heating can be derived from the ratios of $\mathrm{H}_{2} 2.12 \mu \mathrm{m}$ to $\mathrm{H} \alpha$ (or, equivalently, Br $\gamma$; e.g., Puxley et al. 1990; Doyon et al. 1994), while the relative $\mathrm{H}_{2}$ 1-0 S(1) and $\mathrm{X}$-ray fluxes provide an excellent way to test the mechanism of X-ray heating. The third and final scenario may be tested using shock diagnostics (e.g. [Fe II] $1.644 \mu \mathrm{m}$, mm-wave SiO; see Sec. 3.4.5).

- Pure Rotational Transitions. The $\mathrm{H}_{2}$ pure-rotational emission lines in the mid-IR trace warm molecular gas at $T=100-1000 \mathrm{~K}$. Due to the low Einstein coefficients of these transitions, optical depth effects are usually unimportant and thus these midIR lines provide an accurate measure of the warm gas mass. At densities $n_{H} \gtrsim 10^{4}$ $\mathrm{cm}^{-3}$ of PDRs, collisions maintain the lowest rotational levels of $\mathrm{H}_{2}(v=0, J \lessgtr 5)$ in thermal equilibrium. The rotational transitions between these levels therefore provide a thermometer for the bulk of the gas above $\sim 80 \mathrm{~K}$. The excitation temperatures measured from these line ratios should be considered an upper limit to the kinetic temperature since $\mathrm{UV}$ pumping may contribute to the excitation of $\mathrm{H}_{2}$ even for low $J$. Shocks may also contribute to exciting the $\mathrm{H}_{2}$ molecules without dissociating them. The excitation temperature is a steep function of shock velocity and therefore can be used to constrain it if shocks are dominant (e.g., Guillard et al. 2012; Beirão et al. 2015).

\subsection{Diagnostic Tools of the Dust}

There is extensive literature on this topic including the excellent review of Galliano et al. (2018) and the classic monograph by Draine (2011). The many diagnosic tools that have been used to derive the dust properties in galaxies may also be applied to study the dust within cool outflows. These tools can largely be divided into five main categories based on the physical process at work: (1) extinction and reddening, (2) scattering, (3) emission, (4) polarization, and (5) depletion. The derived properties include the dust composition (the exact mixture of carbonaceous and silicate grains), 
the grain structure (e.g., amorphous, crystalline, porous), the size distribution of the dust grains (typically, sizes $a$ range from $0.3 \mathrm{~nm}$ to $0.3 \mu \mathrm{m}$ with a power-law distribution $\propto a^{-3.5}$ to first order), the abundance of the dust grains relative to the gas (i.e. the dust-to-gas ratio by mass, $D / G$, of order $\sim 1 \%$ in the Milky Way, and a decreasing function of metallicity; Roman-Duval et al. 2014), and the fraction of dust present in the ionized gas ( $f_{\text {ion }}$ ranging from $\sim 10^{-4}$ in the hot intracluster medium of galaxy clusters to $\sim 1$ in some luminous infrared galaxies; Laursen et al. 2009).
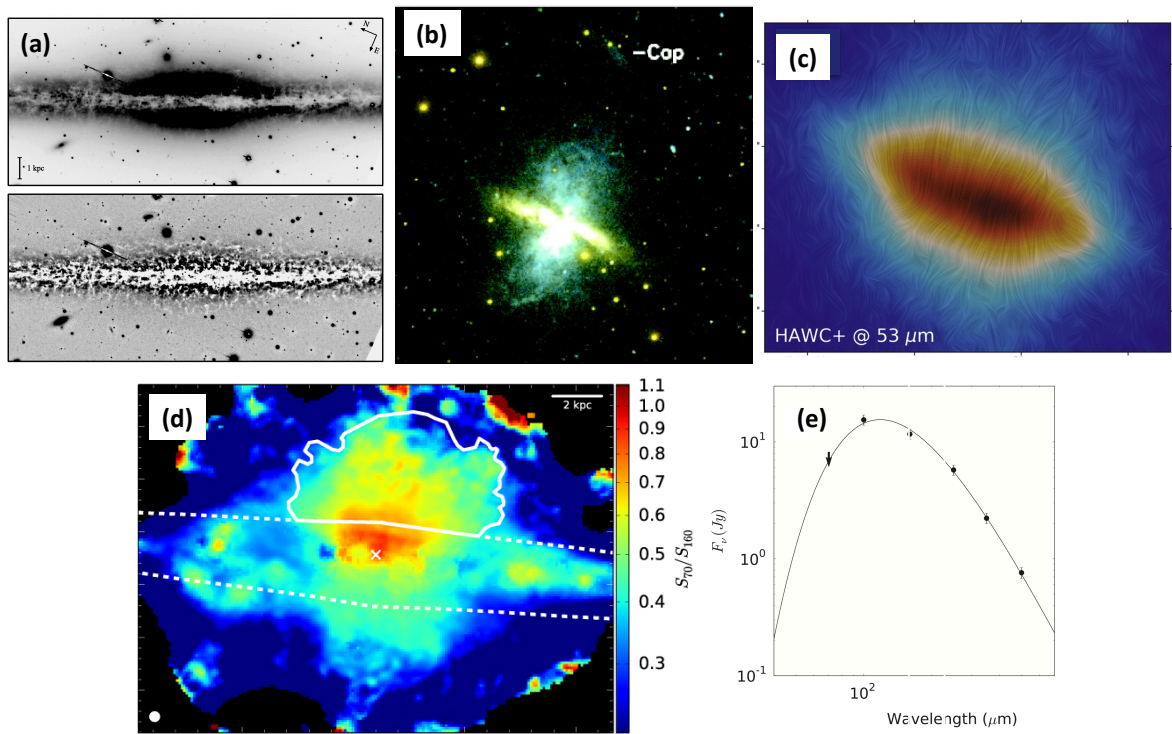

Fig. 8 Some of the diagnostic tools used to study dust in cool outflows. (a) Extinction in the dusty extraplanar material of NGC 891, (b) UV scattering in the dusty wind of M 82, (c) polarization in the disk and wind of M 82, (d)-(e) thermal infrared emission from the extraplanar material in NGC 4631. Images reproduced with permission from (a) Howk and Savage (2000), (b) Hoopes et al. (2005), (c) Jones et al. (2019b), and (d, e) Meléndez et al. (2015), copyright by AAS.

\subsubsection{Extinction and Reddening}

The optical depth due to dust is $\tau_{d}=\int \sigma_{d} n_{H} d s \simeq \sigma_{d} N_{H}$, where $\sigma_{d}$ is defined as the effective cross section per hydrogen atom. This term includes contributions from both absorption and scattering: $\sigma_{d}=\sigma_{a}+\sigma_{s}$. A related quantity is the dust opacity, $\kappa_{d}=\kappa_{a}+\kappa_{s}$, which is the effective cross section per dust mass so that $\rho \kappa_{d}=n_{H} \sigma_{d}$. The observed extinction $A_{\lambda}$ in magnitudes is directly related to $\sigma_{d}$ and scales linearly with $N_{H}$ :

$$
A_{\lambda}=\frac{2.5}{\ln 10} \tau_{d}=1.086 \sigma_{d} N_{H}
$$

Both absorption and scattering increase at shorter wavelengths causing reddening of background objects. The slope of the extinction curve in the optical region is often 
measured by the dimensionless quantity

$$
R_{V} \equiv A_{V} /\left(A_{B}-A_{v}\right)=A_{V} / E(B-V),
$$

where $\mathrm{B}$ and $\mathrm{V}$ refer to the $\mathrm{B}$ and $\mathrm{V}$ bands and $E(B-V)$ is the color excess. This quantity ranges from $R_{V} \simeq 1.2$, for very small grains (grain size $a<<\lambda$ ) where Rayleigh scattering $\left(A_{\lambda} \propto \lambda^{-4}\right)$ is dominant, up to $R_{V} \rightarrow \infty$ for grey extinction from very large grains $(a>>\lambda)$. The "classical" value, $R_{V}=3.1$, applies to the diffuse ISM in the Milky Way (in that case, $A_{V} \simeq 0.53\left(N_{\mathrm{H}} / 10^{21} \mathrm{~cm}^{-2}\right)$ mag; Cardelli et al. 1989), but in practice $R_{V}$ shows significant scatter $\left(R_{V} \approx 2-6\right)$ within the Milky Way and in external galaxies, which underscores the environmental dependence of extinction.

The overall $0.1-30 \mu \mathrm{m}$ extinction curve is characterized by a number of features (see Fig. 6 in Galliano et al. 2018): (a) a steep rise in the FUV (sometimes as steep as $\lambda^{-1}$ ), primarily attributed to absorption by small dust grains, (b) a bump around 2175 $\AA$ bump, produced by small carbon grains such as PAHs, graphite, or amorphous carbon, (c) a knee around $\sim 4000 \AA$ due to scattering by large grains, and (d) a powerlaw optical-NIR continuum (roughly $\propto \lambda^{-1}$ ). In addition, bright infrared sources with $A_{18 \mu \mathrm{m}} \gtrsim 1 \mathrm{mag}$. ( $A_{V} \gtrsim 12 \mathrm{mag}$.) often present two prominent absorption features at 9.7 and $18 \mu \mathrm{m}$, caused mainly by amorphous silicate although the distinct absorption features of unprocessed crystaline silicate have been detected in some ULIRGs (Spoon et al. 2006). The infrared spectra of the most obscured sources also present $\mathrm{H}_{2} \mathrm{O}, \mathrm{CO}$, and $\mathrm{CO}_{2}$ ice absorption features at 3.1, 4.7, and $15.2 \mu \mathrm{m}$, produced by icy grain mantles deeply shielded from heating sources.

\subsubsection{Scattering}

The albedo, $A_{s, \lambda} \equiv \sigma_{s} / \sigma_{d} \leq 1$, is a measure of the importance of scattering on the overall extinction. For instance, in the NUV, $A_{s, \lambda} \simeq 0.5$ (Draine 2003). For a Galactic dust-to-gas ratio, $D / G \approx 0.01$, the dust scattering cross-section in the UV-optical is $>1-2$ orders of magnitude larger than the Thompson scattering cross-section of electrons $\left(\sigma_{T}=6.65 \times 10^{-25} \mathrm{~cm}^{-2}\right)$. The dust scattering cross-section and its phase function (angular dependence) may be decomposed into two functions (Weingartner and Draine 2001; Draine 2003):

$$
\left(\frac{d \sigma_{s}}{d \Omega}\right)=C_{s}(\lambda) \times p(\theta),
$$

where $\theta$ is the scattering angle between the observer's line of sight and the initial direction of propagation of the photon from the light source to the scattering event. For simplicity, $C_{s}$ may be approximated as a power-law with wavelength $C_{s}(\lambda) \propto \lambda^{-\alpha}$, where the index $\alpha$ depends on $x \equiv(2 \pi a) / \lambda$, ranging from $\alpha \rightarrow 4$ in the Rayleigh scattering limit $(x<<1)$ to $\alpha \rightarrow 0$ in the geometric optics limit $(x>>1)$. If all dust grains had the same size, one would therefore expect a break in the wavelength dependence of the scattered light at a critical wavelength $\lambda_{c} \simeq a$. In reality, the grain sizes show a broad distribution which smears the spectral break.

In the weak scattering approximation (i.e. each photon scatters at most once), and assuming the energy source is a point source of luminosity $L_{\lambda}$, the radiative transfer 
equation of photons through a scattering screen is

$$
\frac{d I_{s, \lambda}}{d s} \simeq\left(\frac{\Phi_{\lambda}}{4 \pi}\right)\left(\frac{L_{\lambda}}{4 \pi r^{2}}\right) n_{H} \sigma_{s, \lambda}
$$

where $s$ is the path length, $I_{s, \lambda}$ is the measured scattered intensity, $L_{\lambda} /\left(4 \pi r^{2}\right)$ is the incident intensity at the scattering location, and $\Phi_{\lambda}$ is a wavelength-dependent factor that captures the anisotropy of dust scattering. In the idealized case of a spherically symmetric scattering screen surrounding a point source (e.g., dusty halo around an AGN or compact starburst), the integrated scattered luminosity is simply

$$
L_{s, \lambda} \simeq L_{\lambda}\left(1-e^{-\tau_{s, \lambda}}\right) \simeq L_{\lambda} \tau_{s, \lambda},
$$

where a scattering optical depth $\tau_{s, \lambda} \equiv(D / G) N_{\mathrm{H}} \sigma_{s, \lambda}<<1$ was assumed. While this expression can be used in principle to estimate the dust column densities $(D / G) N_{\mathrm{H}}$ of the scattering screen, a more accurate measurement requires using a radiative transfer scattering model where a geometric model for the halo dust and disk emission is adopted (Sect. 5.3).

\subsubsection{Emission}

Dust grains are heated by the absorbed photons and this energy is re-emitted at longer wavelengths as infrared radiation. The dust mass may be estimated to first order by fitting a simple single-temperature modified black body (MBB) to the infrared spectral energy distribution using

$$
F_{v}=\frac{M_{d} K_{v} B_{v}\left(T_{d}\right)}{d^{2}}
$$

Here $M_{d}$ is the dust mass (free parameter), $B_{v}$ is the Planck function, $T_{d}$ is the dust temperature (free parameter), $d$ is the distance to the galaxy, and $\kappa_{v}$ is the dust emissivity, $\kappa_{v}=\kappa_{0}\left(v / v_{0}\right)^{\beta}$, where $\kappa_{0}$ is the dust opacity at $350 \mu \mathrm{m}$. A commonly used value for $\kappa_{0}$ is $0.192 \mathrm{~m}^{2} \mathrm{~kg}^{-1}$; this value of the dust opacity is based on the best fit of the average FIR dust emissivity for the Milky Way model presented in Draine (2003), which yields a best-fit spectral index value of $\beta=2.0$. Caution must be taken as the normalized dust model opacity cross-section, $\kappa_{0}$, is associated with a dust model with $\beta=2.0$ and thus, discrepancies may arise between the results from a singletemperature MBB fit with a fixed emissivity and with an emissivity as a free parameter (see Bianchi 2013, for a review). Note also that more recent fits to the infrared SED of the Milky Way, and other Local Group galaxies, suggest a lower value for $\beta$ (= 1.62; e.g., Planck Collaboration et al. 2014). These fits may be refined by adding additional MBB components with different dust temperatures to better capture the full infrared SED. These simple MBB fits are usually carried out using the infrared SED above $\sim 30 \mu \mathrm{m}$, which is largely featureless and dominates the dust mass determinations. The far-infrared and submm-wave emissivity increases with the porosity of dust grains (Jones 1988).

The underlying assumption of this fitting method is that the dust is in thermal equilibrium wih the ambient radiation field. This is true for large dust grains with 
large enthalpy, but that is not the case for small grains $(a \lesssim 0.02 \mu \mathrm{m})$ where the absorption of a single photon will cause a significant temperature spike ("stochastic" heating). These temperature fluctuations complicate the small-grain dust mass estimates, although constraints from Spitzer spectra have helped refine the absorption properties of these small grains and thereby their contributions to the overall dust masses (e.g., Draine et al. 2007; Chastenet et al. 2019). The small grains that emit in the mid-infrared have characteristic broad emission features with Drude-like profiles centered at 3.3, 6.2, 7.7, 8.6, 11.2, 12.7, and $17 \mu \mathrm{m}$. These features are most likely due to optically active vibrational modes of $\mathrm{C}-\mathrm{C}, \mathrm{C}-\mathrm{C}-\mathrm{C}$, and $\mathrm{C}-\mathrm{H}$ bonds in polycyclic aromatic hydrocarbons (PAHs) with $\lesssim 10^{3}$ carbon atoms. The relative intensity of these features may be used to diagnose two key properties of the PAH population: brighter short-wavelength PAH features relative to the long-wavelength features indicates an excess of small PAHs in the PAH population, while bright 6-9 $\mu \mathrm{m}$ features indicate more abundant $\mathrm{PAH}^{+}$ions relative to $\mathrm{PAH}^{0}$ molecules (e.g., Draine and $\mathrm{Li}$ 2007; Beirão et al. 2015).

Two broad silicate features from warm $(\sim 200 \mathrm{~K})$ dust have been detected in emission at $10 \mu \mathrm{m}$ and $18 \mu \mathrm{m}$ in several type 1 and 2 quasars and AGN, including a low-luminosity LINER (e.g., Siebenmorgen et al. 2005; Hao et al. 2005; Sturm et al. 2005, 2006; Teplitz et al. 2006; Schweitzer et al. 2008). The strength of the $10 \mu \mathrm{m}$ bump relative to that of the $18 \mu \mathrm{m}$ bump is a diagnostic of the dust temperature and can thus be used to estimate the location of the warm dust with respect to the energy source as long as the incident heating spectrum and intensity are known. For instance, Schweitzer et al. (2008) have argued that the bulk of the silicate emission in type 1 PG QSOs arises from dust in the innermost portion of the NLR $(\sim 10-300 \mathrm{pc})$, well outside of the dust sublimation radius (Sec. 3.5.6).

Finally, dust continuum emission has also been detected below $\sim 10 \mu \mathrm{m}$ in the Milky Way and external galaxies (e.g., the "extended near-infrared emission" at 3-7 $\mu \mathrm{m}$ in Sellgren et al. 1983; Barvainis 1987; Sturm et al. 2000; Xie et al. 2018). In general, grains heated by single photons of energy $h v$ radiate most of their energy at $T_{\text {peak }}$ given by $T_{\text {peak }} \propto a^{-3 / 4}(h v)^{1 / 4}$. The dust emission below $10 \mu \mathrm{m}$ therefore indicates the presence of very small grains (VSGs) and nanometer-size particles at $T=300-1500 \mathrm{~K}$.

\subsubsection{Polarization}

Dust may induce polarization through scattering, dichroic extinction, and dichroic emission. The level of linear polarization due to dust scattering reflects the dust composition, grain size, and spatial distribution with respect to the light. It peaks in the FUV where $\sigma_{s}$ is largest and strongly forward directed (e.g., Zubko and Laor 2000). Dichroic extinction is produced when photons propagate through a screen of dust where the elongated grains are aligned along the magnetic field. The polarization is perpendicular to the magnetic field and peaks in the optical according to $P(\lambda)=P_{\max } \exp \left[-0.92 \ln ^{2}\left(\lambda_{\max } / \lambda\right)\right]$, where $\lambda_{\max }=0.55 \mu \mathrm{m}$ (Serkowski et al. 1975; Draine and Allaf-Akbari 2006), followed by a power-law extension from $1.4 \mu \mathrm{m}$ up to $4 \mu \mathrm{m}$ with an index of -1.7 (Martin et al. 1992; Draine and Fraisse 2009). The warm dust in this screen will also produce polarized thermal emission, but in this 
case the polarization is parallel to the magnetic field. Spectropolarimetry in the UVoptical has also been used to measure the velocity of the dusty scatterers relative to that of the line-emitting gas (e.g., Yoshida et al. 2011, 2019).

\subsubsection{Depletion}

Dust primarily consists of silicates $(\mathrm{Mg}, \mathrm{Fe}, \mathrm{Si}, \mathrm{O}, \mathrm{Na}$, and $\mathrm{Al}$ in various combination including $\mathrm{Mg}_{2} \mathrm{SiO}_{4}, \mathrm{MgFeSiO}_{4}, \mathrm{MgSiO}_{3}, \mathrm{Mg}_{0.5} \mathrm{Fe}_{0.5} \mathrm{SiO}_{3}, \mathrm{Na}_{0.5} \mathrm{Al}_{0.5} \mathrm{SiO}_{3}, \mathrm{SiO}_{2}$, and $\mathrm{SiC}$; e.g., Bouwman et al. 2001; Min et al. 2007) and carbonaceous material (C in graphite and organics). Refractory elements with high condensation temperatures (e.g., many of the iron peak elements primarily produced in Type Ia $\mathrm{SNe}$ ) are overrepresented in dust grains compared with volatile elements (e.g., elements arising from $\alpha$-capture processes), resulting in differential elemental depletion in the gas phase when dust is present. This depletion pattern has been used to constrain the dust content of a wide variety of environments in the Milky Way and elsewhere (e.g., Savage and Sembach 1996; Jenkins 2009). Depletion is seen to scale with the mean density of the medium (e.g., Savage and Bohlin 1979; Crinklaw et al. 1994). Following Jenkins (2009) and Galliano et al. (2018), the depletion of an element X is defined as

$$
\left[\frac{X_{\mathrm{gas}}}{H}\right] \equiv \log \left(\frac{X}{H}\right)_{\mathrm{gas}}-\log \left(\frac{X}{H}\right)_{\mathrm{ref}} \simeq\left[\frac{X_{\mathrm{gas}}}{H}\right]_{0}+A_{X} \times F_{*}
$$

where the term with subscript "ref" is the reference value of the abundance when no dust is present. The depletion is approximated as a first-order polynomial with a fixed term, the minimum depletion, which corresponds to the core of the grains, and a varying environmental factor attributed to accretion of mantles in denser environments ( $F_{*}$ is called the depletion strength). While most depletion measurements in the neutral and ionized phases of our Milky Way and external galaxies have been made using UV-optical absorption lines, X-ray spectroscopy may be used to determine the depletion pattern in hot plasmas by measuring the depth, structure, and energy of the photoelectric absorption edges of $\mathrm{C}(\mathrm{K}, 284 \mathrm{eV}), \mathrm{O}(\mathrm{K}, 538 \mathrm{eV}), \mathrm{Fe}\left(\mathrm{L}_{2,3}, 708,721\right.$ eV) $\mathrm{Mg}(\mathrm{K}, 1311 \mathrm{eV}), \mathrm{Si}(\mathrm{K}, 1846 \mathrm{eV})$, and Fe (K, $7123 \mathrm{eV})$ (e.g., Schulz et al. 2002; Draine 2003; Gatuzz et al. 2015, 2016).

\subsubsection{Dust Cycle: Formation and Destruction}

Dust is created in the cooling ejecta of asymptotic giant branch (AGB) stars with

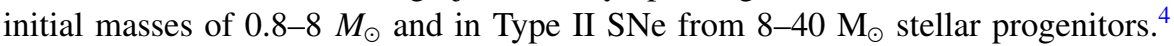
The detection of dust at $z>6$ implies a significant contribution from supernovae in the early universe (Maiolino et al. 2004). The dust grains produced by AGBs and SNe act as seeds that grow, via accretion, in the ISM, on a time scale (Asano et al. 2013)

$$
t_{\mathrm{acc}} \simeq 2.2 \times 10^{4}\left(\frac{a}{0.1 \mu \mathrm{m}}\right)\left(\frac{n_{H}}{10^{4} \mathrm{~cm}^{-3}}\right)^{-1}\left(\frac{T}{10^{4} K}\right)^{-1 / 2}\left(\frac{Z}{0.0129}\right)^{-1} \mathrm{yr} .
$$

\footnotetext{
4 Sarangi et al. (2019) have recently suggested that nuclear AGN winds may also be sites of dust formation.
} 
Several processes may destroy this dust including collisions with other grains, sputtering due to collisions with ions, sublimation or evaporation, explosion due to ultraviolet radiation, and alteration of grain material by cosmic rays and X-rays. Many of these processes are associated with SN shocks. In this context, a distinction is made between thermal sputtering, where the sputtering rate only depends on the local gas properties (namely $n_{H}$ and $T$ ), and non-thermal (inertial) sputtering, where the dustgas relative velocity is a crucial parameter. The thermal sputtering timescale is

$$
t_{\mathrm{sput}} \simeq 3.3 \times 10^{3}\left(\frac{a}{0.1 \mu \mathrm{m}}\right)\left(\frac{n_{H}}{10 \mathrm{~cm}^{-3}}\right)^{-1}\left(\frac{Y_{\mathrm{tot}}}{10^{-6} \mu \mathrm{m} \mathrm{yr}^{-1} \mathrm{~cm}^{3}}\right)^{-1} \mathrm{yr},
$$

where $Y_{\text {tot }}$ is the sputtering yield. For both silicate and carbonaceous dust, the sputtering yield hovers around $\sim 10^{-6}$ for $10^{6} \mathrm{~K} \lesssim T \lesssim 10^{9} \mathrm{~K}$, and drops precipitously below $\sim 10^{6} \mathrm{~K}$ (Nozawa et al. 2006; Hu et al. 2019).

This timescale is shortened by factors of $\sim 2-3$ in SN environments where nonthermal sputtering is important (e.g., Hu et al. 2019). The threshold temperature below which sputtering becomes inefficient corresponds to a shock velocity $v_{s} \simeq$ $300 \mathrm{~km} \mathrm{~s}^{-1}\left(T_{s} / 10^{6} \mathrm{~K}\right)^{1 / 2}$. It is not clear at present to what extent dust is able to survive faster shocks (e.g., Arendt et al. 2010; Lakićević et al. 2015; Temim et al. 2015; Dopita et al. 2016), although the presence of dust behind the reverse shock in some SN remnants (e.g., Kochanek 2011; Matsuura et al. 2019) and in galaxies in general (Gall and Hjorth 2018) suggests that dust is either more resilient than originally predicted (e.g., Silvia et al. 2010; Biscaro and Cherchneff 2016) or it reforms rapidly behind the shocks (Humphreys et al. 2012; Seale et al. 2012; Gall et al. 2014) despite models predicting the contrary (e.g., Biscaro and Cherchneff 2014).

In a galaxy with a powerful nuclear source of UV radiation like an AGN, dust sublimation will also be a factor. Dust will sublimate if located within

$$
r_{\text {subl }}=1.3\left(\frac{L_{\mathrm{UV}}}{10^{46} \mathrm{erg} \mathrm{s}^{-1}}\right)^{1 / 2}\left(\frac{T}{1500 K}\right)^{-2.8} \mathrm{pc}
$$

from the AGN (Barvainis 1987; Netzer 2013). This expression assumes graphite grains with $a=0.05 \mu \mathrm{m}$ and an evaporation temperature of $\sim 1500 \mathrm{~K}$. Silicate grains have a lower evaporation temperature of $\sim 800-1500 \mathrm{~K}$, depending on the grain size and exact composition, resulting in a larger sublimation radius (Kimura et al. 2002).

\section{Best-Studied Cases of Cool Outflows}

4.1 The Milky Way and the Magellanic Clouds

\subsubsection{The Milky Way}

The Milky Way is not only the nearest laboratory at our disposal to study cool outflows, it is also an excellent test case for quenched galaxies since it lies squarely in the "green valley" (Mutch et al. 2011; Bland-Hawthorn and Gerhard 2016) and has not undergone any significant galaxy mergers in the past $\sim 8-11$ Gyr (e.g., Belokurov 
et al. 2018) that would have erased the signatures of fossil outflows or revived the star formation activity by bringing new fuel for the next generation of stars.

The existence of a large-scale outflow centered on the Galaxy center (GC; $d=$ $R_{0}=8.18 \mathrm{kpc}, 1 \mathrm{pc}=25.2^{\prime \prime}$; Gravity Collaboration et al. 2019) has been suspected for many years. Long before the Fermi discovery of two giant $\sim 12-\mathrm{kpc} 1-100 \mathrm{GeV}$ $\gamma$-ray emitting bubbles extending $\sim 55^{\circ}$ on either side of the Galactic center (Fig. 9a; Su et al. 2010; Dobler et al. 2010; Ackermann et al. 2014) and the earlier discovery of a microwave "haze" in the data from the Wilkinson Microwave Anisotropy Probe (WMAP; Finkbeiner 2004; Dobler and Finkbeiner 2008; Planck Collaboration et al. 2013), it has been argued that the large radio-emitting shells and filaments seen in the direction of the GC (particularly the $\Omega$-shaped Galactic Center Lobe reported by Sofue and Handa 1984) and their X-ray counterparts in the 1.5-keV ROSAT all-sky maps (Fig. 9d; Bland-Hawthorn and Cohen 2003) are indicative of a large ( $\pm 3 \mathrm{kpc}$ ) bipolar outflow of warm-hot plasma centered on the GC. Recently, it has been shown that the Galactic Center Lobe is part of a larger bipolar radio structure spanning 430 pc centered on the GC (Fig. 9c; Heywood et al. 2019). The X-ray emission in the central $\pm 2^{\circ}( \pm 250 \mathrm{pc})$ has also recently been mapped in exquisite details with XMMNewton and Chandra (Fig. 9b; Ponti et al. 2019) (see also Nakashima et al. 2019). Two bipolar lobes are observed, one reaching the base of the northern Fermi Bubble $\sim 160 \mathrm{pc}$ from the GC and the other extending beyond the base of the southern Fermi bubble out to $\sim 250 \mathrm{pc}$ (Fig. 9b). The thermal energy of these X-ray structures is modest, $\sim 4 \times 10^{52} \mathrm{ergs}$, corresponding to a power of $\sim 4 \times 10^{39} \mathrm{erg} \mathrm{s}^{-1}$ for a dynamical timescale of $\sim 3 \times 10^{4} \mathrm{yr}$ (Ponti et al. 2019). Similar numbers are derived from the radio structures (Heywood et al. 2019). This power can in principle be provided by supernovae in the central star cluster ( $\mathrm{Lu}$ et al. 2013) or a minor accretion or stellar tidal disruption event (Rees 1988$)$ onto the SMBH in the GC $\left(M_{\mathrm{BH}}=4.15 \times 10^{6} \mathrm{M}_{\odot}\right.$; Gravity Collaboration et al. 2019).

In contrast, the power needed to create the Fermi Bubbles is several orders of magnitude larger, of order $10^{42-44} \mathrm{erg} \mathrm{s}^{-1}$ (or $10^{56}-10^{57}$ ergs in total), depending on the exact duration of the activity and how long ago this energy was injected in the GC. These numbers favor a Seyfert-like SMBH accretion event in the recent past (a few Myr ago) rather than a starburst event as the energy source for the Fermi Bubbles and related structures (e.g. Zubovas et al. 2011; Guo and Mathews 2012; Yang et al. 2012; Bland-Hawthorn et al. 2013; Mou et al. 2014; Ruszkowski et al. 2014; Miller and Bregman 2016; Bland-Hawthorn et al. 2019). ${ }^{5}$ The scenario in which slow but sustained $\left(\approx 10^{8}-10^{9} \mathrm{yr}\right.$ ) energy injection from protracted star formation activity in the GC is responsible for the Fermi Bubbles (e.g., Crocker and Aharonian 2011; Crocker et al. 2015) appears to be inconsistent with the energy injection rate inferred from the X-ray properties of the MW hot halo $\left(\sim 2 \times 10^{42} \mathrm{erg} \mathrm{s}^{-1}\right.$, Miller and Bregman 2016 ) and the spatially resolved stellar population within the inner $\sim 500 \mathrm{pc}$ of the GC (time-averaged star formation rate $\dot{M}_{*} \lesssim 0.1 M_{\odot} \mathrm{yr}^{-1}$ over the last $10^{6}-10^{10} \mathrm{yr}$, implying an energy injection rate $\lesssim 10^{40} \mathrm{erg} \mathrm{s}^{-1}$; e.g., Yusef-Zadeh et al. 2009; Bland-

\footnotetext{
5 There is also evidence that the AGN in the GC was $\sim 10^{5}$ more active in the recent past $\left(\sim 10^{2-3} \mathrm{yrs}\right)$, although still greatly sub-Eddington $\left(L / L_{\mathrm{Edd}} \lesssim 10^{-5}\right)$, based on the detection of strong fluorescent $\mathrm{Fe} \mathrm{K} \alpha$ line emission off of molecular clouds near the GC (Sunyaev et al. 1993; Koyama et al. 1996; Ponti et al. 2010).
} 
Hawthorn et al. 2013; Barnes et al. 2017). While the exact value of this average star formation rate is still a matter of debate, ${ }^{6}$ all estimates fall short by at least an order of magnitude to explain the energy injection rate inferred from the MW X-ray data.

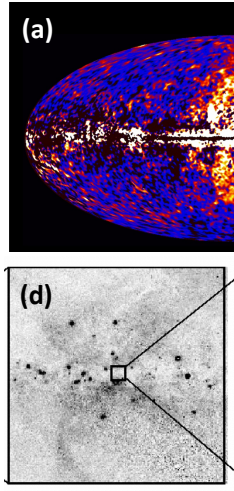

$44^{\circ} \times 4^{\circ}$

(e)

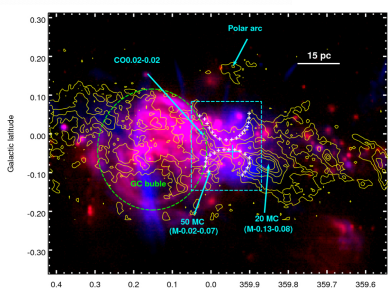

(g)

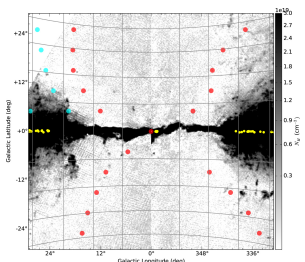

(b)

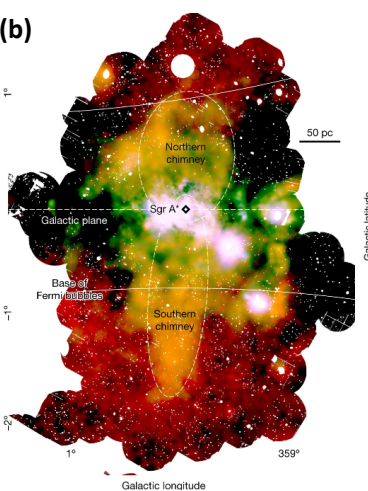

(c)

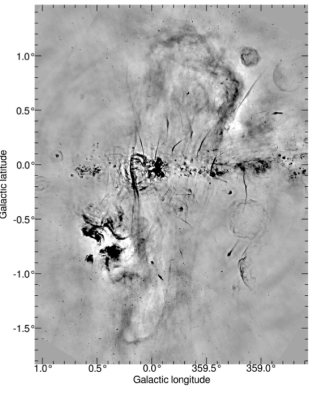

(f)

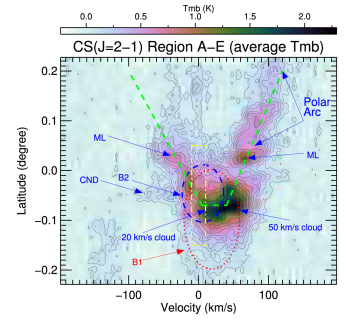

(h)

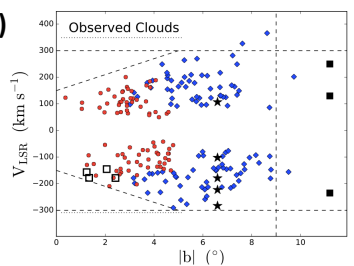

Fig. 9 The multi-phase outflow of the Milky Way. (a) All-sky gamma-ray emission map showing the \pm $55^{\circ}$ Fermi Bubbles. (b) X-ray lobes extending ${ }_{-1.5}^{+1.0}$ degrees from the Galactic plane. (c) Radio structure approximately on the same scale as in panel (b). (d) X-shaped $1.5 \mathrm{keV}$ emission (left; $44^{\circ} \times 44^{\circ}$ centered on the GC) and inner bipolar dusty structure at $8.3 \mu \mathrm{m}$ (right; $3^{\circ} \times 3^{\circ}$ ). (e) $\mathrm{CS}(2-1)$ polar arc and hourglass structure in the central $0.8^{\circ} \times 0.6^{\circ}$. (f) Vertical $\left( \pm 0.2^{\circ}\right)$ velocity structure of the CS $(2-1)$ polar arc, averaged over Galactic longitudes $l=[-0.18,0.07]$. (g) Deficiency in H I $21-\mathrm{cm}$ emitting gas in the central $50^{\circ} \times$ $50^{\circ}$. (h) Evidence of acceleration among the high-velocity $\mathrm{H}$ I clouds in the central $20^{\circ} \times 20^{\circ}$ around the GC. Images reproduced with permission from (a) Su et al. (2010), (d) Bland-Hawthorn and Cohen (2003), (e, f) Hsieh et al. (2016), (g) Lockman and McClure-Griffiths (2016), (h) Lockman et al. (2019), copyright by AAS; (b) Ponti et al. (2019), (c) Heywood et al. (2019), copyright by the author(s).

The first evidence for a cooler component associated with this large-scale outflow was reported by Bland-Hawthorn and Cohen (2003), who showed the existence of a

6 For instance, Krumholz et al. (2017a) argue that the average SFR is closer to $\sim 1 M_{\odot} \mathrm{yr}^{-1}$, more in line with the estimated mass supply rate to the $\mathrm{CMZ}\left(\gtrsim 1 M_{\odot} \mathrm{yr}^{-1}\right.$, Sormani and Barnes 2019). 
limb-brightened bipolar structure of cool $(\sim 20-30 \mathrm{~K})$ dust extending $\pm 1^{\circ} \simeq \pm 140 \mathrm{pc}$ from the mid-plane of the Galaxy, coincident with the radio-emitting Galactic Center Lobe structure (Fig. 9d). The total mass in the shells is $\sim 5 \times 10^{6} \mathrm{M}_{\odot}$, using a Galactic dust-to-gas ratio, and the kinetic energy is $\sim 1 \times 10^{56}\left(v_{\mathrm{sh}} / 100\right)^{2} \mathrm{erg}$, assuming an uncertain shell expansion velocity of $100 \mathrm{~km} \mathrm{~s}^{-1}$. The morphology, kinematics, and physical conditions of the molecular gas in the central $250 \mathrm{pc}$ of the Milky Way (the so-called Central Molecular Zone or CMZ) have since been refined considerably using a large number of molecular gas tracers (e.g., Kruijssen et al. 2015; Tanaka et al. 2018). While most of the gas kinematics in the CMZ is dominated by rotation on closed elliptical orbits and radial inflow along the stellar bar (Sormani et al. 2015; Krumholz et al. 2017a; Armillotta et al. 2019), or open streams and spiral arms (Henshaw et al. 2016), it is clear that a number of features in this region have anomalous kinematics (Henshaw et al. 2016; Oka et al. 2019; Yusef-Zadeh et al. 2019).

One of these features is the extraplanar "polar arc" detected by Hsieh et al. (2016) in the $\mathrm{CS}(2-1)$ and $\mathrm{CS}(1-0)$ lines (Fig. 9e-f). This feature extends up to $\sim 30 \mathrm{pc}$ above the GC and shows a radial velocity gradient of $\sim 5.5 \mathrm{~km} \mathrm{~s}^{-1} \mathrm{pc}^{-1}$ (from 0 to $165 \mathrm{~km} \mathrm{~s}^{-1}$ ), suggestive of gas acceleration. This feature merges at the base of the Galactic disk with a "molecular loop" with opposite (blueshifted) velocities, and has elevated CS (2-1)/CS (1-0) ratios that are clearly distinct from that of the rotationallydominated central nuclear disk (CND). Another, fainter bubble is seen $\sim 7 \mathrm{pc}$ below the GC with apparent expansion velocity of $\sim 50 \mathrm{~km} \mathrm{~s}^{-1}$. The scale and velocities of these anomalous features point to an expanding outflow with a timescale of a few $10^{5} \mathrm{yr}$, slightly shorter than the estimate of Bland-Hawthorn and Cohen (2003). The $\mathrm{CS}(2-1)$ material at systemic velocity also shows a remarkable hourglass geometry suggestive of a biconical $( \pm 6 \mathrm{pc})$ cavity evacuated by a recent outflow. In contrast, the molecular gas on smaller scales shows surprisingly little evidence of outflowing kinematics (Goicoechea et al. 2018). Similarly, there is little to no evidence for outflowing motion in the material making up the warm ionized gas streamers traced for instance by [Ne II] $12.8 \mu \mathrm{m}$ (Lacy et al. 1991; Zhao et al. 2009; Irons et al. 2012). Clearly, the event that took place a few $10^{5}-10^{6}$ yrs ago is not active anymore.

In parallel to this effort to map the large-scale molecular outflow in the GC, a number of studies have searched for, and successfully detected, the neutral and warmionized gas phases entrained in this outflow. The inner $3 \mathrm{kpc}$ of the Milky Way disk have long been known to be deficient in diffuse HI 21-cm emitting gas (Lockman 1984), and recent work by Lockman and McClure-Griffiths (2016) has revealed an anti-correlation between $\mathrm{HI}$ and $\gamma$-ray emission suggesting a physical connection between the Fermi Bubbles and the HI central void (Fig. 9g). A population of $\sim 200$ compact high-velocity $\mathrm{HI}$ clouds has been detected within $\pm 1.5 \mathrm{kpc}$ of the Galactic plane, centered on the GC (McClure-Griffiths et al. 2013; Di Teodoro et al. 2018; Lockman et al. 2019). Di Teodoro et al. (2018) have argued that the kinematics of these clouds do not follow Galactic rotation but are instead consistent with a simple GC-centered biconical volume-filling outflow model with a constant radial velocity of $\sim 330 \mathrm{~km} \mathrm{~s}^{-1}$ and an opening angle $>140^{\circ}$. These clouds show a broad range of properties $\left(r=10-40 \mathrm{pc}, N_{\mathrm{HI}}=10^{18.2}-10^{19.7} \mathrm{~cm}^{-2}, n_{\mathrm{HI}}=0.3-25 \mathrm{~cm}^{-3}, \mathrm{M}_{\mathrm{HI}}=10\right.$ $10^{5} \mathrm{M}_{\odot}$, assuming that they are at the distance of the GC), adding up to a total mass of $\sim 10^{6} \mathrm{M}_{\odot}$ and a kinetic energy of $\sim 1.6 \times 10^{54} \mathrm{erg}$. The best-fitting model implies 
constant neutral-gas mass outflow rate of $\sim 0.1 \mathrm{M}_{\odot} \mathrm{yr}^{-1}$ and kinetic energy injection rate of $5 \times 10^{39} \mathrm{erg} \mathrm{s}^{-1}$ over the past $\sim 10 \mathrm{Myr}$. While these numbers may be off by factors of a few, given new evidence of acceleration of these HI clouds (Fig. 9h; Lockman et al. 2019), it is clear that this H I outflow is considerably less energetic than that associated with the Fermi Bubbles, and may in principle be driven by a past starburst rather than a recent AGN episode.

Using UV-bright background AGN and high Galactic-latitude stars projected behind, near, or in front of, the Fermi Bubbles, FUSE and HST have provided a complementary view of the large-scale outflow in the Milky Way over $R \sim 1-6.5 \mathrm{kpc}$ (Keeney et al. 2006; Bowen et al. 2008; Zech et al. 2008; Fang and Jiang 2014; Fox et al. 2015; Bordoloi et al. 2017a; Savage et al. 2017; Karim et al. 2018). Blueshifted and redshifted HVCs with maximum absolute LSR velocities that decrease with increasing Galactic latitude $|b|$ have been detected in absorption in both low- and highionization species in the direction of the Fermi bubbles. These data are consistent with a decelerating multi-phase outflow with initial (launch) velocity of $\sim 1000-1300 \mathrm{~km}$ $\mathrm{s}^{-1}$, where the cool and warm clouds are entrained within the flow (Bordoloi et al. 2017a). While the implied launch velocities of the UV-detected clouds exceed the velocities of the HI 21-cm clouds in the HI model of Di Teodoro et al. (2018), the dynamical time scale, or age, of the UV-detected outflow is similar ( 6-9 Myr) to that of the H I outflow, pointing to a common outflow event. Bordoloi et al. (2017a) estimate an average mass outflow rate of $\gtrsim 0.2 M_{\odot} \mathrm{yr}^{-1}$ and a total mass of cool gas entrained in both Fermi Bubbles of $\gtrsim 2 \times 10^{6} \mathrm{M}_{\odot}$, assuming $[\mathrm{Si} / \mathrm{H}] \approx[\mathrm{O} / \mathrm{H}] \approx-0.5$, no correction for depletion onto dust grains, and mirror symmetry between the bubbles (Karim et al. 2018). Using an outflow velocity of $1300 \mathrm{~km} \mathrm{~s}^{-1}$, the total kinetic energy associated with this outflow is $\sim 6 \times 10^{55} \mathrm{erg}$, and the inferred energy injection rate is $\gtrsim 2 \times 10^{41} \mathrm{erg} \mathrm{s}^{-1}$. The energy source (AGN or starburst) of this outflow is again ambiguous, e.g. a star formation episode of $\mathrm{SFR} \simeq 1 M_{\odot} \mathrm{yr}^{-1}$ sustained over $\lesssim 5$ Myr (e.g., Krumholz et al. 2017a) may be capable of driving this outflow. Moreover, these numbers remain uncertain since the small number (8) of sightlines clearly passing through the Fermi bubbles prevents a detailed 3D modeling of the outflowing gas kinematics.

Overall, most of the neutral and molecular gas entrained in the outflow will not escape the Milky Way. Instead, this material will participate in a large-scale galactic fountain (Bregman 1980; Armillotta et al. 2019), feeding the Milky Way CGM (Bland-Hawthorn and Gerhard 2016; Hodges-Kluck et al. 2016b; Zheng et al. 2019; Werk et al. 2019; Bish et al. 2019; Fox et al. 2019), before falling back onto the outer disk of the Galaxy, and providing new fuel for star formation. This may be a common occurrence in quenched galaxies, but the very subtle signatures of the cool, warm, hot, and relativistic fluids in this tenuous outflow will generally be challenging to detect in external galaxies.

\subsubsection{The Magellanic Clouds}

The Large Magellanic Cloud (LMC; $d \simeq 50 \mathrm{kpc}$ ) is a cauldron of star formation activity likely triggered by the tidal interactions with the Small Magellanic Cloud $\left(\mathrm{SMC} ; M_{\mathrm{SMC}} \simeq 1 / 10 M_{\mathrm{LMC}}\right)$ and the Milky Way $\left(M_{\mathrm{MW}} \simeq 100 M_{\mathrm{LMC}}\right)$. Fast (up to 
$\sim 200 \mathrm{~km} \mathrm{~s}^{-1}$ ) ionized outflow events have long been known to take place across the disk of the LMC, particularly around the giant H II region 30 Doradus powered by the mini-starburst centered on super star cluster R136 (e.g., Chu et al. 1993; Redman et al. 2003). The spectacular filamentary and bubbly appearance of the H I gas distribution in the LMC (e.g., Kim et al. 1999, 2003), and the connection between the H I shell velocities and the presence of $\mathrm{H}$ II regions and $\mathrm{OB}$ associations, clearly point to smallscale injection of energy from supernovae and stellar associations into the ISM of the LMC, but the question of whether these separate outflow events combine to drive a large-scale outflow has been a matter of debate over the years. For instance, StaveleySmith et al. (2003) detected a complex of HI 21-cm line emitting HVCs at $\sim-100$ $\mathrm{km} \mathrm{s}^{-1}$ from systemic velocity that project onto the positions of giant HI voids. Given the nearly face-on orientation of the disk of the LMC $\left(i=22^{\circ}\right.$; Kim et al. 1998), this is strong evidence that this material has been ejected from the disk of the LMC, but it is not clear that these HVCs are part of a large-scale outflow rather than separate events associated with individual bursts of star formation. UV studies with HST and FUSE of stars embedded in the disk of the LMC (Howk et al. 2002; Lehner and Howk 2007; Lehner et al. 2009; Pathak et al. 2011) have successfully answered this question. The high detection rate of blueshifted F/UV absorption lines found in these studies implies that the high-velocity material covers most of the galaxy, including both quiescent and active regions of star formation. These data also indicate that the high-velocity material has a multi-phase structure with neutral (O I, Fe II), weakly ionized (Fe II, N II), and highly ionized (O VI) components, and shows evidence of dust but no molecules. A recent analysis by Barger et al. (2016) of the spectrum of an AGN behind the LMC has shown that the outflow is kinematically symmetric with respect to the disk of the LMC with velocities reaching $\sim 110 \mathrm{~km} \mathrm{~s}^{-1}$ (after deprojection), perhaps large enough for some of the gas to escape the LMC (Fig. 10a; from Eq. (11), $v_{\text {esc }} \simeq 1.4-3 v_{\text {circ }} \simeq 90-200 \mathrm{~km} \mathrm{~s}^{-1}$, where $v_{\text {circ }}=65 \mathrm{~km} \mathrm{~s}^{-1}$; Kim et al. 1998) and become part of the Milky Way halo. Barger et al. (2016) estimate a total (neutral + ionized) outflowing gas mass of $\sim 1.3 \times 10^{6} \mathrm{M}_{\odot}$ and a mass outflow rate of $0.04 \mathrm{M}_{\odot} \mathrm{yr}^{-1}$, assuming a typical outflow velocity of $50 \mathrm{~km} \mathrm{~s}^{-1}$. This mass outflow rate is comparable to the star formation rate of the LMC, $\lesssim 0.2 \mathrm{M}_{\odot} \mathrm{yr}^{-1}$ (Harris and Zaritsky 2009).

The latest addition to the list of MW companions with cool outflows is the Small Magellanic Cloud (SMC; $d \simeq 60 \mathrm{kpc}$ ). McClure-Griffiths et al. (2018) recently reported the detection of a complex of $\mathrm{H} \mathrm{I} 21$-cm comet-shaped head-tail clouds, looping filaments, and compact ( $\sim 10-30 \mathrm{pc})$ high-velocity clouds, extending $\gtrsim 2 \mathrm{kpc}$ from the star-forming bar of SMC, and with measured velocities that deviate by up to 100 $\mathrm{km} \mathrm{s}^{-1}$ with respect to the portion of the galaxy nearest to these features (Fig. 10b). The total gas mass of this complex adds up to at least $\sim 1.3 \times 10^{7} \mathrm{M}_{\odot}$ or about $\sim$ $3 \%$ of the total atomic gas in this galaxy. The implied mass outflow rate, calculated without correcting for possible projection effects, is $\sim 0.2-1.0 \mathrm{M}_{\odot} \mathrm{yr}^{-1}$, up to an order of magnitude larger than the star formation rate of the SMC. Given the inclination of the SMC bar $\left(i \sim 40^{\circ}\right.$; Stanimirović et al. 2004) and the escape velocity of the SMC $\left(v_{\text {esc }} \simeq 1.4-3 v_{\text {circ }} \simeq 85-180 \mathrm{~km} \mathrm{~s}^{-1}\right.$, where $v_{\text {circ }}=60 \mathrm{~km} \mathrm{~s}^{-1}$; Stanimirović et al. 2004), McClure-Griffiths et al. (2018) have argued that nearly 20-40\% of the outflowing material may be escaping out of the SMC. This may be considered a lower limit 
(a)

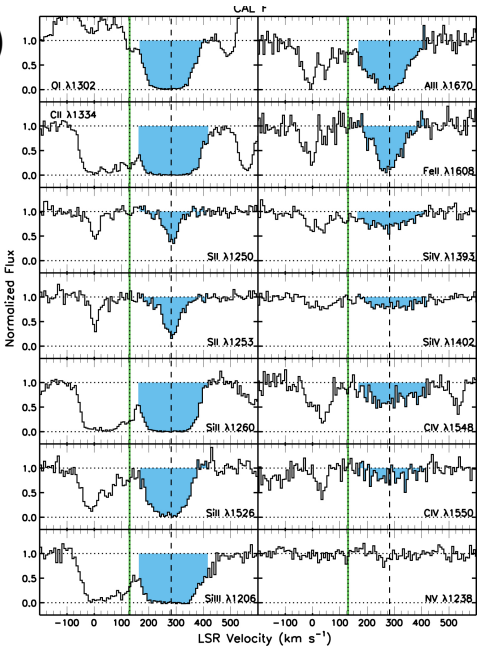

(b)

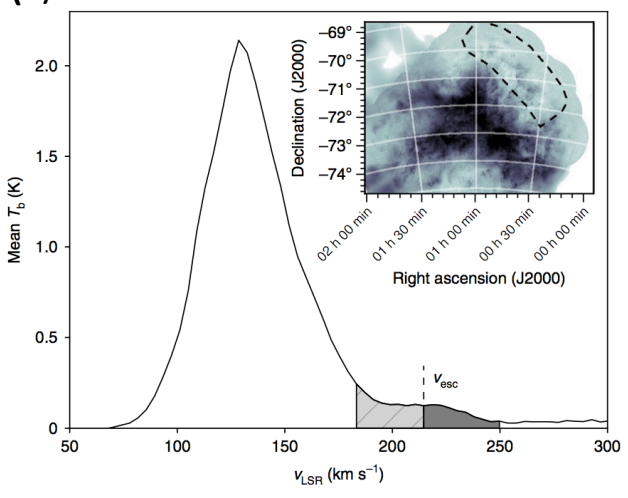

Fig. 10 Cool outflows in (a) LMC and (b) SMC. The blue shaded region in (a) marks the kinematic extent $\left(\sim \pm 100 \mathrm{~km} \mathrm{~s}^{-1}\right)$ of the multi-phase outflow in LMC, traced in both low- and high-ionization absorption lines. In (b), the inset shows the peak H I intensity image of the SMC and the dashed box indicates the region used to generate this spectrum. The dark grey shaded region in the spectrum indicates the $\mathrm{H} \mathrm{I}$ gas with velocity in excess of the escape velocity. Images reproduced with permission from (a) Barger et al. (2016), copyright by AAS; and (b) McClure-Griffiths et al. (2018), copyright by the authors.

since tidal stripping by the LMC and Milky Way, and ram-pressure forces associated with the fast $\left(\sim 300 \mathrm{~km} \mathrm{~s}^{-1}\right.$; Kallivayalil et al. 2006) motion of the SMC through the Milky Way halo, will promote the removal of any material that is outside of the host's main body. The implied dynamical timescale of the outflow ( $30-60 \mathrm{Myr})$ coincides roughly with the age of the recent burst of star formation in the portion of the bar nearest of the HI features, so it may very well have been at the origin of this outflow event. The detection of diffuse $\mathrm{H} \alpha$ and X-ray emission (Winkler et al. 2015; Sturm and Haberl 2014), as well as strong and broad O VI absorption features (Hoopes et al. 2002) throughout this region, indicates that the cool outflow is also accompanied by a warm-hot ionized gas phase. The recent detection by Di Teodoro et al. (2019) of molecular gas in two of the outflowing $\mathrm{H}$ I clouds indicates that a cold component is also associated with this outflow.

\subsection{Starburst galaxies: M 82 and NGC 253}

The nearest starburst galaxies, M 82 and NGC 253, are both hosts of multi-phase outflows. In both cases the driver of the outflow is star formation activity: neither M 82 nor NGC 253 host energetically important AGN. The proximity of both galaxies enables highly detailed views of the outflowing gas (Figs. 1 and 11). 


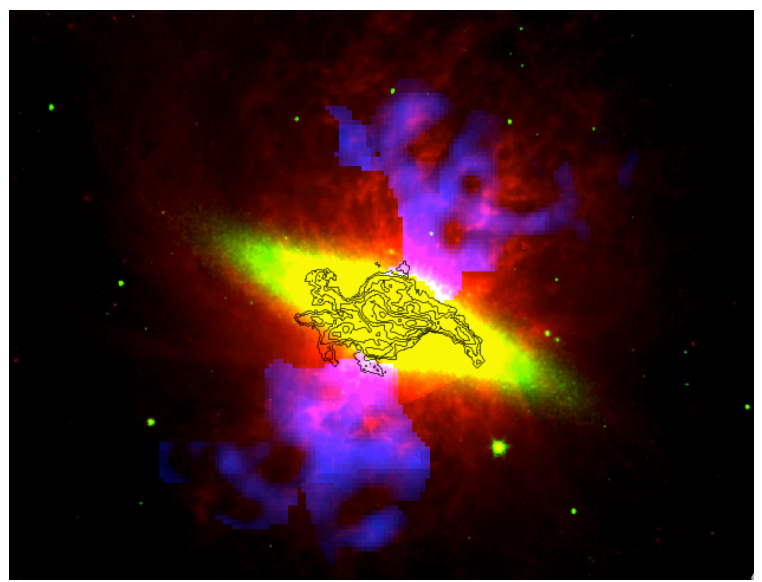

Fig. 11 Cool outflows in M 82. The red, green, and blue planes correspond to IRAC $8.0 \mu \mathrm{m}$ (PAHs), IRAC $3.6 \mu \mathrm{m}$ (stars), and H I $21 \mathrm{~cm}$ line emission masked along the minor axis to show the velocities corresponding to the outflow. Observations are from Engelbracht et al. (2006) and Leroy et al. (2015b), respectively. The contours are the extended $\mathrm{CO}$ emission from the interferometric observations by Walter et al. (2002)

\subsubsection{82}

M 82, a small galaxy part of the M 81 group, is the archetypal example of a starburst galaxy. The group is strongly interacting with $\mathrm{H}$ I gas tails connecting M 81 to the nearby M 82, NGC 3077, and NGC 2976 satellite galaxies (Yun et al. 1994; de Blok et al. 2018). The interaction has likely triggered the starburst activity in M 82. Modelling of near and mid-infrared observations indicates M 82 has a complex history (Förster Schreiber et al. 2003). It likely underwent a powerful starburst in its central $500 \mathrm{pc} 8-15 \mathrm{Myr}$ ago peaking at $160 M_{\odot} \mathrm{yr}^{-1}$, followed by a second burst in a circumnuclear ring (likely caused by a bar driving gas into the core) 4-6 Myr ago peaking at $40 \mathrm{M}_{\odot} \mathrm{yr}^{-1}$. The average recent star formation rate is $13-33 M_{\odot} \mathrm{yr}^{-1}$, depending on the contribution from low mass stars (Förster Schreiber et al. 2003).

The M 82 outflow is prominent in X-rays. Soft X-ray observations reveal an extended structure perpendicular to the major axis of the galaxy, extending several arcminutes north and south of the nucleus (e.g., Strickland et al. 1997; Strickland and Heckman 2009). Modeling of these data result in estimates for the mass loss rate of the hot wind fluid of $1.4-3.6 M_{\odot} \mathrm{yr}^{-1}$, and a terminal velocity of 1400 to 2200 $\mathrm{km} \mathrm{s}^{-1}$, much larger than the escape velocity. The inferred hot plasma temperatures are 3 to $8 \times 10^{7} \mathrm{~K}$, much hotter than a soft X-ray emitting plasma despite the cold mass loading in the central regions.

The wind is bright in warm, ionized, $\mathrm{H} \alpha$ emitting gas. This includes several filaments associated with the disk and lower wind (Shopbell and Bland-Hawthorn 1998), 
and a large nebular shell-like feature only weakly connected to the galaxy called the "H $\alpha$-cap", located at $12 \mathrm{kpc}$ over the midplane of M 82 and coincident with soft X-ray emission (Devine and Bally 1999; Lehnert et al. 1999). This feature is likely caused by a $40-80 \mathrm{~km} \mathrm{~s}^{-1}$ shock driven by the wind (Matsubayashi et al. 2012). In any case, the wind appears to move fast enough to escape, and it suggests the starburst has been active for $\gtrsim 10 \mathrm{Myr}$ and possibly much longer (Strickland and Heckman 2009).

The M 82 outflow is also prominent in far-infrared indicators of cold, dusty phases. The wind has been imaged in polycyclic aromatic hydrocarbon emission out to $6 \mathrm{kpc}$ away from the midplane with varying ratios between PAH features, suggesting some processing of the PAH carriers (perhaps grain shattering) takes place in the wind (PAH; Engelbracht et al. 2006; Yamagishi et al. 2012; Beirão et al. 2015). Modeling of the dust properties across the near-UV, optical, and infrared finds that the UV/optical emission in the wind cone is caused by scattering from dust grains entrained in the flow (Hutton et al. 2014), and it also points to grain processing. The dependence of the scattering on wavelength is best fit by either a steep power-law of grain size, or by a model with a flatter power-law and a maximum size for the grains, suggesting that large grains are missing from the entrained material. Polarization in the far-infrared dust emission in both the disk and the outflow is observed to be a few percent at $53 \mu \mathrm{m}$ to a few tenths of a percent at $154 \mu \mathrm{m}$ (where the central region is much more depolarized, Jones et al. 2019b). The polarization traces an ordered component of the magnetic field that is mostly oriented along the axis of the outflow, perpendicular to the minor axis of M 82. Spectropolarimetric measurements in the optical emission lines suggest that the dust is moving substantially slower than the ionized gas in the wind of M 82 (Yoshida et al. 2011, 2019).

Near infrared $\mathrm{H}_{2}$ ro-vibrational transitions with $E / k$ of a few thousand degrees Kelvin have been observed in the outflow, likely collisionally excited by a combination of UV-heating from the central starburst and shocks (Veilleux et al. 2009b). Lower energy mid-infrared $\mathrm{H}_{2}$ rotational quadrupole transitions are also observed, with ratios that suggest they are excited by relatively slow velocity shocks with $v_{\text {shock }} \sim 40 \mathrm{~km} \mathrm{~s}^{-1}$ (Beirão et al. 2015). The entrained warm molecular gas mass observed in the outflow is estimated to be 0.5 to $1.7 \times 10^{7} \mathrm{M}_{\odot}$.

The colder phases of the M 82 wind are also bright in $\mathrm{CO}$ and $\mathrm{H} \mathrm{I}$ emission. Walter et al. (2002) imaged the inner regions of the starburst and outflow, revealing a complex geometry of molecular streamers including faint emission along the outflow. Modeling of the $\mathrm{CO}$ excitation suggests densities $n \sim 10^{3} \mathrm{~cm}^{-3}$ and warm temperatures of up to $100 \mathrm{~K}$ (Weiß et al. 2005b). The complexity of the cold neutral wind of $\mathrm{M} 82$ is also reflected in the multi-component $\mathrm{Na} \mathrm{D}$ absorption line profiles (Schwartz and Martin 2004). Leroy et al. (2015b) find CO emission out to $3 \mathrm{kpc}$ away from the starburst, and significant $\mathrm{HI}$ emission associated with the outflow out to $5 \mathrm{kpc}$ in the north and $10 \mathrm{kpc}$ in the south. The kinematics show line splitting, likely indicative of a tilted bi-conical geometry. The speed of the molecular outflow is critically dependent on the inclination assumed, but likely $v \sim 300-450 \mathrm{~km} \mathrm{~s}^{-1}$. Leroy et al. (2015b) find a decreasing mass loss rate in the outflow as a function of distance to the mid-plane, rapidly decreasing for $\mathrm{H}_{2}$ traced by $\mathrm{CO}$, and more slowly decreasing for $\mathrm{HI}$ and for total gas as inferred from the dust continuum. The total mass outflow rate in the colder phases seems to be considerably higher than that in the hot wind fluid, 
as modeled by Strickland and Heckman (2009). Martini et al. (2018) point out that the H I kinematics are not compatible with an outflow launched only from the nuclear region, but instead require launching over a $\sim 1 \mathrm{kpc}$ region that matches better the extent of the starburst. The kinematics are also not compatible with $\mathrm{H}$ I formation through cooling from the much faster X-ray emitting hot phase. Moreover, Martini et al. find that the $\mathrm{H}$ I is decelerating along the outflow: this is particularly well constrained on the more extended south side emission. The deceleration experienced by the $\mathrm{H} \mathrm{I}$ is too large to be due to gravity alone, suggesting that the ejected material is experiencing other drag forces, perhaps from pre-existing material associated with tidal debris. This casts further doubt on what fraction, if any, of the cold material may escape the system (Martini et al. 2018).

\subsubsection{NGC 253}

NGC 253, the Sculptor galaxy, is another prototypical example of a nearby starburst $(d \sim 3.5 \mathrm{Mpc}$; Rekola et al. 2005). It is a barred galaxy and its starburst is circumnuclear, meaning it is highly concentrated in its central 200-300 pc. The star formation rate in this region is $\sim 2-3 M_{\odot} \mathrm{yr}^{-1}$ (Ott et al. 2005; Leroy et al. 2015a; Bendo et al. 2015), and there is no evidence for an energetically important AGN. High resolution submillimeter-wave imaging reveals a complex of massive clusters in formation in the central $200 \mathrm{pc}$ (Leroy et al. 2018), which encompass a large fraction of the total nuclear star formation activity. A bi-conical wind emerges out of this region, apparent in X-rays (Strickland et al. 2000, 2002), $\mathrm{H} \alpha$ emission and other ionized lines (Sharp and Bland-Hawthorn 2010; Westmoquette et al. 2011), neutral sodium emission (Heckman et al. 2000), polycyclic aromatic hydrocarbon emission (Tacconi-Garman et al. 2005), OH absorption (Turner 1985; Sturm et al. 2011), and spatially resolved molecular emission (Fig. 1e; Bolatto et al. 2013a; Walter et al. 2017; Zschaechner et al. 2018; Krieger et al. 2019). The geometry of the wind is well characterized through $\mathrm{H} \alpha$ observations, emerging approximately perpendicular to the plane of the galaxy and almost in the plane of the sky (inclination of $12^{\circ}$ ) with an opening angle of $\sim 60^{\circ}$. NGC 253 has prominent lobes associated with its outflow, extending to distances of $\sim 10 \mathrm{kpc}$ away from the central region along the galaxy minor axis. The extended emission is observed in X-rays, far-UV, $\mathrm{H} \alpha$, and radio-synchrotron (Bauer et al. 2007; Kapińska et al. 2017).

The molecular outflow in NGC 253 has a CO-emitting mass of $\sim 3-4 \times 10^{7} \mathrm{M}_{\odot}$, and molecular mass loss rates estimated to be $\sim 20 \mathrm{M}_{\odot} \mathrm{yr}^{-1}$ (Zschaechner et al. 2018; Krieger et al. 2019). By comparison, the mass of the $\mathrm{H} \alpha$-emitting phase is $\lesssim 10^{7} \mathrm{M}_{\odot}$ (Westmoquette et al. 2011), and the inferred mass loss rate is $\sim 4 \mathrm{M}_{\odot} \mathrm{yr}^{-1}$ (Krieger et al. 2019). The mass-loss rate in the hot, X-ray emitting fluid is estimated to be < $2.2 \mathrm{M}_{\odot} \mathrm{yr}^{-1}$ for reasonable flow parameters (Strickland et al. 2000). As a consequence the cold, slower phase carries appears to dominate the mass loss from the central starburst region.

Both the $\mathrm{H} \alpha$ and the CO-emitting material appear to be located in the walls of the hot, X-ray emitting outflow, at least in the inner $0.5 \mathrm{kpc}$ of the flow. Westmoquette et al. (2011) report a physical outflow speed for the $\mathrm{H} \alpha$ emitting material of $\sim 200-300 \mathrm{~km} \mathrm{~s}^{-1}$. They measure a velocity gradient that they interpret as accelera- 
tion, with a magnitude of $\sim 0.6 \mathrm{~km} \mathrm{~s}^{-1}$ per parsec along the axis of the outflow cone. Walter et al. (2017) measure a very similar (within de-projection uncertainties) velocity gradient of $\sim 1 \mathrm{~km} \mathrm{~s}^{-1}$ per parsec for the molecular material along their brightest filament. It remains unclear what fraction of this material reaches escape speed, if any. The existence of the extended lobes suggests that at least part of the flow can reach very far distances from the starburst regions.

Walter et al. (2017) identify several molecules in the molecular outflow, including species usually associated with dense gas such as $\mathrm{HCN}$. They also point out that the line ratios, to the extent they can measure, are similar in the filamentary ejecta and in the central starburst. This suggests the molecular material may be entrained from the central regions, rather than being formed in situ through condensation which would require very fast chemistry to recreate the implied molecular abundances. Walter et al. (2017) also calculate the expected acceleration due to radiation pressure likely experienced by the material in the brightest filament. Their conclusion is that radiation pressure cannot be the main source of momentum to explain the observed ejection velocity, as it would require an extremely favorable and unlikely geometry.

\subsection{Seyfert Galaxy: NGC 1068}

NGC 1068 is a nearby $\left(d=14 \mathrm{Mpc}, 1^{\prime \prime}=70 \mathrm{pc}\right)$ galaxy with a nuclear bar and a massive pseudo-bulge (Kormendy and Ho 2013) that is often regarded as the prototypical Seyfert 2 galaxy. It has a prominent starburst ring of $\sim 1-1.5 \mathrm{kpc}$ radius and a $200 \mathrm{pc}$ molecular circumnuclear disk surrounding the AGN (e.g., Schinnerer et al. 2000). Non-circular motions as traced by $\mathrm{CO}$ and also near-IR $\mathrm{H}_{2}$ in the inner regions can be interpreted as due to in- and outflow motions (Galliano and Alloin 2002; Davies 2008; García-Burillo et al. 2010; Krips et al. 2011). The nuclear gas is dense $\left(n>10^{5-6} \mathrm{~cm}^{-3}\right)$ and can be probed by polar molecules such as HCN and high- $J$ CO emission. Studies suggest that the chemistry and physical conditions of the gas is strongly impacted by the AGN, either by X-rays or shocks (e.g Tacconi et al. 1994; Sternberg et al. 1994; Usero et al. 2004; Aalto et al. 2011; Viti et al. 2014; García-Burillo et al. 2017). The nucleus is launching a radio jet that is (on small scales) perpendicular to the nuclear water maser disk (Gallimore et al. 1997) and creates a bow shock on 100 pc scales.

While the AGN in NGC 1068 is not as powerful as quasars (which are thought to drive outflows energetic enough to quench star formation in their massive hosts), it is much closer than any quasar (e.g. the closest quasar, Mrk 231 discussed in the next section, is more than ten times more distant) and therefore it can be investigated in far greater detail. Moreover, it offers the possibility of investigating both the outflow associated with the radio jet and the radiatively driven component of the outflow.

The presence of an ionized outflow has been clearly detected by various authors, through optical, UV and near-IR nebular tracers, in the shape of a biconical or hourglass structure co-aligned with the radio-jet (NE-SW), with velocities of several hundred $\mathrm{km} \mathrm{s}^{-1}$, exceeding 1,000 $\mathrm{km} \mathrm{s}^{-1}$ in some locations (e.g. Pogge 1988; Cecil et al. 1990; Crenshaw and Kraemer 2000; Tecza et al. 2001; Cecil et al. 2002; Das et al. 2006; Zheng et al. 2008; Barbosa et al. 2014; Vermot et al. 2019). However, one pecu- 
liarity is that the high ionization lines and lower ionization lines, tracing denser gas, have opposite velocities. For instance in the NE cone (which is the one on the near side of the disk), [O III] $5007 \AA$ is blueshifted while [Fe II] $1.64 \mu \mathrm{m}$ is redshifted. This has been interpreted (Fig. 12d) as evidence that the bow shock generated by the radio jet accelerates low-density, more highly ionized gas (being directly exposed to the AGN ionizing radition) out of the galactic plane in our direction, while the bow shock on the far side ploughs into the galactic disk and accelerates denser and less-ionized gas in the opposite direction.

Recent high-resolution ALMA observations of NGC 1068 reveal molecular outflows on what may be several scales. García-Burillo et al. (2014) mapped various high transitions of $\mathrm{CO}, \mathrm{HCN}, \mathrm{HCO}^{+}$and $\mathrm{CS}$ showing motions of dense gas, on scales from 50 to $400 \mathrm{pc}$, that deviate from rotation (Fig. 12a). This emission is consistent with the outflow previously traced by the low ionization tracers, i.e. in the opposite direction of the high ionization transitions (e.g. [O III] $5007 \AA$ ). One interpretation is that this apparently outflowing molecular gas is also part of the dense gas in the galactic disc that is being accelerated by the oblique bow shock (Fig. 12d). This molecular outflow is estimated to have $\dot{M} \sim 63 M_{\odot} \mathrm{yr}^{-1}$, which is an order of magnitude higher than the star formation rate at these radii. Nuclear depletion times are short (less than $1 \mathrm{Myr}$ ). The inner gas reservoir may be rapidly replenished by inflows from the outer disk, especially given the streaming gas resulting from the torques exerted by the stellar bar. A fast $\left(\sim 400 \mathrm{~km} \mathrm{~s}^{-1}\right)$ molecular outflow has been detected on smaller scales, a few parsecs from the nucleus (Fig. 12b; Gallimore et al. 2016), but this nuclear molecular outflow has velocities in the opposite direction as that of the molecular outflow on large scales, i.e. going out of the disk. This nuclear molecular outflow appears to be disconnected from the molecular outflow on larger, 100-pc scales, and may have a different origin. While the large-scale molecular outflow is associated with gas in the galactic disk accelerated by the bow shock entering into the disc, the nuclear molecular outflow is likely associated with nuclear clouds being directly accelerated by the AGN radiation pressure or ram pressure from the nuclear disk-wind (as in the disk-wind scenario proposed by Elitzur and Shlosman 2006).

Garcia-Burillo et al. (2019) have recently presented a model of the nuclear wind (the outflowing torus) as well as an update of the 2014 jet expansion model (Fig. 12d). A vertical component, in addition to the co-planar flow, is now included, that can explain the $\mathrm{CO}$ line-splitting and large line widths. The model can also account for the velocity structure of the highly ionized wind. This wind encounters less resistance against expansion above and below the disk mid-plane resulting in opposite velocities with respect to CO. Garcia-Burillo et al. also find that half of the mass of the torus is outflowing (Fig. 12e), which means that this AGN feedback on very small scales is also regulating the fueling and thus likely setting the AGN flickering timescale.

Interestingly, the pc-scale nuclear wind has also been directly detected as a PCygni profile of the $\mathrm{HCN}(3-2)$ transition against the nuclear radio core (Fig 12c), therefore implying that the nuclear outflowing gas must be very dense $\left(\sim 10^{7} \mathrm{~cm}^{-3}\right.$; Impellizzeri et al. 2019). The fact that such dense molecular clouds are already outflowing at high velocities $\left(\sim 450 \mathrm{~km} \mathrm{~s}^{-1}\right)$ so close to the nucleus, where the dynamical timescales (a few thousand years) are far too short for the formation of molecules, favor the scenario of outflowing molecular clouds resulting from acceleration of cir- 
(a)

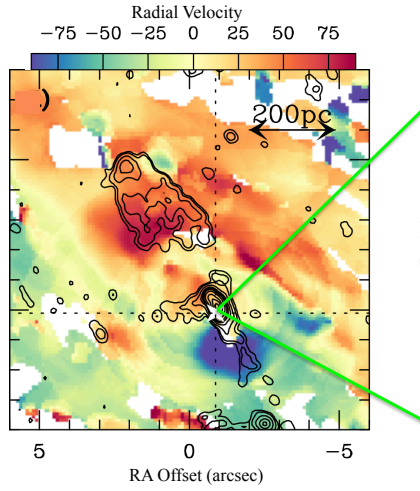

(b)

(c)

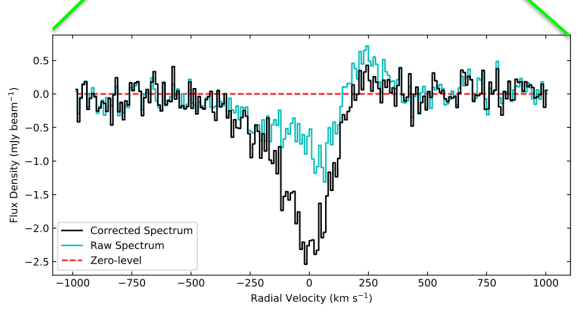

(d)

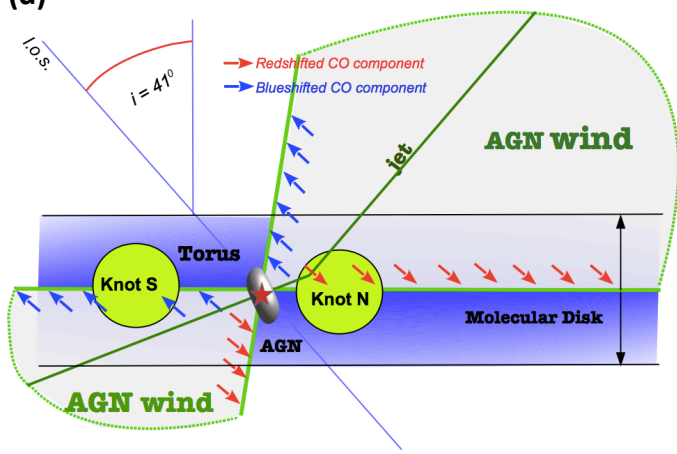

(e)

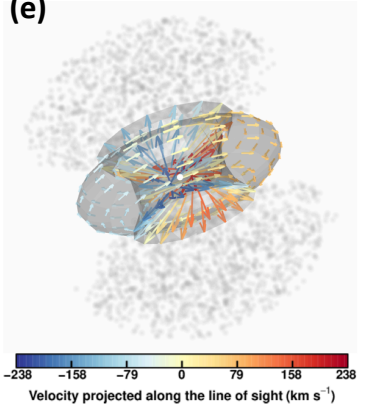

Fig. 12 Cold outflows in NGC 1068. (a) Residual velocity field (after subtracting the rotation field) traced by the $\mathrm{CO}(3-2)$ line, with the $22 \mathrm{GHz}$ radio map overlayed with contours. Adapted from García-Burillo et al. (2014). (b) Location of different kinematics components in the central few pc of NGC 1068; blue $v$ $<-70 \mathrm{~km} \mathrm{~s}^{-1}$, red $v>70 \mathrm{~km}^{-1}$, yellow $-70 \mathrm{~km} \mathrm{~s}^{-1} \leq v \leq 70 \mathrm{~km} \mathrm{~s}^{-1}$; circles for $\mathrm{CO}(6-5)$, diamonds for the nuclear water maser disk. Note that the $\mathrm{CO}(6-5)$ outflow has inverted velocity profile with respect to the large-scale molecular outflow. Adapted from Gallimore et al. (2016). (c) HCN(3-2) profile extracted from the central $1.2 \mathrm{pc}$, at the location of the radio core, showing a clear P-Cygni profile. Adapted from Impellizzeri et al. (2019). (d) Sketch of the central region of NGC 1068 illustrating the outflow on large $(100 \mathrm{pc})$ scales, driven by the expansion of the bow-shock generated by the radio-jet. (from Garcia-Burillo et al. 2019). (e) Sketch of the kinematic model of the molecular torus described in Garcia-Burillo et al. (2019). Images reproduced with permission from (a) García-Burillo et al. (2014) and (d, e) Garcia-Burillo et al. (2019), copyright by ESO; and (b) Gallimore et al. (2016) and (c) Impellizzeri et al. (2019), copyright by AAS. 
cumnuclear clouds rather than formation of molecular clouds from gas cooling inside the hot outflow, at least on such small scales. Clearly, in NGC 1068 jet/bow-shock driven molecular outflow and radiation-driven molecular outflow are both present at the same time and both of them are relevant for the evolution of the nuclear region.

\subsection{Quasar: Mrk 231}

Mrk 231 is the nearest quasar known $\left(z=0.0422 ; d \simeq 180 \mathrm{Mpc} ; 1^{\prime \prime}=0.867 \mathrm{kpc}\right)$ and arguably the best example of a "wind-dominated" fast-accreting $\left(L / L_{\text {Edd }} \simeq 1\right)$ quasar where the signatures of the AGN-driven outflow are detected on virtually all spatial scales, from the AGN accretion disk to the CGM. As such, Mrk 231 offers a unique laboratory to study a phenomenon that might be more common in the early universe during the epoch of formation and fast growth of SMBHs. Mrk 231 is the end-product of a (locally) rare infrared luminous gas-rich galaxy merger which provided the right conditions to trigger both a luminous AGN and a powerful circumnuclear starburst $\left(\dot{M}_{*} \sim 140 \mathrm{M}_{\odot} \mathrm{yr}^{-1}\right.$; this starburst accounts for about $30 \%$ of the bolometric luminosity of the system; e.g., Veilleux et al. 2009a).

As shown in Figure 13a, broad spatially unresolved blueshifted absorption line features are detected in the nucleus across the range $\sim-3500$ to $-6000 \mathrm{~km} \mathrm{~s}^{-1}$ in several transitions including many low-ionization species (e.g. Na I D 5890, 5896, He I* 3889 and 10830, Ca II H and K, Mg II 2796, 2803, and Mg I 2853, as well as several NUV Fe II features, hence fitting the rare FeLoBAL category; e.g., Boksenberg et al. 1977; Smith et al. 1995; Gallagher et al. 2002, 2005; Rupke et al. 2002; Veilleux et al. 2013b, 2016; Leighly et al. 2014). Dust within the outflowing clouds is required to provide shielding for Na I D and also explain the blueshifted Ly $\alpha$ and C IV line emission. The presence of excited-state Fe II UV60, 61, 62, 63, and 78 and He I* 3889 absorption lines leads to a distance of $\lesssim 2-20 \mathrm{pc}$ between the absorber and the ionizing source and implies a mass outflow rate and kinematic luminosity of the BAL outflow of $\lesssim 10-100 \mathrm{M}_{\odot} \mathrm{yr}^{-1}$ and $\lesssim 10^{44-45} \mathrm{erg} \mathrm{s}^{-1}$ (Veilleux et al. 2016). Other indicators of outflows in the nucleus include Blazar-like radio flares at $20 \mathrm{GHz}$, associated with highly relativistic ejecta on pc scale (Reynolds et al. 2009, 2013), and the possible (3.5- $\sigma$ ) detection of a P-Cygni Fe $\mathrm{K} \alpha 6.7 \mathrm{keV}$ profile, indicative of an ultra-fast ( $20,000 \mathrm{~km} \mathrm{~s}^{-1}$ ) outflow on smaller scales (0.001-0.01 pc; Feruglio et al. 2015).

A slower $\left(\lesssim 1500 \mathrm{~km} \mathrm{~s}^{-1}\right)$, galactic-scale $(\sim 0.1-3 \mathrm{kpc})$ cool-gas outflow has been detected in Mrk 231 using multiple techniques (Fig. 13b-g): neutral-gas absorption line tracers (Na I D: Rupke et al. 2002; Rupke and Veilleux 2011; Rupke et al. 2017; Morganti et al. 2016, in HI), molecular-gas FIR absorption line tracers (Fischer et al. 2010; Sturm et al. 2011; González-Alfonso et al. 2014, 2017b), and molecular-gas mm-wave emission line tracers (Feruglio et al. 2010, 2015; Aalto et al. 2012, 2015a; Cicone et al. 2012; Alatalo 2015; Lindberg et al. 2016). The inferred cool-gas mass outflow rate ranges from $\sim 100-200 \mathrm{M}_{\odot} \mathrm{yr}^{-1}$ (Na I D tracer; Rupke et al. 2017) to 500-1000 $\mathrm{M}_{\odot} \mathrm{yr}^{-1}$ (OH and CO tracers; González-Alfonso et al. 2017b; Feruglio et al. 2015), and the corresponding kinetic energy rate of the cool outflow is $\sim 0.2 \%$ (Na I) and $\sim 1-3 \%$ of the AGN bolometric luminosity. Remarkably, the warm and hot ionized components of the large-scale outflow are energetically insignificant in 
(a)

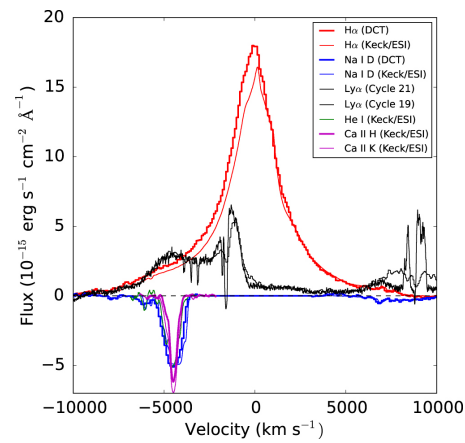

(c)

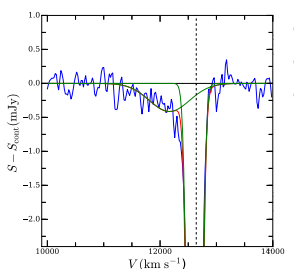

(d) (b)

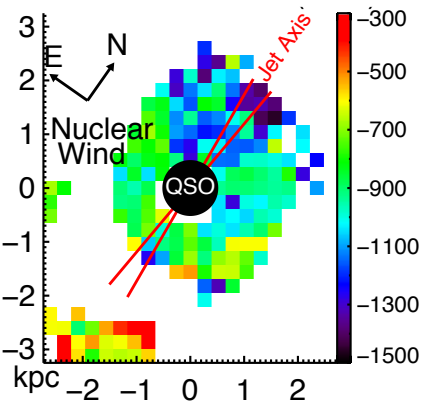

(e)

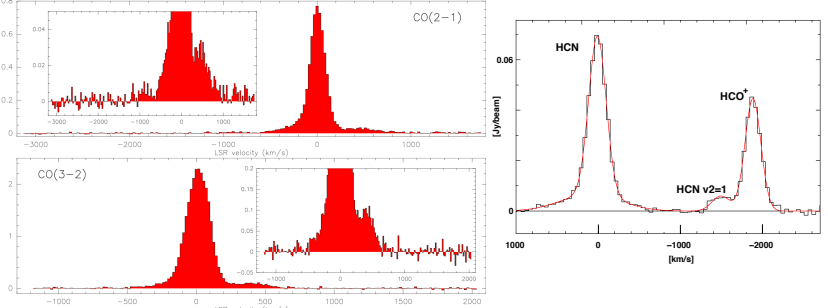

(f)

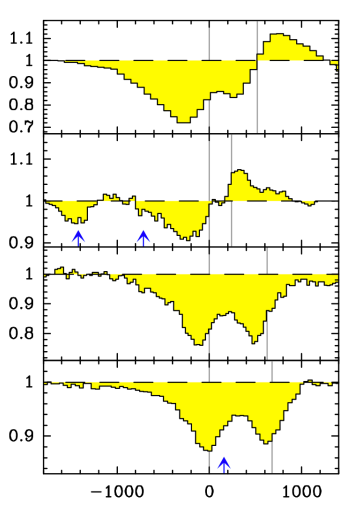

(g)

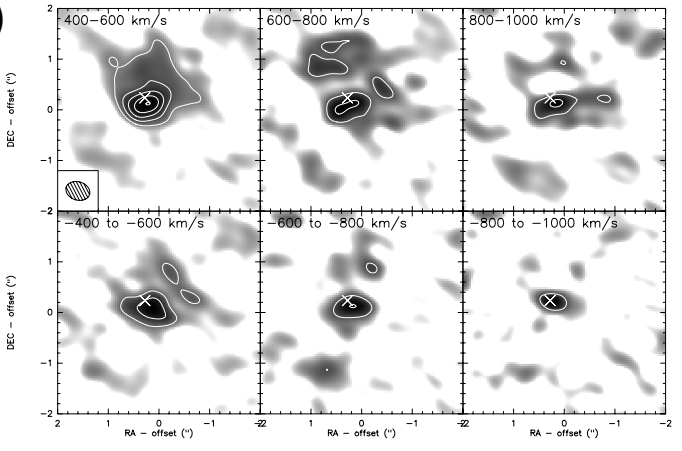

Fig. 13 The nuclear and large-scale cool outflows of Mrk 231. (a) Broad low-ionization absorption features in the central AGN. (b) Spatially resolved velocity field of the Na I D absorption line. (c) Blue wing in H I 21-cm absorption line. (d) Blue and red wings in the $\mathrm{CO}$ (1-0) and (2-1) line profiles. (e) HCN signatures of the outflow. (f) $\mathrm{OH}$ signatures of the outflow. (g) Spatially resolved blue- and red-shifted $\mathrm{CO}$ line emission. Images reproduced with permission from (a) Veilleux et al. (2016), (b) Rupke and Veilleux (2011), (f) González-Alfonso et al. (2017b), copyright by AAS; and (c) Morganti et al. (2016), (d, g) Feruglio et al. (2015), (e) Aalto et al. (2015a), copyright by ESO.

comparison to the cool-gas component, barely detected in $\mathrm{H} \alpha$ (e.g., Rupke et al. 2017) and the X-rays (Veilleux et al. 2014), respectively. Overall, the energetics of the largescale outflow strongly suggest that the AGN in Mrk 231 plays a dominant role in driving this powerful outflow event.

The velocities, masses, and energetics of the neutral and molecular components of the large-scale outflow in this object significantly exceed those discussed thus far 
in this section. This is not surprising given the extremely powerful source of energy at the center, but this is also due to the remarkably dense and compact mergerinduced dust-obscured environment encompassing this energy source $\left(N\left(\mathrm{H}_{2}\right) \sim 1 \times\right.$ $10^{24} \mathrm{~cm}^{-2}$ in the inner $\sim 100 \mathrm{pc}$; Aalto et al. 2015a), which helps capture an unusually large fraction of the radiative and mechanical energy produced by the quasar. These special conditions may also help explain the other distinctive characteristics of this outflow, including the virtual lack of an ionized component and the fact that the cool outflowing gas clouds are embedded in the flow itself rather than distributed along edge-brightened biconical structures like those in M82 and NGC 253. The cooler molecular portion of the outflow in Mrk 231 is also denser on average (up to $\sim 5 \times 10^{5} \mathrm{~cm}^{-3}$ ) than those in these two objects, and shows velocity-dependent $\mathrm{HCN} / \mathrm{HNC} / \mathrm{HCO}^{+}$ratios, signs of chemical differentiation possibly due to shocks (Sect. 3.4.5; Lindberg et al. 2016). Comparisons of the mm-wave spectra with the predictions of RADEX non-LTE radiative transport models (van der Tak et al. 2007) suggests that the molecular clouds making up this portion of the outflow can be represented as self-gravitating dense clouds, if a normal, Galactic $\mathrm{HCN}$-to- $\mathrm{H}_{2}$ abundance is assumed, but this result can be relaxed if $\mathrm{HCN}$ abundances are enhanced by shocks or X-rays from the central quasar (Sect. 3.4.5; Aalto et al. 2015a). A recent study reveal very bright $\mathrm{CN}$ emission from the Mrk 231 outflow. This may be due to UV photons originating from star formation in the outflow itself, or from the quasar (Cicone et al. 2019). González-Alfonso et al. (2018) have recently argued that the high $\mathrm{OH}^{+}$abundances relative to $\mathrm{OH}, \mathrm{H}_{2} \mathrm{O}$, and $\mathrm{H}_{3} \mathrm{O}^{+}$measured from the Herschel spectra imply a high ionization rate that cannot be explained by the unattenuatd hard $\mathrm{X}$-ray flux measured in this object (Teng et al. 2014). They suggest instead that lowenergy (10-400 Mev) cosmic-rays accelerated in the forward shock associated with the molecular outflow are responsible for the ionization.

The current best estimate for the momentum injection rate of the large-scale outflow in Mrk 231 exceeds by a factor of 5-10 the total radiation pressure $L / c$ from the central energy source (quasar + starburst; Feruglio et al. 2015; González-Alfonso et al. 2017b; Fluetsch et al. 2019), and thus favors the energy-driven scenario discussed in Sect. 2.2.1. A comparison of the kinetic energy of the X-ray nuclear wind with that of the cool gas outflow (using Eq. (8) from Sect. 2.2.1) implies that most of the kinetic energy in the X-ray wind goes into bulk motion of the swept-up molecular material (Feruglio et al. 2015), although it is important to repeat that the detection of the X-ray wind in this system is only at the $3.5-\sigma$ level.

\subsection{Jetted AGN: Centaurus A, IC 5063, NGC 1266, and NGC 1377}

\subsubsection{Centaurus A}

At a distance of only $\sim 3.8 \mathrm{Mpc}$ (Harris et al. 2010, $1^{\prime \prime}=19 \mathrm{pc}$, similar to that of M82 and NGC 253), Centaurus A or Cen A for short is the nearest radio galaxy and a classic example of jetted energy making its way through the ISM of the host galaxy, which in this case is an elliptical galaxy with a prominent dust lane and a complex of HI-rich tidal debris left over from the merger or stripping interaction 
of a small gas-rich galaxy 250-750 Myr ago (e.g., Schiminovich et al. 1994; Struve et al. 2010; Morganti and Oosterloo 2018). Cen A is a low-power Fanaroff type I radio source so it is perhaps not surprising to find very little evidence of outflowing cool gas in the central inner kpc of this object. The dynamics of both the neutral material (traced by H I $21 \mathrm{~cm}$ line emission and absorption) and molecular gas (traced by $\mathrm{CO}, \mathrm{HCO}^{+}, \mathrm{HCN}$, and $\mathrm{HNC}$ absorption features) are dominated by a rotating circumnuclear disk and intervening material likely associated with the dust lane (e.g., Liszt 2001; Morganti et al. 2008; Espada et al. 2010). The only cool-gas kinematic features that may not fit this picture are the moderately broad (FWHM $\sim 50 \mathrm{~km} \mathrm{~s}^{-1}$ ) $\mathrm{H} \mathrm{I}$ and $\mathrm{HCO}^{+}$absorption lines associated with the base of the nuclear jet, located $10 \mathrm{pc}$ from the AGN, where molecular hydrogen appears to be efficiently dissociated by X-rays from the AGN (Espada et al. 2010). A shell-like, bipolar structure $500 \mathrm{pc}$ to the north and south of the nucleus has also been detected by Quillen et al. (2006) in Spitzer dust maps at 8.0 and $24 \mu \mathrm{m}$, suggestive of a coherent expanding structure similar to that seen in the MW (Sect. 4.1.1), but this dusty outflow has not yet been confirmed kinematically.

The influence of the radio jet on the ISM is more apparent on larger scales. Deep UV, infrared, and optical images have revealed two linear dusty line-emitting filaments located $\sim 8$ and $\sim 15 \mathrm{kpc}$ north of the central AGN, coincident with young stars with ages $\lesssim 10 \mathrm{Myr}$ (e.g., Mould et al. 2000; Crockett et al. 2012) and well aligned with the jet radio structures on the same scale (Fig. 14a; Neff et al. 2015; Hamer et al. 2015; Salomé et al. 2016; McKinley et al. 2018). FIR emission has also been detected in a southern filament along the direction of the southern jet (Salomé et al. 2016). The disturbed kinematics of the neutral and ionized gas in the northern filaments suggest an on-going interaction with the radio jet (Oosterloo and Morganti 2005; Santoro et al. 2015b,a, 2016). These filaments have been interpreted as evidence for jet-induced star formation at the level of a few $10^{-3} \mathrm{M}_{\odot} \mathrm{yr}^{-1}$ (e.g., De Young 1981), although this is probably not the whole story. The high level of ionization in the optical filaments strongly argues for additional ionizing radiation originating from the central AGN (e.g., Sharp and Bland-Hawthorn 2010; Santoro et al. 2015b,a; McKinley et al. 2018) or shocks induced by the interaction with the radio jet (Santoro et al. 2015a; Salomé et al. 2019) or with a wide-angle starburst/AGNdriven wind that coexists with, and extends on similar scales as, the jetted outflow (Neff et al. 2015; McKinley et al. 2018).

Taken at face value, the star formation rate in the northern filaments of Cen A is 2-3 orders of magnitude less than the inferred star formation rate in the central galaxy (Salomé et al. 2016), and thus the current level of jet-induced star formation will not have a significant impact on the overall evolution of the host galaxy. Interestingly, the star formation efficiency (or $1 / t_{\text {depl }}=\dot{M}_{*} / M_{\text {mol }}$ ) in the filaments, measured from the ratio of the star formation rate to the molecular gas mass derived using a $\mathrm{CO}-$ to- $\mathrm{H}_{2}$ that is appropriate for the low metallicities of the filaments $\left(Z=0.4-0.8 Z_{\odot}\right)$, is also lower by about two orders of magnitude relative to that in the host galaxy. So it shows that, while the jet-gas interaction may be needed to compress and cool the gas to form stars, the local turbulent energy associated by this interaction (as evidenced by the broad CO line widths; Salomé et al. 2016) prevents the gas from forming new stars efficiently. 
(a)
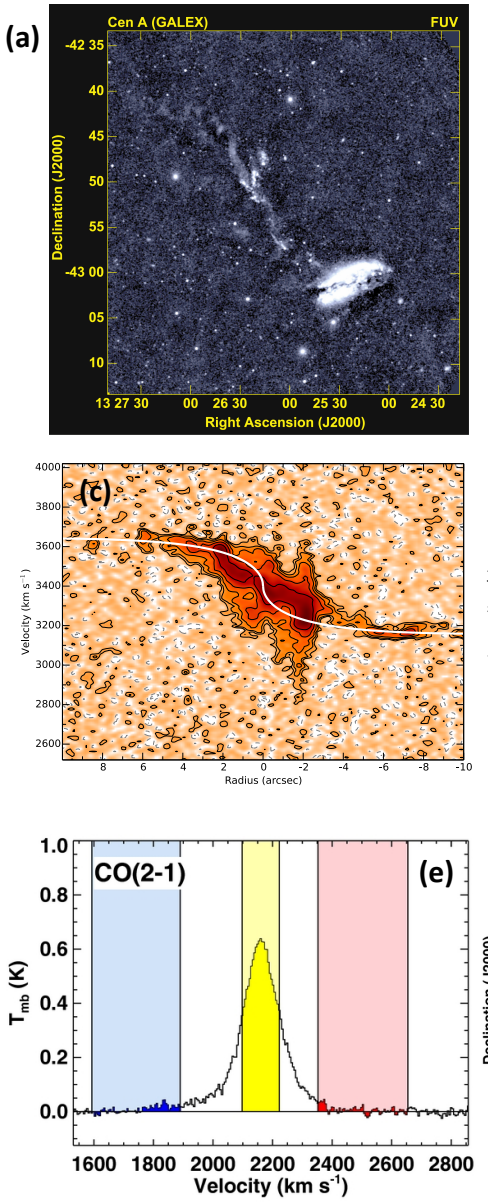

(b)
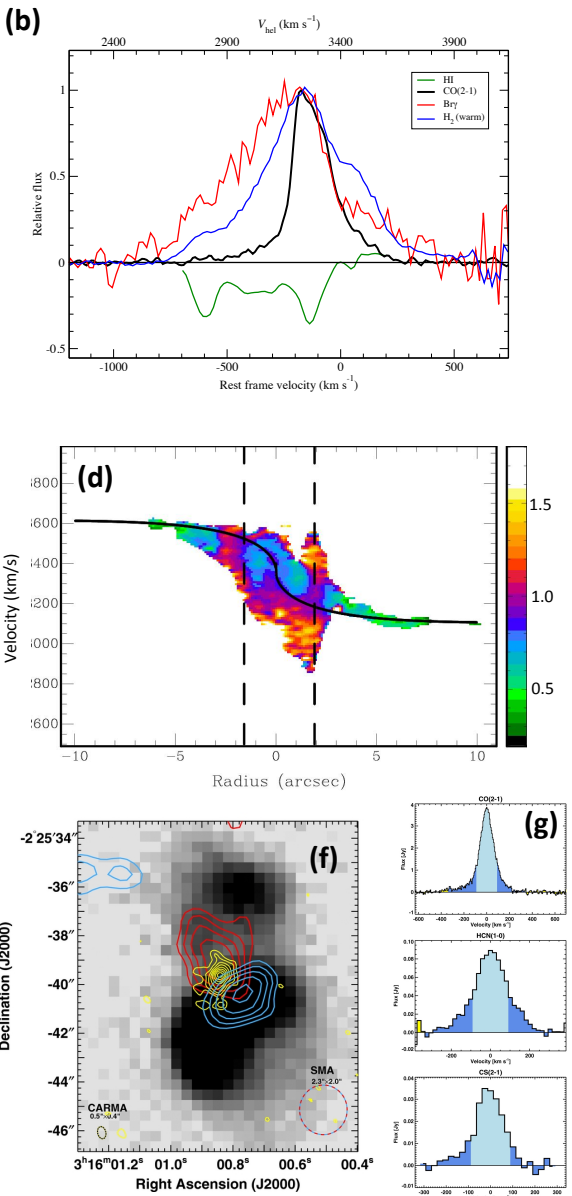

Fig. 14 Jetted cool outflows. (a) Evidence for jet-induced star formation in filaments along the radio jets of Centaurus A. (b) Multi-phase outflow of IC 5063 detected in Br $\gamma, \mathrm{H} \mathrm{I} 21 \mathrm{~cm}, \mathrm{H}_{2} 2.12 \mu \mathrm{m}$, and CO (2-1), at the location of the north-west radio lobe. (c) Position-velocity diagram of $\mathrm{CO}(2-1)$ brightness temperatures along the radio jet axis of IC 5063. (d) Same as (c) but for the ratio of the $\mathrm{HCO}^{+}(4-3)$ and $\mathrm{CO}(2-1)$ brightness temperatures, (e) $\mathrm{CO}(2-1)$ spectrum of NGC 1266 . The blue and red shaded areas represent the outflow blue and red wing emission, respectively. (f) Map of the $\mathrm{CO}$ (2-1) blue and red wing emission defined in panel (e). (g) Profiles of $\mathrm{CO}(2-1)$ (top), $\mathrm{HCN}(1-0)$ (middle), and CS(2-1) (bottom) in NGC 1266. Dark blue shading indicates the outflow emission. Images reproduced with permission from (b) Morganti et al. (2015), (c, d) Oosterloo et al. (2017), copyright by ESO; and (a) Neff et al. (2015), (e, f) Alatalo et al. (2011), (g) Alatalo et al. (2015), copyright by AAS.

\subsubsection{IC 5063 and NGC 1266}

IC 5063 and NGC 1266 are two other well-studied low-power radio sources hosted by early-type hosts with prominent dust lanes and filaments. However, contrary to Cen A, both of these objects harbour clear neutral and molecular outflows within a kpc of their respective AGN. The case of the multi-phase jet-driven outflow in 
IC 5063 is well documented and is summarized in Figure 14b-d: large bulk and turbulent motion of the ionized, neutral, and molecular gas phases is present at the position of the bright north-western radio lobe $\left(1.8^{\prime \prime} \sim 520 \mathrm{pc}\right.$ from the nucleus), based on the detection at this location of a HI 21-cm absorption feature with a blue wing extending to about $-700 \mathrm{~km} \mathrm{~s}^{-1}$ (Morganti et al. 1998; Oosterloo et al. 2000) and broad emission lines with FWZI up to $\sim 1000 \mathrm{~km} \mathrm{~s}^{-1}$ from [O III] 5007 (Morganti et al. 2007), [Fe II] $1.644 \mu \mathrm{m}$ and $\mathrm{H}_{2} 2.128 \mu \mathrm{m}$ (Tadhunter et al. 2014; Dasyra et al. 2015), and $\mathrm{CO}$ and $\mathrm{HCO}^{+}$(Morganti et al. 2013; Dasyra et al. 2016; Oosterloo et al. 2017). More modest bulk and turbulent velocities of $\sim 100 \mathrm{~km} \mathrm{~s}^{-1}$ and $\sim 600 \mathrm{~km} \mathrm{~s}^{-1}$, respectively, are detected at the position of the weaker eastern radio lobe $\left(2.2^{\prime \prime} \sim 450\right.$ pc from the nucleus). Ionized, neutral, and molecular mass outflow rates of $\sim 0.1$, $\sim 35$, and $\gtrsim 12 \mathrm{M}_{\odot} \mathrm{yr}^{-1}$ are deduced from these data. The outflowing gas is of higher density ( $\gtrsim 2000 \mathrm{~cm}^{-3}$ for the ionized gas, from the [S II] ratio, and up to $\sim 10^{6} \mathrm{~cm}^{-3}$ in the molecular phase, based on the ratio of $\mathrm{HCO}^{+} / \mathrm{CO}(3-2)$ ) than the surrounding gas, as expected if the gas is compressed by the radio jet (e.g., Wagner et al. 2012). Note that the relative brightness of the ${ }^{12} \mathrm{CO}$ lines indicate that the outflowing molecular gas is optically thin, however (Dasyra et al. 2016; Oosterloo et al. 2017). Mukherjee et al. (2018b) have recently produced three-dimensional hydrodynamic simulations of a jet with powers $10^{44}-10^{45} \mathrm{erg} \mathrm{s}^{-1}$ propagating through the multi-phase gaseous disk of a galaxy that qualitatively reproduce many of the kinematic features observed in IC 5063, assuming that the jet turned on 0.24 Myr ago (Fig. 3b).

The kinematics of the neutral and molecular outflows in NGC 1266 are less extreme than those of IC 5063 (outflow velocities typically less than $250 \mathrm{~km} \mathrm{~s}^{-1}$; Fig. 14; Alatalo et al. 2011; Davis et al. 2012; Nyland et al. 2013), but the recent detection of broad wings in the HCN and CS spectra (Alatalo et al. 2015) indicates that the outflowing molecular gas is dense and optically thick, which boosts the inferred molecular mass outflow rate to $\sim 110 \mathrm{M}_{\odot} \mathrm{yr}^{-1}$, or $\sim 8 \times$ larger than the optically thin value reported in earlier papers. This large mass outflow rate and corresponding kinetic power of $\sim 3.4 \times 10^{45} \mathrm{erg} \mathrm{s}^{-1}$ are surprisingly high given the relatively modest radio power of the central VLBA-detected source in NGC 1266 (Nyland et al. 2013). The high brightness temperature $\left(\gtrsim 1.5 \times 10^{7} \mathrm{~K}\right)$ of this source is consistent with an AGN origin rather than a compact starburst. Indeed, star formation (total $\dot{M}_{*} \sim 0.87$ $\mathrm{M}_{\odot} \mathrm{yr}^{-1}$ ) seems to be suppressed by a factor $\sim 50$ given the amount of molecular gas in this object. Alatalo et al. (2015) have argued that the energy injected by the AGN stirs the host ISM and makes it more turbulent and less efficient at making new stars. Shock excitation has indeed been proposed to play a role in producing the line emission in the outflowing molecular gas (Glenn et al. 2015) and the fast (up to $\pm 900 \mathrm{~km}$ $\mathrm{s}^{-1}$ ) ionized outflow that extends to $\pm 4^{\prime \prime}$ ( $\pm 600 \mathrm{pc}$; Davis et al. 2012). Shocks could also conceivably be responsible for some of the X-ray emission seen on a similar scale (Alatalo et al. 2011).

\subsubsection{NGC 1377}

The most collimated molecular outflow discovered to date resides in the unassuming lenticular galaxy NGC 1377. It is nearby $(d=21 \mathrm{Mpc})$ and of moderate luminosity $\left(\log L_{\mathrm{IR}}=9.63\right.$; Roussel et al. 2006). Deep mid-infrared silicate absorption features 
imply that the nucleus is enshrouded by large masses of dust (e.g. Spoon et al. 2007). High resolution mm- and submm-wave studies suggest a nuclear $N\left(\mathrm{H}_{2}\right)$ of $\sim 10^{24}$ $\mathrm{cm}^{-2}$ (Aalto et al. 2016, 2017), but NGC 1377 is less obscured (on similar scales) than extreme CONs such as IC 860, Arp 220 or NGC 4418 (discussed in Sect. 5.5). NGC 1377 has a post-starburst optical spectrum which is likely a relic from a vigorous star formation event about a Gyr ago, possibly as a result of an interaction.

The molecular outflow was first detected in CO (2-1) at relatively low 0."6 (60 pc) resolution (Aalto et al. 2012) and appeared to be a young (1-2 Myr) biconic molecular flow. However, higher resolution (0.’2) ALMA observations revealed an extremely collimated, $150 \mathrm{pc}$ long jet-like structure in the CO 3-2 transition (Fig. 15), surrounded by a slower, wide-angle wind. There are so far no indications of an outflow in ionized or atomic gas-although a v-shaped dust lane can be seen on kpc scale in optical images. Interestingly, NGC 1377 is one of the most radio-quiet objects in the sky (e.g. Roussel et al. 2006; Costagliola et al. 2016) and there is so far no evidence of a radio jet that could be responsible for the high collimation of the high-velocity gas. Also, in contrast to molecular gas structures being carried out by radio-loud jets, the high-velocity molecular gas is found on the spine of the collimated flow. The $\mathrm{CO}(3-2)$ emission shows deviations from the symmetry axis, with velocity reversals, wiggles, and intensity variations on several size scales. The velocity reversals led to the suggestion that the molecular jet is precessing (Aalto et al. 2016). The velocity of the outflowing gas is difficult to determine but a lower limit is $v_{\text {out }}>240 \mathrm{~km} \mathrm{~s}^{-1}$ and may be as high as $850 \mathrm{~km} \mathrm{~s}^{-1}$ if the disk inclination is high. The total molecular mass in the collimated jet-like structure exceeds $2 \times 10^{6} M_{\odot}$. The CO emission is clumpy along the jet with a high velocity dispersion $(\sigma=40-90$ $\mathrm{km} \mathrm{s}^{-1}$ ) suggesting a highly turbulent medium and/or sideways ejection/expansion. The v-shaped minor axis structures (surrounding the molecular jet) are either part of a slower wide-angle wind, or material entrained by the molecular jet. In the latter case, the small $(150 \mathrm{pc})$ jet may potentially feed much of the mass into the kpc-scale bipolar structures.

The lack of star formation indicators (e.g no NIR $\mathrm{Pa} \alpha$ or $\mathrm{Br} \gamma$ and the extreme paucity of radio emission) have led to suggestions that NGC 1377 could be a nascent starburst (Roussel et al. 2006). However, the highly collimated, and relatively powerful high-velocity outflow, indicate that the molecular gas outflow is not driven by star formation. It may be powered by SMBH accretion - either by inefficient, hot ("radio-mode") accretion, or by effective accretion of cold gas ("quasar-mode"). In the former case the driving radio-jet is invisible, and in the latter case the molecular jet would represent an unexplored form of AGN feedback, where the high velocity gas is expelled in a collimated outflow and not a wide-angle wind.

It is also possible that the radio-quiet molecular jet of NGC 1377 represents accretion onto the nuclear disk (instead of onto the SMBH accretion disk) driving a magnetohydrodynamic (MHD) wind in a process reminiscent of that seen (on much smaller scales) in protostars. 


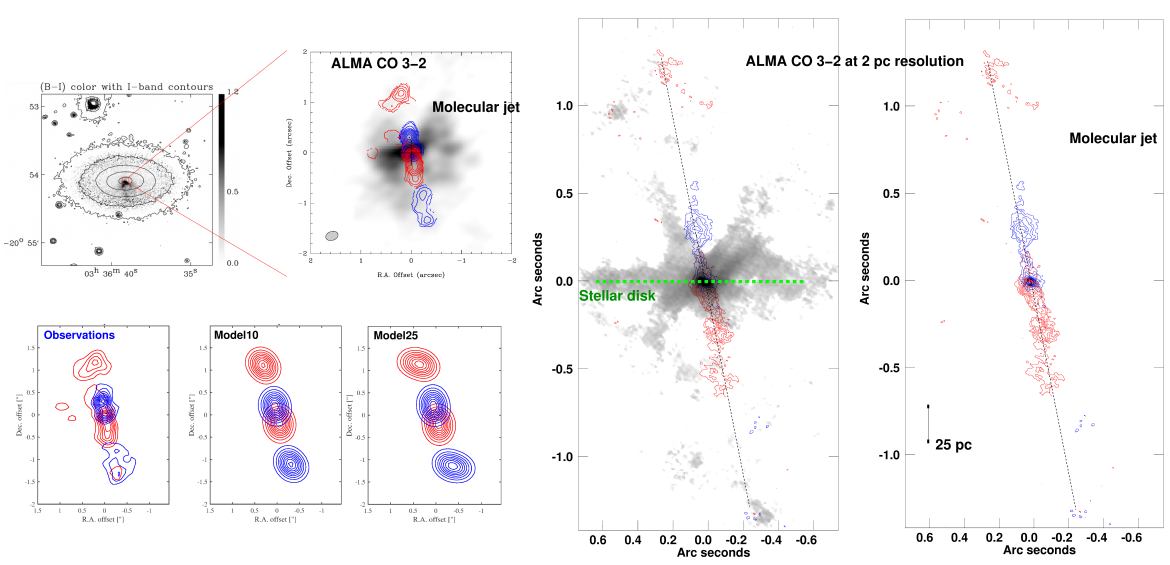

Fig. 15 Jetted molecular outflow in NGC 1377. Left: Optical (B-I) image (from Roussel et al. 2006) showing a southern outflow dust feature. Centre left: ALMA $20 \mathrm{pc}$ resolution CO (3-2) data where emission at systemic velocity $\left(0-60 \mathrm{~km} \mathrm{~s}^{-1}\right)$ is shown in gray scale and the high velocity (projected $80-160 \mathrm{~km}$ $\mathrm{s}^{-1}$ ) jet emission in red and blue contours. Bottom left panels: Models of the high velocity emission of a precessing jet. The left panel shows the observed data and the two right ones show models with different precession angle (Aalto et al. 2016). Right panels: High resolution (3 pc) ALMA CO (3-2) image (without the merged lower resolution data) showing the inner region and jet of NGC 1377 (Aalto et al. 2019, in prep.). Images reproduced with permission from Roussel et al. (2006), copyright by AAS; and Aalto et al. (2016), copyright by ESO.

\section{Cool Outflows in the Local $(z \lesssim 1)$ Universe}

Only very few galaxies have been studied at the level of detail as those discussed in Section 4. Many other galactic cool outflows have been identified using only one or a few tracers, and often with data that do not enable the extraction of detailed information, but only some basic outflows properties. The samples are also necessarily biased and incomplete. Yet, these several studies are useful to obtain statistical information of some of the basic properties of cool outflows as a function of other galactic properties, which can shed light on the driving mechanisms of cool outflows and their effects on galaxy evolution. This section summarizes the results on the local systems defined as $z \lesssim 1$. Section 6 discusses the results at higher redshifts.

\subsection{Neutral Atomic Gas Component}

Neutral atomic outflows have been detected in several $z \lesssim 1$ galaxies via "down-thebarrel" absorption-line spectroscopy of the host galaxies (including GRBs embedded in the hosts), direct emission-line imaging and 3D IFS of the cool outflows, and transverse absorption-line spectroscopy of background sources (quasars and galaxies) to probe the host CGM. The full panoply of diagnostic tools discussed in Section 3.3 has been brought to bear on the characterization of these outflows.

Historically, fast neutral-atomic outflows were first unambiguously detected in absorption in the spectra of quasars. Quasars with LOw-ionization Broad Absorption 
Lines or LoBALs are characterized by broad $\left(\gtrsim 1000 \mathrm{~km} \mathrm{~s}^{-1}\right.$ ) highly blueshifted ( $\lesssim$ $-1000 \mathrm{~km} \mathrm{~s}^{-1}$ ) absorption lines from $\mathrm{Mg} \mathrm{I}$ and $\mathrm{Mg}$ II. This class of quasars is rare, accounting for only $\sim 1.3 \%$ of all quasars (Trump et al. 2006). LoBALs with excited states of Fe II or Fe III absorption ("FeLoBALs" such as Mrk 231; Sect. 4.4) are even less common ( $\sim 0.3 \%$; Trump et al. 2006). The sizes of these low-ionization outflows, derived using Eq. (40) in the few $z \lesssim 1$ objects where the electron density can be constrained, span a broad range from a few pc to a few kpc (Moe et al. 2009; Dunn et al. 2010; Bautista et al. 2010; Aoki et al. 2011; Veilleux et al. 2016). These fast nuclear winds may be the driving force behind the outflows seen on larger scales in these same objects (Sect. 2.2.1 and 5.4).

Ironically, few neutral-atomic outflows have been detected using H I $21 \mathrm{~cm}$ (Sect. 3.3.1), until recently (see review by Morganti and Oosterloo 2018). While this largely reflects the technical limitations of early-generation radio facilities, H I photoionization also plays a role behind the low detection rate of $\mathrm{H}$ I 21-cm absorption line in powerful AGN with ionization rates $Q_{\mathrm{HI}} \gtrsim 3 \times 10^{56} \mathrm{~s}^{-1}$ (e.g., Curran et al. 2016, 2019; Aditya and Kanekar 2018). In contrast, down-the-barrel ground-based observations of absorption lines from neutral-atomic tracers such as Na I D 5890, 5896, Mg II 2796, 2803, and Fe II 2586, 2600 against the host galaxy light (Sect. 3.3.3, 3.3.4, and 3.3.5) have been extremely successful at detecting cool outflows in gasrich $z \lesssim 1$ galaxies. Fast $\mathrm{Na}$ I outflows with inferred hydrogen column densities $N_{\mathrm{H}} \simeq 10^{20}-10^{22} \mathrm{~cm}^{-2}$ (based on Eq. (39)) are ubiquitous in powerful dusty starburst and active galaxies, where dust in the hosts and within the outflowing gas shields $\mathrm{Na}$ I $(5.1 \mathrm{eV})$ against the $\lesssim 2432 \AA$ radiation emitted by the starbursts and AGN (e.g., nearby U/LIRGs, Heckman et al. 2000; Rupke et al. 2002, 2005b,c,a; Martin 2005, 2006; Cazzoli et al. 2016). The detection rate (and velocities) of $\mathrm{Na}$ I outflows drops precipitously with decreasing dust content $A_{V}$, SFR, SFR per unit area, AGN power, and stellar mass, averaging a value $\lesssim 1 \%$ among the general population of opticallyselected star-forming and active galaxies in SDSS (e.g., Sarzi et al. 2016; Bae et al. 2017; Concas et al. 2019; Nedelchev et al. 2019; Roberts-Borsani and Saintonge 2019), in MaNGA-selected star-forming galaxies (Roberts-Borsani et al. 2020), as well as in IR-faint quasars (Krug et al. 2010). Na I outflows in non-ULIRGs show a dependence on galaxy disk inclination that indicates a preference for $\mathrm{Na}$ I outflows to align along the minor axis of the host galaxy disk (Fig. 16; e.g., Chen et al. 2010; Concas et al. 2019; Roberts-Borsani and Saintonge 2019). Recent Na I IFS observations of local ULIRGs and IR-bright quasars hint at a similar alignment of the neutral outflowing gas along the minor axis of the underlying rotating gas structures on kpc scales, although these outflows clearly subtend a wider angle and are more irregular than those found in systems of lower SFRs and AGN powers (Rupke and Veilleux 2013b; Rupke et al. 2017).

Large ground-based optical spectroscopic surveys in the past decade have extended these down-the-barrel studies of neutral-atomic outflows to higher redshifts using the Mg II 2796, 2803, Fe II 2586, 2600, and Mg I 2853 absorption features redshifted into the optical band (e.g., Weiner et al. 2009; Rubin et al. 2010; Coil et al. 2011; Erb et al. 2012; Kornei et al. 2012, 2013; Martin et al. 2012; Bordoloi et al. 2014a; Rubin et al. 2014; Zhu et al. 2015; Heintz et al. 2018, using GRBs). Mg II outflows with typical inferred hydrogen column densities $N_{\mathrm{H}} \gtrsim 10^{19} \mathrm{~cm}^{-2}(\mathrm{Mg}$ II is 

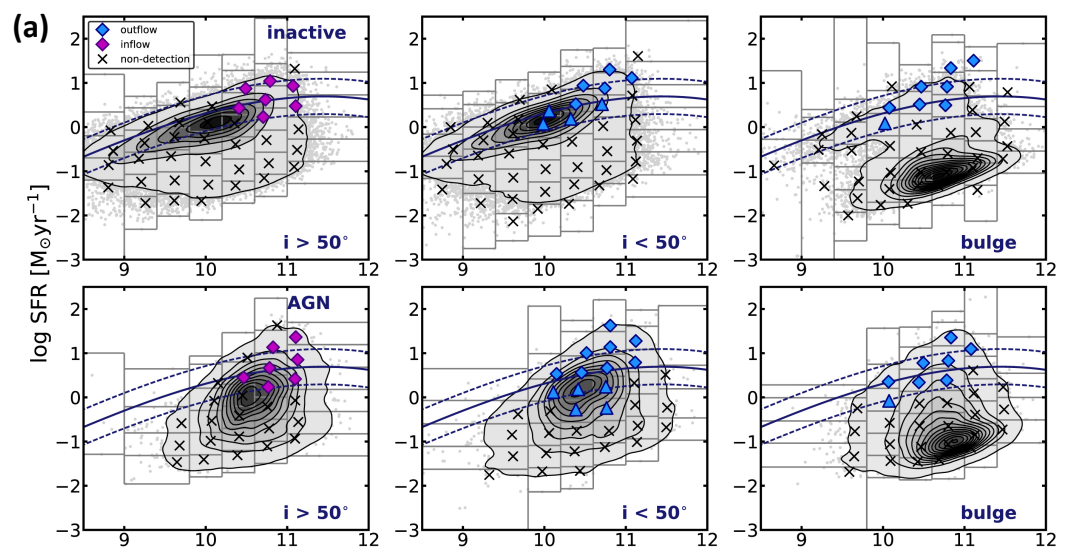

$\log M_{*}\left[M_{\odot}\right]$
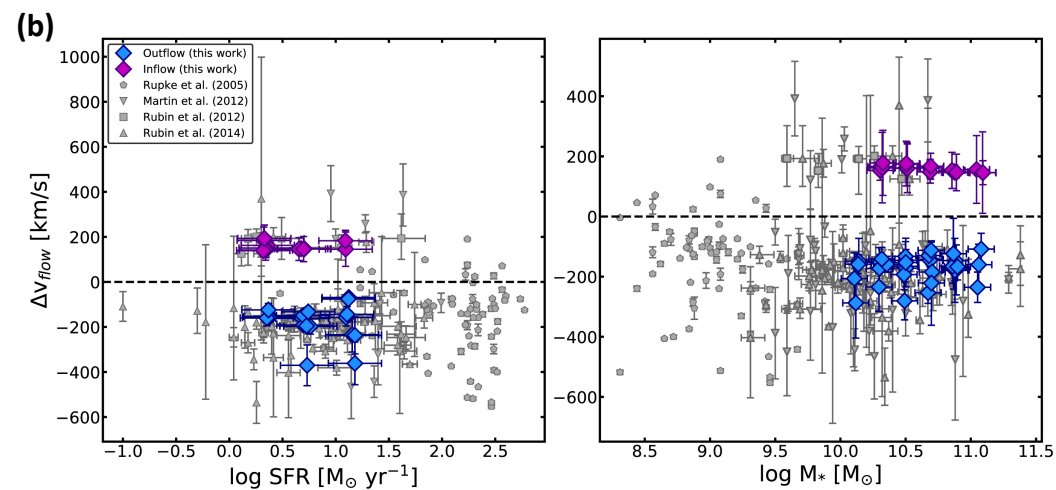

Fig. 16 Statistical properties of nearby neutral-atomic outflows based on the Na I D absorption doublet. (a) Detections of inflows and outflows across the SFR $-M_{*}$ plane for disk galaxies (left and middle panels are for different inclinations) and bulge galaxies (right panels). The top row shows inactive galaxies while the bottom row shows AGN hosts. (b) Average inflow and outflow velocities as a function of SFR (left panel) and $M_{*}$ (right panel). Images reproduced with permission from Roberts-Borsani and Saintonge (2019), copyright by the authors.

saturated when $\mathrm{EW}_{r} \gtrsim 1 \AA$ so these columns are lower limits) are detected in most $(\gtrsim 60 \%) z \sim 0.5-1.5$ star-forming galaxies with $\mathrm{SFR} \gtrsim 1 \mathrm{M}_{\odot} \mathrm{yr}^{-1}$, particularly faceon galaxies $(\sim 90 \%)$, implying ubiquitous biconical outflows with opening angle $\sim$ $100^{\circ}$ (Rubin et al. 2014). The wind maximum velocities (typically $\sim 200-400 \mathrm{~km}$ $\mathrm{s}^{-1}$ ) and equivalent widths correlate only weakly with galaxy stellar mass and SFR (Fig. 17, Weiner et al. 2009; Rubin et al. 2014). "Relic" outflows with typical velocities $\sim 200 \mathrm{~km} \mathrm{~s}^{-1}$ are often seen in post-starburst galaxies (Coil et al. 2011; Yesuf et al. 2017), although in exceptional cases, possibly due to recent quasar activity or extremely compact optically obscured starbursts, these winds reach velocities in excess of $\sim 1000 \mathrm{~km} \mathrm{~s}^{-1}$ (Tremonti et al. 2007; Diamond-Stanic et al. 2012; Geach et al. 2014; Sell et al. 2014; Maltby et al. 2019). In general, low-luminosity AGN do not 
have a strong influence on the velocities and equivalent widths of these winds (Coil et al. 2011; Yesuf et al. 2017).

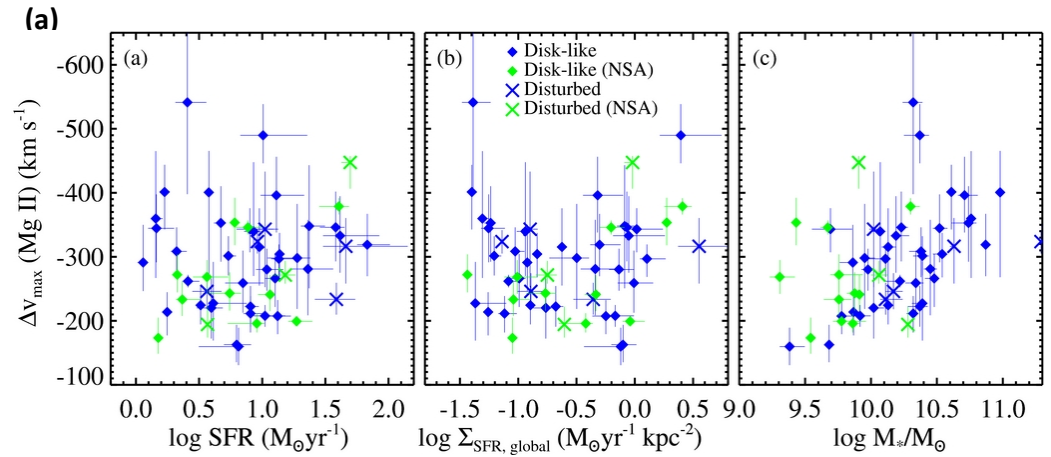

(b)
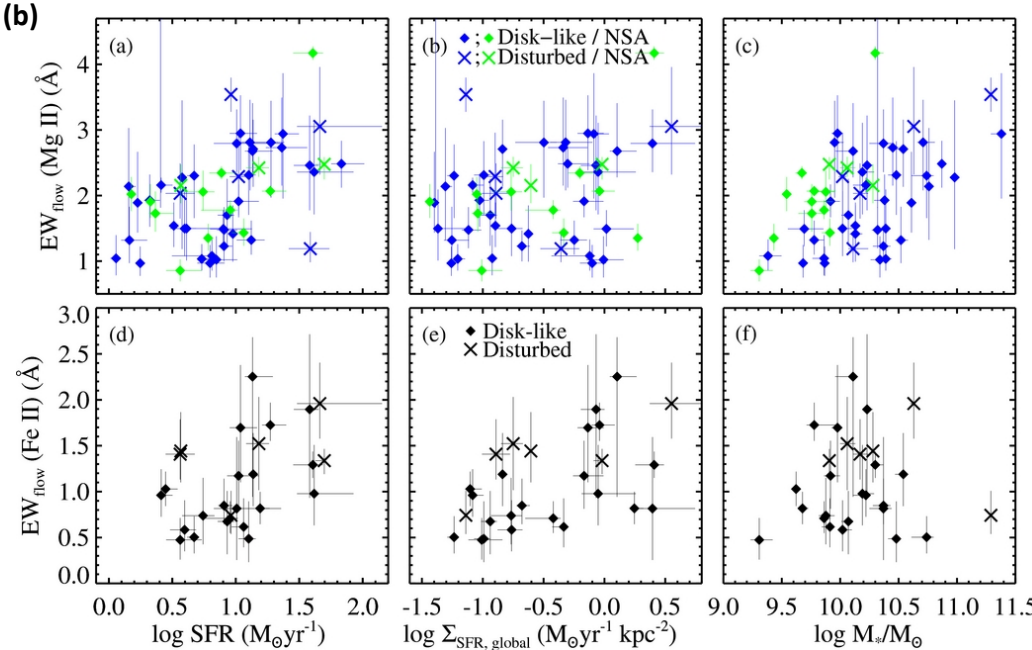

Fig. 17 Statistical properties of neutral-atomic outflows based on the NUV Mg II and Fe II absorption lines. (a) Maximum outflow velocities as a function of SFR, SFR surface density, and stellar mass. (b) Equivalent widths of Mg II (top panels) and Fe II (bottom panels) as a function of the same quantities as in (a). Images reproduced with permission from Rubin et al. (2014), copyright by AAS.

The excellent FUV sensitivity of the Cosmic Origins Spectrograph (COS) onboard HST has allowed to study in detail, albeit with limited spatial information, the cool neutral-atomic and warm ionized gas phases of outflows in some of the nearest and UV-brightest star-forming galaxies (e.g., Heckman et al. 2011, 2015; Heckman and Borthakur 2016; Chisholm et al. 2015, 2016a,b, 2017, 2018) and even in a few infrared-bright systems (Leitherer et al. 2013; Martin et al. 2015; Veilleux et al. 2016). Chisholm et al. (2015) find weak (3.0-3.5 $\sigma$ ) correlations between the Si II-based outflow velocities and SFR $\left(v_{\text {out }} \propto \dot{M}_{*}^{0.08-0.22}\right)$, the stellar mass $\left(\propto M_{*}^{0.12-0.20}\right)$, and the circular velocity $\left(\propto v_{\text {circ }}^{0.44-0.87}\right)$, where the index depends on whether the maximum 
or centroid velocity is used as the outflow velocity (Fig. 18a). In contrast, Heckman et al. (2015) and Heckman and Borthakur (2016) find stronger correlations between the maximum velocity and SFR ( $v_{\text {out }} \propto \dot{M}_{*}^{0.32}$ ), and the star formation rate surface density $\Sigma_{\mathrm{SFR}}$ (Fig. 18b), consistent with momentum-driven winds where the momentum comes from a combination of ram pressure from the wind, radiation pressure, and cosmic rays (Sec. 2.2). The origin of the apparent discrepancy between these kinematic studies is not clear, but is probably due to more than just one factor. The results of Heckman et al. (2015) and Heckman and Borthakur (2016) are based on Si III $1206\left(\mathrm{IP}^{+}=16.3 \mathrm{eV}\right)$ rather than Si II $1260\left(\mathrm{IP}^{0}=8.15 \mathrm{eV}\right)$, so they trace the ionized component of these outflows rather than the neutral-atomic component. However, this cannot fully explain the apparent discrepancy between these studies since both Heckman et al. (2015) and Chisholm et al. (2015) find that Si IV, Si III, Si II, and O I have virtually the same profiles and are thus co-moving within a single outflowing structure. The limited number $(\$ 50)$ of objects examined in these studies no doubt adds noise to possible underlying relations. Sample selection may also be critical. While there is considerable overlap between the two samples, it is also clear that the addition of the extreme starbursts from Diamond-Stanic et al. (2012) and Sell et al. (2014) in the study by Heckman and Borthakur (2016) extends the range of SFR and $\Sigma_{\mathrm{SFR}}$ over the previous studies and therefore provides more leverage for the fits. This comparison also raises a cautionary flag that the wide-spread practice of reducing the velocity fields of these complex three-dimensional multi-phase outflows to a single parameter $\left(v_{50}\right.$ or $\left.v_{\max }\right)$ in a single tracer is bound to introduce considerable scatter in the scaling relationship with the host galaxy properties (see Table 1 of Rupke 2018, for a summary).

To go beyond a discussion of the kinetics of cool neutral-atomic outflow and address their dynamical properties requires a measure of their physical extents (Sec. 3.2). The sizes of the outflows studied in the above HST data $(r \lesssim 1 \mathrm{kpc})$ are derived indirectly from detailed modeling of the photoionization conditions in the outflowing material, carefully taking into account object-by-object variations in the metallicity and ionization corrections (e.g., Chisholm et al. 2016a,b). In contrast, direct size measurements exist for a significant fraction of the cool neutral-atomic outflows based on the Na I absorption line; they typically extend to a few $\mathrm{kpc}$, with a range from $\lesssim$ $1 \mathrm{kpc}$ to $\sim 15 \mathrm{kpc}$ (Rupke et al. 2005c; Martin 2006; Rupke et al. 2017; RobertsBorsani et al. 2020). These measurements should be considered lower limits since they are limited by the sensitivity of optical spectrographs to detect this absorption line against the faint galaxy continuum at large $r$. This has been made clear by the detection in a few objects of redshifted resonant $\mathrm{Na}$ I emission well beyond the scale where blueshifted Na I absorption is detected (Fig. 19a; Phillips 1993; Rupke and Veilleux 2015). Scattered resonant and non-resonant line emission has also proven to be very useful to constrain the size of the Mg II and Fe II outflows, which would otherwise remain largely unconstrained from the absorption-line studies. The Mg II and $\mathrm{Fe} \mathrm{II}^{*}$ emission line sizes derived from stacked long-slit composite spectra range from a few kpc up to $20 \mathrm{kpc}$ (Rubin et al. 2011; Erb et al. 2012; Martin et al. 2013), although recent direct imaging and 3D IFS data indicate that most of the line emission appears to come from $\lesssim 4-10 \mathrm{kpc}$ (Fig. 19b; Finley et al. 2017; Rickards Vaught et al. 2019), and may be localized and strongly influenced by the nearest star-forming 
(a)

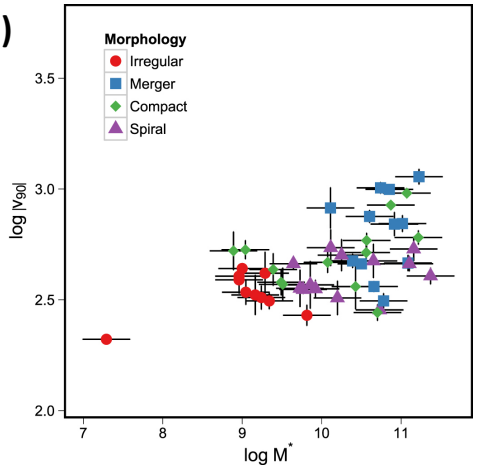

(b)

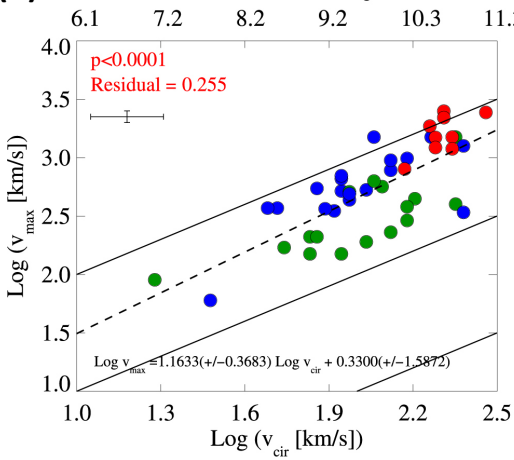

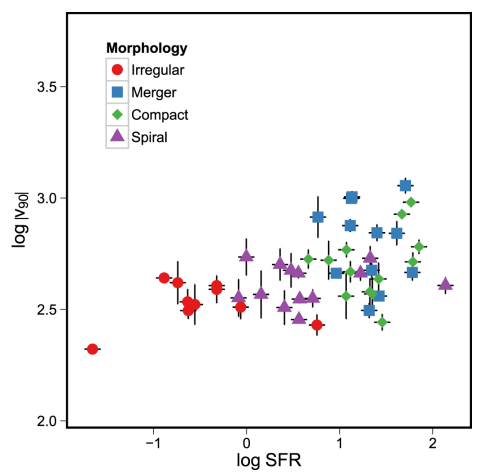

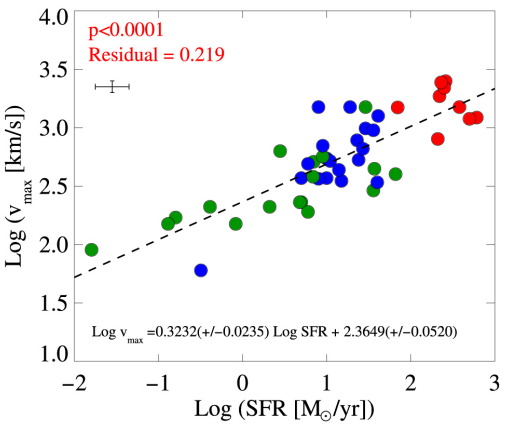

Fig. 18 Statistical properties of nearby low-ionization outflows based on HST spectra. (a) 90-percentile Si II velocities as function of stellar mass and SFR, (b) Maximum Si III velocities as a function of the circular velocity and SFR. Images reproduced with permission from (a) Chisholm et al. (2015), (b) Heckman and Borthakur (2016), copyright by AAS.

clump, based on IFS of a few lensed systems at $z \sim 1$ (Bordoloi et al. 2016; Karman et al. 2016, most of the work on lensed systems has been done at higher redshifts; see Sec. 6.2). Direct size measurements of neutral-atomic outflows also exist for a few nearby sources mapped in the [C II] $158 \mu \mathrm{m}$ line emission using Herschel or SOFIA (Contursi et al. 2013; Kreckel et al. 2014; Appleton et al. 2018, Stone et al. 2019, in prep.). All of these direct size measurements should be considered lower limits since they are severely limited by the sensitivity of the observations. The recent detection of Mg II-line emitting material out to $20 \mathrm{kpc}$ in Makani, a compact starburst-dominated galaxy at $z=0.459$ mapped with deep KCWI IFS data (Fig. 19; Rupke et al. 2019), underscores the need for deeper observations.

Transverse absorption-line studies of $z \lesssim 1$ galaxies using background quasars and galaxies as probes of the CGM have provided additional constraints on the sizes of cool neutral-atomic outflows (see review by Tumlinson et al. 2017). Studies of individual star-forming galaxies (Kacprzak et al. 2012; Martin et al. 2019) and stacked spectra (Bordoloi et al. 2011; Ménard et al. 2011; Lan et al. 2014; Lan and Mo 2018; Lan 2019) have shown that the strongest $E W_{r} \gtrsim 0.3 \AA \mathrm{Mg}$ II absorbers with 
(a)

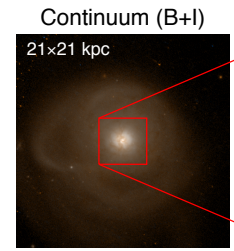

(b)

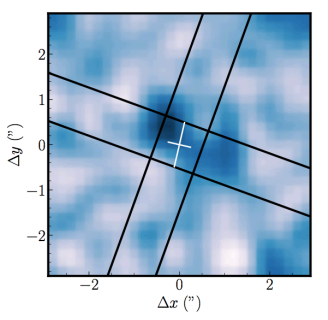

Dust (B-I)
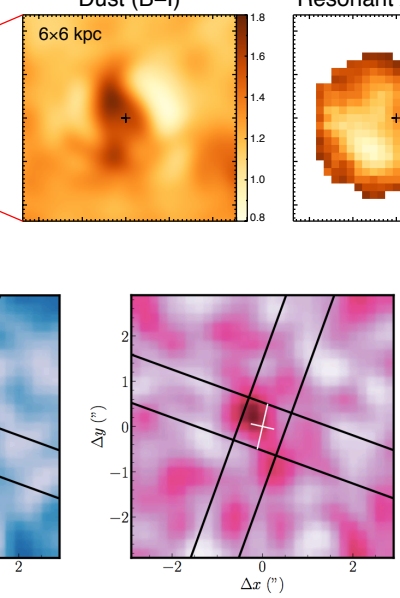

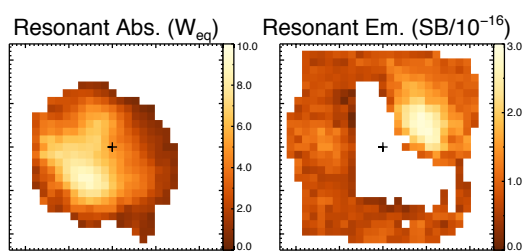

(c)

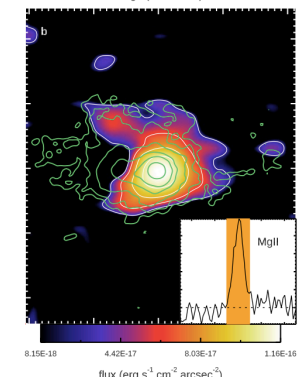

Fig. 19 Nearby neutral-atomic outflows in line emission. (a) Resonant Na I line emission (rightmost panel) from the redshifted outflow component in a nearby ULIRG, compared with the Na I absorption and dust distributions on the same scale. (b) Non-resonant $\mathrm{Fe} \mathrm{II}^{*}$ line emission from the blueshifted (left) and redshifted (right) outflow components in a $z \simeq 1.24$ galaxy. (c) Resonant $\mathrm{Mg}$ II line emission in $z=0.46$ Makani. Images reproduced with permission from Rupke and Veilleux (2015), copyright by AAS; and Finley et al. (2017), copyright by ESO; and Rupke et al. (2019), copyright by the authors.

$N_{\mathrm{H}} \gtrsim 3 \times 10^{18} \mathrm{~cm}^{-2}$ lie within $\sim 50-80 \mathrm{kpc}\left(\lesssim 0.25-0.40 R_{\mathrm{vir}}\right)$ and are more prevalent along the minor axis of disk galaxies, as expected if they trace biconical cool outflows emerging from the disk (Fig. 20). A similar result was found by Zhu and Ménard (2013) using strong Ca II H+K absorbers. The remaining strong Mg II absorbers seem largely aligned with the major axis of the disk and in co-rotation with the disk (Bouché et al. 2012; Nielsen et al. 2015; Martin et al. 2019; Zabl et al. 2019). The excess of strong Mg II absorbers within $\sim 50-80 \mathrm{kpc}$ may be even more pronounced among quasars, especially luminous ones, although $\mathrm{Mg}$ II is rarely detected down-the-barrel in these quasars suggesting that the nearest Mg II clouds have been photoionized by the central AGN (Farina et al. 2014; Johnson et al. 2015). As discussed in Sect. 6.2, a similar deficit is seen in quasars at higher redshifts, based on C II absorbers (Prochaska et al. 2014; Lau et al. 2016).

Passive galaxies, on the other hand, show a lower-though non-zero-rate of incidence of strong Mg II absorbers within $\sim 50 \mathrm{kpc}$ than star-forming galaxies (Fig. 20ab; Lan et al. 2014; Lan and Mo 2018; Lan 2019), while the rate of incidence of H I absorption in general, and that of strong Mg II absorption on scales greater than 100 $\mathrm{kpc}$, are virtually the same for both galaxy populations (Thom et al. 2012; Lan et al. 2014; Keeney et al. 2017; Lan and Mo 2018). Similarly, C II and Si II absorption from the CGM around local dwarfs with modest SFR is much less common than in massive star-forming galaxies (Prochaska et al. 2014; Johnson et al. 2017). This last result seems at odds with model expectations of increasing mass-loading (and metalloading) factors with decreasing stellar masses (Muratov et al. 2015, 2017), and the 

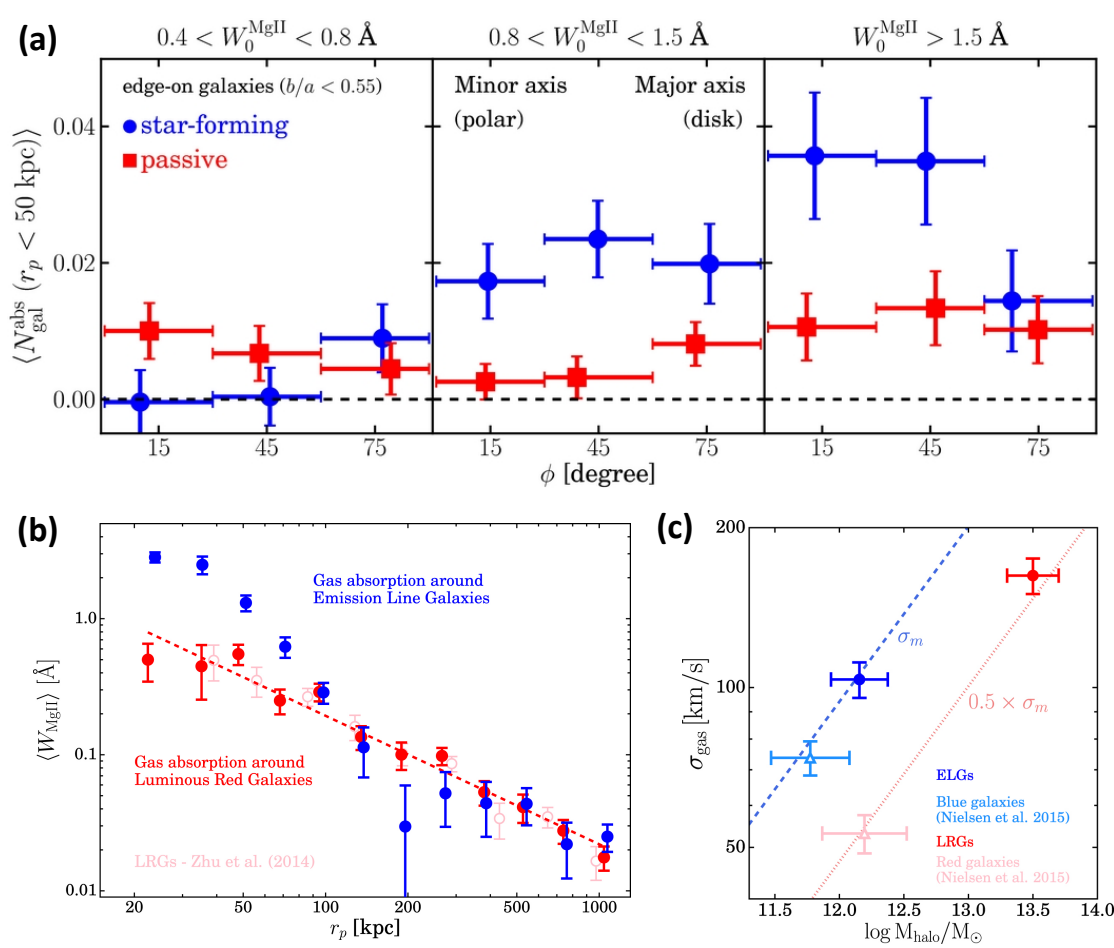

Fig. 20 Statistical properties of NUV Mg II absorbers. (a) Frequency of occurrence as a function of position angle with respect to the disk minor axis, for star-forming (blue) and passive (red) galaxies. (b) Mg II equivalent width as a function of the distance from emission-line galaxies (blue) and luminous red galaxies (red). (c) Line-of-sight Mg II velocity dispersion as a function of halo mass and galaxy types (color-coded). Images reproduced with permission from (a) Lan et al. (2014) and (b, c) Lan and Mo (2018), copyright by AAS.

recent observations that support these predictions (Chisholm et al. 2017), unless the CGM of these dwarf galaxies are dominated by unexpectedly high ionization states like C IV and O VI (Bordoloi et al. 2014b), contrary to the results of Mathes et al. (2014). The difference between passive and actively star-forming galaxies is also reflected in (1) the velocity distribution of the clouds in the absorption profiles, with the blue star-forming galaxies showing a larger velocity dispersion than red galaxies of the same masses, consistent with outflows in the star-forming galaxies (Fig. 20c; e.g., Nielsen et al. 2016; Lan and Mo 2018), and (2) the radial profiles of the Fe II/Mg II ratio, which indicate a substantial contribution from Type Ia SN enrichment in the inner halos of passive galaxies, but dominant enrichment from core-collapse $\mathrm{SNe}$ throughout the halos of star-forming galaxies and in the outer halo of passive galaxies (Zahedy et al. 2016). The well-known correlation between velocity width and metallicity in DLAs with $N_{\mathrm{H}}>10^{20.3} \mathrm{~cm}^{-2}$ has been shown to extend down to sub-DLAs with $N_{\mathrm{H}}=10^{19}-10^{20.3} \mathrm{~cm}^{-2}$ (Som et al. 2015; Quiret et al. 2016). This correlation has traditionally been interpreted as a mass-metallicity relation of the DLA galaxy 
hosts, but in the present context it may instead reflect a connection between the wind kinematics and the metallicity of the outflowing material.

Overall, down-the-barrel observations of cool outflows and transverse absorption line studies of the cool CGM paint a picture where stellar feedback is capable of driving cool neutral-atomic outflows with radial sub-virial velocities of $\sim 100-200$ $\mathrm{km} \mathrm{s}^{-1}$ out to $50-80 \mathrm{kpc}\left(0.25-0.40 R_{\text {vir }}\right)$ into the halo, expelling cool gas out of the central regions of the galaxies at rates that scale loosely with $\dot{M}_{*}$ and $\Sigma_{\mathrm{SFR}}$. It is also clear that the presence of a powerful quasar provides a significant boost in velocity, momentum, and energy over and above those provided by the stellar processes, but quasars also destroy the neutral gas in its proximity, reducing the rate of incidence of line-of-sight neutral-atomic outflows unless the gas is shielded by dust in the host or embedded in the outflow itself, as it is the case in Na I outflows. AGN of lower luminosities do not significantly impact the kinematics of the neutral-atomic gas in the host galaxies (this statement also seems to apply in general to the ionized phase of these systems, Wylezalek et al. 2019), although heating by these AGN may still significantly reduce the ability of the cool gas to form stars (see also Sect. 5.2).

The cool outflowing material no doubt evolves on its way out of the galaxy, eventually decelerating and becoming more diffuse on average as it reaches CGM scales. The inferred hydrogen column densities of the cool clouds cited above $\left(N_{\mathrm{H}} \simeq 10^{20}\right.$ $10^{22} \mathrm{~cm}^{-2}$ at the base of the outflows based on $\mathrm{Na}$ I D measurements and $N_{\mathrm{H}} \simeq 10^{18}$ $10^{20} \mathrm{~cm}^{-2}$ in the strongest $\mathrm{Mg}$ II absorbers in the CGM) are uncertain and modeldependent. The results depend sensitively on the ionizing field (is the starburst and/or AGN contributing to the ionization in addition to the UV background radiation field?) and the cloud structure (is the gas along each line of sight characterized by a single density or a range of densities; e.g., Werk et al. 2014; Stern et al. 2016). The inferred volume densities of these clouds are equally uncertain, although it is clear that they span a broad range of values: $n_{\mathrm{H}} \simeq 10^{-3}-0.1 \mathrm{~cm}^{-3}$ for the Mg II CGM clouds (Prochaska et al. 2011; Kacprzak et al. 2014; Werk et al. 2014; Stern et al. 2016; Thompson et al. 2016; Zahedy et al. 2019), while $n_{\mathrm{H}} \simeq 0.1-10^{2} \mathrm{~cm}^{-3}$ in the inner Mg II and Na I outflows (Prochaska et al. 2011; Tanner et al. 2016, 2017). The inferred cloud thickness of the Mg II absorbers in the CGM ranges from $\lesssim 10$ pc to $\sim 1 \mathrm{kpc}$ (Stern et al. 2016; Zahedy et al. 2019), while their coherence length scale across the sky, based on comparisons between results on QSO-galaxy and galaxygalaxy pairs (Rubin et al. 2018a,b), exceed $\sim 1.9 \mathrm{kpc}$, reflecting the scale over which the number and velocity dispersion of these structures are spatially correlated. In the few cases where it is measured reliably, the metallicity of the outflow on $\lesssim \mathrm{kpc}$ scales is roughly solar, regardless of the host stellar mass (Chisholm et al. 2018). The metal loading factor inversely scales with stellar mass, consistent with models where outflows help shape the galaxy mass-metallicity relation (Mac Low and Ferrara 1999; Muratov et al. 2015, 2017; Emerick et al. 2018; Forbes et al. 2019; Maiolino and Mannucci 2019, and references therein).The metallicity of $\mathrm{Mg}$ II absorbers on CGM scales are typically $1 / 10$ that of the hosts, although there is significant scatter (Kacprzak et al. 2014, 2019). No doubt, complex mixing between the outflowing material and the accreting and recycled gas is taking place in the CGM, and can account for some of the large point-to-point metallicity variations (Pointon et al. 2019). 


\subsection{Molecular Gas Component}

The detection of P-Cygni profiles (or simply blueshifted absorption) of the $\mathrm{OH}$ transitions of $79 \mu \mathrm{m}$ or $119 \mu \mathrm{m}$ (as discussed in Section 3.4.4), which identified the first cool molecular outflow (in Mrk 231, Sect. 4.4, Fischer et al. 2010), has now been extended to $\sim 50$ galaxies thanks to Herschel spectroscopy (Sturm et al. 2011; Veilleux et al. 2013a; Spoon et al. 2013; Stone et al. 2016). These samples are generally mostly restricted to (U)LIRGs, given the requirement of having a strong far-IR source in order to get enough signal to noise on the continuum to properly detect these features in absorption. Studies involving the detection of multiple molecular species and multi-transition absorption features, which enable a more detailed modelling of the outflow, are still restricted to about a dozen galaxies (González-Alfonso et al. 2012, 2013, 2014, 2017b). In these cases detailed information on the outflow rate, energetics and size of the outflow can be inferred (although the abundances of the various molecular species are still difficult to constrain properly despite recent progress; Stone et al. 2018). Interestingly, the joint analysis of multiple transitions has shown a stratification of the outflow with the inner regions (with sizes ranging from a few pc to $\sim 100 \mathrm{pc}$ ) having higher temperatures (up to $400 \mathrm{~K}$ ) and high column densities (up to $\mathrm{N}_{\mathrm{H}}>10^{25} \mathrm{~cm}^{-2}$ ) and an outer, cooler $(<100 \mathrm{~K}$ ) and more rarefied $\left(\mathrm{N}_{\mathrm{H}} \sim 10^{22}-10^{23} \mathrm{~cm}^{-2}\right)$ envelope, distributed on scales of several hundred parsec. One should however take into account that the far-IR absorption features tend to preferentially probe the inner, more IR-luminous regions of the galaxy (given by the requirement of having a strong background far-IR source), hence more extended component of outflows may be missed. For all other cases, for which only one or a few transitions are available, generally the molecular absorption only provides some basic information on the outflow velocity distribution.

The identification of molecular outflows through the detection and mapping of $\mathrm{CO}$ transitions associated with high velocity gas has now been possible for more than fifty local galaxies, especially in recent years thanks to ALMA enabling high sensitivity and high angular resolution observations (see e.g. compilations in Cicone et al. 2014; Fiore et al. 2017; Fluetsch et al. 2019; Lutz et al. 2019). Some of these observations are very detailed and reveal very clumpy structures (e.g. Pereira-Santaella et al. 2016, 2018; Cicone et al. 2019) and complex morphologies on different scales, often difficult to describe with simple models (Combes et al. 2014; Tsai et al. 2012; Sakamoto et al. 2014; García-Burillo et al. 2014, 2016; Gallimore et al. 2016; Aalto et al. 2016; Pereira-Santaella et al. 2016; Falstad et al. 2017; Pereira-Santaella et al. 2018; Herrera-Camus et al. 2019a; Treister et al. 2020). However, the bulk of these observations are often still limited to the detection of high velocity wings of the CO transitions, and only marginally resolved. This makes it difficult to properly characterize the outflow properties, such as the outflow rate, kinetic power and momentum rate, as the direct determination of the outflow extent is an important piece of information to constrain these quantities (Sec. 3.2). One should also keep in mind that these studies obviously suffer from biases. In particular, weak and low velocity outflows tend to be missed, as the $\mathrm{CO}$ emission associated with these motions are difficult to disentangle from the $\mathrm{CO}$ emission coming from the galactic disk, unless detailed, high angular resolution mapping is available which enable modelling (and subtract- 
ing) the galactic rotation curve (e.g. Zschaechner et al. 2016; Pereira-Santaella et al. 2016; Gallimore et al. 2016).

Generally, the outflow is traced in only one CO transition. In those few cases for which multiple transitions are observed, these are typically consistent with an excitation similar to that observed in the host galaxy (e.g. Cicone et al. 2012; Feruglio et al. 2015), although (as discussed in Sec. 4.4) there are also cases where the CO excitation in the outflow is remarkably different than typically observed in galactic disks, and in a few cases the very high $\mathrm{CO}$ excitation suggests an optically thin molecular medium (Dasyra et al. 2016).

The detection of additional molecular species in the outflow is even rarer. However, it is often found that dense gas molecular tracers (such as $\mathrm{HCN}, \mathrm{HCO}^{+}, \mathrm{CN}$ ) are enhanced in galactic outflows (Aalto et al. 2012; García-Burillo et al. 2014; Aalto et al. 2015a; Walter et al. 2017; Falstad et al. 2018; Barcos-Muñoz et al. 2018; Harada 2018; Michiyama et al. 2018; Impellizzeri et al. 2019). Evidence that the outflowing gas is systematically characterized by higher densities than in the host galaxy is also found for the warm ionized component, as highlighted by various studies of the density-sensitive nebular diagnostics, both in AGN and SF-driven winds (Perna et al. 2017; Mingozzi et al. 2019; Hinkle et al. 2019, Gallagher et al. 2019, in prep., Fluetsch et al. 2019, in prep.). These results suggest that gas compression makes the dense phase of the molecular gas more prevalent in outflows than in galactic disks (as suggested by some models, Zubovas and King 2014; Richings and Faucher-Giguère $2018 \mathrm{~b}$ ), or that the more diffuse molecular phase is more easily evaporated and destroyed (Scannapieco 2017; Decataldo et al. 2017) or both phenomena are at work. Furthermore, recent models have suggested that massive molecular clouds in the outflows are self-gravitating and may evolve into dense cores (Decataldo et al. 2019).

The range of $\mathrm{CO}$ excitation properties and the range of dense-to-diffuse molecular gas ratio suggests that the $\mathrm{CO}-$ to- $\mathrm{H}_{2}$ conversion factor in the outflow is also subject to variations from case to case, which adds uncertainty to the determination of the outflow properties. Those few outflows with multiple transitions available and also observed in other species, suggest a broad range of conversion factors, spanning about an order of magnitude, ranging from $\alpha_{C O} \sim 2 M_{\odot}\left(\mathrm{K} \mathrm{km} \mathrm{s}^{-1} \mathrm{pc}^{2}\right)^{-1}$ to $\alpha_{C O} \sim 0.3 M_{\odot}\left(\mathrm{K} \mathrm{km} \mathrm{s}^{-1} \mathrm{pc}^{2}\right)^{-1}$ (Weiß et al. 2001; Dasyra et al. 2016). The vast majority of studies cannot really constrain $\alpha_{C O}$, hence a fixed $\alpha_{C O}$ is generally assumed. In Aalto (2015), the impact of self-gravitating versus non-self gravitating dense gas on the mass estimate is discussed.

Within this context, as already mentioned in Section 3.4.4, molecular outflows have also started to be traced by exploiting sensitive observations of [C I] (e.g. Cicone et al. 2018b), which (although locally these transitions are in a frequency range more difficult to observe) is arguably a better tracer of the molecular gas. In particular, the [C I]-to- $\mathrm{H}_{2}$ conversion is considered less affected by environmental conditions and scales only linearly with metallicity (as opposite to $\alpha_{C O}$ which has a debated and not fully understood super-linear dependence on metallicity). As already mentioned, ALMA [C I] observations of the study case NGC 6240 have confirmed the presence of a very massive fast molecular outflows extending on scales of several kpc scale 
(Cicone et al. 2018b). The same observations have suggested that the CO-to- $\mathrm{H}_{2}$ conversion factor in the outflow is as high as $\alpha_{C O} \sim 2 M_{\odot}\left(\mathrm{K} \mathrm{km} \mathrm{s}^{-1} \mathrm{pc}^{2}\right)^{-1}$.

Despite all of the biases and uncertainties discussed above, statistical studies of molecular outflows in local galaxies have provided interesting information on the scaling relations between the properties of molecular outflows and those of their host galaxies.

Far-IR OH P-cygni profiles have provided the clearest identifications of outflows and clear statistics on the outflow velocities, generally avoiding ambiguity with other types of non-virial motions in studies based on transitions in emission. However, geometrical effects and orientation have certainly prevented the detection of some molecular outflows, specifically those outflows not oriented along our line of sight (the case of NGC 1068, which has a nuclear molecular outflow detected in multiple transitions, but no outflow signatures in its $\mathrm{OH}$ spectrum, is a clear example; Sec. 4.3).

The $\mathrm{OH}$ absorption studies reveal a broad range of velocities $v_{84}$ from a few 100 $\mathrm{km} \mathrm{s}^{-1}$ up to a few $1000 \mathrm{~km} \mathrm{~s}^{-1}$ (Fig. 21; Veilleux et al. 2013a; Spoon et al. 2013). Interestingly, the outflow velocity does not correlate with the SFR, while it shows a clear correlation with the AGN luminosity. The fastest outflows are systematically seen to be associated with very luminous (quasar-like) AGNs, clearly revealing that luminous AGNs play a primary role in driving fast molecular outflows. In particular, in some cases, the outflow velocity exceeds $1000 \mathrm{~km} \mathrm{~s}^{-1}$. Such fast outflows are difficult to explain with models of starburst-driven outflows. Interestingly, ionized outflows generally have higher velocities, suggesting a different origin of the two phases. However, there are also outflows in which molecular and ionized phases have nearly identical velocities; in these few cases the scenario of molecular gas forming out of cooling from the hot phase may be a viable explanation (see Sect. 2.2.1 and 2.3.2 for more details). We return to this point in Sect. 5.6.
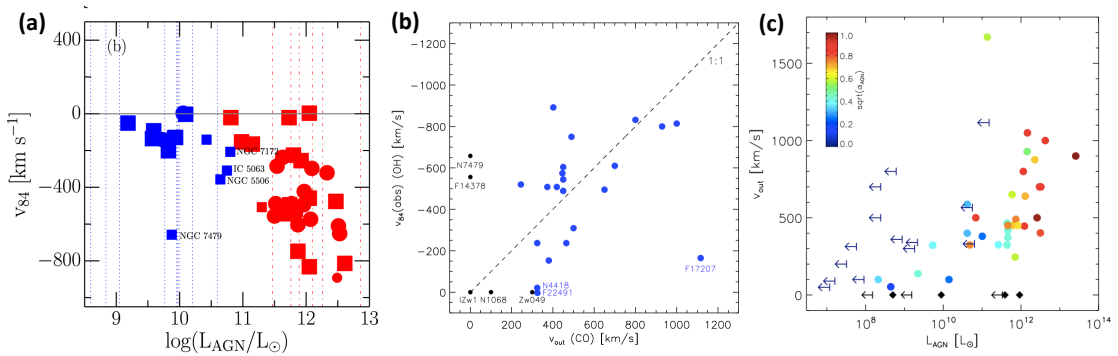

Fig. 21 Velocities of nearby molecular outflows. (a) OH-based 84-percentile outflow velocities in U/LIRGs (red) and BAT AGN (blue), (b) Comparisons of $\mathrm{CO}$ and $\mathrm{OH}$ 84-percentile outflow velocities in nearby gas-rich galaxies. (c) CO outflow velocities as a function of AGN luminosities, color-coded by AGN fraction. Images reproduced with permission from (a) Stone et al. (2016), copyright by AAS; and (b, c) Lutz et al. (2019), copyright by the authors.

In star-forming galaxies, the outflow rate is roughly proportional to the star formation rate (upper right panel of Fig. 22 and Fig. 23a). The molecular outflow rate to 


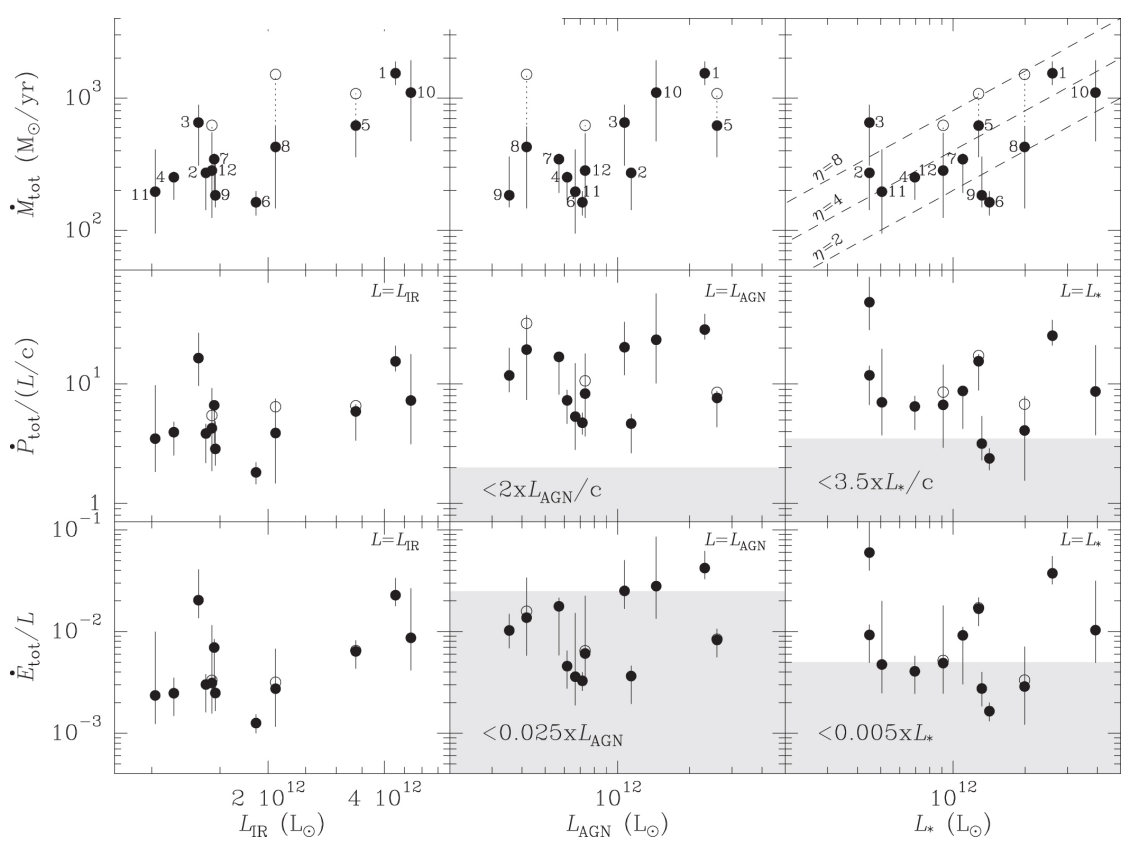

Fig. 22 Energetics of nearby OH-based molecular outflows. The mass outflow rates (top row), momentum rates normalized by the radiation pressure, $L / c$ (middle row), and kinetic energy rates normalized by the luminosity, $L$, are plotted as function of the total infrared luminosity (left column), AGN luminosity (middle), and starburst luminosity (right). Filled circles show the values obtained by ignoring the more uncertain low-velocity components, while open circles include them. Dashed lines in the upper right panel trace different mass-loading factors $\eta \equiv \dot{M} / \dot{M}_{*}=2,4$, and 8 . The grey-shaded rectangles mark the momentum and energy rates that can be supplied by an AGN and a starburst according to various models described in González-Alfonso et al. (2017b). Images reproduced with permission from González-Alfonso et al. (2017b), copyright by AAS.

SFR rate is typically lower than unity. However, when taking into account the contribution from the ionized and atomic neutral phases, the total outflow rate to SFR ratio, i.e. the so-called "outflow mass loading factor", is around unity, as expected and required by models of galaxy evolution in order to regulate star formation (e.g. Finlator and Davé 2008; Davé et al. 2011; Somerville et al. 2015). The same correlation extends to powerful starbursts, although there are indications that it might saturate in the most extreme objects in the early Universe (see Sec. 6.1).

The presence of an AGN can boost the outflow rate, and therefore the mass loading factor, by one or even two orders of magnitude (top-middle panel in Fig.22 and Fig.23a-b), providing further, unambiguous evidence that AGN feedback plays a key role in regulating star formation in galaxies. However, the relation between outflow rate and AGN luminosity has a large scatter. Fluetsch et al. (2019) clarify that part of this scatter is due to the fact that one has to simultaneously take into account the contribution of star formation and AGN when trying to identify scaling relations for outflows, and suggest that a diagram involving a near-linear dependence on both SFR and AGN luminosity greatly decreases the scatter in the outflow scaling relations. The 
scatter decreases even further if one includes also the dependence on the galaxy stellar mass (Fig.23c-d), which is expected to play an additional important role as the deeper gravitational potential well of massive galaxies suppress the development of massive outflows (Eq. (6)). Interestingly, once the dependence on SFR and AGN luminosity are simultaneously taken into account, the dependence on galaxy stellar mass follows the relation $\dot{\mathrm{M}}_{\mathrm{H} 2} \propto \mathrm{M}_{*}^{\alpha}$ with $\alpha=-0.41 \pm 0.25$, i.e. consistent with the theoretical value $\alpha_{t h}=-0.5$ expected by many past models of outflows driven by star formation (Mitra et al. 2015; Somerville et al. 2015; Chisholm et al. 2017). While more recent models and numerical simulations have obtained a more complex dependence on stellar mass (Nelson et al. 2019; Tollet et al. 2019), they are however still broadly consistent with the observations given the current large uncertainties and scatter. Often there is also some level of misunderstanding between theoretical/simulated quantities and observations on what is the definition of "outflow rate", as observationally it is often identified as the rate at which gas leaves the active region of the galaxy, while often in theoretical models the outflow rate refers to the fraction of the gas permanently leaving the galaxy or the halo.

The residual dispersion in these relations is certainly partly due to uncertainties in the measurements, but also likely associated with the flickering nature of AGNs (Sect. 2.1.2). Indeed, black hole accretion can vary on timescales as short as a few years, while the outflow dynamical scales are of the order of at least a few million years (e.g. Schawinski et al. 2015; Gilli et al. 2000). Therefore, AGN-driven outflows can easily outlast the black hole active phase (AGN-quasar) and one would expect a significant population of "fossil" outflows. This phenomenon has been modelled in detail by Zubovas (2018). The observational identification of such "fossil" outflows is not easy, by definition, as the tendency is always to associate the outflow with the currently observed property of the host galaxy. However, recent studies have identified massive and energetic molecular outflows which are extremely difficult to explain in terms of star formation or (currently observed) AGN luminosity (Sakamoto et al. 2014; Fluetsch et al. 2019; Lutz et al. 2019); the most plausible explanation in these cases is that the outflow is the result of a past ejection event produced by a (more active) AGN/quasar phase, that has recently faded.

Various authors have attempted to infer the effect of the observed molecular outflows, especially the AGN-driven ones, on galaxy evolution. Given that molecular gas is the main fuel for star formation, these massive molecular outflows are expected to affect significantly the evolution of star formation in galaxies. In some of the AGN-driven outflows the ejection rate is so high (significantly higher than the star formation rate) that, if maintained, it can potentially clean the galaxy of its molecular gas content within only a few tens million years, hence potentially be effective in totally quenching star formation on short timescales (Sturm et al. 2011; Cicone et al. 2014; Fiore et al. 2017; Gowardhan et al. 2018; Fluetsch et al. 2019). However, these depletion times assume that there is no significant accretion on similar timescales (assumption which may be appropriate locally but not necessarily in the distant universe) and that the observed outflow is not an isolated ejection event but a continuous process (which is not really the case in the blast wave scenario and also in the case of AGN flickering). Moreover, the depletion times are often calculated by using the molecular gas content. If the total gas content is used, i.e. including the atomic H I 
(a)

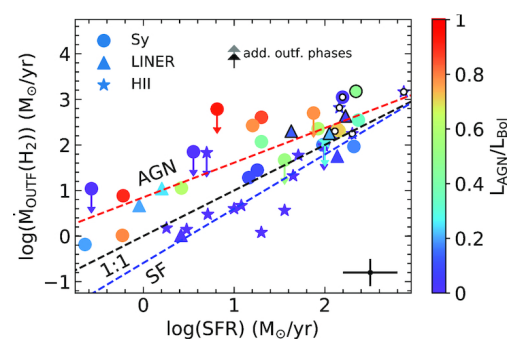

(c)

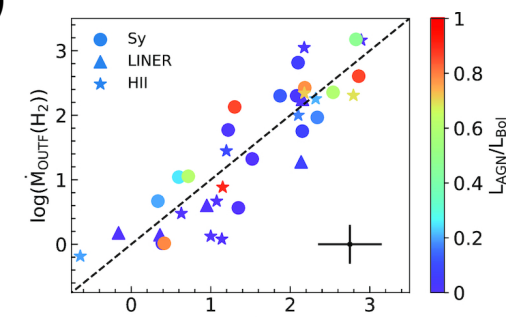

$1.14 \log \left(0.52 \frac{\mathrm{SFR}}{\mathrm{M}_{\odot} / \mathrm{yr}}+0.51 \frac{\mathrm{L}_{\mathrm{AGN}}}{10^{43} \mathrm{erg} / \mathrm{s}}\right)-0.41 \log \left(\frac{\mathrm{M}_{\mathrm{Star}}}{10^{11} \mathrm{M}_{\odot}}\right)$

(e)

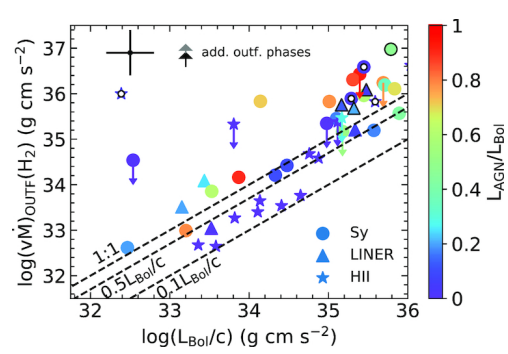

(b)

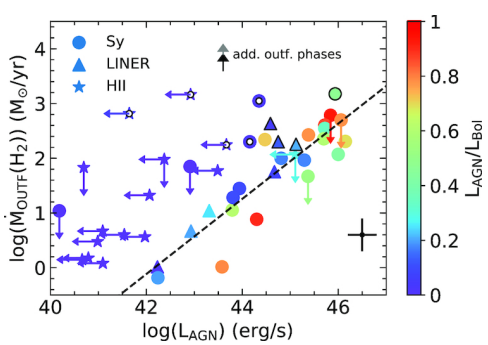

(d)

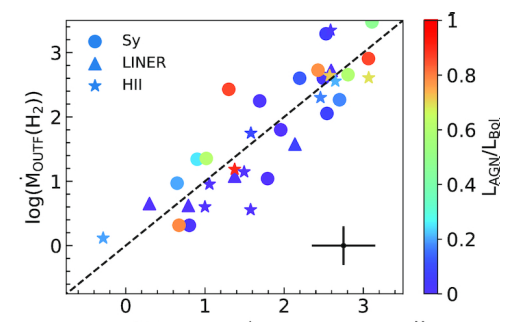

$1.13 \log \left(1.29 \frac{\mathrm{SFR}}{\mathrm{M}_{\mathrm{o}} / \mathrm{yr}}+0.81 \frac{\mathrm{L}_{\mathrm{AGN}}}{10^{43} \mathrm{erg} / \mathrm{s}}\right)-0.37 \log \left(\frac{\mathrm{M}_{\mathrm{Star}}}{10^{11} \mathrm{M}_{\odot}}\right)$

(f)

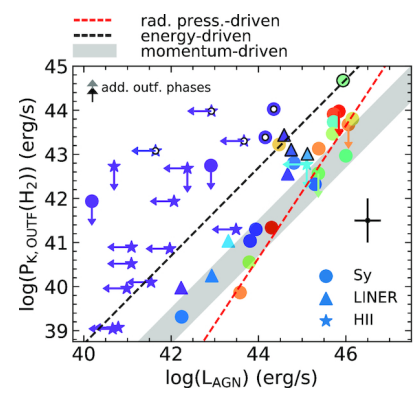

Fig. 23 Energetics of nearby CO-based molecular outflows. (a) Total (ionized + neutral + molecular) mass outflow rates are plotted as a function of SFR. The black dashed line indicates a mass-loading factor of unity, while the red and blue dashed lines are the best fits for the AGN and star-forming galaxies, respectively. (b) Same as (a) but plotted as a function of AGN luminosity. The black dashed line indicates the best fit to the AGN host galaxies. (c) Molecular mass outflow rates as a function of a combination of SFR, AGN luminosity, and stellar mass. The black dashed line represents the best fit to the data. (d) Same as panel (c) but for the total mass outflow rate. (e) Momentum outflow rate as a function of the total radiation pressure, $L / C$. The dashed lines show different ratios of momentum rate to radiation pressure. (f) Kinetic power as a function of the AGN luminosity. The grey band indicates the predictions of momentum-driven outflows, while the black and red dashed lines indicate the predictions for energy-driven and radiation pressure driven outflows. In all panels, the symbols are color-coded according to the AGN fraction, and the shapes are used to differentiate between Seyferts, LINERs, and star-forming galaxies. Images reproduced with permission from Fluetsch et al. (2019), copyright by the authors. 
component, then the resulting depletion timescales are much longer. This suggests that most of these outflows may be effective in cleaning (and quenching) the nuclear region of galaxies, but not globally, across the entire disk. This is indeed expected by detailed, zoom-in 3D numerical simulations, illustrating that even for the most energetic outflows the bulk of the outflow escapes along the path of least resistance (e.g. perpendicular to the galaxy disk in the case of spiral galaxies) leaving the bulk of dense gas in the host galaxy unaffected (Costa et al. 2014, 2015; Gabor and Bournaud 2014; Roos et al. 2015; Hartwig et al. 2018; Richings and Faucher-Giguère 2018b,a; Nelson et al. 2019; Koudmani et al. 2019).

The additional concern about the ejective mode in really being an effective mechanism in quenching galaxies is that generally only a small fraction of the outflowing gas reaches the escape velocity from the galaxy. This will be discussed more extensively in Section 5.6.

Most of the studies reported above refer to wide-angle outflows driven by the thermal or radation pressure from AGN, supernovae or young stellar populations. However, there is growing evidence that radio jets, despite being highly collimated, can also uplift molecular gas and produce wide angle outflows in the ISM of galaxies. In Section 4.5, we discussed in detail four specific cases (Cen A, IC 5063, NGC 1266, and NGC 1377), but evidence is found for other galaxies with radio jets (e.g. Dasyra and Combes 2012; Guillard et al. 2012; Morganti and Oosterloo 2018; Murthy et al. 2019; Fotopoulou et al. 2019; Zovaro et al. 2019; Fernández-Ontiveros et al. 2019). It is generally found that the jet-driven outflowing gas in the molecular phase is as massive as the neutral-atomic phase, or even more massive, and largely exceeding the mass in the ionized phase. The morphology, excitation and general physical properties of jet-driven molecular outflows are consistent with models and numerical simulations in which relativistic jets, while collimated, can generate wide angle molecular outflows by depositing energy into the surrounding medium while piercing through the ISM of the galaxy (Wagner et al. 2012; Mukherjee et al. 2018b,a; Bourne and Sijacki 2017). In the cases of clearly jet-driven outflows the mass loading outflow rates are generally significantly lower than outflows driven by thermal/radiation pressure. However, a systemic study of a large sample of jet-driven outflows is still missing, so it is not really possible to assess the impact of this phenomenon on the evolution of galaxies.

In this context, it is interesting to remind the readers of the recent discovery of molecular outflows that are highly collimated, which also have indication of precession (the best such case, NGC 1377, was discussed in Sect. 4.5; see also Sect. 5.5 below; Sakamoto et al. 2014; Aalto et al. 2016). Such highly collimated outflows may be more common than the few cases identified so far, as their detection requires mapping with angular resolution (and sensitivity) high enough to fully resolve the structure of the molecular outflows, which has become possible only recently. The nature and driving mechanism of these highly collimated molecular outflows is not yet clear. Faint (undetected) or faded radio jets may have been responsible for driving these molecular outflows. Alternatively, these collimated molecular outflows may be driven by a disk-wind similar to those seen in protostars, but scaled up by a large factor. 
Still in connection with radio jets, but on much larger scales, the last few years has seen the ubiquitous detection of molecular filaments in the vicinity of the bright central galaxies (BCG) of galaxy clusters, which are generally radio galaxies. These molecular structure extend from a few kpc to several tens kpc, have molecular gas masses in the range of $10^{8}-10^{10} \mathrm{M}_{\odot}$, and generally are seen to trail the X-ray hot cavity inflated by the radio jets (Russell et al. 2014, 2017b,a, 2019; Tremblay et al. 2018; Vantyghem et al. 2018, 2019; Olivares et al. 2019). The velocity gradients along the filaments are smooth and shallow, and generally inconsistent with freefall. The origin of these large-scale molecular structures is not yet clear. At least in a few cases the mass of the molecular gas in the filament is so large that it is unlikely to result from direct uplifting of molecular clouds by the radio-jet or by the radio-bubbles (e.g. Russell et al. 2017b). Generally, the scenario favoured by most authors is that the molecular filaments result from low-entropy (X-ray emitting) gas uplifted by the radio bubbles and which becomes thermally unstable and therefore cools rapidly forming molecules in situ. As we shall see in the next sections, the gas seems to cool to the point of becoming gravitationally unstable and result in prominent star formation. However, in some cases even the gas cooling from low entropy gas is unable to explain the large masses of gas observed in filaments in the intracluster medium and in these cases sloshing of molecular gas induced by galaxy merging appears to be a reasonable alternative (Vantyghem et al. 2019; Olivares et al. 2019). Finally, it is important to note that in all cases the velocity of these molecular filaments is significantly lower than the escape velocities, implying that this molecular gas will rain back onto the galaxy to fuel additional star formation and black hole accretion, in line with scenarios of chaotic cold accretion and precipitation (e.g. Gaspari et al. 2017)

\subsection{Dust Component}

The evidence for dust in outflows and the halos of galaxies is wide-spread, varied, and quickly growing. Extinction and reddening measurements (Sect. 3.5.1) have a proven track record of successfully detecting dust using background light from the host galaxy itself. Dark dust "worms" and "filaments" seen against the bright optical stellar continuum of nearby edge-on disk galaxies with modest SFRs are the telltale signs that stellar feedback is efficient at transporting dust at least $\sim 2-3 \mathrm{kpc}$ from the sites of star formation (Fig. 8; Howk and Savage 1997, 1999; Thompson et al. 2004). At the other extreme of star formation activity, reddening measurements based on galaxy colors and $\mathrm{H} \alpha / \mathrm{H} \beta$ line ratios have long been known to be a good predictor of deep Na I absorption features and Na I outflows in U/LIRGs (Veilleux et al. 1995; Heckman et al. 2000; Rupke et al. 2005c). More recently, spatially resolved reddening measurements across the host galaxies of $\mathrm{Na}$ I outflows have shown a good spatial correspondence between $E(\mathrm{~B}-\mathrm{V})$ and $N(\mathrm{Na} \mathrm{I})$, proving without a doubt that dust is entrained with the neutral gas in these cool outflows (Rupke and Veilleux 2013b).

In recent years, extinction and reddening of background probes by dusty foreground galaxies has become arguably one of the most powerful techniques to search for dust in the CGM. Following the pioneering work of Zaritsky (1994), studies of the 


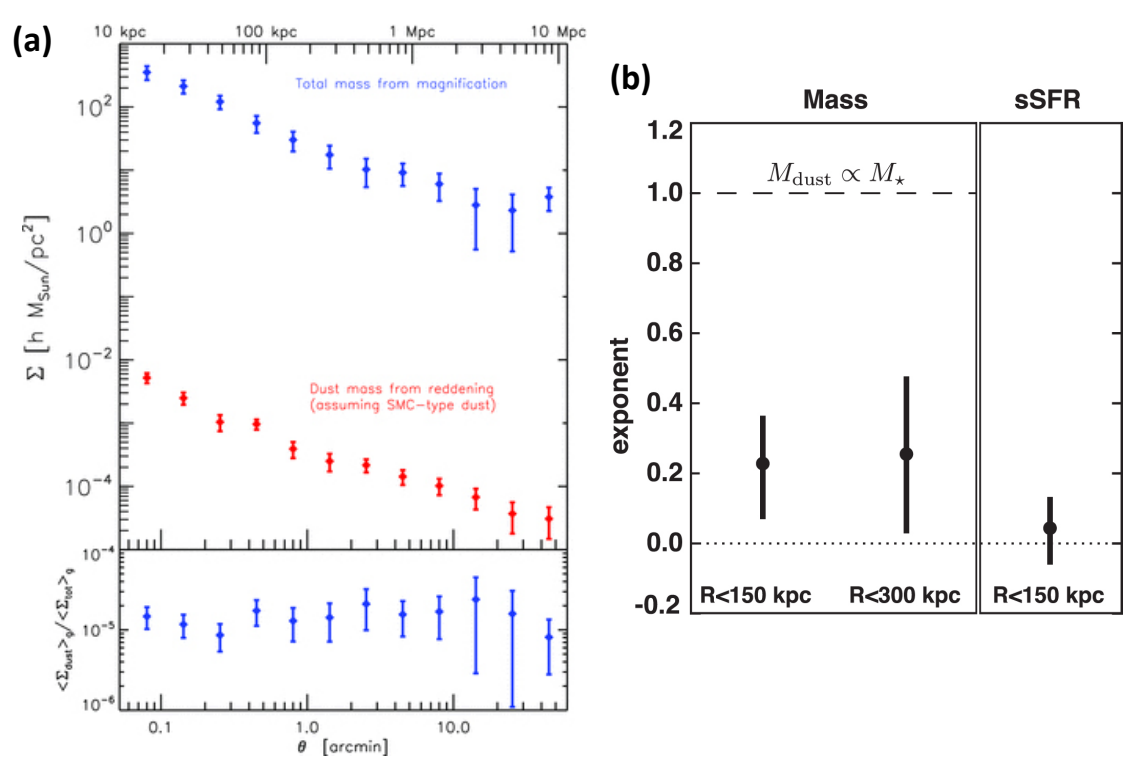

Fig. 24 Circumgalactic and intergalactic dust derived from reddening in nearby galaxies. (a) Mean surface mass density (blue), dust mass density (red), and the ratio of dust to total mass (bottom in blue) as a function of the angular distance (lower scale) and effective projected distance (upper scale) from $z \sim 0.3$ galaxies. (b) Power-law index of the dependence of the circumgalactic reddening on the stellar mass and sSFR in $z \sim 0.05$ galaxies. Images reproduced with permission from (a) Ménard et al. (2010), copyright by the authors; and (b) Peek et al. (2015), copyright by AAS.

reddening measurements of background quasars (Ménard et al. 2010) and passively evolving galaxies ("standard crayons"; Peek et al. 2015) by foreground $z \lesssim 1$ galaxy halos have detected dust from $20 \mathrm{kpc}$ to a few Mpc from galaxies, i.e. extending all the way to the IGM (Fig. 24a). The inferred amount of dust outside of galaxies is comparable to that within galaxies, and the sum of the two adds up to the amount of dust produced in stellar evolution over the entire history of the universe (Fukugita 2011). This remarkable result implies that most of the intergalactic dust survives over a cosmic time $\left(t_{\text {sput }} \simeq 10^{10}\right.$ yrs if $n_{\mathrm{H}} \simeq 10^{-5} \mathrm{~cm}^{-3}$ from Eq. (61)). The circumgalactic reddening shows surprisingly no dependence on sSFR and only a weak correlation with the stellar mass $\left(M_{\text {dust }} \propto M_{*}^{0.2}\right.$; Fig. 24b). The reddening curve derived from these data is SMC-like (steeply rising in the UV) and inconsistent with graphite (no strong $2175 \AA$ bump). The data favor silicate with $a \sim 0.03 \mu \mathrm{m}$ or amorphous carbon with $a \sim 0.01-0.03 \mu \mathrm{m}$, although if the main dust composition in galaxy halos is silicate there is a tension in the grain size between the constraint from the reddening and that from the evolution of extinction with redshifts (Hirashita and Lin 2018). Regardless, these results indicate that large dust grains in the CGM are ruled out, perhaps a sign that the dust grains in the CGM experience erosion as they travel to large distances from the host galaxies. However, these conclusions should be tempered by the fact that some of the circumgalactic dust may be associated with extended gaseous disks rather than cool winds (Smith et al. 2016). A study that takes into account the 
orientation of the sight lines with respect to the minor axis of foreground galaxies should be able to weigh in on the question of the origin of the circumgalactic dust.

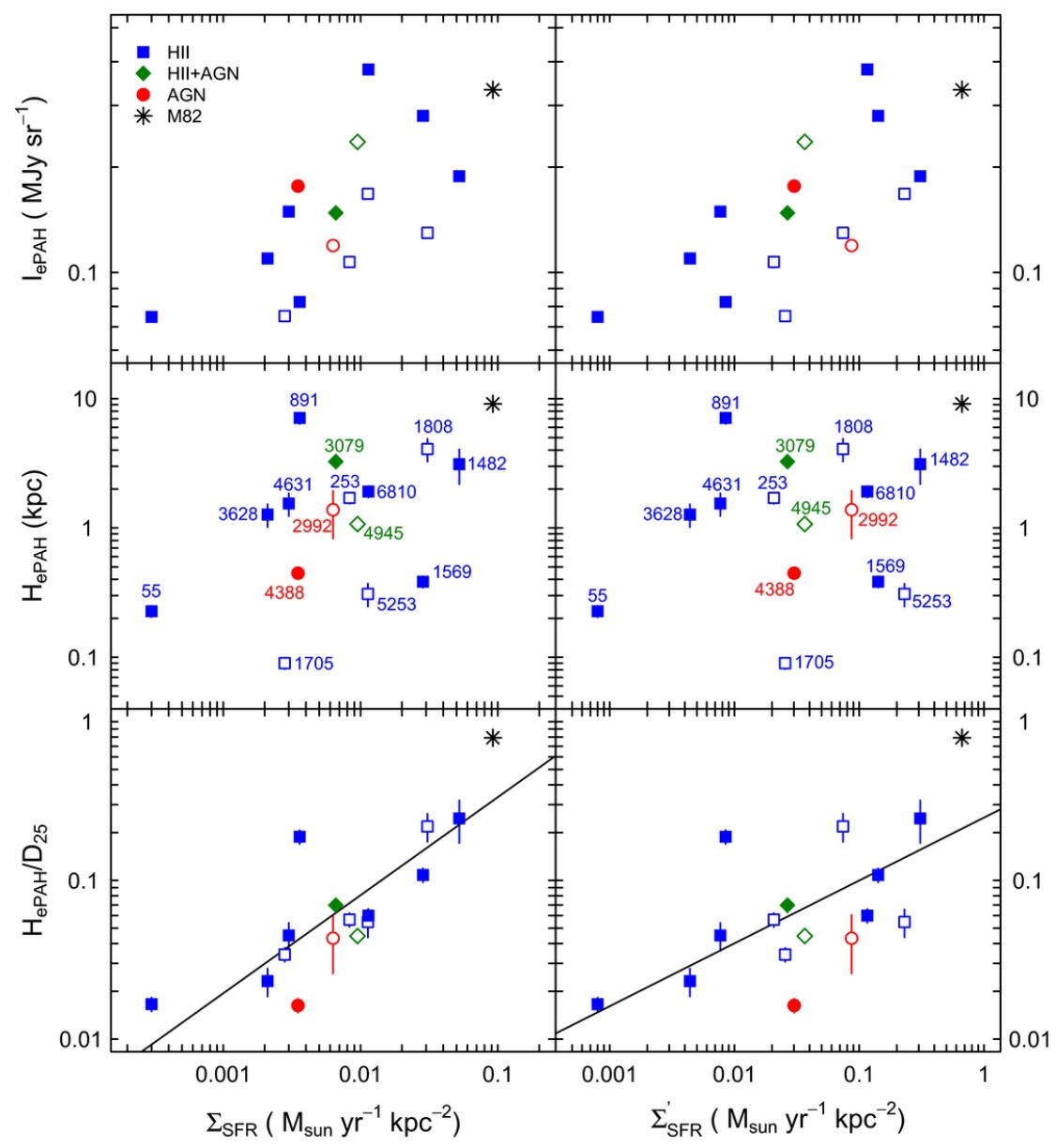

Fig. 25 Extraplanar PAH emission in six nearby disk galaxies. Characteristic extraplanar PAH surface brigthtness (top row), characteristic extraplanar emission height (middle row), and characteristic extraplanar emission height normalized by the stellar disk diameter (bottom row) as a function of the galaxy's SFR surface density (left column) and characteristic SFR surface density (right column). Images reproduced with permission from McCormick et al. (2013), copyright by AAS.

Most of our knowledge on the distribution and properties of the circumgalactic dust in nearby galaxies has come from using direct methods of detection. In the past $\sim 15$ years, ISO, Spitzer and Herschel have been able to directly map the thermal emission from dust in the halos of about two dozen galaxies (Sect. 3.5.3). A linear correlation between the amount of extraplanar PAH emission and SFR has been reported by McCormick et al. (2013). Their results also indicate a correlation between 
the height of the extraplanar PAH emission and the star formation rate surface density, $\Sigma_{\text {SFR }}$ (Fig. 25), although objects with extensive PAH emission (up to $\sim 10 \mathrm{kpc}$ above the mid-plane) and low SFR and $\Sigma_{\text {SFR }}$ do exist (e.g., NGC 5907 and NGC 5529: Irwin and Madden 2006; Irwin et al. 2007, respectively). NGC 4631 is another object which does not fit these simple relations. A rich complex of cool-dust filaments and chimney-like features extends to projected distance of $6 \mathrm{kpc}$ above the plane of this galaxy despite a modest SFR and $\Sigma_{\text {SFR }}$ (Fig. 8d; Meléndez et al. 2015). Star formation in the disk of this galaxy seems energetically insufficient to lift the implied $\sim 10^{8} M_{\odot}$ material out of the disk, unless it was more active in the past or the dust-to-gas ratio in the superbubble region is higher than the assumed Galactic value. Another important factor is the galaxy mass, which sets not only the escape velocity but also the gas pressure in the ISM working against that of the hot bubble created by the $\mathrm{SNe}$ (Sect. 2.2.1). Indeed, the fraction of the dust mass that resides outside of the stellar disks of the few star-forming dwarf galaxies studied so far is higher on average than in larger galaxies, typically $\sim 10-20 \%$ instead of $\lesssim 5 \%$ in the more massive galaxies. This fraction is even higher (perhaps $\gtrsim 50 \%$ ) in NGC 1569 , host of a well-known galactic wind (McCormick et al. 2018, Fig. 26). Evidence for dust grain processing by shocks and other processes has been reported in a few objects. A pixel-by-pixel analysis of the Herschel data on NGC 4631 shows that dust coincident with an 3-kpc X-ray superbubble has a higher temperature and/or an emissivity with a steeper spectral index $(\beta>2)$ than the dust in the disk, possibly the result of the harsher environment in the superbubble. Bocchio et al. (2016) have also found that the abundance of small grains relative to large grains $\sim 2 \mathrm{kpc}$ from the midplane of NGC 891 is almost halved compared to levels in the midplane.

As discussed in Section 3.5.2, galaxy light scattered off of dust ("reflection nebulae") may also be used to detect dust in galaxy halos and constrain its properties (since the scattering cross-section $\sigma_{s, \lambda}$ depends on dust composition and grain size distribution; Sec. 3.5.2). This method has been particularly fruitful in the UV, where dust scattering is efficient and the sky background in space is dark. Systematic searches for vertically extended scattered UV starlight in $\sim 50$ nearby edge-on galaxies with GALEX and Swift have revealed UV halos extending up to $\sim 20 \mathrm{kpc}$ from the galaxy midplanes (e.g., Hodges-Kluck and Bregman 2014; Seon et al. 2014; Hodges-Kluck et al. 2016a; Shinn 2018; Jo et al. 2018). A strong linear correlation between the integrated luminosities of UV halos and the de-reddened luminosities (and thus SFR) of the host galaxies is observed, fitting both starburst and less actively star-forming galaxies. However, starburst galaxies with known ionized galactic winds have qualitatively different halos that are more extensive and have complex filamentary structures that often match $\mathrm{H} \alpha$ and cool-dust infrared filaments (Fig. 27; Hodges-Kluck et al. 2016a; Jo et al. 2018). Dust masses of a few $\times 10^{6} M_{\odot}$ (or gas masses of a few $10^{8}$ $M_{\odot}$ for a MW-like dust-to-gas ratio) within $2-10 \mathrm{kpc}$ of the disk are implied, based on Monte Carlo radiative transfer (MCRT) scattering models and relatively simple assumptions about the geometry of both the scattering halos and galaxy disk luminosity distribution (Hodges-Kluck et al. 2016a; Baes and Viaene 2016). A good match is found between the dust masses derived from the UV data and those derived from FIR emission for the few objects where this comparison is possible (Hodges-Kluck et al. 2016a). 


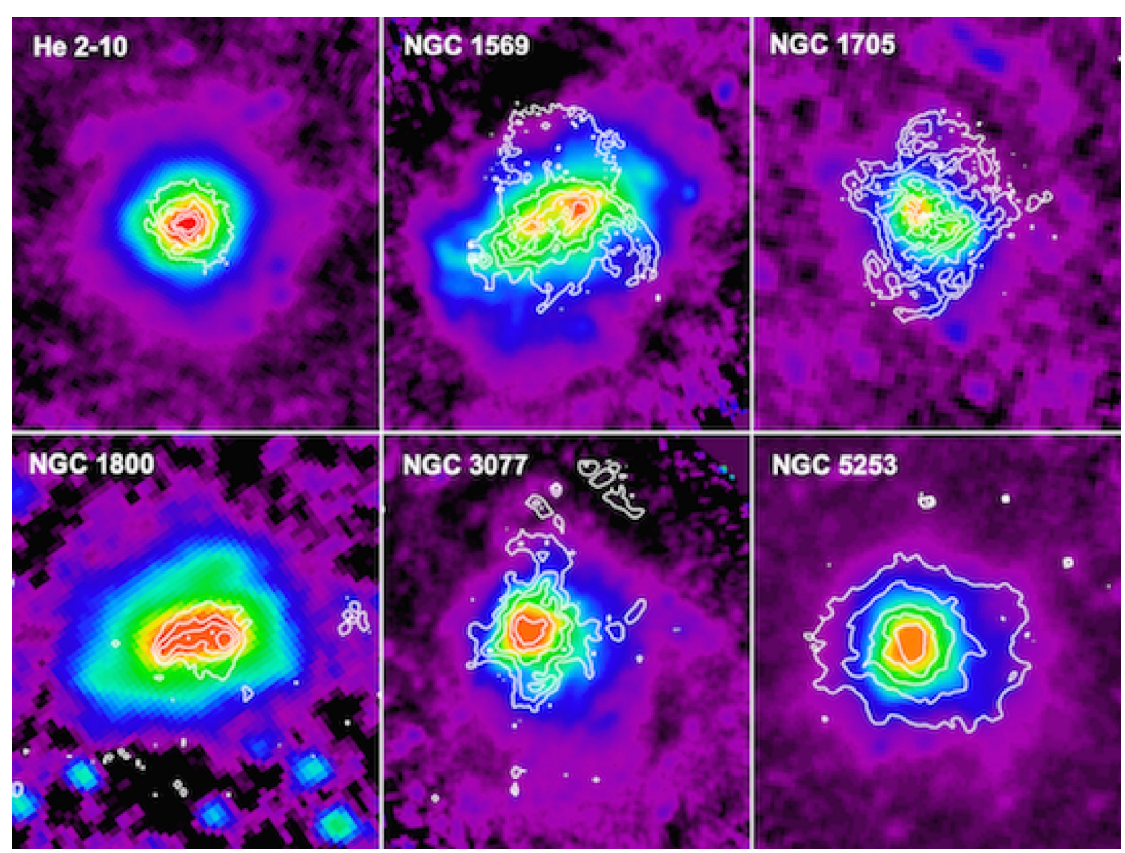

Fig. 26 Circumgalactic infrared dust emission in nearby dwarf galaxies. Herschel PACS $160 \mu \mathrm{m}$ maps are overlaid with $\mathrm{H} \alpha$ contours to compare the distribution of the cold dust with that of the warm ionized material. Images reproduced with permission from McCormick et al. (2018), copyright by the authors.

In recent years, differential elemental depletion onto dust grains (Sect. 3.5.5) has independently confirmed the existence of dust in the halos of $z \lesssim 1$ galaxies inferred from reddening, infrared, and UV measurements. But, for all practical purposes, this method has so far been limited to the stronger metal-line absorbers with $N_{\mathrm{H}} \gtrsim 10^{18}$ $\mathrm{cm}^{-2}$, where the UV absorption lines from ions of key volatile and refractory elements with different depletion factors (e.g., $\mathrm{Zn}, \mathrm{O}, \mathrm{P}, \mathrm{S}, \mathrm{Si}, \mathrm{Mg}, \mathrm{Mn}, \mathrm{Cr}$, and Fe) can be measured to derive the total column density of each element, and then compared with the hydrogen column density measured from the strength of the damping wings of Ly $\alpha$ or the H I 21-cm emission line along the same line of sight (see Sect. 3.5.5 for possible caveats). Dust inferred using this method has been reported in the Milky Way Fermi Bubbles (Savage et al. 2017), the galactic fountain of NGC 891 (Bregman et al. 2013; Qu et al. 2019), some DLAs at $z \lesssim 1$ (Péroux et al. 2006; Rahmani et al. 2016), and a few $z \lesssim 1.5$ LLS with $N_{\mathrm{H}} \simeq 10^{19}-10^{20} \mathrm{~cm}^{-2}$ ("sub-DLAs"; Meiring et al. 2009a,b), although not all of them (e.g., Quiret et al. 2016). On the other hand, dust depletion in $z \lesssim 1$ LLS with $N_{\mathrm{H}}<10^{19} \mathrm{~cm}^{-2}$ has been found to be modest, largely consistent with dust-free gas (e.g., Lehner et al. 2013, 2019). Most of these LLS are metal-poor, mostly ionized, and not believed to be directly associated with cool winds.

The results presented so far have largely focused on star-forming systems. However, it is clear that dust is also associated with AGN-driven outflows on all scales. 


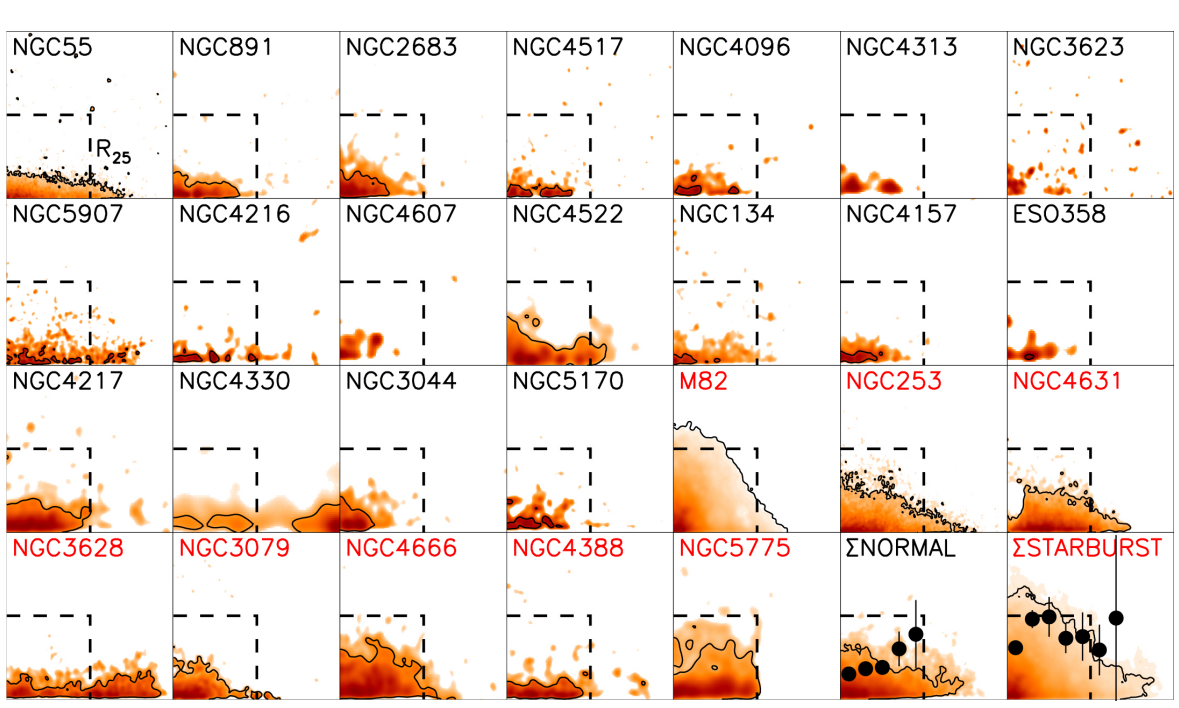

Fig. 27 Dust scattering in nearby edge-on star-forming disk galaxies. Combined UV image stacked in the first quadrant are shown relative to the optical radius, $R_{25}$ (indicated by the dashed lines). Starburst galaxies are labeled with red names. The final two panels show the combined normal and starburst galaxies, respectively, with scale heights measured as a function of galactocentric radius shown as points. Image reproduced with permission from (Hodges-Kluck et al. 2016a), copyright by AAS.

We have already discussed the case for dust shielding to explain the AGN-driven $\mathrm{Na}$ I outflows detected on small scales in FeLoBALs (e.g., Mrk 231; Sec. 4.4) and on kpc scales in several AGN-dominated ULIRGs (sec. 5.1). The location of the dust in LoBALs in general is uncertain, which makes the location and energetics of the outflows equally uncertain (Eq. (40); Dunn et al. 2010). In a few low-z cases, the same dust that is responsible for the anomalously red continuum colors of LoBALs and FeLoBALS seems to affect the kpc-scale narrow-line emission in these objects so it must be located outside of the outflow region (Dunn et al. 2015). This is not to say that none of the dust takes part in the FeLoBAL outflows, but this dust may have only subtle effects on the overall spectrum of these objects (e.g., UV extinction without significant reddening; Veilleux et al. 2016; Hamann et al. 2017).

For completeness, we should mention that evidence for dust in the ionized winds of AGN outside of the dust sublimation radius is also quickly growing. Mid-infrared interferometry of nearby Seyfert galaxies with VLT/MIDI has revealed that most of the $12 \mu \mathrm{m}$ emission on scale of tens to hundreds of parsecs originates from optically thin dust in the polar region of the inner torus, likely entrained in a ionized wind (Hönig et al. 2012, 2013; Burtscher et al. 2013; Tristram et al. 2014; López-Gonzaga et al. 2014, 2016; Asmus et al. 2011, 2016; Hönig and Kishimoto 2017; Stalevski et al. 2019; Leftley et al. 2019). Recently, Mehdipour and Costantini (2018) reported the presence of dust in the X-ray wind of the nearby Seyfert galaxy IC 4329A, based on reddening measurements and the strengths of X-ray edge features from $\mathrm{O}, \mathrm{Si}$, and $\mathrm{Fe}$ that indicate dust depletion. Baron and Netzer (2019a,b) have also recently discovered a direct connection between ionized [O III] outflows and the presence of 
dust emission at 10-30 $\mu \mathrm{m}$ in the SED of SDSS AGN. Using Eq. (62), the measured dust temperature $T_{\text {dust }}=100-200 \mathrm{~K}$ is used to derive the location of this dust, $r \sim 500 \mathrm{pc}$, hence the size and energetics of the [O III] outflows in AGN. Some of these dusty AGN-driven outflows may carry dust to CGM scales and help explain the large $(r \gtrsim 5-10 \mathrm{kpc})$ scattering cones detected at $\sim 3000 \AA$ and in polarized light around obscured quasars (Zakamska et al. 2005; Obied et al. 2016). These cones have opening angles and directions that match the blueshifted side of the [O III] outflows. The scattering medium is inferred to be dusty and photoionized by the quasar.

\subsection{Driving Mechanisms in AGN-dominated Systems}

The most powerful, most massive, and fastest cool outflows are associated with quasars. As discussed in Section 2.2.1, the most effective driving mechanism is the so-called "blast-wave" energy-driven mode. In this scenario the very fast $(v \sim 0.01-0.1 \mathrm{c})$ and energetic nuclear wind generated by the AGN radiation pressure produces a blast wave that expands with little radiative losses, i.e. energy-conserving (e.g. King 2010; Zubovas and King 2012; King and Pounds 2015; Faucher-Giguère and Quataert 2012). In this model the cold molecular/atomic phase is accelerated either as a result of the galaxy ISM being entrained/shocked in/by the hot, fast outflow, or as direct cooling and fragmentation of the hot phase (see Sec. 2.3). In this scenario the kinetic power of the outflowing gas is expected to be of the order of a few percent of the AGN radiative luminosity (typically $\dot{\mathrm{E}}_{\text {out }} \approx 0.05 \mathrm{~L}_{\mathrm{AGN}}$ ). Moreover, as mentioned in Section 2.2.1, the energy conserving nature of the outflow implies that the outflow undergoes a momentum boost, with respect to the momentum of the driving radiation radiation pressure, by a factor of $f v_{\text {wind }} / v_{\text {out }}$ from eq. (8), i.e. typically a few tens unless the efficiency $f$ to transform the kinetic energy of the nuclear wind into bulk motion of the large-scale outflow is $<<1$ (most popular models expect $\dot{p}_{\text {out }} \approx 10-20 L_{A G N} / c$ ).

Various studies have attempted to test the blast-wave scenario by constraining the kinetic power and momentum rate in AGN-driven outflows (Cicone et al. 2014; Tombesi et al. 2015; Nardini et al. 2015; Feruglio et al. 2015, 2017; Veilleux et al. 2017; Rupke et al. 2017; Fluetsch et al. 2019; Bischetti et al. 2019; Lutz et al. 2019). Large uncertainties in both quantities make a clear, solid assessment difficult to achieve. Moreover, in several galaxies the outflow is also partly, or mostly, driven by star formation, which complicates the interpretation. Despite all these caveats, the emerging picture is that AGN-driven outflows span a broad range in terms of $\dot{\mathrm{E}}_{\text {out }} / \mathrm{L}_{\mathrm{AGN}}$ and in terms $\dot{\mathrm{p}}_{\text {out }} /\left(\mathrm{L}_{\mathrm{AGN}} / \mathrm{c}\right)$. However, most of the objects seem to lie below the prediction expected by the blast-wave, energy-conserving scenario. Figures 22 and 23 show recent compilations of momentum and energy rates in galaxies in which the molecular outflow is mostly/partly AGN-driven. While a few galaxies are consistent with the expectation of the blast-wave scenario, the majority of them are below the blast-wave scenario expectations, some by orders of magnitude, which is difficult to reconcile in terms of erroneous assumption on the outflow geometry or even by assuming that a significant fraction of the outflow is in the ionized phase (for a significant fraction of these systems however the ionized phase is measured 
and found not to contribute significantly or, anyhow, not by the large factor needed to reconcile observations with the blast-wave scenario).

For a limited number of galaxies with measured molecular or atomic cold outflows, the momentum rate of the nuclear driving wind is also known through $\mathrm{X}$ ray observations (Fig. 28). In these few cases, the momentum boost can be obtained through a direct comparison between the momentum rate of the large-scale outflow and the nuclear wind. The first and still the best case of a fast quasar accretiondisk wind in a system with a massive molecular outflow was reported in the ULIRG F11119+3257 by Tombesi et al. (2015) and confirmed by Tombesi et al. (2017). As mentioned in Section 4.4, in Mrk 231 the momentum rate of the large-scale cold outflow appears consistent with the nuclear wind boosted by $v_{\text {wind }} / v_{\text {out }}$ (Feruglio et al. 2015), i.e. with the (blast-wave) energy conserving scenario. A few additional sources seem consistent with this scenario (Nardini et al. 2015). However in other cases the momentum rate of the large scale outflow is significantly lower than the expectations from the energy conserving scenario and nearly consistent with the momentumconserving scenario (Fig. 28; Veilleux et al. 2017; Feruglio et al. 2017; Bischetti et al. 2019; Smith et al. 2019).

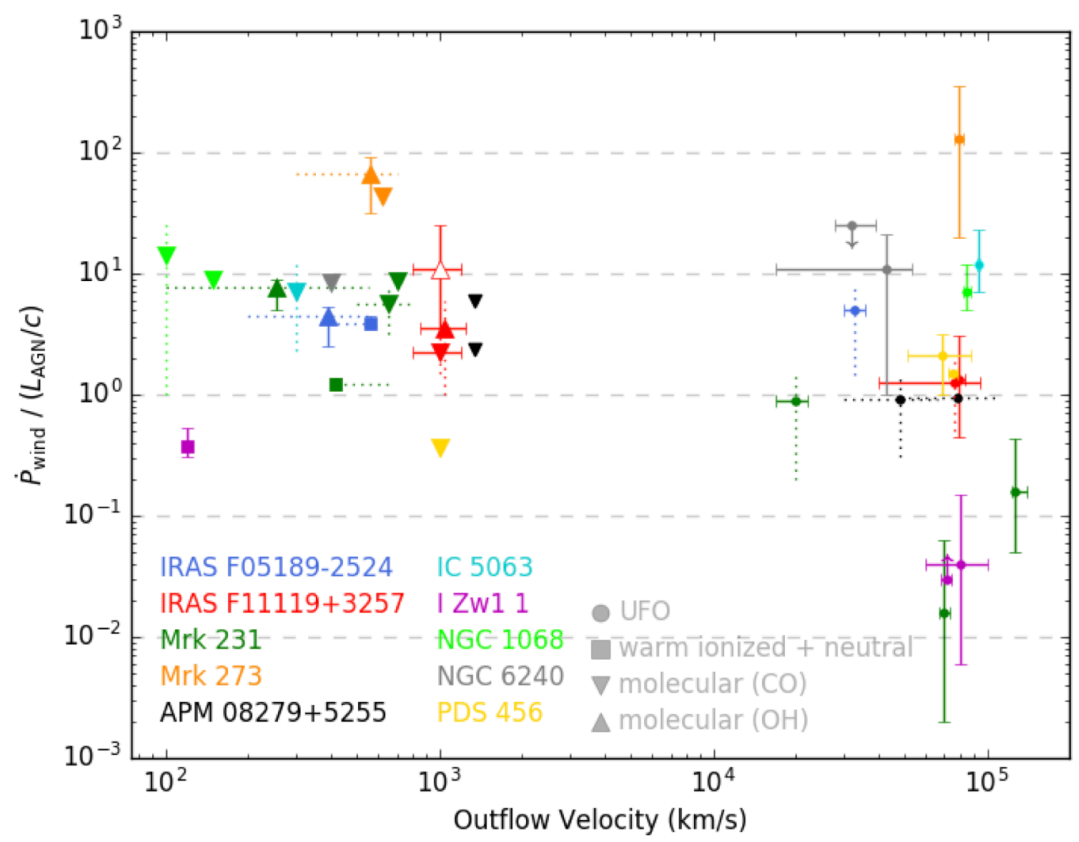

Fig. 28 Driving mechanisms of cool outflows in nearby AGN-dominated systems. Compilation of the momentum outflow rates normalized by the AGN radiation pressure for objects with both large-scale cool outflows (left) and nuclear X-ray winds ("UFO = ultra-fast outflow"; right). The color of each symbol identifies the object and the shape of the symbol indicates the method used to derive the plotted quantities. The open triangle of F11119+3257 is the instantaneous momentum rate from Tombesi et al. (2015); all other data points are time-averaged momentum rates. Images reproduced with permission from Smith et al. (2019), copyright by AAS. 
One likely explanation for these results is that, although energy-driven, the nuclear AGN-powered wind does not couple efficiently with the galaxy ISM (i.e. small values of $f$ in eq. (8)) and that most of the energy escapes along paths of least resistance (e.g. perpendicular to the galaxy disk in the case of regular late-type galaxies) without affecting the bulk of the ISM in the galaxy (especially the dense, star-forming component of the molecular gas). This (not really unexpected) result is obtained by most of the detailed numerical hydrodynamical simulations (Costa et al. 2014; Gabor and Bournaud 2014; Roos et al. 2015; Costa et al. 2015, 2018b; Hartwig et al. 2018; Henden et al. 2018; Koudmani et al. 2019; Nelson et al. 2019). Note that these scenarios do not imply that the AGN-driven wind does not have any major effect on the star formation in the host galaxy. Indeed, the energy injected into the galaxy halo results into heating of the CGM, which reduces or can even suppress cold accretion onto the galaxy. In the latter case star formation in the galaxy would quench as a consequence of 'starvation', i.e. lack of fresh gas delivered to the galaxy to fuel further star formation. This is essentially the so-called 'delayed' or 'preventive' feedback mode, in which the energy injected into the halo does not happen through radio-jets but through wide-scale outflows. Indirect observational evidence of this mode being responsible for the quenching of star formation in galaxies has been suggested by various studies (e.g. Woo et al. 2017; Peng et al. 2015; Trussler et al. 2018).

An alternative scenario to explain the observed trends is that these AGN-driven outflows are primarily caused by direct radiation pressure onto the dusty clouds of the galaxy (Fabian 2012; Thompson et al. 2015; Ishibashi and Fabian 2017; Ishibashi et al. 2017; Costa et al. 2018a; Ishibashi and Fabian 2018; Ishibashi et al. 2018b). This scenario implies lower outflow momentum rates and lower energetics, and can potentially account for the scatter and lower values of these quantities with respect to the energy-driven predictions. As discussed in Section 2.2.2, this scenario was initially deemed implausible because of the Kelvin-Helmholtz and Rayleigh-Taylor instabilities that are expected to develop in this scenario and which would disrupt the outflowing clouds (Scannapieco and Brüggen 2015; Brüggen and Scannapieco 2016; Ferrara et al. 2016). However, more recent works have shown that if the clouds are dense enough (as observed in many outflows now) their core can survive (Decataldo et al. 2019) and additional cold gas can also potentially cool in the tail of the outflowing clouds (Fig. 4d; Gronke et al. 2018; Gronke and Oh 2019). However, even if the gas clouds survive radiation (or ram) pressure acceleration, they have difficulties accounting for outflows that have large momentum rates and high kinetic powers. Multiple scattering of IR photons in a very dense, optically thick medium can potentially boost the momentum rate up to $2-5 \mathrm{~L}_{\mathrm{AGN}} / \mathrm{c}$ (Thompson et al. 2015; Ishibashi and Fabian 2015, 2018; Costa et al. 2018a). Recent versions of these models predict a super-linear relation between outflow rate and $\mathrm{L}_{\mathrm{AGN}}$, which seems supported by recent observations (Fluetsch et al. 2019). However, these models still struggle to produce the large kinetic powers observed in some of the most powerful quasardriven outflows (in these models typically $\dot{\mathrm{E}}_{\text {out }}<0.01 \mathrm{~L}_{\mathrm{AGN}}$ ).

As discussed in Sect. 4.5 and 5.2 (see also Sect. 5.5 below), ram pressure from radio jets also contributes to driving cool outflows in some objects. However, the lack of a correlation between outflow rate and radio loudness in AGN with known molecular outflows suggest that radio jets are not a primary mechanism for most of 
the molecular outflows (Fluetsch et al. 2019). This does not mean that when a radio jet is present in a galaxy it is not the primary driver of an outflow. The few detailed studies carried out so far indicate that, when a radio jet is present, there are no clear galaxy or AGN properties defining whether the molecular outflow is primarily driven by thermal pressure, or radiation pressure, or the radio jet (or even by SNe associated with star formation); each individual case has its own specific peculiarities.

\subsection{Compact Obscured Nuclei (CONs): A Special Evolutionary Phase?}

Herschel-based studies of local molecular outflows (Sect. 5.2; Veilleux et al. 2013a) have shown that $\mathrm{OH} 119 \mu \mathrm{m}$ outflows are ubiquitous among dusty U/LIRGs with deep $\mathrm{OH}$ and $9.7 \mu \mathrm{m}$ silicate-dust absorption features (Sect. 3.5.1). This is consistent with a scenario where molecular outflows with an average opening angle of $\sim 145^{\circ}$ are present in essentially all obscured systems, but subside once the AGN clears a path through the obscuring material. González-Alfonso et al. (2017b) have found that the most deeply buried sources (based on the depth of $\mathrm{OH} 65 \mu \mathrm{m}$ ) split into two subsets (groups I and III in their Fig. 4), where group III galaxies do not show fast $\mathrm{OH} 119$ outflows, despite having the same $W_{\mathrm{eq}}(\mathrm{OH}$ 65) as the galaxies in group I. Falstad et al. (2019) have recently shown that the most obscured galaxy nuclei, the CONs (Sect. 3.4.6), in fact constitute the group with high $W_{\text {eq }}(\mathrm{OH} 65)$, but without OH 119 outflows (Fig. 29a). The CONs are more obscured than the galaxies with fast $\mathrm{OH} 119$ outflows, showing that $W_{\text {eq }}(\mathrm{OH}$ 65) alone cannot identify the most deeply obscured sources. Instead, their identification requires using highly excited lines at longer, mm wavelengths (such as vibrationally excited HCN; Sect. 3.4.6). There is evidence for inflows of molecular and atomic gas into the central regions of some CONs (e.g., González-Alfonso et al. 2012; Costagliola et al. 2013; Sakamoto et al. 2013; Aalto et al. 2015b; Falstad et al. 2015; Aalto et al. 2019). It has therefore been suggested that CONs represent a brief evolutionary phase before mechanical feedback from the obscured starburst or AGN becomes important and creates a detectable outflow. Another possible explanation of these results is that the morphology (collimation), size, and orientation of the molecular outflows in CONs are different from those in galaxies of less extreme obscuration (Falstad et al. 2019). Since the CONs are extremely obscured, it is also possible that their outflows are obscured even in the far-infrared. To understand the difference in outflow structure with varying degree of obscuration (and nature and strength of the buried activity) requires multi-wavelength observations at high spatial resolution.

Recent mm-wave studies of the CON ULIRG Arp 220 have revealed the presence of a fast $\left(840 \mathrm{~km} \mathrm{~s}^{-1}\right)$ collimated molecular and dusty outflow in its western nucleus (Fig. 29b; Sakamoto et al. 2017; Barcos-Muñoz et al. 2018). Interestingly, the collimated molecular outflow was found in $\mathrm{HCN}$, not (as usual) in $\mathrm{CO}$. The outflow is $120 \mathrm{pc}$ long with a dynamical time scale of $10 \mathrm{Myr}$ and a mass outflow rate $>70 M_{\odot} \mathrm{yr}^{-1}$. It is oriented almost perpendicular to the line-of-sight and also shows opaque dust emission at its base. It is not clear if the driving source is primarily a compact starburst or a dusty AGN. A slower outflow has been detected before in absorption (e.g. in $\mathrm{HCO}^{+}$and $\mathrm{SiO}$; Sakamoto et al. 2008; Tunnard et al. 2015), and a 

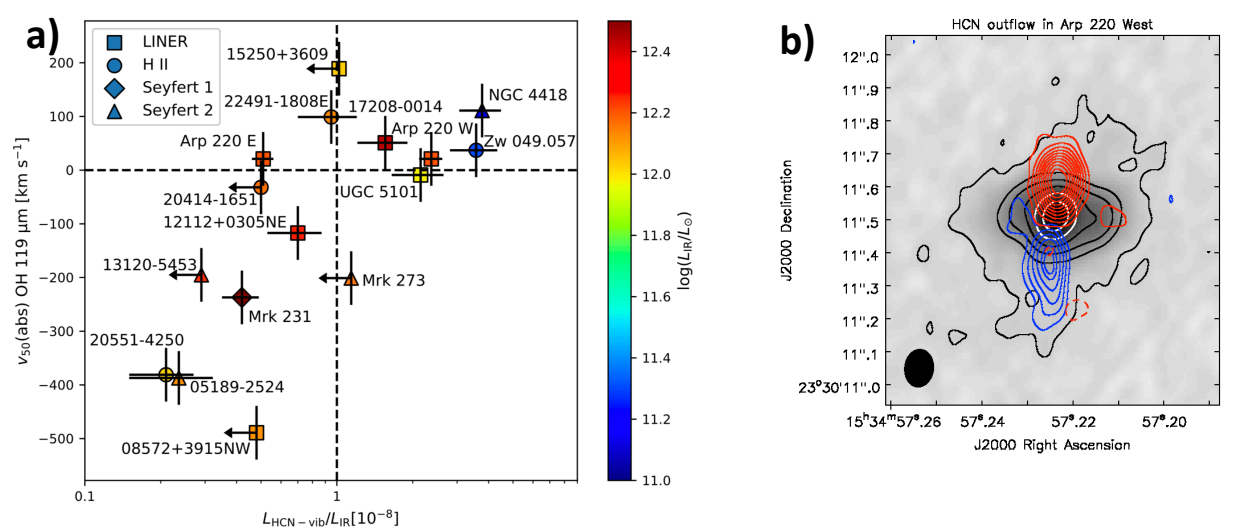

c)

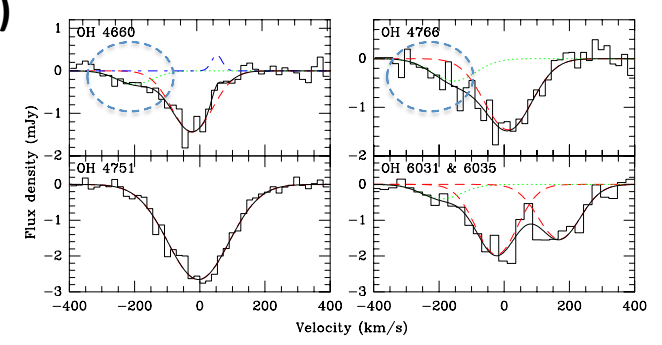

d)

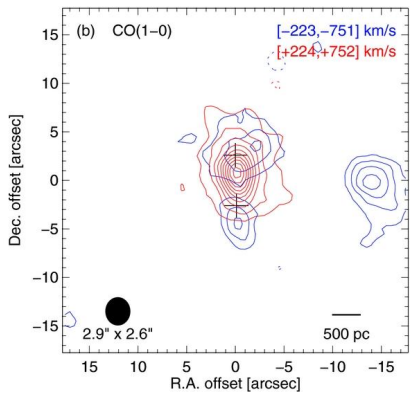

Fig. 29 Compact Obscured Nuclei (CONs). (a) Median far-IR OH absorption velocity as a function of the mm-wavelength $\mathrm{HCN}$-vib luminosity relative to the total infrared luminosity. Squares, circles, diamonds, and triangles represent LINER, HII, Seyfert 1, and Seyfert 2 optical spectral types, respectively. Colors indicate the total infrared luminosity of each system. (b) Greyscale (+black and white contours) 92 $\mathrm{GHz}$ continuum image of the western nucleus of Arp 220. The red and blue contours show the red- and blueshifted HCN1-0 high-velocity emission. (c) VLA OH absorption lines at $\mathrm{cm}$ wavelengths revealing a fast outflow in the LIRG Zw049.057. (d) Collimated molecular outflow emerging from the southern nucleus of the LIRG merger NGC 3256. This nucleus is likely not a CON, but a dormant AGN where the outflow serves as a relic indicator of its past activity. Images reproduced with permission from (a) Falstad et al. (2019) and (c) Falstad et al. (2017), copyright by ESO; and (b) Barcos-Muñoz et al. (2018) and (d) Sakamoto et al. (2014), copyright by AAS.

faint, fast $\left(-700 \mathrm{~km} \mathrm{~s}^{-1}\right) 119 \mu \mathrm{m} \mathrm{OH}$ absorption wing may also be present in this object (Veilleux et al. 2013a), but the connection between the collimated outflow and these various blueshifted absorption structures will require further investigation. Another intriguing case is the CON ULIRG IRAS 17208-0014, where the OH $119 \mu \mathrm{m}$ absorption profile does not show any extended blue wing, but a fast $\left(750 \mathrm{~km} \mathrm{~s}^{-1}\right)$ CO outflow is detected (García-Burillo et al. 2015). It appears collimated but its morphology is not yet fully mapped and hence the origin of the discrepancy between the $\mathrm{OH}$ and $\mathrm{CO}$ results is not clear. The mass, energy, and momentum budgets of the molecular outflow in IRAS 17208-0014 seem to require the existence of a hidden AGN. Other very obscured galaxies and CONs with outflows revealed at longer wavelengths include Zw049.057, ESO 320-G030, IC 860 and NGC 4418 (Baan et al. 
1989; Pereira-Santaella et al. 2016; Falstad et al. 2017; Fluetsch et al. 2019; Aalto et al. 2019). NGC 4418 and IC 860 have wide-angle, large-scale v-shaped dust lanes along the minor axis, and NGC 4418 shows a slow hot minor-axis flow similar to the galactic wind in M82 (Ohyama et al. 2019). The link, if any, between the molecular outflows and these optical features is not yet understood. In the case of NGC 4418, the galactic wind may be powered by an extended 10 Myr old starburst.

In summary, several of the CONs show fast molecular outflows revealed by highresolution mm-wave observations of $\mathrm{CO}$ and/or HCN. In some cases, their morphologies indicate highly collimated outflows, without obvious radio counterparts. The CON outflows may also be physically smaller than those seen in less obscured dusty galaxies, consistent with an evolutionary sequence. Note, however, that collimated, apparently radio-quiet, outflows are not restricted to CONs; they are also found in objects of less extreme obscuration. One example is the collimated molecular outflow associated with the southern nucleus of the merger NGC 3256 (see Fig. 29d; Sakamoto et al. 2006, 2014). It is a fast $\left(\sim 2000 \mathrm{~km} \mathrm{~s}^{-1}\right)$ outflow likely powered by a (now dormant) AGN.

\subsection{Fate of the Outflowing Material}

Three questions arise when discussing the fate of the cool outflowing material: (1) does it escape the host galaxy altogether? (2) Does it experience a phase transition between cold molecular, cool neutral-atomic, and warm/hot ionized? (3) Does it collapse to form stars? The data at $z \lesssim 1$ provide the best constraints on these three important questions.

The first of these questions has been addressed in several studies by comparing the measured radial velocity of the cool outflowing gas with the minimum requirement that it should exceed the escape velocity, often assuming $v_{\mathrm{esc}} \simeq(2-3) v_{\text {circ }}$, where the lower multiplicative factor is for CGM-scale gas while the higher multiplicative factor is for kpc-scale material (see Sec. 2.4). The fraction of neutral-atomic and molecular outflowing material that meets this simple requirement generally is less than $20 \%$, often consistent with zero (e.g., Heckman et al. 2000; Rupke et al. 2005c; Martin 2005; Rubin et al. 2014; Schroetter et al. 2015, 2016, 2019; Fluetsch et al. 2019; Bouché et al. 2012). The few cases where the cool material has velocities that exceed the escape velocity often are those where the velocities are measured down-the-barrel and on small scales ( $r \lesssim 1 \mathrm{kpc}$; e.g., Tremonti et al. 2007; Weiner et al. 2009; Veilleux et al. 2013a; Chisholm et al. 2017; González-Alfonso et al. 2017b). This perhaps indicates that drag forces are subsequently important in slowing down the material as it travels to the halo. However, one should also keep in mind the strong observational bias against detecting escaping outflows on CGM scales since (1) the volume and column densities, covering factor, and surface brightness of the outflowing clouds likely decrease with increasing radius (e.g., Krumholz et al. 2017b), and (2) the escaping clouds at large radii will have only modest velocities, of order $\sqrt{2} v_{\text {circ }}$, for ballistic motions. If instead the material accelerates for some time as it moves away from the center of the galaxy, as in the cases of thermally and radiatively-driven winds (Sect. 2.2.1 and 2.2.2), then the material at small distances from the galaxy with ve- 
locities below escape speed may nonetheless be able to escape because it is still being accelerated. On CGM scales, this accelerated gas may be faster than the ballistically launched material but will remain hard to detect for the reasons listed earlier under (1).

While the current kinematic data, taken at face value, suggest that the cool material generally does not escape the galaxy, this does not necessarily mean that none of the outflowing material makes it out of the galaxy and into the IGM. Indeed, one very real possibility is that the cool outflowing material becomes ionized as it travels to the halo, so it is also important to look at the kinematics of the other warmer gas phases in the outflows. The high wind velocity implied by the measured X-ray temperature of the entrained gas in galactic winds $\left(v_{\mathrm{X}} \simeq 500-900 \mathrm{~km} \mathrm{~s}^{-1}\right)$ has long been used as an argument that a significant fraction of the hot ionized gas may be escaping from dwarf galaxies (Martin 1999), including perhaps M 82 (Lehnert et al. 1999). The measured velocities of the warm ionized phase (traced by emission and absorption lines of e.g. $\mathrm{H} \alpha$, [O III] 5007, Si IV, C IV, and O VI) are generally smaller than $v_{\mathrm{X}}$, but they often - though not always - exceed the velocities of the cooler material (Veilleux et al. 2013a; Rupke et al. 2017; Rodríguez del Pino et al. 2019). The fraction of ionized gas that escapes the host galaxy is therefore higher than that of the cool gas, although it is still modest. Moreover, one should take into account that semi-ballistic motions are not the only way for the gas to escape the halo. Indeed, recent models and simulations have shown that under certain conditions the main effect of the AGN- and $\mathrm{SN}$-driven outflows is to create hot bubbles that become buoyant within the galaxy halo, hence the gas ejected by the galaxy is transported to the outer halo primarily as a consequence of buoyancy rather than because of its velocity (Bower et al. 2017).

The expectation that the outflowing molecular clouds may dissolve into the warm/hot ionized phase has been inferred from various studies (e.g. Scannapieco and Brüggen 2015; Ferrara et al. 2016) in which the outflowing molecular clouds, subject to ram or radiation pressure, should undergo Kelvin-Helmholtz or Rayleigh-Taylor instabilities, and dissolve on timescales shorter than the outflow dynamical time (see Sect. 2.3.1 for more details). Moreover, photoevaporation has been identified as an additional process that may erode outflowing molecular clouds to the point that they can completely dissolve (e.g. Decataldo et al. 2017).

Direct observational evidence for gas phase transition from molecular to neutralatomic or from neutral-atomic to warm/hot ionized is rare. Arguably the best case for molecular to neutral-atomic transition is that of the increasing neutral-to-molecular gas fraction with increasing heights above the disk in the nearby system $\mathrm{M} 82$, where the linear resolution is only $\sim 30 \mathrm{pc} \operatorname{arcsec}^{-1}$ (Sect. 4.2.1; Leroy et al. 2015a). In contrast, NGC 253, an object at basically the same distance as M 82 and with data of similar quality, does not show any obvious gas phase transitions in the outflowing gas (Sect. 4.2.2; Bolatto et al. 2013a; Walter et al. 2017). The similarity of the velocity profiles of Si II, Si III, Si IV, and O VI in nearby starbursts also does not support phase transition within the inner $\sim$ kpc of these outflows. Detailed multi-phase comparisons have been conducted in a few, more distant infrared-bright galaxies using 3D data on the outflowing molecular, neutral-atomic, and ionized gas phases with matched sub-arcsecond resolutions, but $\sim 10 \times$ poorer linear resolution than for M 82 and NGC 253 (e.g., Rupke and Veilleux 2013b; Rupke et al. 2017). The conclusions 
of these comparisons have been mixed. In some cases, the gas phases overlap spatially and kinematically with each other, suggesting that they move together as a single entity without mixing or phase transition. In other cases, the cold molecular gas is slower and more concentrated in the nucleus than the neutral-atomic component, and the ionized gas is faster on average than the neutral-atomic gas and also more diffuse and extended (Rupke et al. 2017). The observational evidence for phase transitions on CGM scales is even weaker since most of the information on these scales comes from transverse spectroscopic studies of background quasars that do not spatially sample the absorbers. The use of galaxies as background sources is slowly changing this and promises to map cloud structures of different phases (Rubin et al. 2018a,b).

While some of the models mentioned above predict the molecular clouds to dissolve in the harsh environment of outflows, other models predict the molecular clouds to not only survive but to actually cool even further, evolving in even denser molecular phases (Sect. 2.3.2, e.g. Richings and Faucher-Giguère 2018b; Zubovas and King 2014; Decataldo et al. 2019; Gronke and Oh 2019). In some of these models, the expectation is that the molecular clouds should become gravitationally unstable, fragment, and form stars inside the outflow (Zubovas et al. 2013; Zubovas and King 2014; Ishibashi and Fabian 2012; Ishibashi et al. 2013, 2017; El-Badry et al. 2016; Wang and Loeb 2018; Decataldo et al. 2019). In these models, the expected star formation inside the outflow could potentially reach hundreds of solar masses per year. This new mode of star formation would have remarkable consequences. Indeed, once formed in the outflow, the stars would have the kinematic imprint of the outflowing molecular gas out of which they have formed, and would start moving ballistically on quasi-radial orbits. If their velocity does not exceed the escape velocity, then they would start oscillating around the galactic center, hence contributing to the spheroidal component of galaxies (bulge, halo, or possibly even contributing to the formation of elliptical galaxies). High velocity stars, stellar shells, and intracluster supernovae are some of the other properties that could be explained by this scenario, according to some authors. Finding observational evidence for star formation in galactic outflows is not simple as the signature of star formation can be easily overwhelmed by the presence of shocks and, even more likely, by the AGN photoionization (Gallagher et al. 2019; Hinkle et al. 2019). Moreover, young stars formed in the outflow may be difficult to disentangle from star formation in the host galaxy; indeed, as soon as stars form in the galactic clouds they respond only to gravity and are therefore expected to be rapidly decelerated to velocities that are hardly distinguishable from the stars in the host galaxy (Maiolino et al. 2017).

Despite these issues, recent studies have presented observational indication of star formation in some galactic outflows. Maiolino et al. (2017) have investigated in detail the outflow in the ULIRG F23128-5919 and found that the both optical and near-IR nebular diagnostics of the outflowing gas are consistent with star formation. A similar analysis was extended by Gallagher et al. (2019) and by Rodríguez del Pino et al. (2019) to samples of tens of local galaxies by exploiting the MaNGA integral field spectroscopic survey. About one third of the outflows have nebular line diagnostics consistent with excitation by star formation. The question is whether the excitation is due to star formation inside the outflow or externally from star formation in the disc of the host galaxy. The ionization parameter $\left(U_{H} \propto r^{-2} n_{H}^{-1}\right.$; Eq. 40) should be 
different in the two cases. Indeed, in the case of external photoionization (e.g., young stars in the host galaxy), the ionization parameter should be lower in the outflowing gas, primarily because the gas is located at larger distances from the ionizing source, which is not compensated by a decrease in the gas density (as discussed in Sect. 5.2, the density of the gas in the outflows is actually systematically higher than in the disk). The finding that in those outflows with SF-like diagnostics the ionization parameter is similar, or even higher, than in their host galaxies favour the scenario in which star formation is occurring in situ, i.e. inside the outflow, therefore bringing support to the various theoretical models discussed above.

Assessing the occurrence of this phenomenon is still difficult and sometimes controversial. Based on scaling relations, Gallagher et al. (2019) suggest that the phenomenon of star formation inside outflows may be even more prominent in more powerful and more massive outflows typically occurring in the more active phases at high redshift. However, the most powerful winds are driven by quasars, which can easily overwhelm any star-forming diagnostic in the outflow, even if the SFR in the outflow is relatively high (see more detailed, quantitative discussion in Gallagher et al. 2019). Hinkle et al. (2019) investigate the ionization properties of four PG quasars and find that they are indeed dominated by AGN-like or shock-like excitation. They do find a region of one of the outflows dominated by SF-like excitation, but ascribe it to external photoionization based on the proximity, in projection, with star-forming regions in the host galaxy. This result underlines the need to accurately model the 3D position of the outflowing gas with respect to all of the star-forming regions in this object and all other galaxies with suspected in situ star formation.

If star formation inside outflows is a channel contributing to the formation of the spheroidal component of galaxies, one may wonder whether the Milky Way's halo should show the signatures of such stars formed in past outflows. Within this context it is interesting that, by exploiting the latest GAIA data release, Belokurov et al. (2019) have recently discovered a significant population of metal-rich halo stars with purely radial orbits, i.e. with the same properties expected by models of stars formed in galactic outflows (see e.g. sect. 4.1 of Zubovas et al. 2013). Indeed Belokurov et al. (2019) suggest that this might be one of the possible scenarios for the formation of such stars. It is also interesting that, according to their age, all these metal-rich MW halo stars with radial orbits were formed between $\mathrm{z} \sim 1$ and $\mathrm{z} \sim 4$, which is when the higher central star formation and AGN activity is expected to have generated the strongest outflows.

It should be noted that there are several other instances of "positive feedback" in galaxies in which outflows or jets boost star formation in galaxies by compressing their ISM or CGM. Beautiful, clear examples of this phenomenon are seen in Cen A (Sec. 4.5.1; Crockett et al. 2012; Santoro et al. 2016) and, thanks to new observing techniques (especially thanks to the new generation of wide-field IFS) more examples of outflow/jet-induced star formation occurring in other nearby galaxies are being found (e.g. Croft et al. 2006; Elbaz et al. 2009; Cresci et al. 2015; Shin et al. 2019). However, these are cases in which the star formation is triggered in the host galaxy by the compression of the outflow/jet and the newly formed stars tend to preserve the kinematic properties of their host galaxy, hence a process fundamentally different from the star formation inside outflows discussed above. While these find- 
ings support several recent models of jet/outflow-induced star formation (Mukherjee et al. 2018a; Gaibler et al. 2012; Silk 2013; Bieri et al. 2015, 2016; Dugan et al. 2017; Nayakshin and Zubovas 2012; Silk 2017), as well as older models predating all of these observations (e.g., De Young 1981), the inferred star formation rates associated with this mode are typically low (generally less than $0.1-1 M_{\odot} \mathrm{yr}^{-1}$ ), hence they probably do not have a major impact on the overall evolution of galaxies. However, recent evidence for positive feedback associated with the outflow of a powerful type 2 quasar at $z \sim 1.5$ has been detected, and in this case the inferred induced star formation rate is about $200 M_{\odot} \mathrm{yr}^{-1}$. Moreover, star formation induced by the radio jets of high-z radio galaxies has been known for a long time (McCarthy 1993; Cimatti et al. 1998; di Serego Alighieri et al. 1997; Feain et al. 2007). Therefore, the effect of star formation induced by outflows and jets may be important in the early Universe although its relevance relative to other mechanisms of galaxy formation has yet to be assessed properly.

\section{Cool Outflows in the Distant Universe}

\subsection{Molecular Gas Component}

While the detection of ionized outflows at high redshift has been obtained for large samples of star-forming galaxies and AGNs (e.g. Steidel et al. 2010; Genzel et al. 2014; Cano-Díaz et al. 2012; Carniani et al. 2015, 2017; Harrison 2017; Förster Schreiber et al. 2019), the detection of cold molecular outflows in high-redshift galaxies is still limited to a handful of objects, due to the observational difficulty in detecting the weak signatures of outflows in these distant systems and also to disentangle them from the dynamically quiescent ISM in the host galaxies. Moreover, even when detected, the limited projected spatial resolution often prevents a proper assessment of the extension and geometry of the outflow.

High-sensitivity observations of $\mathrm{CO}$ transitions have revealed high-velocity molecular gas ascribed to outflows in about half a dozen high-redshift galaxies (Fig. 30; Weiß et al. 2012; Feruglio et al. 2017; Carniani et al. 2017; Vayner et al. 2017; Fan et al. 2018; Brusa et al. 2018; Herrera-Camus et al. 2019c), although in some of these it is not yet totally clear whether the high-velocity molecular gas is truly associated with outflow or actually result from merging or irregular dynamics. In all of these cases the outflow is driven by an AGN, typically a powerful quasar, but also sometimes a lower luminosity AGN residing in a normal main-sequence galaxy (Fig 30e, Herrera-Camus et al. 2019c), and also molecular outflows driven by radio-jets (Vayner et al. 2017). These molecular outflows in distant galaxies have velocities up to $1000 \mathrm{~km} \mathrm{~s}^{-1}$ and extend from a few kpc to several kpc.

The detection of molecular outflows through blueshifted far-IR molecular transitions in absorption (redshifted to the millimeter and submillimeter bands, observable from the ground) has been limited largely to extremely powerful starburst galaxies, which are also strongly lensed, as the detection of these features in absorption requires a strong continuum (typically in excess of $100 \mathrm{mJy}$ ). The only exception is quasar J131911+095051 (Herrera-Camus et al. 2019b). There are currently only four 

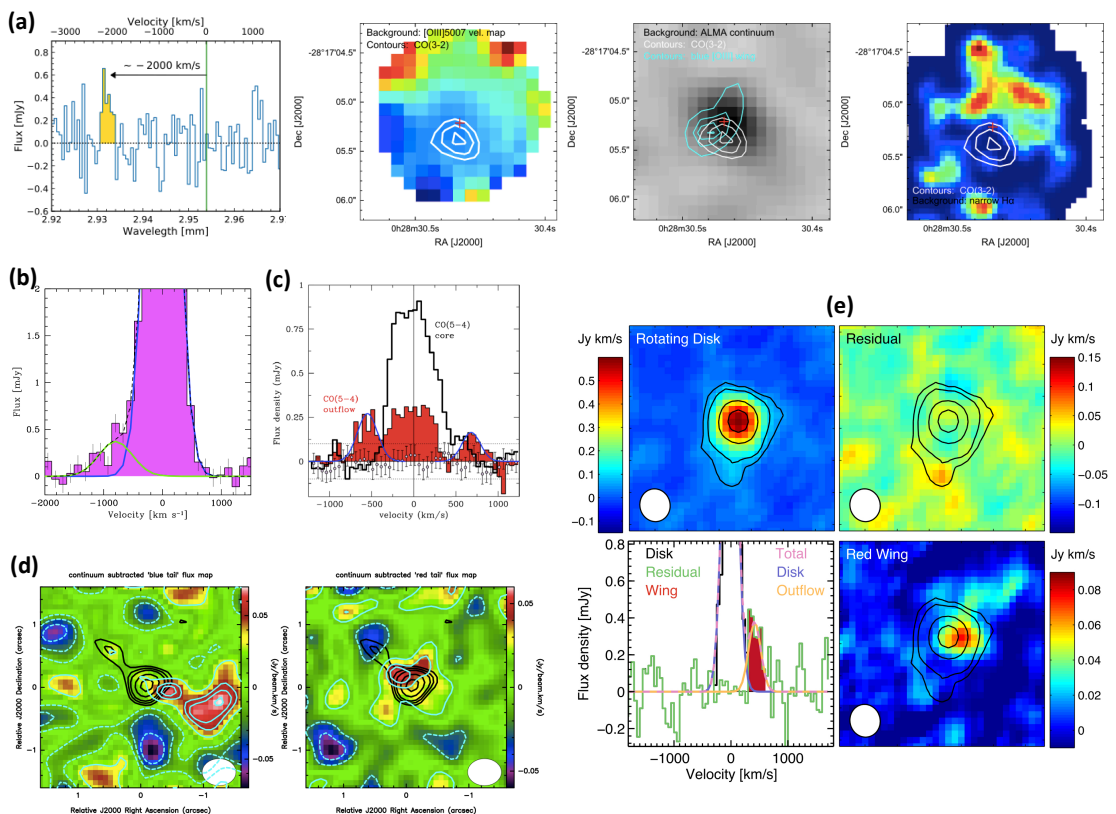

Fig. 30 Examples of molecular outflows in distant galaxies detected via emission lines. (a) From left to right: blueshifted CO (3-2) line emission from $z=2.4$ quasar 2QZJ0028, flux map of the blueshifted CO (3-2) line emission (white contours) overlaid on the [O III] velocity map, [O III] blue wing (blue contours), ALMA 3-mm continuum map (greyscale), and narrow $\mathrm{H} \alpha$ emission (color scale) tracing star formation in the host galaxy. (b) Asymmetric CO (4-3) line emission from $z=3.9$ quasar APM 08279+5255. (c) CO (5-4) outflow (red histogram) and core (black histogram) in quasar XID2028. (d) Maps of the blueshifted $\left(v<-350 \mathrm{~km} \mathrm{~s}^{-1}\right.$; left panel) and redshifted $\left(v>350 \mathrm{~km} \mathrm{~s}^{-1}\right.$; right panel) CO (5-4) line emission shown in panel (c), color-coded according to the flux. (e) AGN-driven CO (3-2) outflow in the massive main-sequence galaxy zC400528 at $z=2.3$. The emission maps of the three components identified in the integrated spectrum shown in the lower left panel are presented in the three other panels. Images reproduced with permission from (a) Carniani et al. (2017), (b) Feruglio et al. (2017), (c, d) Brusa et al. (2018), copyright by ESO; and (e) Herrera-Camus et al. (2019c), copyright by AAS.

outflow detections, from redshift 2.3 to 6.1 (Fig. 31), three of these obtained through the detection of $\mathrm{OH}$ transitions (George et al. 2014; Spilker et al. 2018; HerreraCamus et al. 2019b) and one of them through the detection of blueshifted high-level transitions of water (Jones et al. 2019a). The latter is a particularly interesting result, as it implies very dense $\left(\sim 10^{5}-10^{6} \mathrm{~cm}^{-3}\right)$ and warm $(300-500 \mathrm{~K})$ molecular outflowing gas, with large column densities $\left(\sim 10^{24} \mathrm{~cm}^{-2}\right.$ or even higher).

The fact that the high-z molecular outflows are detected only in AGN/quasar host galaxies when searching for $\mathrm{CO}$ high-velocity emission, while outflows are detected almost exclusively in starburst galaxies when searching for blueshifted far-IR $\mathrm{OH}$ or $\mathrm{H}_{2} \mathrm{O}$ absorption, does not really have any underlying, intrinsic physical reason. The reason for this dichotomy is most likely a consequence of observational selection effects. Indeed, on the one hand AGN-driven molecular outflows tend to be more vigorous (in terms of mass, outflow rate and kinetic power) and to have higher velocities with respect to starburst-driven outflows, hence easier to detect in 


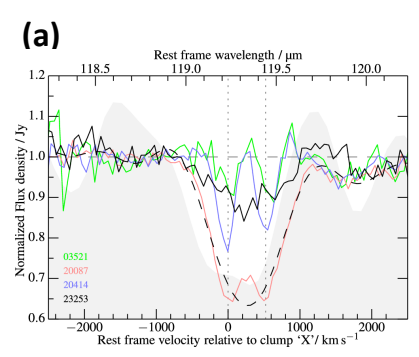

(b)

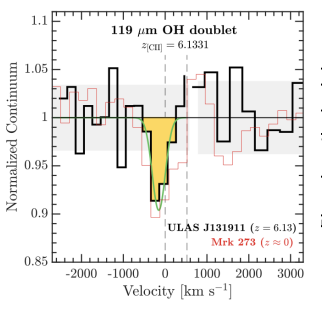

(c)

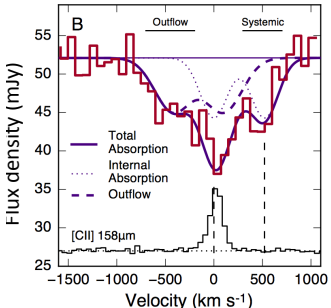

(d)

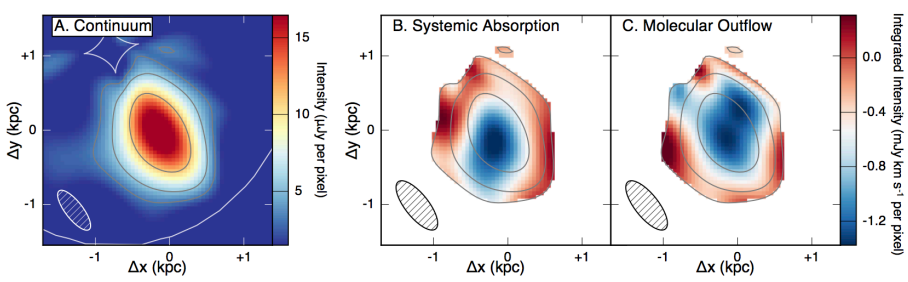

(e)

$\mathbf{N}$

(f)

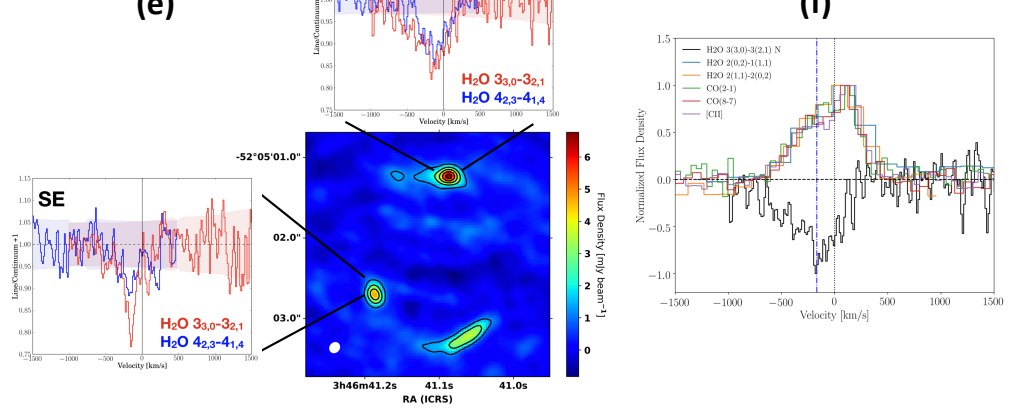

Fig. 31 Molecular outflows in distant galaxies detected via absorption lines. (a) Normalized OH 119 absorption profile of $z=2.3$ ULIRG SMM J2135-0102 (in grey) compared to those of nearby starburstdominated ULIRGs. (b) Normalized OH 119 absorption profile of $z=6.13$ quasar ULAS J131911+095051 compared with that of local ULIRG Mrk 273. (c) OH 119 absorption profile of $z=5.3$ lensed ULIRG SPT J231921-5557.9 compared with the profile of the [C II] $158 \mu$ m emission line. (d) Lensing reconstruction of the continuum emission (left), systemic $\mathrm{OH}$ absorption (middle), and $\mathrm{OH}$ outflow (right) in the object shown in panel (b). (e) $\mathrm{H}_{2} \mathrm{O}$ absorption spectra extracted at two locations in the continuum map of the lensed starburst ULIRG SPT-S J034640-5204.9. (f) Water absorption profile for the northern lensed image in panel (d) compared with the profiles of the [C II] $158 \mu \mathrm{m}, \mathrm{CO}(2-1)$, and two different water emission lines. Also shown is the two-Gaussian fit to the $\mathrm{H}_{2} \mathrm{O}$ absorption spectrum. Images reproduced with permission from (a) George et al. (2014), copyright by the authors; (b) Herrera-Camus et al. (2019c), copyright by ESO; (d) Spilker et al. (2018), copyright by the authors; (e, f) Jones et al. (2019a), copyright by ESO.

the sensitivity-limited $\mathrm{CO}$ searches of outflows at high-z. On the other hand, the detection of blueshifted $\mathrm{OH}$ and $\mathrm{H}_{2} \mathrm{O}$ absorption at high-z requires strongly lensed galaxies with strong far-IR continuum, and there are statistically many more lensed starburst galaxies with these characteristics (typically detected by the SPT and Herschel surveys) than lensed quasars. 
Overall, the quantitative findings of these high-redshift studies illustrate that the properties of the molecular outflows in distant galaxies follow similar trends as local galaxies, although the outflow properties seem to saturate at the more extreme and powerful tail (Fig. 32). More specifically, high-redshift star forming galaxies are characterized by a mass loading factor of about unity, as found for their local counterparts. However, for the most extreme starbursts found at high redshift, this implies outflow rates even exceeding $1000 \mathrm{M}_{\odot} \mathrm{yr}^{-1}$. For instance, the most extreme galaxy for which the molecular outflow has been detected (the lensed galaxy SPT-S J034640-5204.9 at $\mathrm{z}=5.6$; Fig. $31 \mathrm{~d}-\mathrm{e}$ ) is characterized by an extremely powerful and extremely compact starburst, resulting in an impressive surface density of star formation rate of $10,000 \mathrm{M}_{\odot} \mathrm{yr}^{-1} \mathrm{kpc}^{-2}$, an order of magnitude larger than the so-called "maximal starburst", i.e. the maximum Eddington-limited star formation surface density beyond which radiation pressure is expected to rapidly blow away the ISM (Thompson et al. 2005; Crocker et al. 2018). This galaxy is clearly in a highly transient phase. The outflow rate inferred from the water P-Cygni profile is only a few hundred $\mathrm{M}_{\odot} \mathrm{yr}^{-1}$, much lower than the star formation rate in the same galaxy, $3000 \mathrm{M}_{\odot} \mathrm{yr}^{-1}$, implying an outflow mass loading factor much less than unity, i.e. much lower than observed for low-z galaxies (it is the rightmost data point in Fig. 32). Apparently, being so highly super-Eddington, the outflow capacity has saturated; in this case the outflow cannot regulate the star formation rate (as in less extreme, low-z galaxies) and result into a runaway process in which star formation deplete gas in a few Myr, likely resulting into a compact massive quiescent galaxy, unless replenished with fresh gas from the IGM (Jones et al. 2019a). The second most extreme galaxy among those with blueshifted molecular absorption detection (another SPT lensed galaxy at $z=$ 5.3; Fig. 31b-c) displays similar properties, i.e. a very compact starburst (although not as extreme as SPT-S J034640-5204.9) and a low loading factor, although the latter is still consistent with unity within the uncertainties (it is the second data point from the right in Fig. 32).

Even in these high-z systems, the presence of a powerful AGN does boost the outflow rate by a significant factor, as in local systems, therefore confirming the important role of AGNs, and especially powerful quasars, in regulating or even quenching star formation in galaxies (Fig. 32). The energetics of these quasar-driven outflows at high- $\mathrm{z}$ are typically well below the $\sim 5 \%$ of $\mathrm{L}_{A G N}$ expected by the blast-wave, energydriven scenario (e.g., Bischetti et al. 2018). The momentum boost of these outflows also seems generally well below the factor expected by the energy-driven scenario (i.e. typically of the order of $\mathrm{L}_{\mathrm{AGN}} / \mathrm{c}$ or a few times $\mathrm{L}_{\mathrm{AGN}} / \mathrm{c}$, in contrast to the expectation of $>10 \mathrm{~L}_{A G N} / \mathrm{c}$ expected in the energy-driven scenario; Feruglio et al. 2017; Bischetti et al. 2018). These findings suggest that, as expected by numerical simulations, in these early systems the bulk of the hot nuclear wind does not couple efficiently with the galaxy ISM and mostly escapes into the IGM (Costa et al. 2015). Alternatively, a significant fraction of the outflow may be accelerated by radiation pressure, which results in a much lower momentum boost (e.g. Costa et al. 2018a; Ishibashi et al. 2018a). 


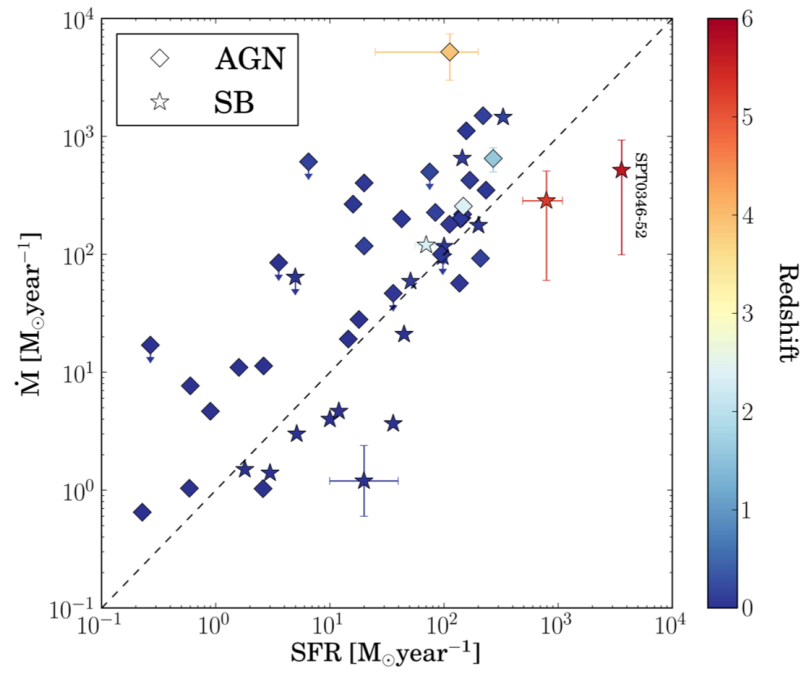

Fig. 32 Molecular mass outflow rates in distant galaxies plotted as function of star formation rates. The dashed line shows the 1:1 equality line. The symbols differentiate between AGN and starbursts and are color-coded by redshifts (Jones et al. 2019a), copyright by ESO.

\subsection{Neutral Atomic Gas Component}

There are many more reported cases of neutral-atomic outflows at high redshifts than molecular outflows. Down-the-barrel and transverse spectroscopic surveys of neutral/low-ionization UV absorption lines (C II, O I, Al II, Si II, and Fe II in absorption) and emission lines ( $\mathrm{Si} \mathrm{II}^{*}$, especially common at $z \sim 4$ ) from $z \gtrsim 2$ mainsequence star-forming galaxies have revealed ubiquitous, fast $\left(v_{50} \simeq 100-300 \mathrm{~km}\right.$ $\mathrm{s}^{-1}, v_{98}$ up to $\sim 1000 \mathrm{~km} \mathrm{~s}^{-1}$ ), neutral-atomic outflows extending to at least $\sim 50-100$ kpc ( 0.5 - $1 r_{\text {vir }}$; e.g., Shapley et al. 2003; Adelberger et al. 2005; Erb et al. 2012; Fox et al. 2008; Steidel et al. 2010; Rudie et al. 2012, 2019; Sugahara et al. 2017, 2019; Du et al. 2018; Gatkine et al. 2019). The outflow velocities tend to increase with redshifts up to $z \sim 2$, above which they seem to level off (Fig. 33, Sugahara et al. 2017, 2019; Du et al. 2018), as predicted by some models (e.g., Muratov et al. 2015). This turning point at $z \sim 2$ may also be due to a systematically higher SFR surface density in galaxies at $z \gtrsim 2$ (due to selection biases) or a change in outflow geometry (a more spherical outflow geometry at high $z$ will boost the detection fraction of outflows and increase the average/median outflow velocities in the stacked galaxy spectra).

In most cases, these results rely on the measurements of the strongest interstellar lines, which are typically saturated and therefore only trace the covering fraction and kinematics of the outflowing gas but not the column density (mass). Over the years, down-the-barrel studies of a total of $\sim 20$ gravitationally lensed systems at $z \simeq 2-4$ have addressed this weakness by measuring the fainter unsaturated lines of several elements (from H to $\mathrm{Zn}$ ) in a variety of ionization stages (Pettini et al. 2002; Quider 

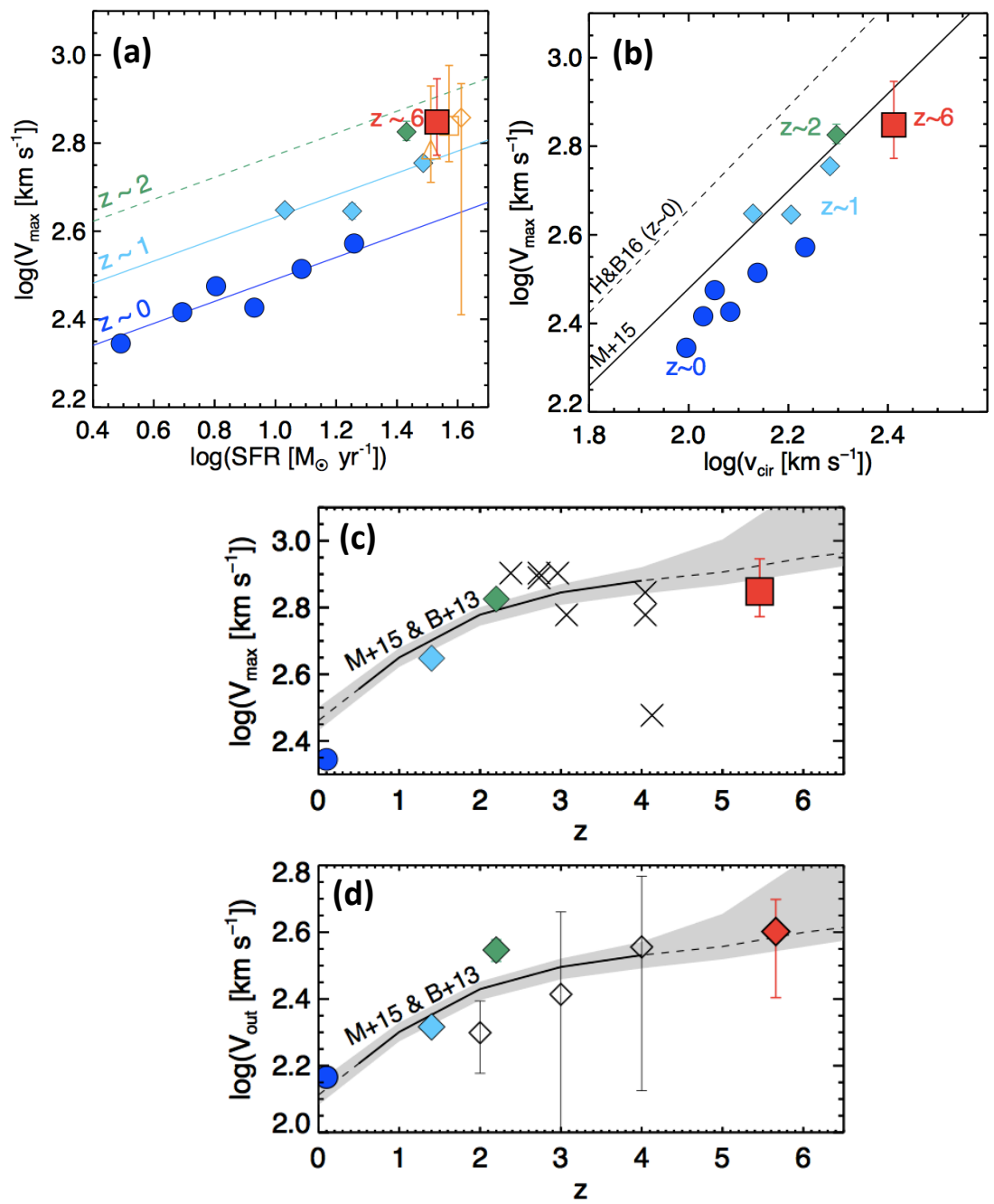

Fig. 33 Neutral-atomic outflows in distant star-forming galaxies. Trends of increasing maximum outflow velocities with (a) SFR, (b) circular velocity and (a)-(d) redshift are present in the data. Images reproduced with permission from Sugahara et al. (2019), copyright by AAS.

et al. 2009; Dessauges-Zavadsky et al. 2010; Stark et al. 2013; Rigby et al. 2018b,a; Jones et al. 2018). These studies have confirmed the ubiquity of fast low-ionization outflows in high- $z$ main-sequence star-forming galaxies, and reported mass outflow rates of the low-ionization gas alone that typically match or exceed the star formation rates.

The presence of powerful AGN at the center of star-forming galaxies likely enhances the velocity of cool outflows on small scales (Talia et al. 2017), although in some cases the AGN photoionize the cool gas that is located too close from the center 
(a)
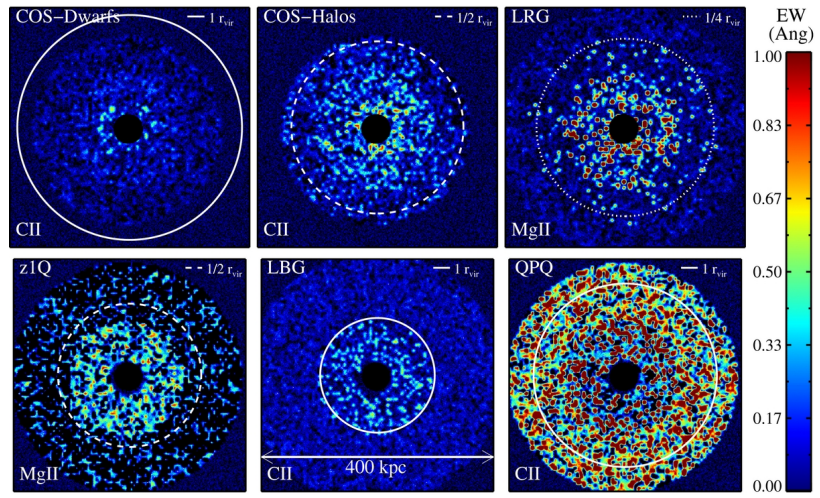

(b)

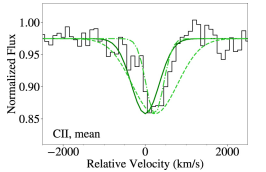

(d)

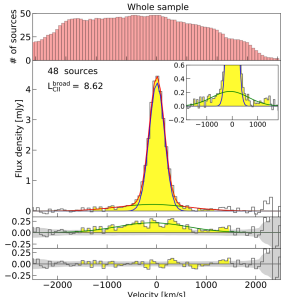

(c)

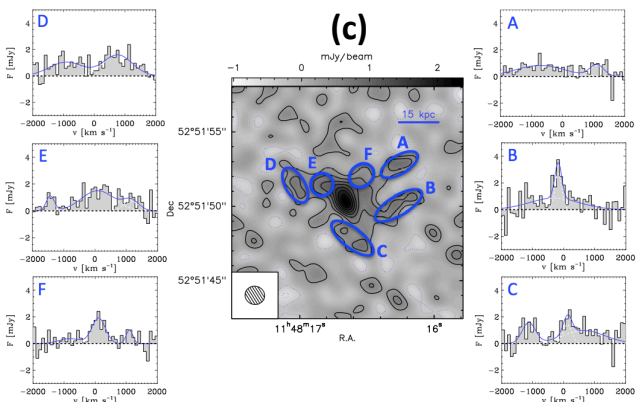

(e)

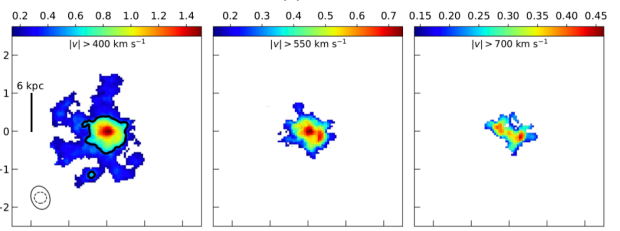

Fig. 34 Neutral-atomic outflows in distant quasars. (a) UV C II 1334 and Mg II 2796 absorbers around $z \simeq 2$ massive galaxies hosting quasars (lower-right panel) compared with those of $z \simeq 0 \mathrm{dwarf}$ and luminous galaxies (COS-Dwarfs and COS-Halos, respectively), $z \simeq 1$ Luminous Red Galaxies (LRG), $z \simeq 1$ quasars (z1Q), and $z \simeq 2$ Lyman-Break Galaxies (LBG). (b) Velocity shift between the mean stack of $\mathrm{C}$ II absorbers around $z \simeq 2$ quasars (black histogram) and the expected kinematics purely from clustering (solid green line). The dashed and dashed-dotted lines are absorption profiles centered on the data but with $\sigma_{v}=554$ and $214 \mathrm{~km} \mathrm{~s}^{-1}$, respectively. (c) Spatially resolved [C II] $158 \mu \mathrm{m}$ outflow around the quasar SDSS J1148+5251. (d) Stacked integrated [C II] $158 \mu \mathrm{m}$ spectrum of 48 quasars at $4.5<z<$ 7.1. (e) Luminosity maps of the high-velocity [C II] emission derived from the spectrum in panel (d). Images reproduced with permission from (a) Prochaska et al. (2014), (b) Lau et al. (2018), copyright by AAS; and (c) Cicone et al. (2015), (d, e) Bischetti et al. (2018), copyright by ESO.

(Perrotta et al. 2016). In the most extreme cases of $z \simeq 2$ LoBALs and FeLoBALs, the outflowing low-ionization material reaches velocities of several $\times 1000 \mathrm{~km} \mathrm{~s}^{-1}$ on scales of 1-3 kpc (based on Eq. (40) and densities derived from $\mathrm{Fe} \mathrm{II}^{*} / \mathrm{Fe}$ II and $\mathrm{Si} \mathrm{II}^{*} / \mathrm{Si}$ II ratios; Moe et al. 2009; Dunn et al. 2010). The implied outflow mass rates and kinetic luminosities are a few $\times 100 \mathrm{M}_{\odot} \mathrm{yr}^{-1}$ and $0.1-1 \% L_{\mathrm{BOL}}$, respectively. 
On CGM scales, transverse studies of C II and Mg II absorbers around powerful $z \simeq 2$ quasars (Fig. 34a; Prochaska et al. 2014; Lau et al. 2016, 2018) have revealed large reservoirs $\left(\sim 2 \times 10^{11} M_{\odot}\right)$ of cool gas with $\sim 0.7$ covering fraction, $\sim 1 / 4$ solar metallicity, and super-solar $\mathrm{O} / \mathrm{Fe}$ ratios, indicative of enrichment primarily by corecollapse SNe. To avoid Fe dilution by SNe Ia, the metals must be injected into the CGM of these quasars in less than $\sim 1$ Gyr through a burst of star formation of $\gtrsim 50$ $\mathrm{M}_{\odot} \mathrm{yr}^{-1}$. Prochaska et al. (2014) have argued that the large amount of metals in the CGM of these quasars may simply reflect their large halo masses $\left(\gtrsim 10^{12.5} \mathrm{M}_{\odot}\right)$ rather than the influence of the quasars. Indeed, the broad velocity distribution of the C II absorbers can largely be explained by virial motion within the galactic halos. However, the observed velocity offset of $+200 \mathrm{~km} \mathrm{~s}^{-1}$ of the cool absorbing system with respect to systemic velocity (Fig. 34b) seems to require a net outflow of $\sim 400-450$ $\mathrm{km} \mathrm{s}^{-1}$, combined with anisotropic and/or intermittent ionizing radiation from the quasars (Lau et al. 2018).

Photoionization by internal or external energy sources is also an important factor when studying the cool CGM in line emission. For instance, CGM-scale Ly $\alpha$ nebulae have been detected around several high- $z$ star-forming and active galaxies through stacking analyses (e.g., Steidel et al. 2011; Momose et al. 2016) and deep integral-field spectroscopy (e.g., Borisova et al. 2016; Wisotzki et al. 2016, 2018; Erb et al. 2018; Arrigoni Battaia et al. 2019; Cai et al. 2019; O'Sullivan et al. 2019), but in most cases, these nebulae are photoionized by the central quasars or starbursts (e.g., Leibler et al. 2018) or the cosmic UV background (e.g., Wisotzki et al. 2018), and thus they trace largely the warm-ionized component of the CGM rather the cool neutral-atomic gas phase. Moreover, the severe radiative transfer effects of this strong resonant line complicate the interpretation of the broad Ly $\alpha$ line profiles, making this line an ambiguous kinematic tracer of outflows in general (Sec. 3.3.2). Much of the recent effort has instead been directed at mapping the high- $z$ CGM using cool-gas tracers that are not affected by these effects.

Being one of the brightest lines in the spectrum of galaxies, the [C II] $158 \mu \mathrm{m}$ transition has been extensively used to search for outflows in galaxies at $z>4$, i.e. at redshifts high enough that this transition is shifted to the submm/mm-wave bands with good atmospheric transmission. As discussed in Section 3.4.4, this transition is expected to trace predominantly the atomic neutral component of the ISM and outflows, although contribution from the ionized phase may be significant. [C II] is currently detected in a few hundred galaxies at high redshift, however, as for $\mathrm{CO}$, the detection of the outflow component has been much more difficult, as it implies detecting broad wings, typically much fainter than the core of the line, and often distributed in a more diffuse component. The most prominent detection of cold atomic outflow at high-z has been obtained for the famous quasar J1148+5251 at $z=6.4$ (Fig. 34c; Maiolino et al. 2012; Cicone et al. 2015), which was observed for over 30 hours with NOEMA, and where [C II] is detected out to velocities in excess of 1000 $\mathrm{km} \mathrm{s}^{-1}$, extending on scales of $30 \mathrm{kpc}$, matching expectations from zoom-in cosmological simulations and theoretical models specifically aimed at intepreting feedback in this quasar (Valiante et al. 2012; Costa et al. 2015). In this case the outflow has been mapped in such detail that it was possible to infer the geometry and temporal evolution of the outflow. More specifically, the outflow appears to have a biconical 
structure along our line of sight; indeed detailed modelling (Costa et al. 2015) shows that this is the only geometry that can match the nearly symmetric, double-peaked distribution of the [C II] high-velocity component on large spatial scales. Moreover, the distribution of the dynamical timescale of the various outflowing clumps indicates that the outflow has been ongoing for at least $10^{8} \mathrm{yr}$ and in a bursty mode.

However, J1148+5251 seems to be an extreme case. Detections of atomic cold outflows through [C II] wings has so far been achieved only for a few additional individual quasars (Díaz-Santos et al. 2016, Carniani et al. in prep.). Yet, the combination of ALMA [C II] data of nearly 50 quasars at z 5-7 (corresponding a total of nearly 40 hours of integration) has revealed clear broad wings in the stacked cube (Fig. 34d-e; Bischetti et al. 2018), indicating that these primeval quasars on average drive highvelocity cold outflows as expected by models and cosmological simulations (e.g. Di Matteo et al. 2005; Valiante et al. 2012; Costa et al. 2015, 2018b). The stacked cube also suggests that these quasar-driven outflows have extensions of several kpc. Stacking analyses no doubt miss a significant fraction of faint outflows due to orientation effects (Stanley et al. 2019), so these results should be considered conservative lower limits.

Early attempts to detect cool winds in distant "normal" star-forming galaxies through the [C II] line and through stacking of 10-20 galaxies have resulted in tentative detections of broad wings (Gallerani et al. 2018; Fujimoto et al. 2019). A clearer and more definitive result has been obtained through the ALPINE ALMA large program, which has enabled the stacking of nearly 100 galaxies (Ginolfi et al. 2019). A clear detection of broad wings, extending to velocities of several $100 \mathrm{~km} \mathrm{~s}^{-1}$ and on kpc scales, is however obtained only when the stacking is restricted to the most star forming galaxies (SFR $>100 M_{\odot} \mathrm{yr}^{-1}$ ).

\subsection{Dust Component}

Direct evidence for dust in cool outflows based on the detection of spatially extended dust emission coincident with extended broad CO or [C II] line emission is rare (possibly seen only in SDSS J1148+5251; Cicone et al. 2015). The same is true for dust depletion in individual low-ionization outflows. In the few cases of $z \simeq 2-3$ gravitationally-lensed star-forming systems where this type of detailed down-thebarrel analysis is possible, the results are ambiguous. The outflowing gas often shows super-solar alpha-to-iron ratios consistent with dust depletion, but these anomalous ratios may also be explained if the bulk of the stars in these galaxies formed recently ( $\lesssim 0.3$ Gyr; Pettini et al. 2002; Dessauges-Zavadsky et al. 2010; Jones et al. 2018).

Arguably the most convincing argument for dust in cool outflows at $z \gtrsim 1$ comes from reddening measurements of quasars with intervening strong Mg II absorbers. Ménard and Fukugita (2012) have deduced that strong Mg II absorbers at $z=0.4-2.0$ carry about half of the total amount of dust outside of galaxies (see Sec. 5.3), or nearly all of the circumgalactic dust within the virial radii of galaxies. These absorbers have a constant MW-like dust-to-gas ratio that is consistent with an outflow origin, where dust is neither added through stellar processes nor destroyed by sputtering over the inferred long (several Gyr) effective lifetime of these clouds. 
Dust is also present in most $z \gtrsim 1.5$ DLAs with $N_{\mathrm{H}}>10^{20.3} \mathrm{~cm}^{-2}$ and sub-DLAs with $N_{\mathrm{H}}=10^{19-20.3} \mathrm{~cm}^{-2}$, based on background quasar reddening (Fukugita and Ménard 2015) and absorption-line measurements of differential elemental depletion (Wolfe et al. 2005; Dessauges-Zavadsky et al. 2006; Jenkins 2009; Kulkarni et al. 2015; Quiret et al. 2016; De Cia et al. 2016; De Cia 2018). However, these systems generally have $3-10 \times$ lower metallicity than that of $\mathrm{Mg}$ II absorbers and their dustto-gas ratio, metallicity, and line width all decrease with increasing $N_{H}$, contrary to that of Mg II absorbers. The metals in DLAs may have been ejected from galaxies in cool winds as with $\mathrm{Mg}$ II clouds, but this gas must have subsequently mixed with primordial gas on intergalactic scales to produce the observed metallicity and dustto-gas ratio, and their dependence on $N_{\mathrm{H}}$ (Fukugita and Ménard 2015). Note also that the MW-like $2175 \AA$ graphite feature is detected in some of these systems, but not in Mg II absorbers (Ma et al. 2017).

Most high-redshift AGN, particularly those with red or IR-bright SEDs, contain large quantities of dust, but a firm association between dust and outflows in these systems is still lacking. The case for entrained dust in LoBAL and FeLoBAL quasars has already been discussed in Section 5.3, when discussing low-redshift systems, so it is not repeated here. Many more quasars with very red optical-IR colors, remarkably blue or flat colors in the UV, and fast powerful warm-ionized winds seen in both line emission and absorption have been discovered by cross-correlating the sources in the WISE mid-infrared catalog of galaxies with those from other large ground-based extragalactic surveys. These so-called "extremely red quasars" or ERQs represent a promising new class of objects to search for dust in outflows (Banerji et al. 2015; Assef et al. 2015; Hamann et al. 2017; Perrotta et al. 2019; Coatman et al. 2019; Temple et al. 2019). A recent spectropolarimetric follow-up study of a small subset of these objects by Alexandroff et al. (2018) has revealed high polarization $(\sim 15 \%)$ in the UV continuum, indicating that most of the continuum emission at these wavelengths is due to anisotropic scattered light, most likely off of a dusty medium in proximity to the AGN. Alexandroff et al. (2018) have also argued that the strength and swing in position angle of the polarization across the profiles of the emission lines can most simply be explained with a geometrically thick equatorial dusty scattering outflows of several thousand $\mathrm{km} \mathrm{s}^{-1}$ that extends on scales of tens of parsecs. On the other hand, Temple et al. (2019) recently concluded that most of the dust in extremely red quasars lies on galactic scales outside of the ionized [O III] outflows. Clearly, the jury is still out on this issue, and the possibility that dust may be present on both nuclear and galactic scales, as in FeLoBALs, is not excluded.

\subsection{Fate of the Outflowing Material}

It is difficult to estimate the escape velocity in high-redshift systems. However, most of the molecular outflows detected at these high redshifts seem hosted in very massive galaxies, characterised by high SFR and high AGN power (this is simply a consequence of the current selection biases), so even velocities as high as $1,000 \mathrm{~km} \mathrm{~s}^{-1}$ would not enable the cold outflowing gas to escape the halo of these galaxies. Hence the gas is likely to rain back onto the host galaxy unless heated and prevented to cool. 
Nevertheless, it is interesting to note that quasar-driven outflows seem to have an immediate quenching effect on those regions of the host galaxy hit by the outflow: indeed in these regions star formation appears to be suppressed relative to other regions of the host galaxy (Cano-Díaz et al. 2012; Cresci et al. 2015; Carniani et al. 2016). However, overall the elevated star formation rate across the rest of the quasar galaxy seems unaffected, which has raised questions on the effectiveness of quasar-driven outflows in quenching star formation at high redshift (e.g. Balmaverde et al. 2016; Schulze et al. 2019). In fact, there are indications that, in some of these high-z galaxies characterized by quasar-driven outflows, star formation can actually be boosted as a consequence of gas compression in regions surrounding the fast outflow (Cresci et al. 2015; Carniani et al. 2016). In these cases, the net feedback effect seems to be more positive than negative.

The story for the outflowing neutral-atomic material is seemingly quite different. As mentioned in Sec. 6.2 and shown in Fig 33, the outflow velocities of the neutralatomic gas at $z \gtrsim 2$ appear to be larger on average than those measured at low redshifts. More importantly, the stellar masses of the high- $z$ host galaxies are typically 1-2 dex smaller than those at low redshifts (e.g., Behroozi et al. 2013), so their escape velocities are correspondingly 0.3-0.6 dex smaller (Sec. 2.4). As a result, the bulk of the cool outflowing material in these high- $z$ systems often has a large enough velocity to escape from the host galaxies altogether, contrary to the cases at low redshifts (Sec. $5.1,5.6)$. If unimpeded by drag forces, this outflowing neutral-atomic material will contribute to the enrichment of the CGM and IGM (e.g., Muratov et al. 2015, 2017), and may re-accrete onto neigboring galaxies (Anglés-Alcázar et al. 2017).

The difference between the molecular and atomic gas phases in high- $z$ outflows no doubt is due to selection biases rather than a physically meaningful difference between the phases. High- $z$ cold molecular and [C II]-based outflows have so far been detected only in massive galaxies, for which the escape velocity is very high, while the neutral outflowing phase observed through absorption line studies is mostly detected in lower mass main-sequence star-forming galaxies which have smaller escape velocities. So the current results reflect the different populations of galaxies being probed by different outflow tracers rather than differences in outflow phases.

\section{Open Issues and Future Directions}

\subsection{Theoretical Challenges}

\subsubsection{Wind-ISM Interaction}

Fundamentally, the issue here is whether cool ISM clouds entrained in a hot wind can reproduce the observed characteristics of cool outflows. The first basic question is whether ram-pressure forces exerted by a fast low-density wind on a dense $\left(\sim 10^{4}\right.$ $\left.\mathrm{cm}^{-3}\right)$, cold $(\sim 10-100 \mathrm{~K})$ cloud initially at rest can accelerate this cloud to $\sim 200$ (1000) $\mathrm{km} \mathrm{s}^{-1}$ over a scale of $\sim 200$ (1000) pc, without shredding it into pieces by erosion processes. These densities, velocities, and distance scales are representative of the outflowing molecular gas in NGC 253 and Mrk 231, respectively (Sect. 4.2.2 
and 4.4). The fast $\left(\sim 450 \mathrm{~km} \mathrm{~s}^{-1}\right)$ and very dense $\left(\sim 10^{7}-10^{5} \mathrm{~cm}^{-3}\right)$ clouds seen outflowing on parsec scale in NGC 1068 (Sect. 4.3) are equally if not more challenging to explain in this entrainment scenario. Recent high-resolution 2D and 3D simulations (McCourt et al. 2018; Sparre et al. 2019, respectively) of radiatively cooling clouds interacting with a supersonic, hot wind indicate that shattering / fragmentation of clouds larger than the cooling length is unavoidable. However, the quantitative differences between the results from these simulations ${ }^{7}$ underscore the need to strive for the most realistic state-of-the-art simulations and address the issue of numerical convergence to make substantial progress answering even this simple question. The importance of extending both the parameter space (e.g. $\left.t_{\mathrm{cool}, \mathrm{mix}} / t_{\mathrm{cc}}\right)$ and the simulation domain to follow up the evolution of the stripped material mixing with the hot fluid has also recently been emphasized by Gronke and Oh (2018, 2019, see Fig. 4). Other forces (e.g., radiation, cosmic rays, magnetic fields) may be added later to try to reproduce the substantial momentum boost $(\dot{p}>L / c)$ observed in energy-driven cool outflows, but the treatment of these other forces will require additional refinements to the simulations (see below) that are computationally costly and may compromise the accuracy of the overall results. Ultimately, the outcome of these simulations should be used to design observational diagnostics that distinguish between these various processes.

\subsubsection{Production and Survival of Dust and Molecules}

As discussed in Sec. 2.2.1 and 2.3.2, the molecular material in cool outflows may be produced in situ from the shocked ISM itself as long as the molecular gas manages to (re-)form within $\lesssim 10^{6}$ yrs. This short timescale favors $\mathrm{H}_{2}$ formation catalyzed by dust grains but it is at present unclear to what extent dust is able to survive the reverse and forward shocks produced by the violent interaction of the fast inner wind with the ISM (Sec. 3.5.6). In recent years, simulations and models of reprocessing of dust grains through shocks in SN remnant environments have shown that large $(a \gtrsim 0.1 \mu \mathrm{m})$ grains may survive or "escape" the shocks and decouple from the gas (Slavin et al. 2004; Silvia et al. 2010; Biscaro and Cherchneff 2016), but the results of these models do not necessarily apply to the more extreme conditions of coolgas outflows (characterized by higher shock velocities and stronger UV and X-ray radiation fields). Similarly, the failure of diffuse ejecta to reform the dust once the dust is destroyed by shocks (Biscaro and Cherchneff 2014) may not be relevant to the denser conditions of cool outflows. Moreover, dust growth in the more diffuse cold neutral medium may also be significant (Draine 2009; Ferrara et al. 2016; Zhukovska et al. 2016), but requires tracking the evolution of nano-grains in this lower density environment (since $t_{\mathrm{acc}} \propto a / n$; Eq. (60)). Modeling of the reprocessing (destruction, formation, and evolution) of both the dust and molecular gas needs to be done selfconsistently assuming the special time-dependent conditions of cool outflows to track

\footnotetext{
7 As pointed out by Sparre et al. (2019), instabilities have a smaller effect in 3D than in 2D because a $3 \mathrm{D}$ flow has the freedom to use the $z$-direction to avoid disturbing dense clouds. As a result, the level of fragmentation is lower in $3 \mathrm{D}$ than in $2 \mathrm{D}$, and the increase in covering fraction for large clouds in $3 \mathrm{D}$ is less than that seen in $2 \mathrm{D}$.
} 
the non-equilibrium molecular chemistry, and fully assess the validity of the scenario where the molecules form in situ within the wind.

\subsubsection{Coupling the Radiation to the Cool Gas}

Coupling of the radiation to the cool gas is inherently a difficult numerical problem since it requires in principle to resolve scales as small as the photon mean free path or dust destruction radius to capture all of the relevant physics, even in the simpler single-scattering optical thin limit (although promising workarounds have been developed in recent years; Krumholz 2018; Hopkins and Grudić 2019). In the optically thick limit, the numerical treatment of radiation Rayleigh-Taylor instabilities and frequency diffusion (due to $\kappa_{\mathrm{eff}, \mathrm{IR}} \propto v^{2}$ for IR radiation) is critically important and as a consequence considerable debate still remains regarding the efficacity of radiation to drive cool outflows (Sec. 2.2.2). Future numerical simulations that utilize the latest methods to solve the frequency-dependent radiative transfer equation and interpolate the solution back onto fluid elements will need to be tailor-made to reproduce the conditions of local U/LIRGs, where the most extreme momentum boosts $\dot{p} /(L / c)$ are observed, and should emphasize the distinguishing physical features of outflows accelerated by radiation pressure over those driven by thermal processes. Given the (tentative) evidence for elevated dust-to-gas ratios in the extraplanar material of some galaxies (Sec. 5.3) and decoupled dust/gas velocities in the ionized wind of M82 (Yoshida et al. 2011, 2019), the possibility of the dust grains decoupling dynamically from the gas due to the stronger radiation forces acting on them should also be investigated, keeping tract of the sizes and charges of dust grains and the collisions, Coulomb interactions, and Lorentz forces with the molecular, neutral-atomic, and ionized gas making up the outflow (Draine and Salpeter 1979b,a; Krumholz and Thompson 2013; Thompson et al. 2015).

\subsubsection{Importance of Cosmic Rays}

Tremendous progress have been made in recent years in modeling the effects of CRs as an additional source of pressure to drive warm and cool outflows (Sec. 2.2.3). The results are sensitive to the treatment of the microphysical processes associated with CR transport, e.g. relative importance of advection, streaming, and anisotropic diffusion of CRs along the magnetic fields. Future theoretical efforts should be directed at making quantitative morphological and kinematic comparisons between cosmic-ray driven outflows and those that do not include the effects of cosmic rays over a broad range of realistic initial ISM conditions (multi-phase density distribution, magnetic field strength and orientation), energy source distribution (SNe in a starburst, AGN), and detailed physics of the CR-plasma interaction. The importance of cosmic rays as a source of ionization of the cold molecular gas should also be investigated using the physical conditions appropriate to cold-outflow hosts (e.g., gas-rich U/LIRGs) to allow direct comparisons with the few existing observational constraints on partially ionized molecular outflows (e.g., Mrk 231; Sec. 4.4). 
7.2 Observational Challenges

\subsubsection{Unbiased Census of Local Molecular Outflows}

Current statistics on local molecular outflows are not representative, since they are highly biased in favor of special, highly active or gas-rich galaxies, such as ULIRGs, quasars, AGN, and starbursts where outflows may be brighter and easier to observe. The census of neutral atomic outflows also suffers from biases, but the situation is much better due to SDSS and large long-term investments of telescope time in optical and near-infrared spectroscopic surveys (Sect. 5.1). An unbiased census of local molecular outflows can in principle be carried out with current facilities (NOEMA, ALMA), given enough observing time. Future infrared and radio facilities (SPICA, the Origins Space Telescope, Square Kilometer Array, Next Generation VLA), however, have the sensitivity and throughput to significantly improve the picture. The Origins Space Telescope, for example, will be an efficient survey machine for low and high redshift (Bonato et al. 2019), capable of fast, very sensitive mid and farinfrared spectroscopy. As an interferometer, the Next Generation VLA (ngVLA) will provide high-resolution spectral imaging with very high sensitivity for the ground transitions of $\mathrm{CO}, \mathrm{HCN}$, and $\mathrm{HCO}^{+}$at $\lambda \sim 3 \mathrm{~mm}$, and several other powerful diagnostics of cold molecular outflows (Bolatto et al. 2018). The phase 1 mid-frequency version of the Square Kilometer Array (SKA), which should start construction soon, is optimizing for surveying at $\lambda \sim 21 \mathrm{~cm}$, and will be an extremely capable instrument to image outflows in neutral hydrogen emission and absorption (e.g., Morganti 2012).

\subsubsection{Outflow Physical Properties}

The measurements of the outflow physical properties, specifically mass loss rate, kinetic power, and momentum rate, are uncertain. The main issues are the geometry, velocity projection effect, and the determination of the mass in the outflow. There is a need for higher angular resolution and sensitivity (especially to diffuse low-surface brightness emission) to be able to apply several independent diagnostic tools on the same targets to reduce systematic errors and constrain the key quantities needed to calculate the energetics. Use of the fainter, higher level mm-wave CO, [C I], HCN, and $\mathrm{HCO}^{+}$transitions on a large sample of objects promises to provide a more complete picture of molecular outflows (especially a more accurate assessment of the gas mass in the outflow), while combined down-the-barrel and transverse multi-line restframe UV and optical absorption-line studies and deep 3D emission-line mapping of statistically significant samples of galaxies should help answer questions about inflight gas phase transition and gaseous extent of the neutral-atomic outflows. Soon, $J W S T$ will help probe the warm $\mathrm{H}_{2}$, predicted to be the dominant molecular component according to some quasar feedback models (Richings and Faucher-Giguère 2018b,a). Next-generation UV and X-ray missions will provide valuable constraints on the kinematics, ionization state, chemical composition, and dust content of the energetically important warm-hot and hot wind fluids, the "piston" in thermally driven cool outflows. The importance of CRs in driving these outflows will also be assessed 
quantitatively with LOFAR (e.g., Heesen et al. 2018, 2019; Mulcahy et al. 2018) and ngVLA.

\subsubsection{Negative Feedback: Zone of Influence and Escape Fraction}

The true impact of cool outflows can only be fully assessed by mapping the entire zone of influence of these outflows since negative feedback limited to small scales would not prevent the cool CGM from accreting back onto the galaxy host and forming new stars. Current absorption- and emission-line measurements are still severely limited by the sensitivity of current observations, particularly the surface brightness limits of emission-line mapping. On the short term, this can be improved by increasing the exposure times on a few key targets using large-format 3D spectroscopic instruments such as MUSE on the VLT and KCWI on Keck to provide full threedimensional coverage of the outflows on CGM scales and allow reliable decomposition of the outflowing material from the gravitationally bound material (e.g., Rupke et al. 2019). However, robust conclusions based on a statistically significant sample of galaxies will have to wait for the next generation of ground-based facilities including IFS on ELTs and ngVLA, and space-borne facilities such as SPICA, the Origins Space Telescope, LUVOIR, the Advanced Telescope for High-ENergy Astrophysics (Athena), and the Lynx X-ray telescope.

\subsubsection{Positive Feedback: Induced and In-Situ Star Formation}

The evidence for positive feedback is still sparse, even in the local universe. There is a need to carry out a systematic search for positive feedback in a statistically significant sample of objects. Actively star-forming hosts may need to be excluded from this sample to avoid any confusion with the source of ionization (internal vs external to the outflow), unless the geometry of the outflowing gas with respect to the host material is unambiguous. The technical challenge of disentangling the different sources of excitation in the gas (AGN, star formation, shocks) will still remain, but great strides have been made in recent years to resolve this problem by using the full suite of sophisticated photoionization and shock models in combination with kinematic predictions (Davies et al. 2014, 2016; Kewley et al. 2019). Yet, the fact that the presence of an AGN easily overwhelms the signature of star formation remains an issue difficult to overcome. Near-IR diagnostic nebular lines are promising alternatives to the classical line diagnostic diagrams in the optical (e.g. Maiolino et al. 2017) and are less affected by the AGN dominance; currently these near-IR diagnostics are observable with good $\mathrm{S} / \mathrm{N}$ only for a small number of outflows, but the sensitivity and resolution of JWST will enable to trace these diagnostics in essentially all local outflows and also at intermediate redshifts. The ultimate test will be directly detecting the signature of young stars and their kinematics; within this context deep observations with HST-COS or even with future facilities such as LUVOIR will help to unambiguously disentangle positive feedback phenomena. 


\subsubsection{Evolution with Look-Back Time}

Current observations indicate that warm-ionized and cool-atomic galactic winds are common at high redshifts, where main-sequence galaxies are very active and experiencing rapid growth. However, very little is known about their cold components, except in a handful of spectacular examples (e.g., Herrera-Camus et al. 2019c). Because of the biases and selection effects introduced by using different diagnostics to study the evolution of cool outflows with look-back time, it is preferable to use the same wind diagnostics at all redshifts. A fair comparison also requires matching linear resolution and flux limits of the observations. Ground-based 8-10 meter class OIR telescopes, ALMA, and VLA have already proven very useful to search for the rest-frame UV/optical, FIR, and mm-wave signatures of cool winds at $z \simeq 2-6$. JWST should soon extend our grasp to higher redshifts using optical/UV diagnostics without compromising on sensitivity and linear resolution. But large, sensitive, systematic surveys of molecular outflows reaching out to epochs where most galaxies are rapidly evolving are necessary to establish the importance of outflows in the cosmological context. Future facilities such as the ELTs, the ngVLA, SPICA, the Origins Space Telescope, and LUVOIR should be able to detect and characterize cool winds during the epoch of galaxy formation and fast growth of black hole seeds (e.g., GonzálezAlfonso et al. 2017a). Combining this together with studies of the growth of black holes at these redshifts, enabled by next-generation X-ray facilities such as Athena and Lynx, will shed new light on some of the key processes that shape galaxies.

Acknowledgements The authors thank the two referees of this review, particularly Mark Krumholz who provided a thorough report and made several constructive suggestions which improved the review. S.V. thanks the Editor, Joel Bregman, for the invitation to write this review. He thanks NASA for partial support of this research through NASA grant ADAP NNX16AF24G. S.V. also acknowledges support from a Raymond and Beverley Sackler Distinguished Visitor Fellowship and thanks the host institute, the Institute of Astronomy, where most of this review was written. He is also grateful for support by the Science and Technology Facilities Council (STFC) and by the Kavli Institute for Cosmology, Cambridge. S.V. also appreciates the hospitality of the Space Telescope Science Institute, where this review was completed. R.M. acknowledges ERC Advanced Grant 695671 'QUENCH' and support by the Science and Technology Facilities Council (STFC). A.B. acknowledges partial support from NSF-AST1615960. S. A. acknowledges funding from the European Research Council (ERC) under the European Union's Horizon 2020 research and innovation programme, grant agreement No ERC-2017-ADG-789410. S.A. also acknowledges the Swedish Research Council grant 621-2011-4143.

\section{References}

Aalto S (2015) Astrochemistry and star formation in nearby galaxies: from galaxy disks to hot nuclei. In: EAS Publications Series, vol 75-76, pp 73-80, DOI 10.1051/eas/1575013

Aalto S, Spaans M, Wiedner MC, Hüttemeister S (2007) Overluminous HNC line emission in Arp 220, NGC 4418 and Mrk 231. Global IR pumping or XDRs? A\&A464(1):193-200, DOI 10.1051/ 0004-6361:20066473, astro-ph/0612122

Aalto S, Costagliola F, van der Tak F, Meijerink R (2011) $\mathrm{H}_{3} \mathrm{O}^{+}$line emission from starbursts and AGNs. A\&A527:A69, DOI 10.1051/0004-6361/201015878, 1101.0682

Aalto S, Garcia-Burillo S, Muller S, Winters JM, van der Werf P, Henkel C, Costagliola F, Neri R (2012) Detection of $\mathrm{HCN}, \mathrm{HCO}^{+}$, and $\mathrm{HNC}$ in the Mrk 231 molecular outflow. Dense molecular gas in the AGN wind. A\&A537:A44, DOI 10.1051/0004-6361/201117919, 1111.6762 
Aalto S, Garcia-Burillo S, Muller S, Winters JM, Gonzalez-Alfonso E, van der Werf P, Henkel C, Costagliola F, Neri R (2015a) High resolution observations of $\mathrm{HCN}$ and $\mathrm{HCO}^{+} \mathrm{J}=3-2$ in the disk and outflow of Mrk 231. Detection of vibrationally excited HCN in the warped nucleus. A\&A574:A85, DOI 10.1051/0004-6361/201423987, 1411.2474

Aalto S, Martín S, Costagliola F, González-Alfonso E, Muller S, Sakamoto K, Fuller GA, García-Burillo S, van der Werf P, Neri R, Spaans M, Combes F, Viti S, Mühle S, Armus L, Evans A, Sturm E, Cernicharo J, Henkel C, Greve TR (2015b) Probing highly obscured, self-absorbed galaxy nuclei with vibrationally excited HCN. A\&A584:A42, DOI 10.1051/0004-6361/201526410, 1504.06824

Aalto S, Costagliola F, Muller S, Sakamoto K, Gallagher JS, Dasyra K, Wada K, Combes F, GarcíaBurillo S, Kristensen LE, Martín S, van der Werf P, Evans AS, Kotilainen J (2016) A precessing molecular jet signaling an obscured, growing supermassive black hole in NGC 1377? A\&A590:A73, DOI 10.1051/0004-6361/201527664, 1510.08827

Aalto S, Muller S, Costagliola F, Sakamoto K, Gallagher JS, Falstad N, König S, Dasyra K, Wada K, Combes F, García-Burillo S, Kristensen LE, Martín S, van der Werf P, Evans AS, Kotilainen J (2017) Luminous, pc-scale CO 6-5 emission in the obscured nucleus of NGC 1377. A\&A608:A22, DOI 10.1051/0004-6361/201730650, 1702.05458

Aalto S, Muller S, König S, Falstad N, Mangum J, Sakamoto K, Privon GC, Gallagher J, Combes F, García-Burillo S, Martín S, Viti S, van der Werf P, Evans AS, Black JH, Varenius E, Beswick R, Fuller G, Henkel C, Kohno K, Alatalo K, Mühle S (2019) The hidden heart of the luminous infrared galaxy IC 860. I. A molecular inflow feeding opaque, extreme nuclear activity. A\&A627:A147, DOI 10.1051/0004-6361/201935480, 1905.07275

Ackermann M, Albert A, Atwood WB, Baldini L, Ballet J, Barbiellini G, Bastieri D, Bellazzini R, Bissaldi E, Blandford RD, Bloom ED, Bottacini E, Brandt TJ, Bregeon J, Bruel P, Buehler R, Buson S, Caliandro GA, Cameron RA, Caragiulo M, Caraveo PA, Cavazzuti E, Cecchi C, Charles E, Chekhtman A, Chiang J, Chiaro G, Ciprini S, Claus R, Cohen-Tanugi J, Conrad J, Cutini S, D'Ammando F, de Angelis A, de Palma F, Dermer CD, Digel SW, Di Venere L, Silva EdCe, Drell PS, Favuzzi C, Ferrara EC, Focke WB, Franckowiak A, Fukazawa Y, Funk S, Fusco P, Gargano F, Gasparrini D, Germani S, Giglietto N, Giordano F, Giroletti M, Godfrey G, Gomez-Vargas GA, Grenier IA, Guiriec S, Hadasch D, Harding AK, Hays E, Hewitt JW, Hou X, Jogler T, Jóhannesson G, Johnson AS, Johnson WN, Kamae T, Kataoka J, Knödlseder J, Kocevski D, Kuss M, Larsson S, Latronico L, Longo F, Loparco F, Lovellette MN, Lubrano P, Malyshev D, Manfreda A, Massaro F, Mayer M, Mazziotta MN, McEnery JE, Michelson PF, Mitthumsiri W, Mizuno T, Monzani ME, Morselli A, Moskalenko IV, Murgia S, Nemmen R, Nuss E, Ohsugi T, Omodei N, Orienti M, Orlando E, Ormes JF, Paneque D, Panetta JH, Perkins JS, Pesce-Rollins M, Petrosian V, Piron F, Pivato G, Rainò S, Rando R, Razzano M, Razzaque S, Reimer A, Reimer O, Sánchez-Conde M, Schaal M, Schulz A, Sgrò C, Siskind EJ, Spandre G, Spinelli P, Stawarz Ł, Strong AW, Suson DJ, Tahara M, Takahashi H, Thayer JB, Tibaldo L, Tinivella M, Torres DF, Tosti G, Troja E, Uchiyama Y, Vianello G, Werner M, Winer BL, Wood KS, Wood M, Zaharijas G (2014) The Spectrum and Morphology of the Fermi Bubbles. ApJ793(1):64, DOI 10.1088/0004-637X/793/1/64, 1407.7905

Adelberger KL, Shapley AE, Steidel CC, Pettini M, Erb DK, Reddy NA (2005) The Connection between Galaxies and Intergalactic Absorption Lines at Redshift $2 \lesssim z \lesssim 3$. ApJ629(2):636-653, DOI 10. 1086/431753, astro-ph/0505122

Aditya JNHS, Kanekar N (2018) A Giant Metrewave Radio Telescope survey for associated H I $21 \mathrm{~cm}$ absorption in the Caltech-Jodrell flat-spectrum sample. MNRAS481(2):1578-1596, DOI 10.1093/ mnras/sty2184, 1808.03280

Alatalo K (2015) Escape, Accretion, or Star Formation? The Competing Depleters of Gas in the Quasar Markarian 231. ApJLett801(1):L17, DOI 10.1088/2041-8205/801/1/L17, 1502 .00624

Alatalo K, Blitz L, Young LM, Davis TA, Bureau M, Lopez LA, Cappellari M, Scott N, Shapiro KL, Crocker AF, Martín S, Bois M, Bournaud F, Davies RL, de Zeeuw PT, Duc PA, Emsellem E, FalcónBarroso J, Khochfar S, Krajnović D, Kuntschner H, Lablanche PY, McDermid RM, Morganti R, Naab T, Oosterloo T, Sarzi M, Serra P, Weijmans A (2011) Discovery of an Active Galactic Nucleus Driven Molecular Outflow in the Local Early-type Galaxy NGC 1266. ApJ735(2):88, DOI 10.1088/ 0004-637X/735/2/88, 1104.2326

Alatalo K, Lacy M, Lanz L, Bitsakis T, Appleton PN, Nyland K, Cales SL, Chang P, Davis TA, de Zeeuw PT, Lonsdale CJ, Martín S, Meier DS, Ogle PM (2015) Suppression of Star Formation in NGC 1266. ApJ798(1):31, DOI 10.1088/0004-637X/798/1/31, 1410.4556

Alatalo K, Cales SL, Rich JA, Appleton PN, Kewley LJ, Lacy M, Lanz L, Medling AM, Nyland K (2016) Shocked POststarbust Galaxy Survey. I. Candidate Post-starbust Galaxies with Emission Line Ratios 
Consistent with Shocks. ApJSupp.224(2):38, DOI 10.3847/0067-0049/224/2/38, 1601.05085

Alexandroff RM, Zakamska NL, Barth AJ, Hamann F, Strauss MA, Krolik J, Greene JE, Pâris I, Ross NP (2018) Spectropolarimetry of high-redshift obscured and red quasars. MNRAS479(4):4936-4957, DOI 10.1093/mnras/sty1685, 1806.10138

Anglés-Alcázar D, Faucher-Giguère CA, Quataert E, Hopkins PF, Feldmann R, Torrey P, Wetzel A, Kereš D (2017) Black holes on FIRE: stellar feedback limits early feeding of galactic nuclei. MNRAS472(1):L109-L114, DOI 10.1093/mnrasl/slx161, 1707.03832

Aoki K, Oyabu S, Dunn JP, Arav N, Edmonds D, Korista KT, Matsuhara H, Toba Y (2011) Outflow in Overlooked Luminous Quasar: Subaru Observations of AKARI J1757+5907. PASJ63:457, DOI 10.1093/pasj/63.sp2.S457, 1101.4340

Appleton PN, Diaz-Santos T, Fadda D, Ogle P, Togi A, Lanz L, Alatalo K, Fischer C, Rich J, Guillard P (2018) Jet-related Excitation of the [C II] Emission in the Active Galaxy NGC 4258 with SOFIA. ApJ869(1):61, DOI 10.3847/1538-4357/aaed2a, 1810.12883

Arav N, Borguet B, Chamberlain C, Edmonds D, Danforth C (2013) Quasar outflows and AGN feedback in the extreme UV: HST/COS observations of HE 0238-1904. MNRAS436(4):3286-3305, DOI 10. 1093/mnras/stt1812, 1305.2181

Arav N, Liu G, Xu X, Stidham J, Benn C, Chamberlain C (2018) Evidence that $50 \%$ of BALQSO Outflows Are Situated at Least 100 pc from the Central Source. ApJ857(1):60, DOI 10.3847/1538-4357/ aab494, 1805.01543

Arendt RG, Dwek E, Blair WP, Ghavamian P, Hwang U, Long KS, Petre R, Rho J, Winkler PF (2010) Spitzer Observations of Dust Destruction in the Puppis A Supernova Remnant. ApJ725(1):585-597, DOI 10.1088/0004-637X/725/1/585

Armillotta L, Fraternali F, Marinacci F (2016) Efficiency of gas cooling and accretion at the disc-corona interface. MNRAS462(4):4157-4170, DOI 10.1093/mnras/stw1930, 1608.06290

Armillotta L, Fraternali F, Werk JK, Prochaska JX, Marinacci F (2017) The survival of gas clouds in the circumgalactic medium of Milky Way-like galaxies. MNRAS470(1):114-125, DOI 10.1093/mnras/ stx1239, 1608.05416

Armillotta L, Krumholz MR, Di Teodoro EM, McClure-Griffiths NM (2019) The Life Cycle of the Central Molecular Zone. I: Inflow, Star Formation, and Winds. arXiv e-prints arXiv:1905.01309, 1905.01309

Arrigoni Battaia F, Hennawi JF, Prochaska JX, Oñorbe J, Farina EP, Cantalupo S, Lusso E (2019) QSO MUSEUM I: a sample of 61 extended Ly $\alpha$-emission nebulae surrounding $z \sim 3$ quasars. MNRAS482(3):3162-3205, DOI 10.1093/mnras/sty2827, 1808.10857

Asano RS, Takeuchi TT, Hirashita H, Inoue AK (2013) Dust formation history of galaxies: A critical role of metallicity for the dust mass growth by accreting materials in the interstellar medium. Earth, Planets, and Space 65(3):213-222, DOI 10.5047/eps.2012.04.014, 1206.0817

Asmus D, Gandhi P, Smette A, Hönig SF, Duschl WJ (2011) Mid-infrared properties of nearby lowluminosity AGN at high angular resolution. A\&A536:A36, DOI 10.1051/0004-6361/201116693, 1109.4873

Asmus D, Hönig SF, Gandhi P (2016) The Subarcsecond Mid-infrared View of Local Active Galactic Nuclei. III. Polar Dust Emission. ApJ822(2):109, DOI 10.3847/0004-637X/822/2/109, 1603.02710

Assef RJ, Eisenhardt PRM, Stern D, Tsai CW, Wu J, Wylezalek D, Blain AW, Bridge CR, Donoso E, Gonzales A, Griffith RL, Jarrett TH (2015) Half of the Most Luminous Quasars May Be Obscured: Investigating the Nature of WISE-Selected Hot Dust-Obscured Galaxies. ApJ804(1):27, DOI 10.1088/0004-637X/804/1/27, 1408. 1092

Baan WA, Haschick AD, Henkel C (1989) Molecular Outflows in Powerful OH Megamasers. ApJ346:680, DOI $10.1086 / 168050$

Bachiller R, Martin-Pintado J, Fuente A (1991) High-velocity SiO emission in the L1448 outflow. Evidence for dense shocked gas in the molecular bullets. A\&A243:L21

Bae HJ, Woo JH, Karouzos M, Gallo E, Flohic H, Shen Y, Yoon SJ (2017) The Limited Impact of Outflows: Integral-field Spectroscopy of 20 Local AGNs. ApJ837(1):91, DOI 10.3847/1538-4357/aa5f5c, 1702.01900

Baes M, Viaene S (2016) The nature of the UV halo around the spiral galaxy NGC 3628. A\&A587:A86, DOI 10.1051/0004-6361/201527812, 1601.06155

Bahcall JN, Ekers RD (1969) On the Possibility of Detecting Redshifted 21-CM Absorption Lines in the Spectra of Quasi-Stellar Sources. ApJ157:1055, DOI 10.1086/150135

Bally J (2016) Protostellar Outflows. ARA\&A54:491-528, DOI 10.1146/annurev-astro-081915-023341

Balmaverde B, Marconi A, Brusa M, Carniani S, Cresci G, Lusso E, Maiolino R, Mannucci F, Nagao T (2016) Is there any evidence that ionized outflows quench star formation in type 1 quasars at $\mathrm{z} \& \mathrm{lt} ; 1$ ? 
A\&A585:A148, DOI 10.1051/0004-6361/201526694, 1506.05984

Bambic CJ, Reynolds CS (2019) Efficient Production of Sound Waves by AGN Jets in the Intracluster Medium. arXiv e-prints arXiv:1906.03272, 1906.03272

Banda-Barragán WE, Parkin ER, Federrath C, Crocker RM, Bicknell GV (2016) Filament formation in wind-cloud interactions - I. Spherical clouds in uniform magnetic fields. MNRAS455(2):1309-1333, DOI 10.1093/mnras/stv2405, 1510.05356

Banda-Barragán WE, Federrath C, Crocker RM, Bicknell GV (2018) Filament formation in wind-cloud interactions- II. Clouds with turbulent density, velocity, and magnetic fields. MNRAS473(3):3454 3489, DOI 10.1093/mnras/stx2541, 1706.06607

Banda-Barragán WE, Zertuche FJ, Federrath C, García Del Valle J, Brüggen M, Wagner AY (2019) On the dynamics and survival of fractal clouds in galactic winds. MNRAS486(4):4526-4544, DOI 10.1093/ mnras/stz1040, 1901.06924

Banerji M, Alaghband-Zadeh S, Hewett PC, McMahon RG (2015) Heavily reddened type 1 quasars at z \&gt; 2 - I. Evidence for significant obscured black hole growth at the highest quasar luminosities. MNRAS447(4):3368-3389, DOI 10.1093/mnras/stu2649, 1501.00815

Barbosa FKB, Storchi-Bergmann T, McGregor P, Vale TB, Rogemar Riffel A (2014) Modelling the [Fe II] $\lambda 1.644 \mu \mathrm{m}$ outflow and comparison with $\mathrm{H}_{2}$ and $\mathrm{H}^{+}$kinematics in the inner $200 \mathrm{pc}$ of NGC 1068. MNRAS445(3):2353-2370, DOI 10.1093/mnras/stu1637, 1408.4750

Barcos-Muñoz L, Aalto S, Thompson TA, Sakamoto K, Martín S, Leroy AK, Privon GC, Evans AS, Kepley A (2018) Fast, Collimated Outflow in the Western Nucleus of Arp 220. ApJLett853(2):L28, DOI 10.3847/2041-8213/aaa28d, 1712.06381

Barger KA, Lehner N, Howk JC (2016) Down-the-barrel and Transverse Observations of the Large Magellanic Cloud: Evidence for a Symmetric Galactic Wind on the Near and Far Sides of the Galaxy. ApJ817(2):91, DOI 10.3847/0004-637X/817/2/91, 1512.00461

Barnes AT, Longmore SN, Battersby C, Bally J, Kruijssen JMD, Henshaw JD, Walker DL (2017) Star formation rates and efficiencies in the Galactic Centre. MNRAS469(2):2263-2285, DOI 10.1093/ mnras/stx941, 1704.03572

Baron D, Netzer H (2019a) Discovering AGN-driven winds through their infrared emission - I. General method and wind location. MNRAS482(3):3915-3932, DOI 10.1093/mnras/sty2935, 1810.06562

Baron D, Netzer H (2019b) Discovering AGN-driven winds through their infrared emission - II. Mass outflow rate and energetics. MNRAS486(3):4290-4303, DOI 10.1093/mnras/stz1070, 1903. 11076

Barvainis R (1987) Hot Dust and the Near-Infrared Bump in the Continuum Spectra of Quasars and Active Galactic Nuclei. ApJ320:537, DOI 10.1086/165571

Bauer M, Pietsch W, Trinchieri G, Breitschwerdt D, Ehle M, Read A (2007) High-resolution X-ray spectroscopy and imaging of the nuclear outflow of the starburst galaxy NGC 253. A\&A467(3):979-989, DOI 10.1051/0004-6361:20066340, astro-ph/0610302

Bautista MA, Dunn JP, Arav N, Korista KT, Moe M, Benn C (2010) Distance to Multiple Kinematic Components of Quasar Outflows: Very Large Telescope Observations of QSO 2359-1241 and SDSS J0318-0600. ApJ713(1):25-31, DOI 10.1088/0004-637X/713/1/25, 1003.0970

Behroozi PS, Wechsler RH, Conroy C (2013) The Average Star Formation Histories of Galaxies in Dark Matter Halos from $\mathrm{z}=$ 0-8. ApJ770(1):57, DOI 10.1088/0004-637X/770/1/57, 1207.6105

Beirão P, Armus L, Lehnert MD, Guillard P, Heckman T, Draine B, Hollenbach D, Walter F, Sheth K, Smith JD, Shopbell P, Boulanger F, Surace J, Hoopes C, Engelbracht C (2015) Spatially resolved Spitzer-IRS spectral maps of the superwind in M82. MNRAS451(3):2640-2655, DOI 10.1093/mnras/stv1101, 1505.04069

Belokurov V, Erkal D, Evans NW, Koposov SE, Deason AJ (2018) Co-formation of the disc and the stellar halo. MNRAS478(1):611-619, DOI 10.1093/mnras/sty982, 1802.03414

Belokurov V, Sanders JL, Fattahi A, Smith MC, Deason AJ, Evans NW, Grand RJJ (2019) The Biggest Splash. arXiv e-prints arXiv:1909.04679, 1909.04679

Bendo GJ, Beswick RJ, D’Cruze MJ, Dickinson C, Fuller GA, Muxlow TWB (2015) ALMA observations of $99 \mathrm{GHz}$ free-free and $\mathrm{H} 40 \alpha$ line emission from star formation in the centre of NGC 253. MNRAS450(1):L80-L84, DOI 10.1093/mnrasl/slv053, 1504.02142

Bertoldi F, McKee CF (1990) The Photoevaporation of Interstellar Clouds. II. Equilibrium Cometary Clouds. ApJ354:529, DOI 10.1086/168713

Bianchi S (2013) Vindicating single-T modified blackbody fits to Herschel SEDs. A\&A552:A89, DOI 10.1051/0004-6361/201220866, 1302.5699

Bieri R, Dubois Y, Silk J, Mamon GA (2015) Playing with Positive Feedback: External Pressuretriggering of a Star-forming Disk Galaxy. ApJLett812(2):L36, DOI 10.1088/2041-8205/812/2/L36, 
1503.05823

Bieri R, Dubois Y, Silk J, Mamon GA, Gaibler V (2016) External pressure-triggering of star formation in a disc galaxy: a template for positive feedback. MNRAS455(4):4166-4182, DOI 10.1093/mnras/ stv2551, 1507.00730

Biernacki P, Teyssier R (2018) The combined effect of AGN and supernovae feedback in launching massive molecular outflows in high-redshift galaxies. MNRAS475(4):5688-5703, DOI 10.1093/mnras/ sty216, 1712.02794

Bigiel F, Leroy A, Walter F, Brinks E, de Blok WJG, Madore B, Thornley MD (2008) The Star Formation Law in Nearby Galaxies on Sub-Kpc Scales. AJ136(6):2846-2871, DOI 10.1088/0004-6256/136/6/ $2846,0810.2541$

Binney J, Tremaine S (2008) Galactic Dynamics: Second Edition

Biscaro C, Cherchneff I (2014) Molecules and dust in Cassiopeia A. I. Synthesis in the supernova phase and processing by the reverse shock in the clumpy remnant. A\&A564:A25, DOI 10.1051/0004-6361/ 201322932, 1401. 5594

Biscaro C, Cherchneff I (2016) Molecules and dust in Cassiopeia A. II. Dust sputtering and diagnosis of supernova dust survival in remnants. A\&A589:A132, DOI 10.1051/0004-6361/201527769, 1511. 05487

Bischetti M, Maiolino R, Fiore SCF, Piconcelli E, Fluetsch A (2018) Widespread QSO-driven outflows in the early Universe. arXiv e-prints arXiv:1806.00786, 1806.00786

Bischetti M, Piconcelli E, Feruglio C, Fiore F, Carniani S, Brusa M, Cicone C, Vignali C, Bongiorno A, Cresci G, Mainieri V, Maiolino R, Marconi A, Nardini E, Zappacosta L (2019) The gentle monster PDS 456. Kiloparsec-scale molecular outflow and its implications for QSO feedback. A\&A628:A118, DOI 10.1051/0004-6361/201935524, 1903.10528

Bish HV, Werk JK, Prochaska JX, Rubin KHR, Zheng Y, O’Meara JM, Deason AJ (2019) Galactic Gas Flows from Halo to Disk: Tomography and Kinematics at the Milky Way's Disk-Halo Interface. ApJ882(2):76, DOI 10.3847/1538-4357/ab3414, 1907.09459

Bland-Hawthorn J, Cohen M (2003) The Large-Scale Bipolar Wind in the Galactic Center. ApJ582(1):246-256, DOI 10.1086/344573, astro-ph/0208553

Bland-Hawthorn J, Gerhard O (2016) The Galaxy in Context: Structural, Kinematic, and Integrated Properties. ARA\&A54:529-596, DOI 10.1146/annurev-astro-081915-023441, 1602.07702

Bland-Hawthorn J, Maloney PR, Sutherland RS, Madsen GJ (2013) Fossil Imprint of a Powerful Flare at the Galactic Center along the Magellanic Stream. ApJ778(1):58, DOI 10.1088/0004-637X/778/1/58, 1309.5455

Bland-Hawthorn J, Maloney P, Sutherland R, Groves B, Guglielmo M, Hao Li W, Curzons A, Cecil G, Fox A (2019) The large-scale ionization cones in the Galaxy. arXiv e-prints arXiv:1910.02225, 1910. 02225

Blandford RD, Payne DG (1982) Hydromagnetic flows from accretion disks and the production of radio jets. MNRAS199:883-903, DOI 10.1093/mnras/199.4.883

Blandford RD, Znajek RL (1977) Electromagnetic extraction of energy from Kerr black holes. MNRAS179:433-456, DOI 10.1093/mnras/179.3.433

de Blok WJG, Walter F, Ferguson AMN, Bernard EJ, van der Hulst JM, Neeleman M, Leroy AK, Ott J, Zschaechner LK, Zwaan MA, Yun MS, Langston G, Keating KM (2018) A High-resolution Mosaic of the Neutral Hydrogen in the M81 Triplet. ApJ865(1):26, DOI 10.3847/1538-4357/aad557, 1808. 02840

Bluck AFL, Mendel JT, Ellison SL, Patton DR, Simard L, Henriques BMB, Torrey P, Teimoorinia H, Moreno J, Starkenburg E (2016) The impact of galactic properties and environment on the quenching of central and satellite galaxies: a comparison between SDSS, Illustris and L-Galaxies. MNRAS462(3):2559-2586, DOI 10.1093/mnras/stw1665, 1607.03318

Bluck AFL, Maiolino R, Sanchez S, Ellison SL, Thorp MD, Piotrowska JM, Teimoorinia H, Bundy KA (2019) Are galactic star formation and quenching governed by local, global or environmental phenomena? arXiv e-prints arXiv:1911.08857, 1911.08857

Bocchio M, Bianchi S, Hunt LK, Schneider R (2016) Halo dust detection around NGC 891. A\&A586:A8, DOI 10.1051/0004-6361/201526950, 1509.07677

Boksenberg A, Carswell RF, Allen DA, Fosbury RAE, Penston MV, Sargent WLW (1977) The remarkable Seyfert galaxy Markarian 231. MNRAS178:451-466, DOI 10.1093/mnras/178.3.451

Bolatto AD, Warren SR, Leroy AK, Walter F, Veilleux S, Ostriker EC, Ott J, Zwaan M, Fisher DB, Weiss A, Rosolowsky E, Hodge J (2013a) Suppression of star formation in the galaxy NGC 253 by a starburst-driven molecular wind. Nature499(7459):450-453, DOI 10.1038/nature12351, 1307.6259 
Bolatto AD, Wolfire M, Leroy AK (2013b) The CO-to-H 2 Conversion Factor. ARA\&A51(1):207-268, DOI 10.1146/annurev-astro-082812-140944, 1301.3498

Bolatto AD, Armus L, Leroy AK, Veilleux S, Walter F, Mushotzky R (2018) How Do Cold Gas Outflows Shape Galaxies? In: Murphy E (ed) Science with a Next Generation Very Large Array, Astronomical Society of the Pacific Conference Series, vol 517, p 441

Bonato M, De Zotti G, Leisawitz D, Negrello M, Massardi M, Baronchelli I, Cai ZY, Bradford CM, Pope A, Murphy EJ, Armus L, Cooray A (2019) Origins Space Telescope: Predictions for far-IR spectroscopic surveys. PASA36:e017, DOI 10.1017/pasa.2019.8, 1903.00946

Bordoloi R, Lilly SJ, Knobel C, Bolzonella M, Kampczyk P, Carollo CM, Iovino A, Zucca E, Contini T, Kneib JP, Le Fevre O, Mainieri V, Renzini A, Scodeggio M, Zamorani G, Balestra I, Bardelli S, Bongiorno A, Caputi K, Cucciati O, de la Torre S, de Ravel L, Garilli B, Kovač K, Lamareille F, Le Borgne JF, Le Brun V, Maier C, Mignoli M, Pello R, Peng Y, Perez Montero E, Presotto V, Scarlata C, Silverman J, Tanaka M, Tasca L, Tresse L, Vergani D, Barnes L, Cappi A, Cimatti A, Coppa G, Diener C, Franzetti P, Koekemoer A, López-Sanjuan C, McCracken HJ, Moresco M, Nair P, Oesch P, Pozzetti L, Welikala N (2011) The Radial and Azimuthal Profiles of Mg II Absorption around 0.5 \&lt; z \&lt; 0.9 zCOSMOS Galaxies of Different Colors, Masses, and Environments. ApJ743(1):10, DOI 10.1088/0004-637X/743/1/10, 1106.0616

Bordoloi R, Lilly SJ, Hardmeier E, Contini T, Kneib JP, Le Fevre O, Mainieri V, Renzini A, Scodeggio M, Zamorani G, Bardelli S, Bolzonella M, Bongiorno A, Caputi K, Carollo CM, Cucciati O, de la Torre S, de Ravel L, Garilli B, Iovino A, Kampczyk P, Kovač K, Knobel C, Lamareille F, Le Borgne JF, Le Brun V, Maier C, Mignoli M, Oesch P, Pello R, Peng Y, Perez Montero E, Presotto V, Silverman J, Tanaka M, Tasca L, Tresse L, Vergani D, Zucca E, Cappi A, Cimatti A, Coppa G, Franzetti P, Koekemoer A, Moresco M, Nair P, Pozzetti L (2014a) The Dependence of Galactic Outflows on the Properties and Orientation of zCOSMOS Galaxies at $z \sim 1$. ApJ794(2):130, DOI 10.1088/0004-637X/794/2/130, 1307.6553

Bordoloi R, Tumlinson J, Werk JK, Oppenheimer BD, Peeples MS, Prochaska JX, Tripp TM, Katz N, Davé R, Fox AJ, Thom C, Ford AB, Weinberg DH, Burchett JN, Kollmeier JA (2014b) The COS-Dwarfs Survey: The Carbon Reservoir around Sub-L* Galaxies. ApJ796(2):136, DOI 10.1088/0004-637X/ 796/2/136, 1406.0509

Bordoloi R, Rigby JR, Tumlinson J, Bayliss MB, Sharon K, Gladders MG, Wuyts E (2016) Spatially resolved galactic wind in lensed galaxy RCSGA 032727-132609. MNRAS458(2):1891-1908, DOI $10.1093 /$ mnras/stw449, 1602.07700

Bordoloi R, Fox AJ, Lockman FJ, Wakker BP, Jenkins EB, Savage BD, Hernandez S, Tumlinson J, BlandHawthorn J, Kim TS (2017a) Mapping the Nuclear Outflow of the Milky Way: Studying the Kinematics and Spatial Extent of the Northern Fermi Bubble. ApJ834(2):191, DOI 10.3847/1538-4357/ 834/2/191, 1612.01578

Bordoloi R, Wagner AY, Heckman TM, Norman CA (2017b) The Formation and Physical Origin of Highly Ionized Cooling Gas. ApJ848(2):122, DOI 10.3847/1538-4357/aa8e9c, 1605.07187

Boreiko RT, Betz AL (1996) The 12C/ 13C Isotopic Ratio in Photodissociated Gas in M42. ApJLett467:L113, DOI 10.1086/310204, astro-ph/9606143

Borisova E, Cantalupo S, Lilly SJ, Marino RA, Gallego SG, Bacon R, Blaizot J, Bouché N, Brinchmann J, Carollo CM, Caruana J, Finley H, Herenz EC, Richard J, Schaye J, Straka LA, Turner ML, Urrutia T, Verhamme A, Wisotzki L (2016) Ubiquitous Giant Ly $\alpha$ Nebulae around the Brightest Quasars at $z \sim 3.5$ Revealed with MUSE. ApJ831(1):39, DOI 10.3847/0004-637X/831/1/39, 1605.01422

Bouché N, Hohensee W, Vargas R, Kacprzak GG, Martin CL, Cooke J, Churchill CW (2012) Physical properties of galactic winds using background quasars. MNRAS426(2):801-815, DOI 10.1111/j. 1365-2966.2012.21114.x, 1110.5877

Bourne MA, Sijacki D (2017) AGN jet feedback on a moving mesh: cocoon inflation, gas flows and turbulence. MNRAS472(4):4707-4735, DOI 10.1093/mnras/stx2269, 1705.07900

Bouwman J, Meeus G, de Koter A, Hony S, Dominik C, Waters LBFM (2001) Processing of silicate dust grains in Herbig Ae/Be systems. A\&A375:950-962, DOI 10.1051/0004-6361:20010878

Bowen DV, Jenkins EB, Tripp TM, Sembach KR, Savage BD, Moos HW, Oegerle WR, Friedman SD, Gry C, Kruk JW, Murphy E, Sankrit R, Shull JM, Sonneborn G, York DG (2008) The Far Ultraviolet Spectroscopic Explorer Survey of O VI Absorption in the Disk of the Milky Way. ApJSupp.176(1):59-163, DOI 10.1086/524773, 0711.0005

Bower RG, Schaye J, Frenk CS, Theuns T, Schaller M, Crain RA, McAlpine S (2017) The dark nemesis of galaxy formation: why hot haloes trigger black hole growth and bring star formation to an end. MNRAS465(1):32-44, DOI 10.1093/mnras/stw2735, 1607.07445 
Bregman JN (1980) The galactic fountain of high-velocity clouds. ApJ236:577-591, DOI 10.1086/157776

Bregman JN, Miller ED, Seitzer P, Cowley CR, Miller MJ (2013) Outflow versus Infall in Spiral Galaxies: Metal Absorption in the Halo of NGC 891. ApJ766(1):57, DOI 10.1088/0004-637X/766/1/57, 1304. 0795

Brennan R, Choi E, Somerville RS, Hirschmann M, Naab T, Ostriker JP (2018) Momentum-driven Winds from Radiatively Efficient Black Hole Accretion and Their Impact on Galaxies. ApJ860(1):14, DOI 10.3847/1538-4357/aac2c4, 1805.00946

Brüggen M, Scannapieco E (2016) The Launching of Cold Clouds by Galaxy Outflows. II. The Role of Thermal Conduction. ApJ822(1):31, DOI 10.3847/0004-637X/822/1/31, 1602.01843

Brusa M, Cresci G, Daddi E, Paladino R, Perna M, Bongiorno A, Lusso E, Sargent MT, Casasola V, Feruglio C, Fraternali F, Georgiev I, Mainieri V, Carniani S, Comastri A, Duras F, Fiore F, Mannucci F, Marconi A, Piconcelli E, Zamorani G, Gilli R, La Franca F, Lanzuisi G, Lutz D, Santini P, Scoville NZ, Vignali C, Vito F, Rabien S, Busoni L, Bonaglia M (2018) Molecular outflow and feedback in the obscured quasar XID2028 revealed by ALMA. A\&A612:A29, DOI 10.1051/0004-6361/201731641, 1712.04505

Burtscher L, Meisenheimer K, Tristram KRW, Jaffe W, Hönig SF, Davies RI, Kishimoto M, Pott JU, Röttgering H, Schartmann M, Weigelt G, Wolf S (2013) A diversity of dusty AGN tori. Data release for the VLTI/MIDI AGN Large Program and first results for 23 galaxies. A\&A558:A149, DOI 10. 1051/0004-6361/201321890, 1307.2068

Bustard C, Zweibel EG, D’Onghia E, Gallagher I J S, Farber R (2019) Cosmic Ray Driven Outflows from the Large Magellanic Cloud: Contributions to the LMC Filament. arXiv e-prints arXiv:1911.02021, 1911.02021

Bykov AM, Ellison DC, Marcowith A, Osipov SM (2018) Cosmic Ray Production in Supernovae. SSR214(1):41, DOI 10.1007/s11214-018-0479-4, 1801.08890

Cai Z, Cantalupo S, Prochaska JX, Arrigoni Battaia F, Burchett J, Li Q, Chisholm J, Bundy K, Hennawi JF (2019) Evolution of the Cool Gas in the Circumgalactic Medium (CGM) of Massive Halos - A Keck Cosmic Web Imager (KCWI) Survey of Ly $\alpha$ Emission around QSOs at $z \approx 2$. arXiv e-prints arXiv: $1909.11098,1909.11098$

Cano-Díaz M, Maiolino R, Marconi A, Netzer H, Shemmer O, Cresci G (2012) Observational evidence of quasar feedback quenching star formation at high redshift. A\&A537:L8, DOI 10.1051/0004-6361/ 201118358, 1112.3071

Cantalupo S, Porciani C, Lilly SJ (2008) Mapping Neutral Hydrogen during Reionization with the Ly $\alpha$ Emission from Quasar Ionization Fronts. ApJ672(1):48-58, DOI 10.1086/523298, 0709.0654

Cardelli JA, Clayton GC, Mathis JS (1989) The Relationship between Infrared, Optical, and Ultraviolet Extinction. ApJ345:245, DOI 10.1086/167900

Carniani S, Marconi A, Maiolino R, Balmaverde B, Brusa M, Cano-Díaz M, Cicone C, Comastri A, Cresci G, Fiore F, Feruglio C, La Franca F, Mainieri V, Mannucci F, Nagao T, Netzer H, Piconcelli E, Risaliti G, Schneider R, Shemmer O (2015) Ionised outflows in $z \sim 2.4$ quasar host galaxies. A\&A580:A102, DOI 10.1051/0004-6361/201526557, 1506.03096

Carniani S, Marconi A, Maiolino R, Balmaverde B, Brusa M, Cano-Díaz M, Cicone C, Comastri A, Cresci G, Fiore F, Feruglio C, La Franca F, Mainieri V, Mannucci F, Nagao T, Netzer H, Piconcelli E, Risaliti G, Schneider R, Shemmer O (2016) Fast outflows and star formation quenching in quasar host galaxies. A\&A591:A28, DOI 10.1051/0004-6361/201528037, 1604.04290

Carniani S, Marconi A, Maiolino R, Feruglio C, Brusa M, Cresci G, Cano-Díaz M, Cicone C, Balmaverde B, Fiore F, Ferrara A, Gallerani S, La Franca F, Mainieri V, Mannucci F, Netzer H, Piconcelli E, Sani E, Schneider R, Shemmer O, Testi L (2017) AGN feedback on molecular gas reservoirs in quasars at z 2.4. A\&A605:A105, DOI 10.1051/0004-6361/201730672, 1706.08987

Carroll TJ, Goldsmith PF (1981) Infrared pumping and rotational excitation of molecules in interstellar clouds. ApJ245:891-897, DOI 10.1086/158865

Cazzoli S, Arribas S, Maiolino R, Colina L (2016) Neutral gas outflows in nearby [U]LIRGs via optical $\mathrm{NaD}$ feature. A\&A590:A125, DOI 10.1051/0004-6361/201526788, 1602.08505

Cecil G, Bland J, Tully RB (1990) Imaging Spectrophotometry of Ionized Gas in NGC 1068. I. Kinematics of the Narrow-Line Region. ApJ355:70, DOI 10.1086/168742

Cecil G, Dopita MA, Groves B, Wilson AS, Ferruit P, Pécontal E, Binette L (2002) Spatial Resolution of High-Velocity Filaments in the Narrow-Line Region of NGC 1068: Associated Absorbers Caught in Emission? ApJ568(2):627-638, DOI 10.1086/338950, astro-ph/0112256

Cernicharo J, Goicoechea JR, Daniel F, Lerate MR, Barlow MJ, Swinyard BM, van Dishoeck EF, Lim TL, Viti S, Yates J (2006) The Water Vapor Abundance in Orion KL Outflows. ApJLett649(1):L33-L36, 
DOI 10.1086/508259, astro-ph/0608336

Chan TK, Kereš D, Hopkins PF, Quataert E, Su KY, Hayward CC, Faucher-Giguère CA (2019) Cosmic ray feedback in the FIRE simulations: constraining cosmic ray propagation with $\mathrm{GeV} \gamma$-ray emission. MNRAS488(3):3716-3744, DOI 10.1093/mnras/stz1895, 1812.10496

Chastenet J, Sandstrom K, Chiang ID, Leroy AK, Utomo D, Bot C, Gordon KD, Draine BT, Fukui Y, Onishi T, Tsuge K (2019) The Polycyclic Aromatic Hydrocarbon Mass Fraction on a 10 pc Scale in the Magellanic Clouds. ApJ876(1):62, DOI 10.3847/1538-4357/ab16cf, 1904.02705

Chen YM, Tremonti CA, Heckman TM, Kauffmann G, Weiner BJ, Brinchmann J, Wang J (2010) Absorption-line Probes of the Prevalence and Properties of Outflows in Present-day Star-forming Galaxies. AJ140(2):445-461, DOI 10.1088/0004-6256/140/2/445, 1003. 5425

Chevalier RA, Clegg AW (1985) Wind from a starburst galaxy nucleus. Nature317(6032):44-45, DOI $10.1038 / 317044 \mathrm{a} 0$

Chisholm J, Tremonti CA, Leitherer C, Chen Y, Wofford A, Lundgren B (2015) Scaling Relations Between Warm Galactic Outflows and Their Host Galaxies. ApJ811(2):149, DOI 10.1088/0004-637X/811/2/ $149,1412.2139$

Chisholm J, Tremonti CA, Leitherer C, Chen Y, Wofford A (2016a) Shining a light on galactic outflows: photoionized outflows. MNRAS457(3):3133-3161, DOI 10.1093/mnras/stw178, 1601.05090

Chisholm J, Tremonti Christy A, Leitherer C, Chen Y (2016b) A robust measurement of the mass outflow rate of the galactic outflow from NGC 6090. MNRAS463(1):541-556, DOI 10.1093/mnras/stw1951, 1605.05769

Chisholm J, Tremonti CA, Leitherer C, Chen Y (2017) The mass and momentum outflow rates of photoionized galactic outflows. MNRAS469(4):4831-4849, DOI 10.1093/mnras/stx1164, 1702.07351

Chisholm J, Tremonti C, Leitherer C (2018) Metal-enriched galactic outflows shape the mass-metallicity relationship. MNRAS481(2):1690-1706, DOI 10.1093/mnras/sty2380, 1808.10453

Choi E, Somerville RS, Ostriker JP, Naab T, Hirschmann M (2018) The Role of Black Hole Feedback on Size and Structural Evolution in Massive Galaxies. ApJ866(2):91, DOI 10.3847/1538-4357/aae076, 1809.02143

Chu YH, Mac Low MM, Garcia-Segura G, Wakker B, Kennicutt J Robert C (1993) Hidden Supernova Remnants in the Large Magellanic Cloud H II Complex N44. ApJ414:213, DOI 10.1086/173069

Cicone C, Feruglio C, Maiolino R, Fiore F, Piconcelli E, Menci N, Aussel H, Sturm E (2012) The physics and the structure of the quasar-driven outflow in Mrk 231. A\&A543:A99, DOI 10.1051/0004-6361/ 201218793, 1204.5881

Cicone C, Maiolino R, Sturm E, Graciá-Carpio J, Feruglio C, Neri R, Aalto S, Davies R, Fiore F, Fischer J, García-Burillo S, González-Alfonso E, Hailey-Dunsheath S, Piconcelli E, Veilleux S (2014) Massive molecular outflows and evidence for AGN feedback from CO observations. A\&A562:A21, DOI $10.1051 / 0004-6361 / 201322464,1311.2595$

Cicone C, Maiolino R, Gallerani S, Neri R, Ferrara A, Sturm E, Fiore F, Piconcelli E, Feruglio C (2015) Very extended cold gas, star formation and outflows in the halo of a bright quasar at $\mathrm{z} \& \mathrm{gt} ; 6$. A\&A574:A14, DOI 10.1051/0004-6361/201424980, 1409.4418

Cicone C, Brusa M, Ramos Almeida C, Cresci G, Husemann B, Mainieri V (2018a) The largely unconstrained multiphase nature of outflows in AGN host galaxies. Nature Astronomy 2:176-178, DOI 10.1038/s41550-018-0406-3, 1802.10308

Cicone C, Severgnini P, Papadopoulos PP, Maiolino R, Feruglio C, Treister E, Privon GC, Zhang Zy, Della Ceca R, Fiore F, Schawinski K, Wagg J (2018b) ALMA [C I $]^{3} \mathrm{P}_{1^{-}}{ }^{3} \mathrm{P}_{0}$ Observations of NGC 6240: A Puzzling Molecular Outflow, and the Role of Outflows in the Global $\alpha$ co Factor of (U)LIRGs. ApJ863(2):143, DOI 10.3847/1538-4357/aad32a, 1807.06015

Cicone C, Maiolino R, Aalto S, Muller S, Feruglio C (2019) Enhanced UV radiation and dense clumps in Mrk231's molecular outflow. arXiv e-prints arXiv:1911.11243, 1911.11243

Cimatti A, di Serego Alighieri S, Vernet J, Cohen MH, Fosbury RAE (1998) The UV Radiation from Z approximately 2.5 Radio Galaxies: Keck Spectropolarimetry of 4C 23.56 and 4C 00.54 ApJLett499(1):L21-L25, DOI 10.1086/311354, astro-ph/9803311

Clark PC, Glover SCO, Klessen RS, Bonnell IA (2012) How long does it take to form a molecular cloud? MNRAS424(4):2599-2613, DOI 10.1111/j.1365-2966.2012.21259.x, 1204.5570

Coatman L, Hewett PC, Banerji M, Richards GT, Hennawi JF, Prochaska JX (2019) Kinematics of C IV and [O III] emission in luminous high-redshift quasars. MNRAS486(4):5335-5348, DOI 10.1093/ mnras/stz1167, 1904.13348

Coil AL, Weiner BJ, Holz DE, Cooper MC, Yan R, Aird J (2011) Outflowing Galactic Winds in Poststarburst and Active Galactic Nucleus Host Galaxies at 0.2 \&lt; z \&lt; 0.8. ApJ743(1):46, DOI 10. 
1088/0004-637X/743/1/46, 1104.0681

Combes F, García-Burillo S, Casasola V, Hunt L, Krips M, Baker AJ, Boone F, Eckart A, Marquez I, Neri R, Schinnerer E, Tacconi LJ (2014) ALMA observations of feeding and feedback in nearby Seyfert galaxies: an AGN-driven outflow in NGC 1433 (Corrigendum). A\&A564:C1, DOI 10.1051/ 0004-6361/201322288e

Concas A, Popesso P, Brusa M, Mainieri V, Thomas D (2019) Two-face(s): ionized and neutral gas winds in the local Universe. A\&A622:A188, DOI 10.1051/0004-6361/201732152

Conroy C, van Dokkum PG, Kravtsov A (2015) Preventing Star Formation in Early-Type Galaxies with Late-Time Stellar Heating. ApJ803(2):77, DOI 10.1088/0004-637X/803/2/77, 1406. 3026

Contursi A, Poglitsch A, Graciá Carpio J, Veilleux S, Sturm E, Fischer J, Verma A, Hailey-Dunsheath S, Lutz D, Davies R, González-Alfonso E, Sternberg A, Genzel R, Tacconi L (2013) Spectroscopic FIR mapping of the disk and galactic wind of M 82 with Herschel-PACS. A\&A549:A118, DOI 10.1051/0004-6361/201219214, 1210.3496

Cooper JL, Bicknell GV, Sutherland RS, Bland-Hawthorn J (2009) Starburst-Driven Galactic Winds: Filament Formation and Emission Processes. ApJ703(1):330-347, DOI 10.1088/0004-637X/703/1/ $330,0907.4004$

Costa T, Sijacki D, Haehnelt MG (2014) Feedback from active galactic nuclei: energy- versus momentumdriving. MNRAS444(3):2355-2376, DOI 10.1093/mnras/stu1632, 1406.2691

Costa T, Sijacki D, Haehnelt MG (2015) Fast cold gas in hot AGN outflows. MNRAS448:L30-L34, DOI $10.1093 / \mathrm{mnrasl} / \mathrm{slu} 193,1411.0678$

Costa T, Rosdahl J, Sijacki D, Haehnelt MG (2018a) Driving gas shells with radiation pressure on dust in radiation-hydrodynamic simulations. MNRAS473(3):4197-4219, DOI 10.1093/mnras/stx2598, 1703.05766

Costa T, Rosdahl J, Sijacki D, Haehnelt MG (2018b) Quenching star formation with quasar outflows launched by trapped IR radiation. MNRAS479(2):2079-2111, DOI 10.1093/mnras/sty1514, 1709. 08638

Costagliola F, Aalto S (2010) Vibrationally excited $\mathrm{HC}_{3} \mathrm{~N}$ in NGC 4418. A\&A515:A71, DOI 10.1051/ 0004-6361/200913370, 1003.3141

Costagliola F, Aalto S, Sakamoto K, Martín S, Beswick R, Muller S, Klöckner HR (2013) High-resolution $\mathrm{mm}$ and $\mathrm{cm}$ study of the obscured LIRG NGC 4418 . A compact obscured nucleus fed by in-falling gas? A\&A556:A66, DOI 10.1051/0004-6361/201220634, 1306.2211

Costagliola F, Herrero-Illana R, Lohfink A, Pérez-Torres M, Aalto S, Muller S, Alberdi A (2016) Radio continuum and $\mathrm{X}$-ray emission from the most extreme far-IR-excess galaxy ${ }_{i} \mathrm{ASTROBJ}_{i} \mathrm{NGC}_{\mathrm{A}}$ $1377_{i} /$ ASTROBJ $_{i}$. An extremely obscured AGN revealed. A\&A594:A114, DOI 10.1051/0004-6361/ 201628997, 1607.04068

Crawford MK, Genzel R, Townes CH, Watson DM (1985) Far-infrared spectroscopy of galaxies : the 158 micron C+ line and the energy balance of molecular clouds. ApJ291:755-771, DOI 10.1086/163113

Crenshaw DM, Kraemer SB (2000) Resolved Spectroscopy of the Narrow-Line Region in NGC 1068: Kinematics of the Ionized Gas. ApJLett532(2):L101-L104, DOI 10.1086/312581, astro-ph/ 0002438

Cresci G, Mainieri V, Brusa M, Marconi A, Perna M, Mannucci F, Piconcelli E, Maiolino R, Feruglio C, Fiore F, Bongiorno A, Lanzuisi G, Merloni A, Schramm M, Silverman JD, Civano F (2015) Blowin' in the Wind: Both "Negative" and "Positive" Feedback in an Obscured High-z Quasar. ApJ799(1):82, DOI 10.1088/0004-637X/799/1/82, 1411.4208

Crinklaw G, Federman SR, Joseph CL (1994) The Depletion of Calcium in the Interstellar Medium. ApJ424:748, DOI 10.1086/173927

Crocker AF, Pellegrini E, Smith JDT, Draine BT, Wilson CD, Wolfire M, Armus L, Brinks E, Dale DA, Groves B, Herrera-Camus R, Hunt LK, Kennicutt RC, Murphy EJ, Sandstrom K, Schinnerer E, Rigopoulou D, Rosolowsky E, van der Werf P (2019) [C I](1-0) and [C I](2-1) in Resolved Local Galaxies. ApJ887(1):105, DOI 10.3847/1538-4357/ab4196

Crocker RM, Aharonian F (2011) Fermi Bubbles: Giant, Multibillion-Year-Old Reservoirs of Galactic Center Cosmic Rays. PRL106(10):101102, DOI 10.1103/PhysRevLett.106.101102, 1008.2658

Crocker RM, Bicknell GV, Taylor AM, Carretti E (2015) A Unified Model of the Fermi Bubbles, Microwave Haze, and Polarized Radio Lobes: Reverse Shocks in the Galactic Center's Giant Outflows. ApJ808(2):107, DOI 10.1088/0004-637X/808/2/107, 1412.7510

Crocker RM, Krumholz MR, Thompson TA, Clutterbuck J (2018) The maximum flux of star-forming galaxies. MNRAS478(1):81-94, DOI 10.1093/mnras/sty989, 1802.03117

Crockett RM, Shabala SS, Kaviraj S, Antonuccio-Delogu V, Silk J, Mutchler M, O’Connell RW, Rejkuba 
M, Whitmore BC, Windhorst RA (2012) Triggered star formation in the inner filament of Centaurus A. MNRAS421(2):1603-1623, DOI 10.1111/j.1365-2966.2012.20418.x, 1201.3369

Croft S, van Breugel W, de Vries W, Dopita M, Martin C, Morganti R, Neff S, Oosterloo T, Schiminovich D, Stanford SA, van Gorkom J (2006) Minkowski's Object: A Starburst Triggered by a Radio Jet, Revisited. ApJ647(2):1040-1055, DOI 10.1086/505526, astro-ph/0604557

Curran SJ, Allison JR, Whiting MT, Sadler EM, Combes F, Pracy MB, Bignell C, Athreya R (2016) A search for $\mathrm{H}$ I and $\mathrm{OH}$ absorption in $\mathrm{z} \gtrsim 3$ CO emitters. MNRAS457(4):3666-3677, DOI 10.1093/ mnras/stw089, 1601.01971

Curran SJ, Hunstead RW, Johnston HM, Whiting MT, Sadler EM, Allison JR, Athreya R (2019) Ionization of the atomic gas in redshifted radio sources. MNRAS484(1):1182-1191, DOI 10.1093/mnras/stz038, 1901.00887

Das V, Crenshaw DM, Kraemer SB, Deo RP (2006) Kinematics of the Narrow-Line Region in the Seyfert 2 Galaxy NGC 1068: Dynamical Effects of the Radio Jet. AJ132(2):620-632, DOI 10.1086/504899, astro-ph/0603803

Dasyra KM, Combes F (2012) Cold and warm molecular gas in the outflow of 4C 12.50. A\&A541:L7, DOI 10.1051/0004-6361/201219229, 1203.3452

Dasyra KM, Bostrom AC, Combes F, Vlahakis N (2015) A Radio Jet Drives a Molecular and Atomic Gas Outflow in Multiple Regions within One Square Kiloparsec of the Nucleus of the nearby Galaxy IC5063. ApJ815(1):34, DOI 10.1088/0004-637X/815/1/34, 1503.05484

Dasyra KM, Combes F, Oosterloo T, Oonk JBR, Morganti R, Salomé P, Vlahakis N (2016) ALMA reveals optically thin, highly excited CO gas in the jet-driven winds of the galaxy IC 5063. A\&A595:L7, DOI 10.1051/0004-6361/201629689, 1609.03421

Davé R, Finlator K, Oppenheimer BD (2011) Galaxy evolution in cosmological simulations with outflows - II. Metallicities and gas fractions. MNRAS416(2):1354-1376, DOI 10.1111/j.1365-2966. 2011.19132.x, 1104.3156

Davé R, Anglés-Alcázar D, Narayanan D, Li Q, Rafieferantsoa MH, Appleby S (2019) SIMBA: Cosmological simulations with black hole growth and feedback. MNRAS486(2):2827-2849, DOI $10.1093 / \mathrm{mnras} / \mathrm{stz} 937,1901.10203$

Davies JJ, Crain RA, Oppenheimer BD, Schaye J (2019) The quenching and morphological evolution of central galaxies is facilitated by the feedback-driven expulsion of circumgalactic gas. arXiv e-prints arXiv: $1908.11380,1908.11380$

Davies R (2008) Adaptive optics: Observations and prospects for studies of active Galactic Nuclei. New Astron. Rev.52(6):307-322, DOI 10.1016/j.newar.2008.06.002, 0806.0468

Davies RL, Kewley LJ, Ho IT, Dopita MA (2014) Starburst-AGN mixing - II. Optically selected active galaxies. MNRAS444(4):3961-3974, DOI 10.1093/mnras/stu1740, 1408. 5888

Davies RL, Groves B, Kewley LJ, Dopita MA, Hampton EJ, Shastri P, Scharwächter J, Sutherland R, Kharb P, Bhatt H, Jin C, Banfield J, Zaw I, James B, Juneau S, Srivastava S (2016) Dissecting galaxies: spatial and spectral separation of emission excited by star formation and AGN activity. MNRAS462(2):1616-1629, DOI 10.1093/mnras/stw1754, 1607.05731

Davis TA, Krajnović D, McDermid RM, Bureau M, Sarzi M, Nyland K, Alatalo K, Bayet E, Blitz L, Bois M, Bournaud F, Cappellari M, Crocker A, Davies RL, de Zeeuw PT, Duc PA, Emsellem E, Khochfar S, Kuntschner H, Lablanche PY, Morganti R, Naab T, Oosterloo T, Scott N, Serra P, Weijmans AM, Young LM (2012) Gemini GMOS and WHT SAURON integral-field spectrograph observations of the AGN-driven outflow in NGC 1266. MNRAS426(2):1574-1590, DOI 10.1111/j.1365-2966.2012. 21770.x, 1207.5799

De Cia A (2018) Metals and dust in the neutral ISM: the Galaxy, Magellanic Clouds, and damped Lyman- $\alpha$ absorbers. A\&A613:L2, DOI 10.1051/0004-6361/201833034, 1805.05365

De Cia A, Ledoux C, Mattsson L, Petitjean P, Srianand R, Gavignaud I, Jenkins EB (2016) Dust-depletion sequences in damped Lyman- $\alpha$ absorbers. A unified picture from low-metallicity systems to the Galaxy. A\&A596:A97, DOI 10.1051/0004-6361/201527895, 1608.08621

De Young DS (1981) Emission line regions and stellar associations in extended extragalactic radio sources. Nature293(5827):43-44, DOI 10.1038/293043a0

Debuhr J, Quataert E, Ma CP (2012) Galaxy-scale outflows driven by active galactic nuclei. MNRAS420(3):2221-2231, DOI 10.1111/j.1365-2966.2011.20187.x, 1107.5579

Decataldo D, Ferrara A, Pallottini A, Gallerani S, Vallini L (2017) Molecular clumps photoevaporation in ionized regions. MNRAS471(4):4476-4487, DOI 10.1093/mnras/stx1879, 1707.08574

Decataldo D, Pallottini A, Ferrara A, Vallini L, Gallerani S (2019) Photoevaporation of Jeans-unstable molecular clumps. MNRAS487(3):3377-3391, DOI 10.1093/mnras/stz1527, 1905.13230 
Dessauges-Zavadsky M, Prochaska JX, D’Odorico S, Calura F, Matteucci F (2006) A new comprehensive set of elemental abundances in DLAs. II. Data analysis and chemical variation studies. A\&A445(1):93-113, DOI 10.1051/0004-6361:20053200, astro-ph/0511031

Dessauges-Zavadsky M, D’Odorico S, Schaerer D, Modigliani A, Tapken C, Vernet J (2010) Rest-frame ultraviolet spectrum of the gravitationally lensed galaxy "the 8 o'clock arc": stellar and interstellar medium properties. A\&A510:A26, DOI 10.1051/0004-6361/200913337, 0912 .4384

Devine D, Bally J (1999) H $\alpha$ Emission 11 Kiloparsecs above M82. ApJ510(1):197-204, DOI 10.1086/ 306582

Di Matteo T, Springel V, Hernquist L (2005) Energy input from quasars regulates the growth and activity of black holes and their host galaxies. Nature433(7026):604-607, DOI 10.1038/nature03335, astro-ph/0502199

di Serego Alighieri S, Cimatti A, Fosbury RAE, Hes R (1997) Anisotropic [OIII] emission in radio loud AGN. A\&A328:510-516, astro-ph/9708205

Di Teodoro EM, Fraternali F (2015) ${ }^{3 D}$ BAROLO: a new 3D algorithm to derive rotation curves of galaxies. MNRAS451(3):3021-3033, DOI 10.1093/mnras/stv1213, 1505.07834

Di Teodoro EM, McClure-Griffiths NM, Lockman FJ, Denbo SR, Endsley R, Ford HA, Harrington K (2018) Blowing in the Milky Way Wind: Neutral Hydrogen Clouds Tracing the Galactic Nuclear Outflow. ApJ855(1):33, DOI 10.3847/1538-4357/aaad6a, 1802.02152

Di Teodoro EM, McClure-Griffiths NM, De Breuck C, Armillotta L, Pingel NM, Jameson KE, Dickey JM, Rubio M, Stanimirović S, Staveley-Smith L (2019) Molecular Gas in the Outflow of the Small Magellanic Cloud. ApJLett885(2):L32, DOI 10.3847/2041-8213/ab4fe9

Diamond-Stanic AM, Moustakas J, Tremonti CA, Coil AL, Hickox RC, Robaina AR, Rudnick GH, Sell PH (2012) High-velocity Outflows without AGN Feedback: Eddington-limited Star Formation in Compact Massive Galaxies. ApJLett755(2):L26, DOI 10.1088/2041-8205/755/2/L26, 1205.2368

Díaz-Santos T, Assef RJ, Blain AW, Tsai CW, Aravena M, Eisenhardt P, Wu J, Stern D, Bridge C (2016) The Strikingly Uniform, Highly Turbulent Interstellar Medium of the Most Luminous Galaxy in the Universe. ApJLett816(1):L6, DOI 10.3847/2041-8205/816/1/L6, 1511.04079

Diesing R, Caprioli D (2018) Effect of Cosmic Rays on the Evolution and Momentum Deposition of Supernova Remnants. PRL121(9):091101, DOI 10.1103/PhysRevLett.121.091101, 1804.09731

Dijkstra M (2017) Saas-Fee Lecture Notes: Physics of Lyman Alpha Radiative Transfer. arXiv e-prints arXiv:1704.03416, 1704.03416

Dobler G, Finkbeiner DP (2008) Extended Anomalous Foreground Emission in the WMAP Three-Year Data. ApJ680(2):1222-1234, DOI 10.1086/587862, 0712 . 1038

Dobler G, Finkbeiner DP, Cholis I, Slatyer T, Weiner N (2010) The Fermi Haze: A Gamma-ray Counterpart to the Microwave Haze. ApJ717(2):825-842, DOI 10.1088/0004-637X/717/2/825, 0910.4583

Domgorgen H, Mathis JS (1994) The Ionization of the Diffuse Ionized Gas. ApJ428:647, DOI 10.1086/ 174275

Dopita MA, Seitenzahl IR, Sutherland RS, Vogt FPA, Winkler PF, Blair WP (2016) Forbidden Iron Lines and Dust Destruction in Supernova Remnant Shocks: The Case of N49 in the Large Magellanic Cloud. ApJ826(2):150, DOI 10.3847/0004-637X/826/2/150, 1605.02385

Doyon R, Wright GS, Joseph RD (1994) A Near-Infrared Spectroscopic Study of the Luminous Merger NGC 3256. II. Evidence for Fluorescent Molecular Hydrogen Emission. ApJ421:115, DOI 10.1086/ 173630

Draine BT (2003) Interstellar Dust Grains. ARA\&A41:241-289, DOI 10.1146/annurev.astro.41.011802. 094840, astro-ph/0304489

Draine BT (2009) Interstellar Dust Models and Evolutionary Implications. In: Henning T, Grün E, Steinacker J (eds) Cosmic Dust - Near and Far, Astronomical Society of the Pacific Conference Series, vol 414, p 453, 0903.1658

Draine BT (2011) Physics of the Interstellar and Intergalactic Medium

Draine BT, Allaf-Akbari K (2006) X-Ray Scattering by Nonspherical Grains. I. Oblate Spheroids. ApJ652(2):1318-1330, DOI 10.1086/508133, astro-ph/0608037

Draine BT, Fraisse AA (2009) Polarized Far-Infrared and Submillimeter Emission from Interstellar Dust. ApJ696(1):1-11, DOI 10.1088/0004-637X/696/1/1, 0809.2094

Draine BT, Li A (2007) Infrared Emission from Interstellar Dust. IV. The Silicate-Graphite-PAH Model in the Post-Spitzer Era. ApJ657(2):810-837, DOI 10.1086/511055, astro-ph/0608003

Draine BT, Salpeter EE (1979a) Destruction mechanisms for interstellar dust. ApJ231:438-455, DOI $10.1086 / 157206$

Draine BT, Salpeter EE (1979b) On the physics of dust grains in hot gas. ApJ231:77-94, DOI 10.1086/ 
157165

Draine BT, Dale DA, Bendo G, Gordon KD, Smith JDT, Armus L, Engelbracht CW, Helou G, Kennicutt J R C, Li A, Roussel H, Walter F, Calzetti D, Moustakas J, Murphy EJ, Rieke GH, Bot C, Hollenbach DJ, Sheth K, Teplitz HI (2007) Dust Masses, PAH Abundances, and Starlight Intensities in the SINGS Galaxy Sample. ApJ663(2):866-894, DOI 10.1086/518306, astro-ph/0703213

Du X, Shapley AE, Reddy NA, Jones T, Stark DP, Steidel CC, Strom AL, Rudie GC, Erb DK, Ellis RS, Pettini M (2018) The Redshift Evolution of Rest-UV Spectroscopic Properties in Lyman-break Galaxies at $z \sim 2-4$. ApJ860(1):75, DOI 10.3847/1538-4357/aabfcf, 1803.05912

Dubois Y, Peirani S, Pichon C, Devriendt J, Gavazzi R, Welker C, Volonteri M (2016) The HORIZON-AGN simulation: morphological diversity of galaxies promoted by AGN feedback. MNRAS463(4):3948-3964, DOI 10.1093/mnras/stw2265, 1606.03086

Dugan Z, Gaibler V, Silk J (2017) Feedback by AGN Jets and Wide-angle Winds on a Galactic Scale. ApJ844(1):37, DOI 10.3847/1538-4357/aa7566, 1608.01370

Dumouchel F, Faure A, Lique F (2010) The rotational excitation of HCN and HNC by He: temperature dependence of the collisional rate coefficients. MNRAS406(4):2488-2492, DOI 10.1111/j.1365-2966. 2010.16826.x

Dunn JP, Bautista M, Arav N, Moe M, Korista K, Costantini E, Benn C, Ellison S, Edmonds D (2010) The Quasar Outflow Contribution to AGN Feedback: VLT Measurements of SDSS J0318-0600. ApJ709(2):611-631, DOI 10.1088/0004-637X/709/2/611, 0911. 3896

Dunn JP, Wasik B, Holtzclaw CL, Yenerall D, Bautista M, Arav N, Hayes D, Moe M, Ho LC, Harper Dutton S (2015) Determining the Locations of Dust Sources in FeLoBAL Quasars. ApJ808(1):94, DOI 10.1088/0004-637X/808/1/94, 1509.05060

Efstathiou G (2000) A model of supernova feedback in galaxy formation. MNRAS317(3):697-719, DOI 10.1046/j.1365-8711.2000.03665.x, astro-ph/0002245

El-Badry K, Wetzel A, Geha M, Hopkins PF, Kereš D, Chan TK, Faucher-Giguère CA (2016) Breathing FIRE: How Stellar Feedback Drives Radial Migration, Rapid Size Fluctuations, and Population Gradients in Low-mass Galaxies. ApJ820(2):131, DOI 10.3847/0004-637X/820/2/131, 1512.01235

El-Badry K, Ostriker EC, Kim CG, Quataert E, Weisz DR (2019) Evolution of supernovae-driven superbubbles with conduction and cooling. arXiv e-prints arXiv:1902.09547, 1902.09547

Elbaz D, Jahnke K, Pantin E, Le Borgne D, Letawe G (2009) Quasar induced galaxy formation: a new paradigm? A\&A507(3):1359-1374, DOI 10.1051/0004-6361/200912848, 0907.2923

Elitzur M, Shlosman I (2006) The AGN-obscuring Torus: The End of the "Doughnut" Paradigm? ApJLett648(2):L101-L104, DOI 10.1086/508158, astro-ph/0605686

Ellison SL, Thorp MD, Lin L, Pan HA, Bluck AFL, Scudder JM, Teimoorinia H, Sanchez SF, Sargent M (2019) The ALMaQUEST Survey: III. Scatter in the resolved star forming main sequence is primarily due to variations in star formation efficiency. arXiv e-prints arXiv:1911.11887, 1911.11887

Emerick A, Bryan GL, Mac Low MM, Côté B, Johnston KV, O’Shea BW (2018) Metal Mixing and Ejection in Dwarf Galaxies Are Dependent on Nucleosynthetic Source. ApJ869(2):94, DOI 10.3847/ 1538-4357/aaec7d, 1809.01167

Engelbracht CW, Kundurthy P, Gordon KD, Rieke GH, Kennicutt RC, Smith JDT, Regan MW, Makovoz D, Sosey M, Draine BT, Helou G, Armus L, Calzetti D, Meyer M, Bendo GJ, Walter F, Hollenbach D, Cannon JM, Murphy EJ, Dale DA, Buckalew BA, Sheth K (2006) Extended Mid-Infrared Aromatic Feature Emission in M82. ApJLett642(2):L127-L132, DOI 10.1086/504590, astro-ph/0603551

Erb DK (2015) Feedback in low-mass galaxies in the early Universe. Nature523(7559):169-176, DOI 10.1038/nature14454, 1507.02374

Erb DK, Quider AM, Henry AL, Martin CL (2012) Galactic Outflows in Absorption and Emission: Nearultraviolet Spectroscopy of Galaxies at 1 \&lt; z \&lt; 2. ApJ759(1):26, DOI 10.1088/0004-637X/759/ $1 / 26,1209.4903$

Erb DK, Steidel CC, Chen Y (2018) The Kinematics of Extended Ly $\alpha$ Emission in a Low-mass, Lowmetallicity Galaxy at $z=2.3$. ApJLett862(1):L10, DOI 10.3847/2041-8213/aacff6, 1807.00065

Espada D, Peck AB, Matsushita S, Sakamoto K, Henkel C, Iono D, Israel FP, Muller S, Petitpas G, Pihlström Y, Taylor GB, Trung DV (2010) Disentangling the Circumnuclear Environs of Centaurus A. II. On the Nature of the Broad Absorption Line. ApJ720(1):666-678, DOI 10.1088/0004-637X/ $720 / 1 / 666,1007.2061$

Everett JE, Zweibel EG, Benjamin RA, McCammon D, Rocks L, Gallagher I John S (2008) The Milky Way's Kiloparsec-Scale Wind: A Hybrid Cosmic-Ray and Thermally Driven Outflow. ApJ674(1):258-270, DOI 10.1086/524766, 0710.3712

Fabian AC (1999) The obscured growth of massive black holes. MNRAS308(4):L39-L43, DOI 10.1046/ 
j.1365-8711.1999.03017.x, astro-ph/9908064

Fabian AC (2012) Observational Evidence of Active Galactic Nuclei Feedback. ARA\&A50:455-489, DOI 10.1146/annurev-astro-081811-125521, 1204.4114

Faerman Y, Sternberg A, McKee CF (2017) Massive Warm/Hot Galaxy Coronae as Probed by UV/X-Ray Oxygen Absorption and Emission. I. Basic Model. ApJ835(1):52, DOI 10.3847/1538-4357/835/1/52, 1602.00689

Falgarone E, Puget JL (1995) The intermittency of turbulence in interstellar clouds: implications for the gas kinetic temperature and decoupling of heavy particles from the gas motions. A\&A293:840-852

Falgarone E, Zwaan MA, Godard B, Bergin E, Ivison RJ, Andreani PM, Bournaud F, Bussmann RS, Elbaz D, Omont A, Oteo I, Walter F (2017) Large turbulent reservoirs of cold molecular gas around high-redshift starburst galaxies. Nature548(7668):430-433, DOI 10.1038/nature23298, 1708.08851

Falstad N, González-Alfonso E, Aalto S, van der Werf PP, Fischer J, Veilleux S, Meléndez M, Farrah D, Smith HA (2015) Herschel spectroscopic observations of the compact obscured nucleus in $\mathrm{Zw}$ 049.057. A\&A580:A52, DOI 10.1051/0004-6361/201526114, 1505.06934

Falstad N, González-Alfonso E, Aalto S, Fischer J (2017) Inflowing gas onto a compact obscured nucleus in Arp 299A. Herschel spectroscopic studies of $\mathrm{H}_{2} \mathrm{O}$ and $\mathrm{OH}$. A\&A597:A105, DOI 10.1051/0004-6361/201629050, 1611.01071

Falstad N, Aalto S, Mangum JG, Costagliola F, Gallagher JS, González-Alfonso E, Sakamoto K, König S, Muller S, Evans AS, Privon GC (2018) Hidden molecular outflow in the LIRG Zw 049.057. A\&A609:A75, DOI 10.1051/0004-6361/201732088, 1711.05321

Falstad N, Hallqvist F, Aalto S, König S, Muller S, Aladro R, Combes F, Evans AS, Fuller GA, Gallagher JS, García-Burillo S, González-Alfonso E, Greve TR, Henkel C, Imanishi M, Izumi T, Mangum JG, Martín S, Privon GC, Sakamoto K, Veilleux S, van der Werf PP (2019) Hidden or missing outflows in highly obscured galaxy nuclei? A\&A623:A29, DOI 10.1051/0004-6361/201834586, 1901.06723

Fan L, Knudsen KK, Fogasy J, Drouart G (2018) ALMA Detections of CO Emission in the Most Luminous, Heavily Dust-obscured Quasars at z \&gt; 3. ApJLett856(1):L5, DOI 10.3847/2041-8213/ aab496, 1711. 10615

Fang T, Jiang X (2014) High Resolution X-Ray Spectroscopy of the Local Hot Gas along the 3C 273 Sightline. ApJLett785(2):L24, DOI 10.1088/2041-8205/785/2/L24, 1403. 2028

Farina EP, Falomo R, Scarpa R, Decarli R, Treves A, Kotilainen JK (2014) The extent of the Mg II absorbing circumgalactic medium of quasars. MNRAS441(1):886-899, DOI 10.1093/mnras/stu585, 1403.5559

Faucher-Giguère CA, Quataert E (2012) The physics of galactic winds driven by active galactic nuclei. MNRAS425(1):605-622, DOI 10.1111/j.1365-2966.2012.21512.x, 1204.2547

Feain IJ, Papadopoulos PP, Ekers RD, Middelberg E (2007) Dressing a Naked Quasar: Star Formation and Active Galactic Nucleus Feedback in HE 0450-2958. ApJ662(2):872-877, DOI 10.1086/518027, astro-ph/0703101

Fernández-Ontiveros JA, Dasyra KM, Hatziminaoglou E, Malkan MA, Pereira-Santaella M, Papachristou M, Spinoglio L, Combes F, Aalto S, Nagar N, Imanishi M, Andreani P, Ricci C, Slater R (2019) A $\mathrm{CO}$ molecular gas wind $340 \mathrm{pc}$ away from the Seyfert 2 nucleus in ESO 420-G13 probes an elusive radio jet. arXiv e-prints arXiv: $1911.00015,1911.00015$

Ferrara A, Viti S, Ceccarelli C (2016) The problematic growth of dust in high-redshift galaxies. MNRAS463(1):L112-L116, DOI 10.1093/mnrasl/slw165, 1606.07214

Ferrarese L, Merritt D (2000) A Fundamental Relation between Supermassive Black Holes and Their Host Galaxies. ApJLett539(1):L9-L12, DOI 10.1086/312838, astro-ph/0006053

Feruglio C, Maiolino R, Piconcelli E, Menci N, Aussel H, Lamastra A, Fiore F (2010) Quasar feedback revealed by giant molecular outflows. A\&A518:L155, DOI 10.1051/0004-6361/201015164, 1006. 1655

Feruglio C, Fiore F, Carniani S, Piconcelli E, Zappacosta L, Bongiorno A, Cicone C, Maiolino R, Marconi A, Menci N, Puccetti S, Veilleux S (2015) The multi-phase winds of Markarian 231: from the hot, nuclear, ultra-fast wind to the galaxy-scale, molecular outflow. A\&A583:A99, DOI 10.1051/0004-6361/201526020, 1503.01481

Feruglio C, Ferrara A, Bischetti M, Downes D, Neri R, Ceccarelli C, Cicone C, Fiore F, Gallerani S, Maiolino R, Menci N, Piconcelli E, Vietri G, Vignali C, Zappacosta L (2017) On the discovery of fast molecular gas in the UFO/BAL quasar APM $08279+5255$ at $z=3.912$. A\&A608:A30, DOI 10.1051/0004-6361/201731387, 1706.05527

Field GB, Steigman G (1971) Charge Transfer and Ionization Equilibrium in the Interstellar Medium. ApJ166:59, DOI 10.1086/150941 
Fierlinger KM, Burkert A, Ntormousi E, Fierlinger P, Schartmann M, Ballone A, Krause MGH, Diehl R (2016) Stellar feedback efficiencies: supernovae versus stellar winds. MNRAS456(1):710-730, DOI 10.1093/mnras/stv2699, 1511.05151

Filiz Ak N, Brandt WN, Hall PB, Schneider DP, Anderson SF, Gibson RR, Lundgren BF, Myers AD, Petitjean P, Ross NP, Shen Y, York DG, Bizyaev D, Brinkmann J, Malanushenko E, Oravetz DJ, Pan K, Simmons AE, Weaver BA (2012) Broad Absorption Line Disappearance on Multi-year Timescales in a Large Quasar Sample. ApJ757(2):114, DOI 10.1088/0004-637X/757/2/114, 1208.0836

Finkbeiner DP (2004) Microwave Interstellar Medium Emission Observed by the Wilkinson Microwave Anisotropy Probe. ApJ614(1):186-193, DOI 10.1086/423482, astro-ph/0311547

Finlator K, Davé R (2008) The origin of the galaxy mass-metallicity relation and implications for galactic outflows. MNRAS385(4):2181-2204, DOI 10.1111/j.1365-2966.2008.12991.x, 0704.3100

Finley H, Bouché N, Contini T, Epinat B, Bacon R, Brinchmann J, Cantalupo S, Erroz-Ferrer S, Marino RA, Maseda M, Richard J, Schroetter I, Verhamme A, Weilbacher PM, Wendt M, Wisotzki L (2017) Galactic winds with MUSE: A direct detection of Fe II* emission from a $z=1.29$ galaxy. A\&A605:A118, DOI 10.1051/0004-6361/201730428, 1701.07843

Fiore F, Feruglio C, Shankar F, Bischetti M, Bongiorno A, Brusa M, Carniani S, Cicone C, Duras F, Lamastra A, Mainieri V, Marconi A, Menci N, Maiolino R, Piconcelli E, Vietri G, Zappacosta L (2017) AGN wind scaling relations and the co-evolution of black holes and galaxies. A\&A601:A143, DOI 10.1051/0004-6361/201629478, 1702.04507

Fischer J, Sturm E, González-Alfonso E, Graciá-Carpio J, Hailey-Dunsheath S, Poglitsch A, Contursi A, Lutz D, Genzel R, Sternberg A, Verma A, Tacconi L (2010) Herschel-PACS spectroscopic diagnostics of local ULIRGs: Conditions and kinematics in Markarian 231. A\&A518:L41, DOI 10.1051/0004-6361/201014676, 1005.2213

Fischer J, Abel NP, González-Alfonso E, Dudley CC, Satyapal S, van Hoof PAM (2014) A Far-infrared Spectral Sequence of Galaxies: Trends and Models. ApJ795(2):117, DOI 10.1088/0004-637X/795/2/ $117,1409.2521$

Fluetsch A, Maiolino R, Carniani S, Marconi A, Cicone C, Bourne MA, Costa T, Fabian AC, Ishibashi W, Venturi G (2019) Cold molecular outflows in the local Universe and their feedback effect on galaxies. MNRAS483(4):4586-4614, DOI 10.1093/mnras/sty3449, 1805.05352

Forbes JC, Krumholz MR, Speagle JS (2019) Towards a radially resolved semi-analytic model for the evolution of disc galaxies tuned with machine learning. MNRAS487(3):3581-3606, DOI 10.1093/ mnras/stz1473, 1810.12919

Förster Schreiber NM, Genzel R, Lutz D, Sternberg A (2003) The Nature of Starburst Activity in M82. ApJ599(1):193-217, DOI 10.1086/379097, astro-ph/0309357

Förster Schreiber NM, Übler H, Davies RL, Genzel R, Wisnioski E, Belli S, Shimizu T, Lutz D, Fossati M, Herrera-Camus R, Mendel JT, Tacconi LJ, Wilman D, Beifiori A, Brammer GB, Burkert A, Carollo CM, Davies RI, Eisenhauer F, Fabricius M, Lilly SJ, Momcheva I, Naab T, Nelson EJ, Price SH, Renzini A, Saglia R, Sternberg A, van Dokkum P, Wuyts S (2019) The KMOS ${ }^{3 D}$ Survey: Demographics and Properties of Galactic Outflows at $z=0.6-2.7$. ApJ875(1):21, DOI 10.3847/1538-4357/ab0ca2, 1807.04738

Fotopoulou CM, Dasyra KM, Combes F, Salomé P, Papachristou M (2019) Complex molecular gas kinematics in the inner $5 \mathrm{kpc}$ of 4C12.50 as seen by ALMA. A\&A629:A30, DOI 10.1051/0004-6361/ 201834416, 1908.01011

Fox AJ, Ledoux C, Vreeswijk PM, Smette A, Jaunsen AO (2008) High-ion absorption in seven GRB host galaxies at $\mathrm{z}=2-4$. Evidence for both circumburst plasma and outflowing interstellar gas. A\&A491(1):189-207, DOI 10.1051/0004-6361:200810286, 0809. 3247

Fox AJ, Bordoloi R, Savage BD, Lockman FJ, Jenkins EB, Wakker BP, Bland-Hawthorn J, Hernandez S, Kim TS, Benjamin RA, Bowen DV, Tumlinson J (2015) Probing the Fermi Bubbles in U1traviolet Absorption: A Spectroscopic Signature of the Milky Way's Biconical Nuclear Outflow. ApJLett799(1):L7, DOI 10.1088/2041-8205/799/1/L7, 1412.1480

Fox AJ, Richter P, Ashley T, Heckman TM, Lehner N, Werk JK, Bordoloi R, Peeples MS (2019) The Mass Inflow and Outflow Rates of the Milky Way. arXiv e-prints arXiv:1909.05561, 1909.05561

Fujimoto S, Ouchi M, Ferrara A, Pallottini A, Ivison RJ, Behrens C, Gallerani S, Arata S, Yajima H, Nagamine K (2019) First Identification of 10-kpc Scale [CII] 158um Halos around Star-Forming Galaxies at $\mathrm{Z}=5-7$. arXiv e-prints arXiv:1902.06760, 1902.06760

Fukugita M (2011) Global amount of dust in the universe. arXiv e-prints arXiv:1103.4191, 1103.4191

Fukugita M, Ménard B (2015) The Nature of Damped Lyman- $\alpha$ and Mg II Absorbers Explored through Their Dust Contents. ApJ799(2):195, DOI 10.1088/0004-637X/799/2/195, 1403.4339 
Fukui Y, Torii K, Onishi T, Yamamoto H, Okamoto R, Hayakawa T, Tachihara K, Sano H (2015) Optically Thick H I Dominant in the Local Interstellar Medium: An Alternative Interpretation to "Dark Gas". ApJ798(1):6, DOI 10.1088/0004-637X/798/1/6, 1403.0999

Gabor JM, Bournaud F (2014) Active galactic nuclei-driven outflows without immediate quenching in simulations of high-redshift disc galaxies. MNRAS441(2):1615-1627, DOI 10.1093/mnras/stu677, 1402.4482

Gaibler V, Khochfar S, Krause M, Silk J (2012) Jet-induced star formation in gas-rich galaxies. MNRAS425(1):438-449, DOI 10.1111/j.1365-2966.2012.21479.x, 1111.4478

Gall C, Hjorth J (2018) Maximally Dusty Star-forming Galaxies: Supernova Dust Production and Recycling in Local Group and High-redshift Galaxies. ApJ868(1):62, DOI 10.3847/1538-4357/aae520, 1809.11032

Gall C, Hjorth J, Watson D, Dwek E, Maund JR, Fox O, Leloudas G, Malesani D, Day-Jones AC (2014) Rapid formation of large dust grains in the luminous supernova 2010j1. Nature511(7509):326-329, DOI 10.1038 /nature $13558,1407.4447$

Gallagher R, Maiolino R, Belfiore F, Drory N, Riffel R, Riffel RA (2019) Widespread star formation inside galactic outflows. MNRAS485(3):3409-3429, DOI 10.1093/mnras/stz564, 1806.03311

Gallagher SC, Brandt WN, Chartas G, Garmire GP, Sambruna RM (2002) X-Raying the Ultraluminous Infrared Starburst Galaxy and Broad Absorption Line QSO Markarian 231 with Chandra. ApJ569(2):655-670, DOI 10.1086/339171, astro-ph/0112257

Gallagher SC, Schmidt GD, Smith PS, Brand t WN, Chartas G, Hylton S, Hines DC, Brotherton MS (2005) Variation in the Scattering Shroud Surrounding Markarian 231. ApJ633(1):71-85, DOI 10 1086/449305, astro-ph/0506616

Gallerani S, Pallottini A, Feruglio C, Ferrara A, Maiolino R, Vallini L, Riechers DA, Pavesi R (2018) ALMA suggests outflows in $z \sim 5.5$ galaxies. MNRAS473(2):1909-1917, DOI 10.1093/mnras/ stx2458, 1604.05714

Galliano E, Alloin D (2002) Near-IR 2D-spectroscopy of the 4"x 4" region around the Active Galactic Nucleus of NGC 1068 with ISAAC/VLT. A\&A393:43-56, DOI 10.1051/0004-6361:20020906, astro-ph/0207010

Galliano F, Galametz M, Jones AP (2018) The Interstellar Dust Properties of Nearby Galaxies. ARA\&A56:673-713, DOI 10.1146/annurev-astro-081817-051900, 1711.07434

Gallimore JF, Baum SA, O'Dea CP (1997) A direct image of the obscuring disk surrounding an active galactic nucleus. Nature388(6645):852-854, DOI 10.1038/42201, astro-ph/9708062

Gallimore JF, Elitzur M, Maiolino R, Marconi A, O'Dea CP, Lutz D, Baum SA, Nikutta R, Impellizzeri CMV, Davies R, Kimball AE, Sani E (2016) High-velocity Bipolar Molecular Emission from an AGN Torus. ApJLett829(1):L7, DOI 10.3847/2041-8205/829/1/L7, 1608.02210

García-Burillo S, Martín-Pintado J, Fuente A, Neri R (2001) SiO Chimneys and Supershells in M82. ApJLett563(1):L27-L30, DOI 10.1086/338391, astro-ph/0201009

García-Burillo S, Usero A, Fuente A, Martín-Pintado J, Boone F, Aalto S, Krips M, Neri R, Schinnerer E, Tacconi LJ (2010) Molecular gas chemistry in AGN. II. High-resolution imaging of SiO emission in NGC 1068: shocks or XDR? A\&A519:A2, DOI 10.1051/0004-6361/201014539, 1005. 1263

García-Burillo S, Combes F, Usero A, Aalto S, Krips M, Viti S, Alonso-Herrero A, Hunt LK, Schinnerer E, Baker AJ, Boone F, Casasola V, Colina L, Costagliola F, Eckart A, Fuente A, Henkel C, Labiano A, Martín S, Márquez I, Muller S, Planesas P, Ramos Almeida C, Spaans M, Tacconi LJ, van der Werf PP (2014) Molecular line emission in NGC 1068 imaged with ALMA. I. An AGN-driven outflow in the dense molecular gas. A\&A567:A125, DOI 10.1051/0004-6361/201423843, 1405.7706

García-Burillo S, Combes F, Usero A, Aalto S, Colina L, Alonso-Herrero A, Hunt LK, Arribas S, Costagliola F, Labiano A, Neri R, Pereira-Santaella M, Tacconi LJ, van der Werf PP (2015) High-resolution imaging of the molecular outflows in two mergers: ASTROBJ $_{i}$ IRAS $17208-0014_{i} /$ ASTROBJ $_{i}$ and ¡ASTROBJ ${ }_{i}$ NGC 1614 $_{i} /$ ASTROBJ $_{i}$. A\&A580:A35, DOI 10.1051/0004-6361/201526133, 1505. 04705

García-Burillo S, Combes F, Ramos Almeida C, Usero A, Krips M, Alonso-Herrero A, Aalto S, Casasola V, Hunt LK, Martín S, Viti S, Colina L, Costagliola F, Eckart A, Fuente A, Henkel C, Márquez I, Neri R, Schinnerer E, Tacconi LJ, van der Werf PP (2016) ALMA Resolves the Torus of NGC 1068: Continuum and Molecular Line Emission. ApJLett823(1):L12, DOI 10.3847/2041-8205/823/1/L12, 1604.00205

García-Burillo S, Viti S, Combes F, Fuente A, Usero A, Hunt LK, Martín S, Krips M, Aalto S, Aladro R, Ramos Almeida C, Alonso-Herrero A, Casasola V, Henkel C, Querejeta M, Neri R, Costagliola F, Tacconi LJ, van der Werf PP (2017) ALMA imaging of $\mathrm{C}_{2} \mathrm{H}$ emission in the disk of ${ }_{i} \mathrm{ASTROBJ}_{i} \mathrm{NGC}$ 
1068;/ASTROBJ ${ }_{i}$. A\&A608:A56, DOI 10.1051/0004-6361/201731862, 1709.05895

Garcia-Burillo S, Combes F, Ramos Almeida C, Usero A, Alonso-Herrero A, Hunt LK, Rouan D, Aalto S, Querejeta M, Viti S, van der Werf PP, Fuente A, Colina L, Martin-Pintado J, Henkel C, Martin S, Gratadour D, Neri R, Tacconi LJ (2019) ALMA images the many faces of the NGC1068 torus and its surroundings. arXiv e-prints arXiv:1909.00675, 1909.00675

Gaspari M, Temi P, Brighenti F (2017) Raining on black holes and massive galaxies: the top-down multiphase condensation model. MNRAS466(1):677-704, DOI 10.1093/mnras/stw3108, 1608.08216

Gatkine P, Veilleux S, Cucchiara A (2019) The CGM-GRB Study. I. Uncovering The CircumGalactic Medium around GRB hosts at redshifts 2-6. arXiv e-prints arXiv:1907.05903, 1907.05903

Gatuzz E, García J, Kallman TR, Mendoza C, Gorczyca TW (2015) ISMabs: A Comprehensive X-Ray Absorption Model for the Interstellar Medium. ApJ800(1):29, DOI 10.1088/0004-637X/800/1/29, 1412.3813

Gatuzz E, García JA, Kallman TR, Mendoza C (2016) Oxygen, neon, and iron X-ray absorption in the local interstellar medium. A\&A588:A111, DOI 10.1051/0004-6361/201527752, 1602.06955

Geach JE, Hickox RC, Diamond-Stanic AM, Krips M, Rudnick GH, Tremonti CA, Sell PH, Coil AL, Moustakas J (2014) Stellar feedback as the origin of an extended molecular outflow in a starburst galaxy. Nature516(7529):68-70, DOI 10.1038/nature14012, 1412. 1091

Gebhardt K, Bender R, Bower G, Dressler A, Faber SM, Filippenko AV, Green R, Grillmair C, Ho LC, Kormendy J, Lauer TR, Magorrian J, Pinkney J, Richstone D, Tremaine S (2000) A Relationship between Nuclear Black Hole Mass and Galaxy Velocity Dispersion. ApJLett539(1):L13-L16, DOI 10.1086/312840, astro-ph/0006289

Gentry ES, Krumholz MR, Dekel A, Madau P (2017) Enhanced momentum feedback from clustered supernovae. MNRAS465(2):2471-2488, DOI 10.1093/mnras/stw2746, 1606.01242

Gentry ES, Krumholz MR, Madau P, Lupi A (2019) The momentum budget of clustered supernova feedback in a 3D, magnetized medium. MNRAS483(3):3647-3658, DOI 10.1093/mnras/sty3319, 1802.06860

Genzel R, Förster Schreiber NM, Rosario D, Lang P, Lutz D, Wisnioski E, Wuyts E, Wuyts S, Bandara K, Bender R, Berta S, Kurk J, Mendel JT, Tacconi LJ, Wilman D, Beifiori A, Brammer G, Burkert A, Buschkamp P, Chan J, Carollo CM, Davies R, Eisenhauer F, Fabricius M, Fossati M, Kriek M, Kulkarni S, Lilly SJ, Mancini C, Momcheva I, Naab T, Nelson EJ, Renzini A, Saglia R, Sharples RM, Sternberg A, Tacchella S, van Dokkum P (2014) Evidence for Wide-spread Active Galactic Nucleus-driven Outflows in the Most Massive $z \sim 1-2$ Star-forming Galaxies. ApJ796(1):7, DOI 10.1088/0004-637X/796/1/7, 1406.0183

George RD, Ivison RJ, Smail I, Swinbank AM, Hopwood R, Stanley F, Swinyard BM, Valtchanov I, Werf PPvd (2014) Herschel reveals a molecular outflow in a $\mathrm{z}=2.3$ ULIRG. MNRAS442(2):1877-1883, DOI 10.1093/mnras/stu967, 1402.6320

Georgy C, Walder R, Folini D, Bykov A, Marcowith A, Favre JM (2013) Circumstellar medium around rotating massive stars at solar metallicity. A\&A559:A69, DOI 10.1051/0004-6361/201321226, 1309. 1360

Gilli R, Maiolino R, Marconi A, Risaliti G, Dadina M, Weaver KA, Colbert EJM (2000) The variability of the Seyfert galaxy NGC 2992: the case for a revived AGN. A\&A355:485-498, astro-ph/0001107

Ginolfi M, Jones GC, Bethermin M, Fudamoto Y, Loiacono F, Fujimoto S, Le Fevre O, Faisst A, Schaerer D, Cassata P, Silverman JD, Yan L, Capak P, Bardelli S, Boquien M, Carraro R, Dessauges-Zavadsky M, Giavalisco M, Gruppioni C, Ibar E, Khusanova Y, Lemaux BC, Maiolino R, Narayanan D, Oesch P, Pozzi F, Rodighiero G, Talia M, Toft S, Vallini L, Vergani D, Zamorani G (2019) The ALPINE-ALMA [CII] survey: Star-formation-driven outflows and circumgalactic enrichment in the early Universe. arXiv e-prints arXiv:1910.04770, 1910.04770

Giovanardi C, Natta A, Palla F (1987) Numerical FITS to the electron impact transition rate coefficients for atomic hydrogen as a function of electron temperature. A\&AS70(2):269-280

Girichidis P, Naab T, Walch S, Hanasz M, Mac Low MM, Ostriker JP, Gatto A, Peters T, Wünsch R, Glover SCO, Klessen RS, Clark PC, Baczynski C (2016) Launching Cosmic-Ray-driven Outflows from the Magnetized Interstellar Medium. ApJLett816(2):L19, DOI 10.3847/2041-8205/816/2/L19, 1509.07247

Girichidis P, Naab T, Hanasz M, Walch S (2018) Cooler and smoother - the impact of cosmic rays on the phase structure of galactic outflows. MNRAS479(3):3042-3067, DOI 10.1093/mnras/sty1653, 1805.09333

Giustini M, Proga D (2019) A global view of the inner accretion/ejection flow around super massive black holes: radiation driven accretion disk winds in a physical context. arXiv e-prints arXiv:1904.07341, 
1904.07341

Glenn J, Rangwala N, Maloney PR, Kamenetzky JR (2015) NGC 1266: Characterization of the Nuclear Molecular Gas in an Unusual SB0 Galaxy. ApJ800(2):105, DOI 10.1088/0004-637X/800/2/105, 1502.02041

Glover SC, Savin DW, Jappsen AK (2006) Cosmological Implications of the Uncertainty in $\mathrm{H}^{-}$Destruction Rate Coefficients. ApJ640(2):553-568, DOI 10.1086/500166, astro-ph/0506221

Glover SCO, Clark PC, Micic M, Molina F (2015) Modelling [C I] emission from turbulent molecular clouds. MNRAS448(2):1607-1627, DOI 10.1093/mnras/stu2699, 1403. 3530

Goicoechea JR, Cernicharo J (2002) Far-Infrared OH Fluorescent Emission in Sagittarius B2. ApJLett576(1):L77-L81, DOI 10.1086/343062, astro-ph/0207498

Goicoechea JR, Cernicharo J, Lerate MR, Daniel F, Barlow MJ, Swinyard BM, Lim TL, Viti S, Yates J (2006) Far-Infrared Excited Hydroxyl Lines from Orion KL Outflows. ApJLett641(1):L49-L52, DOI 10.1086/503799, astro-ph/0603077

Goicoechea JR, Pety J, Gerin M, Hily-Blant P, Le Bourlot J (2009) The ionization fraction gradient across the Horsehead edge: an archetype for molecular clouds. A\&A498(3):771-783, DOI 10.1051/0004-6361/200811496, 0902.2748

Goicoechea JR, Chavarría L, Cernicharo J, Neufeld DA, Vavrek R, Bergin EA, Cuadrado S, Encrenaz P, Etxaluze M, Melnick GJ, Polehampton E (2015) Herschel Far-infrared Spectral-mapping of Orion BN/KL Outflows: Spatial Distribution of Excited CO, $\mathrm{H}_{2} \mathrm{O}, \mathrm{OH}, \mathrm{O}$, and $\mathrm{C}^{+}$in Shocked Gas. ApJ799(1):102, DOI 10.1088/0004-637X/799/1/102, 1411.2930

Goicoechea JR, Santa-Maria MG, Teyssier D, Cernicharo J, Gerin M, Pety J (2018) High-velocity hot CO emission close to Sgr A*. Herschel/HIFI submillimeter spectral survey toward Sgr A*. A\&A616:L1, DOI 10.1051/0004-6361/201833684, 1807.07459

Goldsmith PF, Kauffmann J (2017) Electron Excitation of High Dipole Moment Molecules Re-examined. ApJ841(1):25, DOI 10.3847/1538-4357/aa6f12, 1708.07553

Goldsmith PF, Langer WD, Pineda JL, Velusamy T (2012) Collisional Excitation of the [C II] Fine Structure Transition in Interstellar Clouds. ApJSupp.203(1):13, DOI 10.1088/0067-0049/203/1/13, 1209.4536

González-Alfonso E, Sakamoto K (2019) The Greenhouse Effect in Buried Galactic Nuclei and the Resonant HCN Vibrational Emission. ApJ882(2):153, DOI 10.3847/1538-4357/ab3a32, 1908.04058

González-Alfonso E, Fischer J, Graciá-Carpio J, Sturm E, Hailey-Dunsheath S, Lutz D, Poglitsch A, Contursi A, Feuchtgruber H, Veilleux S, Spoon HWW, Verma A, Christopher N, Davies R, Sternberg A, Genzel R, Tacconi L (2012) Herschel/PACS spectroscopy of NGC 4418 and Arp 220: $\mathrm{H}_{2} \mathrm{O}, \mathrm{H}_{2}{ }^{18} \mathrm{O}$, $\mathrm{OH},{ }^{18} \mathrm{OH}, \mathrm{O} \mathrm{I}, \mathrm{HCN}$, and $\mathrm{NH}_{3}$. A\&A541:A4, DOI 10.1051/0004-6361/201118029, 1109.1118

González-Alfonso E, Fischer J, Bruderer S, Müller HSP, Graciá-Carpio J, Sturm E, Lutz D, Poglitsch A, Feuchtgruber H, Veilleux S, Contursi A, Sternberg A, Hailey-Dunsheath S, Verma A, Christopher N, Davies R, Genzel R, Tacconi L (2013) Excited $\mathrm{OH}^{+}, \mathrm{H}_{2} \mathrm{O}^{+}$, and $\mathrm{H}_{3} \mathrm{O}^{+}$in NGC 4418 and Arp 220. A\&A550:A25, DOI 10.1051/0004-6361/201220466, 1211.5064

González-Alfonso E, Fischer J, Graciá-Carpio J, Falstad N, Sturm E, Meléndez M, Spoon HWW, Verma A, Davies RI, Lutz D, Aalto S, Polisensky E, Poglitsch A, Veilleux S, Contursi A (2014) The Mrk 231 molecular outflow as seen in OH. A\&A561:A27, DOI 10.1051/0004-6361/201321709, 1310. 3074

González-Alfonso E, Armus L, Carrera FJ, Charmandaris V, Efstathiou A, Egami E, Fernández-Ontiveros JA, Fischer J, Granato GL, Gruppioni C, Hatziminaoglou E, Imanishi M, Isobe N, Kaneda H, KozielWierzbowska D, Malkan MA, Martín-Pintado J, Mateos S, Matsuhara H, Miniutti G, Nakagawa T, Pozzi F, Rico-Villas F, Rodighiero G, Roelfsema P, Spinoglio L, Spoon HWW, Sturm E, van der Tak F, Vignali C, Wang L (2017a) Feedback and Feeding in the Context of Galaxy Evolution with SPICA: Direct Characterisation of Molecular Outflows and Inflows. PASA34:e054, DOI 10.1017/pasa.2017. 46, 1710.02356

González-Alfonso E, Fischer J, Spoon HWW, Stewart KP, Ashby MLN, Veilleux S, Smith HA, Sturm E, Farrah D, Falstad N, Meléndez M, Graciá-Carpio J, Janssen AW, Lebouteiller V (2017b) Molecular Outflows in Local ULIRGs: Energetics from Multitransition OH Analysis. ApJ836(1):11, DOI 10. 3847/1538-4357/836/1/11, 1612.08181

González-Alfonso E, Fischer J, Bruderer S, Ashby MLN, Smith HA, Veilleux S, Müller HSP, Stewart KP, Sturm E (2018) Outflowing $\mathrm{OH}^{+}$in Markarian 231: The Ionization Rate of the Molecular Gas. ApJ857(1):66, DOI 10.3847/1538-4357/aab6b8, 1803.04690

Gould RJ, Salpeter EE (1963) The Interstellar Abundance of the Hydrogen Molecule. I. Basic Processes. ApJ138:393, DOI 10.1086/147654

Gowardhan A, Spoon H, Riechers DA, González-Alfonso E, Farrah D, Fischer J, Darling J, Fergulio C, 
Afonso J, Bizzocchi L (2018) The Dual Role of Starbursts and Active Galactic Nuclei in Driving Extreme Molecular Outflows. ApJ859(1):35, DOI 10.3847/1538-4357/aabccc, 1804.03147

Gravity Collaboration, Abuter R, Amorim A, Bauböck M, Berger JP, Bonnet H, Brand ner W, Clénet Y, Coudé Du Foresto V, de Zeeuw PT, Dexter J, Duvert G, Eckart A, Eisenhauer F, Förster Schreiber NM, Garcia P, Gao F, Gendron E, Genzel R, Gerhard O, Gillessen S, Habibi M, Haubois X, Henning T, Hippler S, Horrobin M, Jiménez-Rosales A, Jocou L, Kervella P, Lacour S, Lapeyrère V, Le Bouquin JB, Léna P, Ott T, Paumard T, Perraut K, Perrin G, Pfuhl O, Rabien S, Rodriguez Coira G, Rousset G, Scheithauer S, Sternberg A, Straub O, Straubmeier C, Sturm E, Tacconi LJ, Vincent F, von Fellenberg S, Waisberg I, Widmann F, Wieprecht E, Wiezorrek E, Woillez J, Yazici S (2019) A geometric distance measurement to the Galactic center black hole with $0.3 \%$ uncertainty. A\&A625:L10, DOI 10.1051/ 0004-6361/201935656

Grenier IA, Black JH, Strong AW (2015) The Nine Lives of Cosmic Rays in Galaxies. ARA\&A53:199_ 246, DOI 10.1146/annurev-astro-082214-122457

Gronke M (2017) Modeling 237 Lyman- $\alpha$ spectra of the MUSE-Wide survey. A\&A608:A139, DOI 10 1051/0004-6361/201731791, 1709.07008

Gronke M, Oh SP (2018) The growth and entrainment of cold gas in a hot wind. MNRAS480(1):L111L115, DOI 10.1093/mnrasl/sly131, 1806.02728

Gronke M, Oh SP (2019) How cold gas continuously entrains mass and momentum from a hot wind. arXiv e-prints arXiv:1907.04771, 1907.04771

Gronke M, Girichidis P, Naab T, Walch S (2018) The Imprint of Cosmic Ray Driven Outflows on Lyman- $\alpha$ Spectra. ApJLett862(1):L7, DOI 10.3847/2041-8213/aad286, 1805. 12251

Grønnow A, Tepper-García T, Bland -Hawthorn J (2018) Magnetic Fields in the Galactic Halo Restrict Fountain-driven Recycling and Accretion. ApJ865(1):64, DOI 10.3847/1538-4357/aada0e, 1805.03903

Guillard P, Ogle PM, Emonts BHC, Appleton PN, Morganti R, Tadhunter C, Oosterloo T, Evans DA, Evans AS (2012) Strong Molecular Hydrogen Emission and Kinematics of the Multiphase Gas in Radio Galaxies with Fast Jet-driven Outflows. ApJ747(2):95, DOI 10.1088/0004-637X/747/2/95, 1201. 1503

Gültekin K, Richstone DO, Gebhardt K, Lauer TR, Tremaine S, Aller MC, Bender R, Dressler A, Faber SM, Filippenko AV, Green R, Ho LC, Kormendy J, Magorrian J, Pinkney J, Siopis C (2009) The M- $\sigma$ and M-L Relations in Galactic Bulges, and Determinations of Their Intrinsic Scatter. ApJ698(1):198221, DOI 10.1088/0004-637X/698/1/198, 0903. 4897

Guo F, Mathews WG (2012) The Fermi Bubbles. I. Possible Evidence for Recent AGN Jet Activity in the Galaxy. ApJ756(2):181, DOI 10.1088/0004-637X/756/2/181, 1103.0055

Hafen Z, Faucher-Giguère CA, Anglés-Alcázar D, Stern J, Kereš D, Hummels C, Esmerian C, GarrisonKimmel S, El-Badry K, Wetzel A, Chan TK, Hopkins PF, Murray N (2019) The origins of the circumgalactic medium in the FIRE simulations. MNRAS488(1):1248-1272, DOI 10.1093/mnras/stz1773, 1811.11753

Hailey-Dunsheath S, Nikola T, Stacey GJ, Oberst TE, Parshley SC, Benford DJ, Staguhn JG, Tucker CE (2010) Detection of the $158 \mu \mathrm{m}$ [C II] Transition at $\mathrm{z}=1.3$ : Evidence for a Galaxy-wide Starburst. ApJLett714(1):L162-L166, DOI 10.1088/2041-8205/714/1/L162, 1003. 2174

Hamann F, Zakamska NL, Ross N, Paris I, Alexandroff RM, Villforth C, Richards GT, Herbst H, Brandt WN, Cook B, Denney KD, Greene JE, Schneider DP, Strauss MA (2017) Extremely red quasars in BOSS. MNRAS464(3):3431-3463, DOI 10.1093/mnras/stw2387, 1609.07241

Hamann F, Herbst H, Paris I, Capellupo D (2019) On the structure and energetics of quasar broad absorption-line outflows. MNRAS483(2):1808-1828, DOI 10.1093/mnras/sty2900, 1810.03686

Hamer S, Salomé P, Combes F, Salomé Q (2015) MUSE discovers perpendicular arcs in the inner filament of Centaurus A. A\&A575:L3, DOI 10.1051/0004-6361/201424808, 1409.7700

Hao L, Spoon HWW, Sloan GC, Marshall JA, Armus L, Tielens AGGM, Sargent B, van Bemmel IM, Charmandaris V, Weedman DW, Houck JR (2005) The Detection of Silicate Emission from Quasars at 10 and 18 Microns. ApJLett625(2):L75-L78, DOI 10.1086/431227, astro-ph/0504423

Harada N (2018) High-Temperature Chemistry in External Galaxies. In: Cunningham M, Millar T, Aikawa Y (eds) IAU Symposium, IAU Symposium, vol 332, pp 25-36, DOI 10.1017/S1743921317006755, 1708.07269

Harada N, Sakamoto K, Martín S, Aalto S, Aladro R, Sliwa K (2018) ALMA Astrochemical Observations of the Infrared-luminous Merger NGC 3256. ApJ855(1):49, DOI 10.3847/1538-4357/aaaa70, 1801. Q5941

Harris GLH, Rejkuba M, Harris WE (2010) The Distance to NGC 5128 (Centaurus A). PASA27(4):457- 
462, DOI 10.1071/AS09061, 0911.3180

Harris J, Zaritsky D (2009) The Star Formation History of the Large Magellanic Cloud. AJ138(5):12431260, DOI 10.1088/0004-6256/138/5/1243, 0908. 1422

Harrison CM (2017) Impact of supermassive black hole growth on star formation. Nature Astronomy 1:0165, DOI 10.1038/s41550-017-0165, 1703.06889

Harrison CM, Costa T, Tadhunter CN, Flütsch A, Kakkad D, Perna M, Vietri G (2018) AGN outflows and feedback twenty years on. Nature Astronomy 2:198-205, DOI 10.1038/s41550-018-0403-6, 1802. 10306

Hartwig T, Volonteri M, Dashyan G (2018) Active galactic nucleus outflows in galaxy discs. MNRAS476(2):2288-2307, DOI 10.1093/mnras/sty229, 1707.03826

Hawley JF, Krolik JH (2006) Magnetically Driven Jets in the Kerr Metric. ApJ641(1):103-116, DOI 10.1086/500385, astro-ph/0512227

He Z, Wang T, Liu G, Wang H, Bian W, Tchernyshyov K, Mou G, Xu Y, Zhou H, Green R, Xu J (2019) The properties of broad absorption line outflows based on a large sample of quasars. Nature Astronomy $\mathrm{p}$ 197, DOI 10.1038/s41550-018-0669-8, 1812 .08982

Heckman TM, Borthakur S (2016) The Implications of Extreme Outflows from Extreme Starbursts. ApJ822(1):9, DOI 10.3847/0004-637X/822/1/9, 1603.03036

Heckman TM, Thompson TA (2017) A Brief Review of Galactic Winds. arXiv e-prints arXiv:1701.09062, 1701.09062

Heckman TM, Lehnert MD, Strickland DK, Armus L (2000) Absorption-Line Probes of Gas and Dust in Galactic Superwinds. ApJSupp.129(2):493-516, DOI 10.1086/313421, astro-ph/0002526

Heckman TM, Borthakur S, Overzier R, Kauffmann G, Basu-Zych A, Leitherer C, Sembach K, Martin DC, Rich RM, Schiminovich D, Seibert M (2011) Extreme Feedback and the Epoch of Reionization: Clues in the Local Universe. ApJ730(1):5, DOI 10.1088/0004-637X/730/1/5, 1101.4219

Heckman TM, Alexandroff RM, Borthakur S, Overzier R, Leitherer C (2015) The Systematic Properties of the Warm Phase of Starburst-Driven Galactic Winds. ApJ809(2):147, DOI 10.1088/0004-637X/ 809/2/147, 1507.05622

Heesen V, Rafferty DA, Horneffer A, Beck R, Basu A, Westcott J, Hindson L, Brinks E, Chyzy KT, Scaife AMM, Brüggen M, Heald G, Fletcher A, Horellou C, Tabatabaei FS, Paladino R, Nikiel-Wroczyński B, Hoeft M, Dettmar RJ (2018) Exploring the making of a galactic wind in the starbursting dwarf irregular galaxy IC 10 with LOFAR. MNRAS476(2):1756-1764, DOI 10.1093/mnras/sty325, 1802 . 01463

Heesen V, Whitler L, Schmidt P, Miskolczi A, Sridhar SS, Horellou C, Beck R, Gürkan G, Scannapieco E, Brüggen M, Heald GH, Krause M, Paladino R, Nikiel-Wroczyński B, Wilber A, Dettmar RJ (2019) Warped diffusive radio halo around the quiescent spiral edge-on galaxy NGC 4565. A\&A628:L3, DOI 10.1051/0004-6361/201936046, 1907.07076

Heintz KE, Watson D, Jakobsson P, Fynbo JPU, Bolmer J, Arabsalmani M, Cano Z, Covino S, D’Elia V, Gomboc A, Japelj J, Kaper L, Krogager JK, Pugliese G, Sánchez-Ramírez R, Selsing J, Sparre M, Tanvir NR, Thöne CC, de Ugarte Postigo A, Vergani SD (2018) Highly ionized metals as probes of the circumburst gas in the natal regions of gamma-ray bursts. MNRAS479(3):3456-3476, DOI $10.1093 / \mathrm{mnras} / \mathrm{sty} 1447,1806.01296$

Henden NA, Puchwein E, Shen S, Sijacki D (2018) The FABLE simulations: a feedback model for galaxies, groups, and clusters. MNRAS479(4):5385-5412, DOI 10.1093/mnras/sty1780, 1804.05064

Henshaw JD, Longmore SN, Kruijssen JMD, Davies B, Bally J, Barnes A, Battersby C, Burton M, Cunningham MR, Dale JE, Ginsburg A, Immer K, Jones PA, Kendrew S, Mills EAC, Molinari S, Moore TJT, Ott J, Pillai T, Rathborne J, Schilke P, Schmiedeke A, Testi L, Walker D, Walsh A, Zhang Q (2016) Molecular gas kinematics within the central $250 \mathrm{pc}$ of the Milky Way. MNRAS457(3):26752702, DOI 10.1093/mnras/stw121, 1601.03732

Herrera-Camus R, Janssen A, Sturm E, Lutz D, Veilleux S, Davies R, Shimizu T, González-Alfonso E, Rupke DSN, Tacconi L, Genzel R, Cicone C, Maiolino R, Contursi A, Graciá-Carpio J (2019a) AGN feedback in a galaxy merger: Multi-phase, galaxy-scale outflows including a fast molecular gas blob $\sim 6 \mathrm{kpc}$ away from IRAS F08572+3915. arXiv e-prints arXiv:1911.06326, 1911.06326

Herrera-Camus R, Sturm E, Graciá-Carpio J, Veilleux S, Shimizu T, Lutz D, Stone M, González-Alfonso E, Davies R, Fischer J, Genzel R, Maiolino R, Sternberg A, Tacconi L, Verma A (2019b) Molecular Gas Inflows and Outflows in Ultraluminous Infrared Galaxies at $z \sim 0.2$ and one QSO at $z=6.1$. arXiv e-prints arXiv:1912.05548, 1912.05548

Herrera-Camus R, Tacconi L, Genzel R, Förster Schreiber N, Lutz D, Bolatto A, Wuyts S, Renzini A, Lilly S, Belli S, Übler H, Shimizu T, Davies R, Sturm E, Combes F, Freundlich J, García-Burillo S, Cox P, 
Burkert A, Naab T, Colina L, Saintonge A, Cooper M, Feruglio C, Weiss A (2019c) Molecular and Ionized Gas Phases of an AGN-driven Outflow in a Typical Massive Galaxy at $z \approx 2$. ApJ871(1):37, DOI 10.3847/1538-4357/aaf6a7, 1807.07074

Heywood I, Camilo F, Cotton WD, Yusef-Zadeh F, Abbott TD, Adam RM, Aldera MA, Bauermeister EF, Booth RS, Botha AG, Botha DH, Brederode LRS, Brits ZB, Buchner SJ, Burger JP, Chalmers JM, Cheetham T, de Villiers D, Dikgale-Mahlakoana MA, du Toit LJ, Esterhuyse SWP, Fanaroff BL, Foley AR, Fourie DJ, Gamatham RRG, Goedhart S, Gounden S, Hlakola MJ, Hoek CJ, Hokwana A, Horn DM, Horrell JMG, Hugo B, Isaacson AR, Jonas JL, Jordaan JDBL, Joubert AF, Józsa GIG, Julie RPM, Kapp FB, Kenyon JS, Kotzé PPA, Kriel H, Kusel TW, Lehmensiek R, Liebenberg D, Loots A, Lord RT, Lunsky BM, Macfarlane PS, Magnus LG, Magozore CM, Mahgoub O, Main JPL, Malan JA, Malgas RD, Manley JR, Maree MDJ, Merry B, Millenaar R, Mnyandu N, Moeng IPT, Monama TE, Mphego MC, New WS, Ngcebetsha B, Oozeer N, Otto AJ, Passmoor SS, Pate AA, Peens-Hough A, Perkins SJ, Ratcliffe SM, Renil R, Rust A, Salie S, Schwardt LC, Serylak M, Siebrits R, Sirothia SK, Smirnov OM, Sofeya L, Swart PS, Tasse C, Taylor DT, Theron IP, Thorat K, Tiplad AJ, Tshongweni S, van Balla TJ, van der Byl A, van der Merwe C, van Dyk CL, Van Rooyen R, Van Tonder V, Van Wyk R, Wallace BH, Welz MG, Williams LP (2019) Inflation of 430-parsec bipolar radio bubbles in the Galactic Centre by an energetic event. arXiv e-prints arXiv:1909.05534, 1909.05534

Hinkle JT, Veilleux S, Rupke DSN (2019) Ionization Mechanisms in Quasar Outflows. ApJ881(1):31, DOI 10.3847/1538-4357/ab2bfa, 1906. 10249

Hirashita H, Lin CY (2018) Dust abundance and grain size in galaxy halos. arXiv e-prints arXiv: $1804.00848,1804.00848$

Hodges-Kluck E, Bregman JN (2014) Detection of Ultraviolet Halos around Highly Inclined Galaxies. ApJ789(2):131, DOI 10.1088/0004-637X/789/2/131, 1401.4170

Hodges-Kluck E, Cafmeyer J, Bregman JN (2016a) Ultraviolet Halos around Spiral Galaxies. I. Morphology. ApJ833(1):58, DOI 10.3847/1538-4357/833/1/58, 1610.02404

Hodges-Kluck EJ, Miller MJ, Bregman JN (2016b) The Rotation of the Hot Gas around the Milky Way. ApJ822(1):21, DOI 10.3847/0004-637X/822/1/21, 1603.07734

Hollenbach D, Salpeter EE (1971) Surface Recombination of Hydrogen Molecules. ApJ163:155, DOI $10.1086 / 150754$

Hollenbach DJ, Tielens AGGM (1999) Photodissociation regions in the interstellar medium of galaxies. Reviews of Modern Physics 71(1):173-230, DOI 10.1103/RevModPhys.71.173

Hollyhead K, Bastian N, Adamo A, Silva-Villa E, Dale J, Ryon JE, Gazak Z (2015) Studying the YMC population of M83: how long clusters remain embedded, their interaction with the ISM and implications for GC formation theories. MNRAS449(1):1106-1117, DOI 10.1093/mnras/stv331, 1502.03823

Hönig SF, Kishimoto M (2017) Dusty Winds in Active Galactic Nuclei: Reconciling Observations with Models. ApJLett838(2):L20, DOI 10.3847/2041-8213/aa6838, 1703.07781

Hönig SF, Kishimoto M, Antonucci R, Marconi A, Prieto MA, Tristram K, Weigelt G (2012) Parsec-scale Dust Emission from the Polar Region in the Type 2 Nucleus of NGC 424. ApJ755(2):149, DOI 10.1088/0004-637X/755/2/149, 1206.4307

Hönig SF, Kishimoto M, Tristram KRW, Prieto MA, Gandhi P, Asmus D, Antonucci R, Burtscher L, Duschl WJ, Weigelt G (2013) Dust in the Polar Region as a Major Contributor to the Infrared Emission of Active Galactic Nuclei. ApJ771(2):87, DOI 10.1088/0004-637X/771/2/87, 1306.4312

Hoopes CG, Sembach KR, Howk JC, Savage BD, Fullerton AW (2002) A Far Ultraviolet Spectroscopic Explorer Survey of Interstellar O VI Absorption in the Small Magellanic Cloud. ApJ569(1):233-244, DOI $10.1086 / 339323$

Hoopes CG, Heckman TM, Strickland DK, Seibert M, Madore BF, Rich RM, Bianchi L, Gil de Paz A, Burgarella D, Thilker DA, Friedman PG, Barlow TA, Byun YI, Donas J, Forster K, Jelinsky PN, Lee YW, Malina RF, Martin DC, Milliard B, Morrissey PF, Neff SG, Schiminovich D, Siegmund OHW, Small T, Szalay AS, Welsh BY, Wyder TK (2005) GALEX Observations of the Ultraviolet Halos of NGC 253 and M82. ApJLett619(1):L99-L102, DOI 10.1086/423032, astro-ph/0411309

Hopkins PF, Elvis M (2010) Quasar feedback: more bang for your buck. MNRAS401(1):7-14, DOI 10.1111/j.1365-2966.2009.15643.x, 0904.0649

Hopkins PF, Grudić MY (2019) Numerical problems in coupling photon momentum (radiation pressure) to gas. MNRAS483(3):4187-4196, DOI 10.1093/mnras/sty3089, 1803.07573

Hopkins PF, Quataert E, Murray N (2012) Stellar feedback in galaxies and the origin of galaxy-scale winds. MNRAS421(4):3522-3537, DOI 10.1111/j.1365-2966.2012.20593.x, 1110.4638

Hopkins PF, Kereš D, Oñorbe J, Faucher-Giguère CA, Quataert E, Murray N, Bullock JS (2014) Galaxies 
on FIRE (Feedback In Realistic Environments): stellar feedback explains cosmologically inefficient star formation. MNRAS445(1):581-603, DOI 10.1093/mnras/stu1738, 1311.2073

Hopkins PF, Torrey P, Faucher-Giguère CA, Quataert E, Murray N (2016) Stellar and quasar feedback in concert: effects on AGN accretion, obscuration, and outflows. MNRAS458(1):816-831, DOI 10. $1093 / \mathrm{mnras} / \mathrm{stw} 289,1504.05209$

Hopkins PF, Grudic MY, Wetzel AR, Keres D, Gaucher-Giguere CA, Ma X, Murray N, Butcher N (2018a) Radiative Stellar Feedback in Galaxy Formation: Methods and Physics. arXiv e-prints arXiv: $1811.12462,1811.12462$

Hopkins PF, Wetzel A, Kereš D, Faucher-Giguère CA, Quataert E, Boylan-Kolchin M, Murray N, Hayward CC, Garrison-Kimmel S, Hummels C, Feldmann R, Torrey P, Ma X, Anglés-Alcázar D, Su KY, Orr M, Schmitz D, Escala I, Sanderson R, Grudić MY, Hafen Z, Kim JH, Fitts A, Bullock JS, Wheeler C, Chan TK, Elbert OD, Narayanan D (2018b) FIRE-2 simulations: physics versus numerics in galaxy formation. MNRAS480(1):800-863, DOI 10.1093/mnras/sty1690, 1702.06148

Howk JC, Savage BD (1997) Extraplanar Dust in the Edge-On Spiral NGC 891. AJ114:2463, DOI 10. 1086/118660, astro-ph/9709197

Howk JC, Savage BD (1999) A Search for Extraplanar Dust in Nearby Edge-on Spirals. AJ117(5):20772101, DOI 10.1086/300857, astro-ph/9902061

Howk JC, Savage BD (2000) The Multiphase Halo of NGC 891: WIYN H $\alpha$ and BVI Imaging. AJ119(2):644-667, DOI 10.1086/301210, astro-ph/9910248

Howk JC, Sembach KR, Savage BD, Massa D, Friedman SD, Fullerton AW (2002) The Global Content, Distribution, and Kinematics of Interstellar O VI in the Large Magellanic Cloud. ApJ569(1):214-232, DOI 10.1086/339322, astro-ph/0111566

Hsieh PY, Ho PTP, Hwang CY, Shimajiri Y, Matsushita S, Koch PM, Iono D (2016) The Fossil Nuclear Outflow in the Central $30 \mathrm{pc}$ of the Galactic Center. ApJ831(1):72, DOI 10.3847/0004-637X/831/1/ $72,1607.03673$

Hu CY, Zhukovska S, Somerville RS, Naab T (2019) Thermal and non-thermal dust sputtering in hydrodynamical simulations of the multiphase interstellar medium. MNRAS487(3):3252-3269, DOI $10.1093 / \mathrm{mnras} / \mathrm{stz} 1481,1902.01368$

Huang X, Davis SW, Zhang D (2019) Dusty Cloud Acceleration with Multiband Radiation. arXiv e-prints arXiv: $1908.01775,1908.01775$

Hummels CB, Smith BD, Silvia DW (2017) Trident: A Universal Tool for Generating Synthetic Absorption Spectra from Astrophysical Simulations. ApJ847(1):59, DOI 10.3847/1538-4357/aa7e2d, 1612.03935

Humphreys RM, Davidson K, Jones TJ, Pogge RW, Grammer SH, Prieto JL, Pritchard TA (2012) The Unusual Temporal and Spectral Evolution of SN201 ht. II. Peculiar Type IIn or Impostor? ApJ760(1):93, DOI 10.1088/0004-637X/760/1/93, 1207.5755

Hutton S, Ferreras I, Wu K, Kuin P, Breeveld A, Yershov V, Cropper M, Page M (2014) A panchromatic analysis of starburst galaxy M82: probing the dust properties. MNRAS440(1):150-160, DOI 10.1093/ mnras/stu185, 1401.7669

Imanishi M, Nakanishi K, Izumi T (2016) ALMA Investigation of Vibrationally Excited $\mathrm{HCN} / \mathrm{HCO}^{+} / \mathrm{HNC}$ Emission Lines in the AGN-Hosting Ultraluminous Infrared Galaxy IRAS 20551-4250. ApJ825(1):44, DOI 10.3847/0004-637X/825/1/44, 1605.00644

Impellizzeri CMV, Gallimore JF, Baum SA, Elitzur M, Davies R, Lutz D, Maiolino R, Marconi A, Nikutta R, O'Dea CP, Sani E (2019) Counter-Rotation and High Velocity Outflow in the Parsec-Scale Molecular Torus of NGC 1068. arXiv e-prints arXiv:1908.07981, 1908.07981

Indriolo N, Geballe TR, Oka T, McCall BJ (2007) $\mathrm{H}^{+}{ }_{3}$ in Diffuse Interstellar Clouds: A Tracer for the Cosmic-Ray Ionization Rate. ApJ671(2):1736-1747, DOI 10.1086/523036, 0709.1114

Irons WT, Lacy JH, Richter MJ (2012) Ionized Gas in the Galactic Center: New Observations and Interpretation. ApJ755(2):90, DOI 10.1088/0004-637X/755/2/90, 1206.2650

Irwin JA, Madden SC (2006) Discovery of PAHs in the halo of NGC 5907. A\&A445(1):123-141, DOI 10.1051/0004-6361:20053233, astro-ph/0509726

Irwin JA, Kennedy H, Parkin T, Madden S (2007) PAHs in the halo of NGC 5529. A\&A474(2):461-472, DOI 10.1051/0004-6361:20077729, 0708.3808

Ishibashi W, Fabian AC (2012) Active galactic nucleus feedback and triggering of star formation in galaxies. MNRAS427(4):2998-3005, DOI 10.1111/j.1365-2966.2012.22074.x, 1209.1480

Ishibashi W, Fabian AC (2015) AGN feedback: galactic-scale outflows driven by radiation pressure on dust. MNRAS451(1):93-102, DOI 10.1093/mnras/stv944, 1504.07393

Ishibashi W, Fabian AC (2017) Ultramassive black hole feedback in compact galaxies. 
MNRAS472(3):2768-2772, DOI 10.1093/mnras/stx2212, 1709.01551

Ishibashi W, Fabian AC (2018) Variations on a theme of AGN-driven outflows: luminosity evolution and ambient density distribution. MNRAS481(4):4522-4531, DOI 10.1093/mnras/sty2617, 1809.07804

Ishibashi W, Fabian AC, Canning REA (2013) Can AGN feedback-driven star formation explain the size evolution of massive galaxies? MNRAS431(3):2350-2355, DOI 10.1093/mnras/stt333, 1302 . 4998

Ishibashi W, Banerji M, Fabian AC (2017) AGN radiative feedback in dusty quasar populations. MNRAS469(2):1496-1501, DOI 10.1093/mnras/stx921, 1704.03712

Ishibashi W, Fabian AC, Maiolino R (2018a) The energetics of AGN radiation pressure-driven outflows. MNRAS476(1):512-519, DOI 10.1093/mnras/sty236, 1801.09700

Ishibashi W, Fabian AC, Ricci C, Celotti A (2018b) Revisiting the 'forbidden' region: AGN radiative feedback with radiation trapping. MNRAS479(3):3335-3342, DOI 10.1093/mnras/sty1620, 1806. Q88387

Israel FP, Rosenberg MJF, van der Werf P (2015) Neutral carbon and CO in 76 (U)LIRGs and starburst galaxy centers. A method to determine molecular gas properties in luminous galaxies. A\&A578:A95, DOI 10.1051/0004-6361/201425175, 1504.08005

Janssen AW, Christopher N, Sturm E, Veilleux S, Contursi A, González-Alfonso E, Fischer J, Davies R, Verma A, Graciá-Carpio J, Genzel R, Lutz D, Sternberg A, Tacconi L, Burtscher L, Poglitsch A (2016) Broad [C II] Line Wings as Tracer of Molecular and Multi-phase Outflows in Infrared Bright Galaxies. ApJ822(1):43, DOI 10.3847/0004-637X/822/1/43, 1604.00185

Jenkins EB (2009) A Unified Representation of Gas-Phase Element Depletions in the Interstellar Medium. ApJ700(2):1299-1348, DOI 10.1088/0004-637X/700/2/1299, 0905. 3173

Jenkins EB, Tripp TM (2001) The Distribution of Thermal Pressures in the Interstellar Medium from a Survey of C I Fine-Structure Excitation. ApJSupp.137(2):297-340, DOI 10.1086/323326, astro-ph/ Q107177

Jiang YF, Stone JM, Davis SW (2014) A Global Three-dimensional Radiation Magneto-hydrodynamic Simulation of Super-Eddington Accretion Disks. ApJ796(2):106, DOI 10.1088/0004-637X/796/2/ $106,1410.0678$

Jiao Q, Zhao Y, Zhu M, Lu N, Gao Y, Zhang ZY (2017) Neutral Carbon Emission in Luminous Infrared Galaxies: The [C I] Lines as Total Molecular Gas Tracers. ApJLett840(2):L18, DOI 10.3847/2041-8213/aa6f0f, 1704.07780

Jo YS, Seon Ki, Shinn JH, Yang Y, Lee D, Min KW (2018) Comparison of the Extraplanar H $\alpha$ and UV Emissions in the Halos of Nearby Edge-on Spiral Galaxies. ApJ862(1):25, DOI 10.3847/1538-4357/ aacbca, 1806.06525

Johnson SD, Chen HW, Mulchaey JS (2015) On the origin of excess cool gas in quasar host haloes. MNRAS452(3):2553-2565, DOI 10.1093/mnras/stv1481, 1505.07838

Johnson SD, Chen HW, Mulchaey JS, Schaye J, Straka LA (2017) The Extent of Chemically Enriched Gas around Star-forming Dwarf Galaxies. ApJLett850(1):L10, DOI 10.3847/2041-8213/aa9370, 1710. 06441

Jones AP (1988) Modelling interstellar extinction. I - Porous grains. MNRAS234:209-218, DOI 10.1093/ mnras/234.2.209

Jones AP, Tielens AGGM, Hollenbach DJ, McKee CF (1994) Grain Destruction in Shocks in the Interstellar Medium. ApJ433:797, DOI 10.1086/174689

Jones GC, Maiolino R, Caselli P, Carniani S (2019a) Detection of a high-redshift molecular outflow in a primeval hyperstarburst galaxy. arXiv e-prints arXiv:1911.09967, 1911.09967

Jones T, Stark DP, Ellis RS (2018) Dust in the Wind: Composition and Kinematics of Galaxy Outflows at the Peak Epoch of Star Formation. ApJ863(2):191, DOI 10.3847/1538-4357/aad37f, 1805.01484

Jones TJ, Dowell CD, Lopez Rodriguez E, Zweibel EG, Berthoud M, Chuss DT, Goldsmith PF, Hamilton RT, Hanany S, Harper DA, Lazarian A, Looney LW, Michail JM, Morris MR, Novak G, Santos FP, Sheth K, Stacey GJ, Staguhn J, Stephens IW, Tassis K, Trinh CQ, Volpert CG, Werner M, Wollack EJ, HAWC+ Science Team (2019b) SOFIA Far-infrared Imaging Polarimetry of M82 and NGC 253: Exploring the Supergalactic Wind. ApJLett870(1):L9, DOI 10.3847/2041-8213/aaf8b9, 1812.06816

Kacprzak GG, Churchill CW, Nielsen NM (2012) Tracing Outflows and Accretion: A Bimodal Azimuthal Dependence of Mg II Absorption. ApJLett760(1):L7, DOI 10.1088/2041-8205/760/1/L7, 1205.0245

Kacprzak GG, Martin CL, Bouché N, Churchill CW, Cooke J, LeReun A, Schroetter I, Ho SH, Klimek E (2014) New Perspective on Galaxy Outflows from the First Detection of Both Intrinsic and Traverse Metal-line Absorption. ApJLett792(1):L12, DOI 10.1088/2041-8205/792/1/L12, 1407.4126

Kacprzak GG, Pointon SK, Nielsen NM, Churchill CW, Muzahid S, Charlton JC (2019) The Relationship Between Galaxy ISM and Circumgalactic Gas Metallicities. arXiv e-prints arXiv:1910.04310, 1910. 
04310

Kallivayalil N, van der Marel RP, Alcock C (2006) Is the SMC Bound to the LMC? The Hubble Space Telescope Proper Motion of the SMC. ApJ652(2):1213-1229, DOI 10.1086/508014, astro-ph/0606240

Kamenetzky J, Rangwala N, Glenn J, Maloney PR, Conley A (2016) $L_{\text {CO }}^{\prime} / L_{\text {FIR }}$ Relations with CO Rotational Ladders of Galaxies Across the Herschel SPIRE Archive. ApJ829(2):93, DOI 10.3847/ 0004-637X/829/2/93, 1508.05102

Kamenetzky J, Privon GC, Narayanan D (2018) Recovering the Physical Properties of Molecular Gas in Galaxies from CO SLED Modeling. ApJ859(1):9, DOI 10.3847/1538-4357/aab3e2, 1803.07084

Kant I (1755) Allgemeine Naturgeschichte und Theorie des Himmels

Kapińska AD, Staveley-Smith L, Crocker R, Meurer GR, Bhandari S, Hurley-Walker N, Offringa AR, Hanish DJ, Seymour N, Ekers RD, Bell ME, Callingham JR, Dwarakanath KS, For BQ, Gaensler BM, Hancock PJ, Hindson L, Johnston-Hollitt M, Lenc E, McKinley B, Morgan J, Procopio P, Wayth RB, Wu C, Zheng Q, Barry N, Beardsley AP, Bowman JD, Briggs F, Carroll P, Dillon JS, EwallWice A, Feng L, Greenhill LJ, Hazelton BJ, Hewitt JN, Jacobs DJ, Kim HS, Kittiwisit P, Line J, Loeb A, Mitchell DA, Morales MF, Neben AR, Paul S, Pindor B, Pober JC, Riding J, Sethi SK, Udaya Shankar N, Subrahmanyan R, Sullivan IS, Tegmark M, Thyagarajan N, Tingay SJ, Trott CM, Webster RL, Wyithe SB, Cappallo RJ, Deshpand e AA, Kaplan DL, Lonsdale CJ, McWhirter SR, Morgan E, Oberoi D, Ord SM, Prabu T, Srivani KS, Williams A, Williams CL (2017) Spectral Energy Distribution and Radio Halo of NGC 253 at Low Radio Frequencies. ApJ838(1):68, DOI 10.3847/ $1538-4357 /$ aa5f5d, 1702.02434

Karim MT, Fox AJ, Jenkins EB, Bordoloi R, Wakker BP, Savage BD, Lockman FJ, Crawford SM, Jorgenson RA, Bland-Hawthorn J (2018) Probing the Southern Fermi Bubble in Ultraviolet Absorption Using Distant AGNs. ApJ860(2):98, DOI 10.3847/1538-4357/aac167, 1804 . 10617

Karman W, Grillo C, Balestra I, Rosati P, Caputi KI, Di Teodoro E, Fraternali F, Gavazzi R, Mercurio A, Prochaska JX, Rodney S, Treu T (2016) Highly ionized region surrounding SN Refsdal revealed by MUSE. A\&A585:A27, DOI 10.1051/0004-6361/201527443, 1509.07515

Kassin SA, Weiner BJ, Faber SM, Koo DC, Lotz JM, Diemand J, Harker JJ, Bundy K, Metevier AJ, Phillips AC, Cooper MC, Croton DJ, Konidaris N, Noeske KG, Willmer CNA (2007) The Stellar Mass Tully-Fisher Relation to $\mathrm{z}=1.2$ from AEGIS. ApJLett660(1):L35-L38, DOI 10.1086/517932, astro-ph/0702643

Kaufman MJ, Wolfire MG, Hollenbach DJ, Luhman ML (1999) Far-Infrared and Submillimeter Emission from Galactic and Extragalactic Photodissociation Regions. ApJ527(2):795-813, DOI 10.1086/ 308102, astro-ph/9907255

Kazandjian MV, Meijerink R, Pelupessy I, Israel FP, Spaans M (2012) Diagnostics of the molecular component of photon-dominated regions with mechanical heating. A\&A542:A65, DOI 10.1051/ 0004-6361/201118641, 1203.5058

Keeney BA, Danforth CW, Stocke JT, Penton SV, Shull JM, Sembach KR (2006) Does the Milky Way Produce a Nuclear Galactic Wind? ApJ646(2):951-964, DOI 10.1086/505128, astro-ph/0604323

Keeney BA, Stocke JT, Danforth CW, Shull JM, Pratt CT, Froning CS, Green JC, Penton SV, Savage BD (2017) Characterizing the Circumgalactic Medium of Nearby Galaxies with HST/COS and HST/STIS Absorption-line Spectroscopy. II. Methods and Models. ApJSupp.230(1):6, DOI 10.3847/1538-4365/ aa6b59, 1704.00235

Keller BW, Kruijssen JMD, Wadsley JW (2019) Entropy Driven Winds: Outflows and Fountains Lifted Gently by Buoyancy. arXiv e-prints arXiv:1909.00815, 1909.00815

Kennicutt RC, Evans NJ (2012) Star Formation in the Milky Way and Nearby Galaxies. ARA\&A50:531608, DOI 10.1146/annurev-astro-081811-125610, 1204.3552

Kewley LJ, Nicholls DC, Sutherland RS (2019) Understanding Galaxy Evolution Through Emission Lines. ARA\&A57:511-570, DOI 10.1146/annurev-astro-081817-051832, 1910.09730

Kim CG, Ostriker EC (2015) Momentum Injection by Supernovae in the Interstellar Medium. ApJ802(2):99, DOI 10.1088/0004-637X/802/2/99, 1410.1537

Kim CG, Ostriker EC (2018) Numerical Simulations of Multiphase Winds and Fountains from Starforming Galactic Disks. I. Solar Neighborhood TIGRESS Model. ApJ853(2):173, DOI 10.3847/ 1538-4357/aaa5ff, 1801.03952

Kim S, Staveley-Smith L, Dopita MA, Freeman KC, Sault RJ, Kesteven MJ, McConnell D (1998) An H I Aperture Synthesis Mosaic of the Large Magellanic Cloud. ApJ503(2):674-688, DOI 10.1086/ 306030

Kim S, Dopita MA, Staveley-Smith L, Bessell MS (1999) H I Shells in the Large Magellanic Cloud. AJ118(6):2797-2823, DOI 10.1086/301116 
Kim S, Staveley-Smith L, Dopita MA, Sault RJ, Freeman KC, Lee Y, Chu YH (2003) A Neutral Hydrogen Survey of the Large Magellanic Cloud: Aperture Synthesis and Multibeam Data Combined. ApJSupp.148(2):473-486, DOI 10.1086/376980

Kimm T, Cen R, Devriendt J, Dubois Y, Slyz A (2015) Towards simulating star formation in turbulent highz galaxies with mechanical supernova feedback. MNRAS451(3):2900-2921, DOI 10.1093/mnras/ stv1211, 1501.05655

Kimm T, Blaizot J, Garel T, Michel-Dansac L, Katz H, Rosdahl J, Verhamme A, Haehnelt M (2019) Understanding the escape of LyC and Ly $\alpha$ photons from turbulent clouds. MNRAS486(2):2215-2237, DOI 10.1093/mnras/stz989, 1901.05990

Kimura H, Mann I, Biesecker DA, Jessberger EK (2002) Dust Grains in the Comae and Tails of Sungrazing Comets: Modeling of Their Mineralogical and Morphological Properties. Icarus 159(2):529-541, DOI 10.1006/icar.2002.6940

King A (2003) Black Holes, Galaxy Formation, and the $\mathbf{M}_{B H^{-}} \sigma$ Relation. ApJLett596(1):L27-L29, DOI $10.1086 / 379143$, astro-ph/0308342

King A, Pounds K (2015) Powerful Outflows and Feedback from Active Galactic Nuclei. ARA\&A53:115154, DOI 10.1146/annurev-astro-082214-122316, 1503.05206

King AR (2010) Black hole outflows. MNRAS402(3):1516-1522, DOI 10.1111/j.1365-2966.2009.16013. $\mathrm{X}, 0911.1639$

Klein RI, McKee CF, Colella P (1994) On the Hydrodynamic Interaction of Shock Waves with Interstellar Clouds. I. Nonradiative Shocks in Small Clouds. ApJ420:213, DOI 10.1086/173554

Kochanek CS (2011) The Astrophysical Implications of Dust Formation during the Eruptions of Hot, Massive Stars. ApJ743(1):73, DOI 10.1088/0004-637X/743/1/73, 1109.2596

Koratkar A, Blaes O (1999) The Ultraviolet and Optical Continuum Emission in Active Galactic Nuclei: The Status of Accretion Disks. PASP111(755):1-30, DOI 10.1086/316294

Kormendy J, Ho LC (2013) Coevolution (Or Not) of Supermassive Black Holes and Host Galaxies. ARA\&A51(1):511-653, DOI 10.1146/annurev-astro-082708-101811, 1304.7762

Kornei KA, Shapley AE, Martin CL, Coil AL, Lotz JM, Schiminovich D, Bundy K, Noeske KG (2012) The Properties and Prevalence of Galactic Outflows at $z \sim 1$ in the Extended Groth Strip. ApJ758(2):135, DOI 10.1088/0004-637X/758/2/135, 1205.0812

Kornei KA, Shapley AE, Martin CL, Coil AL, Lotz JM, Weiner BJ (2013) Fine-structure Fe II* Emission and Resonant Mg II Emission in $z \sim 1$ Star-forming Galaxies. ApJ774(1):50, DOI 10.1088/0004-637X/774/1/50, 1302.6997

Koudmani S, Sijacki D, Bourne MA, Smith MC (2019) Fast and energetic AGN-driven outflows in simulated dwarf galaxies. MNRAS484(2):2047-2066, DOI 10.1093/mnras/stz097, 1812.04629

Koyama K, Maeda Y, Sonobe T, Takeshima T, Tanaka Y, Yamauchi S (1996) ASCA View of Our Galactic Center: Remains of Past Activities in X-Rays? PASJ48:249-255, DOI 10.1093/pasj/48.2.249

Kreckel K, Armus L, Groves B, Lyubenova M, Díaz-Santos T, Schinnerer E, Appleton P, Croxall KV, Dale DA, Hunt LK, Beirão P, Bolatto AD, Calzetti D, Donovan Meyer J, Draine BT, Hinz J, Kennicutt RC, Meidt S, Murphy EJ, Smith JDT, Tabatabaei FS, Walter F (2014) A Far-IR View of the Starburstdriven Superwind in NGC 2146. ApJ790(1):26, DOI 10.1088/0004-637X/790/1/26, 1403. 2381

Krieger N, Bolatto AD, Walter F, Leroy AK, Zschaechner LK, Meier DS, Ott J, Weiss A, Mills EAC, Levy RC, Veilleux S, Gorski M (2019) The Molecular Outflow in NGC 253 at a Resolution of Two Parsecs. ApJ881(1):43, DOI 10.3847/1538-4357/ab2d9c, 1907.00731

Krips M, Martín S, Eckart A, Neri R, García-Burillo S, Matsushita S, Peck A, Stoklasová I, Petitpas G, Usero A, Combes F, Schinnerer E, Humphreys E, Baker AJ (2011) Submillimeter Array/Plateau de Bure Interferometer Multiple Line Observations of the Nearby Seyfert 2 Galaxy NGC 1068: Shockrelated Gas Kinematics and Heating in the Central 100 pc? ApJ736(1):37, DOI 10.1088/0004-637X/ $736 / 1 / 37,1105.6089$

Krug HB, Rupke DSN, Veilleux S (2010) Neutral Gas Outflows and Inflows in Infrared-faint Seyfert Galaxies. ApJ708(2):1145-1161, DOI 10.1088/0004-637X/708/2/1145, 0911. 3897

Kruijssen JMD, Longmore SN (2014) An uncertainty principle for star formation - I. Why galactic star formation relations break down below a certain spatial scale. MNRAS439(4):3239-3252, DOI 10 1093/mnras/stu098, 1401.4459

Kruijssen JMD, Dale JE, Longmore SN (2015) The dynamical evolution of molecular clouds near the Galactic Centre - I. Orbital structure and evolutionary timeline. MNRAS447(2):1059-1079, DOI $10.1093 / \mathrm{mnras} / \mathrm{stu} 2526,1412.0664$

Kruijssen JMD, Schruba A, Chevance M, Longmore SN, Hygate AePS, Haydon DT, McLeod AF, Dalcanton JJ, Tacconi LJ, van Dishoeck EF (2019) Fast and inefficient star formation due to short-lived 
molecular clouds and rapid feedback. Nature569(7757):519-522, DOI 10.1038/s41586-019-1194-3, 1905.08801

Krumholz MR (2018) Resolution requirements and resolution problems in simulations of radiative feedback in dusty gas. MNRAS480(3):3468-3482, DOI 10.1093/mnras/sty2105, 1805.00210

Krumholz MR, Thompson TA (2013) Numerical simulations of radiatively driven dusty winds. MNRAS434(3):2329-2346, DOI 10.1093/mnras/stt1174, 1302.4440

Krumholz MR, Kruijssen JMD, Crocker RM (2017a) A dynamical model for gas flows, star formation and nuclear winds in galactic centres. MNRAS466(1):1213-1233, DOI 10.1093/mnras/stw3195, 1605. 02850

Krumholz MR, Thompson TA, Ostriker EC, Martin CL (2017b) The observable properties of cool winds from galaxies, AGN, and star clusters - I. Theoretical framework. MNRAS471(4):4061-4086, DOI $10.1093 / \mathrm{mnras} / \mathrm{stx} 1882,1702.05102$

Krumholz MR, McKee CF, Bland-Hawthorn J (2019) Star Clusters Across Cosmic Time. ARA\&A57:227303, DOI 10.1146/annurev-astro-091918-104430, 1812.01615

Kulkarni VP, Som D, Morrison S, Péroux C, Quiret S, York DG (2015) Keck and VLT Observations of Super-Damped Lyman-Alpha Absorbers at $z \sim 2-2.5$ : Constraints on Chemical Compositions and Physical Conditions. ApJ815(1):24, DOI 10.1088/0004-637X/815/1/24, 1510.05342

Lacy JH, Achtermann JM, Serabyn E (1991) Galactic Center Gasdynamics: A One-armed Spiral in a Keplerian Disk. ApJLett380:L71, DOI 10.1086/186176

Lakićević M, van Loon JT, Meixner M, Gordon K, Bot C, Roman-Duval J, Babler B, Bolatto A, Engelbracht C, Filipović M, Hony S, Indebetouw R, Misselt K, Montiel E, Okumura K, Panuzzo P, Patat F, Sauvage M, Seale J, Sonneborn G, Temim T, Urošević D, Zanardo G (2015) The Influence of Supernova Remnants on the Interstellar Medium in the Large Magellanic Cloud Seen at 20-600 $\mu \mathrm{m}$ Wavelengths. ApJ799(1):50, DOI 10.1088/0004-637X/799/1/50, 1410.5709

Lan TW (2019) The Co-Evolution of Galaxies and the Cool Circumgalactic Medium Probed with the SDSS and DESI Legacy Imaging Surveys. arXiv e-prints arXiv:1911.01271, 1911.01271

Lan TW, Mo H (2018) The Circumgalactic Medium of eBOSS Emission Line Galaxies: Signatures of Galactic Outflows in Gas Distribution and Kinematics. ApJ866(1):36, DOI 10.3847/1538-4357/ aadc08, 1806.05786

Lan TW, Ménard B, Zhu G (2014) The Properties of the Cool Circumgalactic Gas Probed with the SDSS, WISE, and GALEX Surveys. ApJ795(1):31, DOI 10.1088/0004-637X/795/1/31, 1404.5301

Langer WD, Pineda JL (2015) [C ii] emission from galactic nuclei in the presence of X-rays. A\&A580:A5, DOI 10.1051/0004-6361/201525950, 1505.02777

Lau MW, Prochaska JX, Hennawi JF (2016) Quasars Probing Quasars. VIII. The Physical Properties of the Cool Circumgalactic Medium Surrounding $z \sim 2-3$ Massive Galaxies Hosting Quasars. ApJSupp.226(2):25, DOI 10.3847/0067-0049/226/2/25, 1510.06018

Lau MW, Prochaska JX, Hennawi JF (2018) Quasars Probing Quasars. IX. The Kinematics of the Circumgalactic Medium Surrounding $z \sim 2$ Quasars. ApJ857(2):126, DOI 10.3847/1538-4357/aab78e, 1705.03476

Laursen P, Sommer-Larsen J, Andersen AC (2009) Ly $\alpha$ Radiative Transfer with Dust: Escape Fractions from Simulated High-Redshift Galaxies. ApJ704(2):1640-1656, DOI 10.1088/0004-637X/704/ 2/1640, 0907.2698

Le Bourlot J, Le Petit F, Pinto C, Roueff E, Roy F (2012) Surface chemistry in the interstellar medium. I. $\mathrm{H}_{2}$ formation by Langmuir-Hinshelwood and Eley-Rideal mechanisms. A\&A541:A76, DOI 10.1051/ 0004-6361/201118126, 1202.0374

Leaman R, Fragkoudi F, Querejeta M, Leung GYC, Gadotti DA, Husemann B, Falcón-Barroso J, Sánchez-Blázquez P, van de Ven G, Kim T, Coelho P, Lyubenova M, de Lorenzo-Cáceres A, Martig M, Martinez-Valpuesta I, Neumann J, Pérez I, Seidel M (2019) Survival of molecular gas in a stellar feedback-driven outflow witnessed with the MUSE TIMER project and ALMA. MNRAS488(3):3904-3928, DOI 10.1093/mnras/stz1844, 1907.13142

Leftley JH, Hönig SF, Asmus D, Tristram KRW, Gandhi P, Kishimoto M, Venanzi M, Williamson DJ (2019) Parsec-scale Dusty Winds in Active Galactic Nuclei: Evidence for Radiation Pressure Driving. arXiv e-prints arXiv: $1910.00600,1910.00600$

Lehner N, Howk JC (2007) Highly ionized plasma in the Large Magellanic Cloud: evidence for outflows and a possible galactic wind. MNRAS377(2):687-704, DOI 10.1111/j.1365-2966.2007.11631.x, astro-ph/0702427

Lehner N, Staveley-Smith L, Howk JC (2009) Properties and Origin of the High-Velocity Gas Toward the Large Magellanic Cloud. ApJ702(2):940-954, DOI 10.1088/0004-637X/702/2/940, 0907.2416 
Lehner N, Howk JC, Tripp TM, Tumlinson J, Prochaska JX, O’Meara JM, Thom C, Werk JK, Fox AJ, Ribaudo J (2013) The Bimodal Metallicity Distribution of the Cool Circumgalactic Medium at $z \lesssim 1$. ApJ770(2):138, DOI 10.1088/0004-637X/770/2/138, 1302.5424

Lehner N, Wotta CB, Howk JC, O’Meara JM, Oppenheimer BD, Cooksey KL (2019) The COS CGM Compendium. III: Metallicity and Physical Properties of the Cool Circumgalactic Medium at z\&lt;1. arXiv e-prints arXiv:1902.10147, 1902 .10147

Lehnert MD, Heckman TM, Weaver KA (1999) Very Extended X-Ray and H $\alpha$ Emission in M82: Implications for the Superwind Phenomenon. ApJ523(2):575-584, DOI 10.1086/307762, astro-ph/ 9904227

Leibler CN, Cantalupo S, Holden BP, Madau P (2018) The detection of intergalactic $\mathrm{H} \alpha$ emission from the Slug Nebula at $z \sim 2.3$. MNRAS480(2):2094-2108, DOI 10.1093/mnras/sty1764, 1807.00054

Leighly KM, Terndrup DM, Baron E, Lucy AB, Dietrich M, Gallagher SC (2014) Evidence for Active Galactic Nucleus Feedback in the Broad Absorption Lines and Reddening of Mrk 231. ApJ788(2):123, DOI 10.1088/0004-637X/788/2/123, 1404.6795

Leitherer C, Schaerer D, Goldader JD, Delgado RMG, Robert C, Kune DF, de Mello DF, Devost D, Heckman TM (1999) Starburst99: Synthesis Models for Galaxies with Active Star Formation. ApJSupp.123(1):3-40, DOI 10.1086/313233, astro-ph/9902334

Leitherer C, Chandar R, Tremonti CA, Wofford A, Schaerer D (2013) Far-ultraviolet Observations of Outflows from Infrared-luminous Galaxies. ApJ772(2):120, DOI 10.1088/0004-637X/772/2/120, 1306.0419

Leroy AK, Bolatto AD, Ostriker EC, Rosolowsky E, Walter F, Warren SR, Donovan Meyer J, Hodge J, Meier DS, Ott J, Sandstrom K, Schruba A, Veilleux S, Zwaan M (2015a) ALMA Reveals the Molecular Medium Fueling the Nearest Nuclear Starburst. ApJ801(1):25, DOI 10.1088/0004-637X/ $801 / 1 / 25,1411.2836$

Leroy AK, Walter F, Martini P, Roussel H, Sandstrom K, Ott J, Weiss A, Bolatto AD, Schuster K, Dessauges-Zavadsky M (2015b) The Multi-phase Cold Fountain in M82 Revealed by a Wide, Sensitive Map of the Molecular Interstellar Medium. ApJ814(2):83, DOI 10.1088/0004-637X/814/2/83, 1509.02932

Leroy AK, Usero A, Schruba A, Bigiel F, Kruijssen JMD, Kepley A, Blanc GA, Bolatto AD, Cormier D, Gallagher M, Hughes A, Jiménez-Donaire MJ, Rosolowsky E, Schinnerer E (2017) Millimeter-wave Line Ratios and Sub-beam Volume Density Distributions. ApJ835(2):217, DOI 10.3847/1538-4357/ $835 / 2 / 217,1611.09864$

Leroy AK, Bolatto AD, Ostriker EC, Walter F, Gorski M, Ginsburg A, Krieger N, Levy RC, Meier DS, Mills E, Ott J, Rosolowsky E, Thompson TA, Veilleux S, Zschaechner LK (2018) Forming Super Star Clusters in the Central Starburst of NGC 253. ApJ869(2):126, DOI 10.3847/1538-4357/aaecd1, 1804.02083

Li A, Draine BT (2001) Infrared Emission from Interstellar Dust. II. The Diffuse Interstellar Medium. ApJ554(2):778-802, DOI 10.1086/323147, astro-ph/0011319

Li JT, Bregman JN, Wang QD, Crain RA, Anderson ME (2018) Baryon Budget of the Hot Circumgalactic Medium of Massive Spiral Galaxies. ApJLett855(2):L24, DOI 10.3847/2041-8213/aab2af, 1802. 09453

Li Z, Hopkins PF, Squire J, Hummels C (2019) On the Survival of Cool Clouds in the Circum-Galactic Medium. arXiv e-prints arXiv:1909.02632, 1909.02632

Lindberg JE, Aalto S, Muller S, Martí-Vidal I, Falstad N, Costagliola F, Henkel C, van der Werf P, GarcíaBurillo S, González-Alfonso E (2016) Evidence for a chemically differentiated outflow in Mrk 231. A\&A587:A15, DOI 10.1051/0004-6361/201527457, 1512.04854

Liszt H (2001) CO J = 3-2 mapping of the molecular circumnuclear disk in Centaurus A. A\&A371:865874, DOI 10.1051/0004-6361:20010443

Liu RY, Murase K, Inoue S, Ge C, Wang XY (2018) Can Winds Driven by Active Galactic Nuclei Account for the Extragalactic Gamma-Ray and Neutrino Backgrounds? ApJ858(1):9, DOI 10.3847/1538-4357/aaba74, 1712.10168

Lockman FJ (1984) The HI halo in the inner galaxy. ApJ283:90-97, DOI 10.1086/162277

Lockman FJ, McClure-Griffiths NM (2016) Tracing the Milky Way Nuclear Wind with $21 \mathrm{~cm}$ Atomic Hydrogen Emission. ApJ826(2):215, DOI 10.3847/0004-637X/826/2/215, 1605.01140

Lockman FJ, Di Teodoro EM, McClure-Griffiths NM (2019) Observation of Acceleration of HI Clouds Within the Fermi Bubbles. arXiv e-prints arXiv:1911.06864, 1911.06864

Longmore SN, Kruijssen JMD, Bastian N, Bally J, Rathborne J, Testi L, Stolte A, Dale J, Bressert E, Alves J (2014) The Formation and Early Evolution of Young Massive Clusters. In: Beuther H, Klessen 
RS, Dullemond CP, Henning T (eds) Protostars and Planets VI, p 291, DOI 10.2458/azu_uapress 9780816531240-ch013, 1401.4175

López-Gonzaga N, Jaffe W, Burtscher L, Tristram KRW, Meisenheimer K (2014) Revealing the large nuclear dust structures in NGC 1068 with MIDI/VLTI. A\&A565:A71, DOI 10.1051/0004-6361/ 201323002, 1401.3248

López-Gonzaga N, Burtscher L, Tristram KRW, Meisenheimer K, Schartmann M (2016) Mid-infrared interferometry of 23 AGN tori: On the significance of polar-elongated emission. A\&A591:A47, DOI 10.1051/0004-6361/201527590, 1602.05592

Lu JR, Do T, Ghez AM, Morris MR, Yelda S, Matthews K (2013) Stellar Populations in the Central 0.5 pc of the Galaxy. II. The Initial Mass Function. ApJ764(2):155, DOI 10.1088/0004-637X/764/2/155, 1301.0540

Lutz D, Sturm E, Janssen A, Veilleux S, Aalto S, Cicone C, Contursi A, Davies RI, Feruglio C, Fischer J, Garcia-Burillo S, Genzel R, González-Alfonso E, Herrera-Camus JGCR, Maiolino R, Schruba A, Shimizu T, Sternberg A, Tacconi LJ, Weiß A (2019) Molecular outflows in local galaxies: Method comparison and a role of intermittent AGN driving. arXiv e-prints arXiv:1911.05608, 1911.05608

Lynden-Bell D (2003) On why discs generate magnetic towers and collimate jets. MNRAS341(4):13601372, DOI 10.1046/j.1365-8711.2003.06506.x, astro-ph/0208388

Lynden-Bell D (2006) Magnetic jets from swirling discs. MNRAS369(3):1167-1188, DOI 10.1111/j. 1365-2966.2006.10349.x, astro-ph/0604424

Ma J, Ge J, Zhao Y, Prochaska JX, Zhang S, Ji T, Schneider DP (2017) Quasar 2175 Å dust absorbers - I. Metallicity, depletion pattern and kinematics. MNRAS472(2):2196-2220, DOI 10.1093/mnras/ stx2117, 1708.04200

Mac Low MM, Ferrara A (1999) Starburst-driven Mass Loss from Dwarf Galaxies: Efficiency and Metal Ejection. ApJ513(1):142-155, DOI 10.1086/306832, astro-ph/9801237

Magorrian J, Tremaine S, Richstone D, Bender R, Bower G, Dressler A, Faber SM, Gebhardt K, Green R, Grillmair C, Kormendy J, Lauer T (1998) The Demography of Massive Dark Objects in Galaxy Centers. AJ115(6):2285-2305, DOI 10.1086/300353, astro-ph/9708072

Maiolino R, Mannucci F (2019) De re metallica: the cosmic chemical evolution of galaxies. A\&A Rev.27(1):3, DOI 10.1007/s00159-018-0112-2, 1811.09642

Maiolino R, Schneider R, Oliva E, Bianchi S, Ferrara A, Mannucci F, Pedani M, Roca Sogorb M (2004) A supernova origin for dust in a high-redshift quasar. Nature431(7008):533-535, DOI 10.1038/nature02930, astro-ph/0409577

Maiolino R, Gallerani S, Neri R, Cicone C, Ferrara A, Genzel R, Lutz D, Sturm E, Tacconi LJ, Walter F, Feruglio C, Fiore F, Piconcelli E (2012) Evidence of strong quasar feedback in the early Universe. MNRAS425(1):L66-L70, DOI 10.1111/j.1745-3933.2012.01303.x, 1204.2904

Maiolino R, Russell HR, Fabian AC, Carniani S, Gallagher R, Cazzoli S, Arribas S, Belfiore F, Bellocchi E, Colina L, Cresci G, Ishibashi W, Marconi A, Mannucci F, Oliva E, Sturm E (2017) Star formation inside a galactic outflow. Nature544(7649):202-206, DOI 10.1038/nature21677, 1703.08587

Maltby DT, Almaini O, McLure RJ, Wild V, Dunlop J, Rowlands K, Hartley WG, Hatch NA, Socolovsky M, Wilkinson A, Amorin R, Bradshaw EJ, Carnall AC, Castellano M, Cimatti A, Cresci G, Cullen F, De Barros S, Fontanot F, Garilli B, Koekemoer AM, McLeod DJ, Pentericci L, Talia M (2019) High-velocity outflows in massive post-starburst galaxies at z \&gt; 1. MNRASp 2140, DOI 10.1093/ mnras/stz2211

Mangum JG, Shirley YL (2015) How to Calculate Molecular Column Density. PASP127(949):266, DOI $10.1086 / 680323,1501.01703$

Mangum JG, Wootten A (1993) Formaldehyde as a Probe of Physical Conditions in Dense Molecular Clouds. ApJSupp.89:123, DOI 10.1086/191841

Mangum JG, Darling J, Henkel C, Menten KM, MacGregor M, Svoboda BE, Schinnerer E (2013) Ammonia Thermometry of Star-Forming Galaxies. ApJ779(1):33, DOI 10.1088/0004-637X/779/1/33, 1310.6586

Marcolini A, Strickland DK, D'Ercole A, Heckman TM, Hoopes CG (2005) The dynamics and high-energy emission of conductive gas clouds in supernova-driven galactic superwinds. MNRAS362(2):626-648, DOI 10.1111/j.1365-2966.2005.09343.x, astro-ph/0506645

Marinacci F, Binney J, Fraternali F, Nipoti C, Ciotti L, Londrillo P (2010) The mode of gas accretion on to star-forming galaxies. MNRAS404(3):1464-1474, DOI 10.1111/j.1365-2966.2010.16352.x, 1001. 2446

Martí-Vidal I, Vlemmings WHT, Muller S, Casey S (2014) UVMULTIFIT: A versatile tool for fitting astronomical radio interferometric data. A\&A563:A136, DOI 10.1051/0004-6361/201322633, 1401. 
4984

Martin CL (1999) Properties of Galactic Outflows: Measurements of the Feedback from Star Formation. ApJ513(1):156-160, DOI 10.1086/306863, astro-ph/9810233

Martin CL (2005) Mapping Large-Scale Gaseous Outflows in Ultraluminous Galaxies with Keck II ESI Spectra: Variations in Outflow Velocity with Galactic Mass. ApJ621(1):227-245, DOI 10.1086/ 427277, astro-ph/0410247

Martin CL (2006) Mapping Large-Scale Gaseous Outflows in Ultraluminous Infrared Galaxies with Keck II ESI Spectra: Spatial Extent of the Outflow. ApJ647(1):222-243, DOI 10.1086/504886, astro-ph/ 0604173

Martin CL, Shapley AE, Coil AL, Kornei KA, Bundy K, Weiner BJ, Noeske KG, Schiminovich D (2012) Demographics and Physical Properties of Gas Outflows/Inflows at 0.4 \&lt; z \&lt; 1.4. ApJ760(2):127, DOI 10.1088/0004-637X/760/2/127, 1206. 5552

Martin CL, Shapley AE, Coil AL, Kornei KA, Murray N, Pancoast A (2013) Scattered Emission from $z \sim 1$ Galactic Outflows. ApJ770(1):41, DOI 10.1088/0004-637X/770/1/41, 1304.6405

Martin CL, Dijkstra M, Henry A, Soto KT, Danforth CW, Wong J (2015) The Ly $\alpha$ Line Profiles of Ultraluminous Infrared Galaxies: Fast Winds and Lyman Continuum Leakage. ApJ803(1):6, DOI 10.1088/0004-637X/803/1/6, 1501.05946

Martin CL, Ho SH, Kacprzak GG, Churchill CW (2019) Kinematics of Circumgalactic Gas: Feeding Galaxies and Feedback. ApJ878(2):84, DOI 10.3847/1538-4357/ab18ac, 1901.09123

Martin PG, Adamson AJ, Whittet DCB, Hough JH, Bailey JA, Kim SH, Sato S, Tamura M, Yamashita T (1992) Interstellar Polarization from 3 to 5 Microns in Reddened Stars. ApJ392:691, DOI 10.1086/ 171470

Martín S, Martín-Pintado J, Viti S (2009) Photodissociation Chemistry Footprints in the Starburst Galaxy NGC 253. ApJ706(2):1323-1330, DOI 10.1088/0004-637X/706/2/1323, 0911.2673

Martín S, Aalto S, Sakamoto K, González-Alfonso E, Muller S, Henkel C, García-Burillo S, Aladro R, Costagliola F, Harada N, Krips M, Martín-Pintado J, Mühle S, van der Werf P, Viti S (2016) The unbearable opaqueness of Arp220. A\&A590:A25, DOI 10.1051/0004-6361/201528064, 1603.01291

Martínez-González S, Wünsch R, Silich S, Tenorio-Tagle G, Palouš J, Ferrara A (2019) Supernovae within Pre-existing Wind-Blown Bubbles: Dust Injection vs. Ambient Dust Destruction. arXiv eprints arXiv: $1911.05079,1911.05079$

Martini P, Leroy AK, Mangum JG, Bolatto A, Keating KM, Sandstrom K, Walter F (2018) H I Kinematics along the Minor Axis of M82. ApJ856(1):61, DOI 10.3847/1538-4357/aab08e, 1802.04359

Martizzi D, Faucher-Giguère CA, Quataert E (2015) Supernova feedback in an inhomogeneous interstellar medium. MNRAS450(1):504-522, DOI 10.1093/mnras/stv562, 1409.4425

Mathes NL, Churchill CW, Kacprzak GG, Nielsen NM, Trujillo-Gomez S, Charlton J, Muzahid S (2014) Halo Mass Dependence of H I and O VI Absorption: Evidence for Differential Kinematics. ApJ792(2):128, DOI 10.1088/0004-637X/792/2/128, 1406.2314

Matsubayashi K, Sugai H, Shimono A, Hattori T, Ozaki S, Yoshikawa T, Taniguchi Y, Nagao T, Kajisawa M, Shioya Y, Bland-Hawthorn J (2012) Ionization Source of a Minor-axis Cloud in the Outer Halo of M82. ApJ761(1):55, DOI 10.1088/0004-637X/761/1/55, 1210.8149

Matsuura M, De Buizer JM, Arendt RG, Dwek E, Barlow MJ, Bevan A, Cigan P, Gomez HL, Rho J, Wesson R, Bouchet P, Danziger J, Meixner M (2019) SOFIA mid-infrared observations of Supernova 1987A in 2016 - forward shocks and possible dust re-formation in the post-shocked region. MNRAS482(2):1715-1723, DOI 10.1093/mnras/sty2734, 1810.03615

McCarthy PJ (1993) High redshift radio galaxies. ARA\&A31:639-688, DOI 10.1146/annurev.aa.31. 090193.003231

McClure-Griffiths NM, Green JA, Hill AS, Lockman FJ, Dickey JM, Gaensler BM, Green AJ (2013) Atomic Hydrogen in a Galactic Center Outflow. ApJLett770(1):L4, DOI 10.1088/2041-8205/770/1/ L4, 1304.7538

McClure-Griffiths NM, Dénes H, Dickey JM, Stanimirović S, Staveley-Smith L, Jameson K, Di Teodoro E, Allison JR, Collier JD, Chippendale AP, Franzen T, Gürkan G, Heald G, Hotan A, Kleiner D, LeeWaddell K, McConnell D, Popping A, Rhee J, Riseley CJ, Voronkov MA, Whiting M (2018) Cold gas outflows from the Small Magellanic Cloud traced with ASKAP. Nature Astronomy 2:901-906, DOI 10.1038/s41550-018-0608-8, 1811.01772

McCormick A, Veilleux S, Rupke DSN (2013) Dusty Winds: Extraplanar Polycyclic Aromatic Hydrocarbon Features of Nearby Galaxies. ApJ774(2):126, DOI 10.1088/0004-637X/774/2/126

McCormick A, Veilleux S, Meléndez M, Martin CL, Bland -Hawthorn J, Cecil G, Heitsch F, Müller T, Rupke DSN, Engelbracht C (2018) Exploring the dust content of galactic winds with Herschel - II. 
Nearby dwarf galaxies. MNRAS477(1):699-726, DOI 10.1093/mnras/sty634, 1803.03269

McCourt M, O'Leary RM, Madigan AM, Quataert E (2015) Magnetized gas clouds can survive acceleration by a hot wind. MNRAS449(1):2-7, DOI 10.1093/mnras/stv355, 1409.6719

McCourt M, Oh SP, O'Leary R, Madigan AM (2018) A characteristic scale for cold gas. MNRAS473(4):5407-5431, DOI 10.1093/mnras/stx2687, 1610.01164

McKinley B, Tingay SJ, Carretti E, Ellis S, Bland-Hawthorn J, Morganti R, Line J, McDonald M, Veilleux S, Wahl Olsen R, Sidonio M, Ekers R, Offringa AR, Procopio P, Pindor B, Wayth RB, Hurley-Walker N, Bernardi G, Gaensler BM, Haverkorn M, Kesteven M, Poppi S, Staveley-Smith L (2018) The jet/wind outflow in Centaurus A: a local laboratory for AGN feedback. MNRAS474(3):4056-4072, DOI 10.1093/mnras/stx2890, 1711.01751

McKinney JC, Tchekhovskoy A, Sadowski A, Narayan R (2014) Three-dimensional general relativistic radiation magnetohydrodynamical simulation of super-Eddington accretion, using a new code HARMRAD with M1 closure. MNRAS441(4):3177-3208, DOI 10.1093/mnras/stu762, 1312.6127

McKinney JC, Dai L, Avara MJ (2015) Efficiency of super-Eddington magnetically-arrested accretion. MNRAS454(1):L6-L10, DOI 10.1093/mnrasl/slv115, 1508.02433

McQuinn M, Werk JK (2018) Implications of the Large O VI Columns around Low-redshift L * Galaxies. ApJ852(1):33, DOI 10.3847/1538-4357/aa9d3f, 1703.03422

Mehdipour M, Costantini E (2018) Probing the nature and origin of dust in the reddened quasar IC 4329A with global modelling from X-ray to infrared. A\&A619:A20, DOI 10.1051/0004-6361/201833706, 1808.04628

Meier DS, Turner JL (2012) Spatially Resolved Chemistry in nearby Galaxies. II. The Nuclear Bar in Maffei 2. ApJ755(2):104, DOI 10.1088/0004-637X/755/2/104, 1206.4098

Meijerink R, Spaans M, Loenen AF, van der Werf PP (2011) Star formation in extreme environments: the effects of cosmic rays and mechanical heating. A\&A525:A119, DOI 10.1051/0004-6361/201015136, 1010.0788

Meijerink R, Kristensen LE, Weiß A, van der Werf PP, Walter F, Spaans M, Loenen AF, Fischer J, Israel FP, Isaak K, Papadopoulos PP, Aalto S, Armus L, Charmand aris V, Dasyra KM, Diaz-Santos T, Evans A, Gao Y, González-Alfonso E, Güsten R, Henkel C, Kramer C, Lord S, Martín-Pintado J, Naylor D, Sanders DB, Smith H, Spinoglio L, Stacey G, Veilleux S, Wiedner MC (2013) Evidence for CO Shock Excitation in NGC 6240 from Herschel SPIRE Spectroscopy. ApJLett762(2):L16, DOI 10.1088/2041-8205/762/2/L16, 1211.6659

Meiring JD, Kulkarni VP, Lauroesch JT, Péroux C, Khare P, York DG (2009a) New Magellan Inamori Kyocera Echelle Observations of z \&lt; 1.5 sub-damped Lyman $\alpha$ systems. MNRAS393(467):15131530, DOI 10.1111/j.1365-2966.2008.14253.x, 0902.2022

Meiring JD, Lauroesch JT, Kulkarni VP, Péroux C, Khare P, York DG (2009b) A MIKE + UVES survey of sub-damped Lyman $\alpha$ systems at z \&lt; 1.5. MNRAS397(4):2037-2048, DOI 10.1111/j.1365-2966. 2009.15064.x, 0905.4473

Meléndez M, Veilleux S, Martin C, Engelbracht C, Bland-Hawthorn J, Cecil G, Heitsch F, McCormick A, Müller T, Rupke D, Teng SH (2015) Exploring the Dust Content of Galactic Winds with Herschel. I. NGC 4631. ApJ804(1):46, DOI 10.1088/0004-637X/804/1/46, 1502.07785

Ménard B, Fukugita M (2012) Cosmic Dust in Mg II Absorbers. ApJ754(2):116, DOI 10.1088/ 0004-637X/754/2/116, 1204.1978

Ménard B, Scranton R, Fukugita M, Richards G (2010) Measuring the galaxy-mass and galaxy-dust correlations through magnification and reddening. MNRAS405(2):1025-1039, DOI 10.1111/j.1365-2966. 2010.16486.x, 0902.4240

Ménard B, Wild V, Nestor D, Quider A, Zibetti S, Rao S, Turnshek D (2011) Probing star formation across cosmic time with absorption-line systems. MNRAS417(2):801-811, DOI 10.1111/j.1365-2966.2011. 18227.x, 0912. 3263

Michiyama T, Iono D, Sliwa K, Bolatto A, Nakanishi K, Ueda J, Saito T, Ando M, Yamashita T, Yun $\mathrm{M}$ (2018) ALMA Observations of $\mathrm{HCN}$ and $\mathrm{HCO}^{+}$Outflows in the Merging Galaxy NGC 3256 . ApJ868(2):95, DOI 10.3847/1538-4357/aae82a, 1810.04821

Miller MJ, Bregman JN (2016) The Interaction of the Fermi Bubbles with the Milky Way's Hot Gas Halo. ApJ829(1):9, DOI 10.3847/0004-637X/829/1/9, 1607.04906

Miller TR, Arav N, Xu X, Kriss GA, Plesha RJ, Benn C, Liu G (2018) Distance, Energy, and Variability of Quasar Outflows: Two HST/COS Epochs of LBQS 1206+1052. ApJ865(2):90, DOI 10.3847/1538-4357/aad817, 1809.03114

Min M, Waters LBFM, de Koter A, Hovenier JW, Keller LP, Markwick-Kemper F (2007) The shape and composition of interstellar silicate grains. A\&A462(2):667-676, DOI 10.1051/0004-6361:20065436, 
astro-ph/0611329

Mingozzi M, Cresci G, Venturi G, Marconi A, Mannucci F, Perna M, Belfiore F, Carniani S, Balmaverde B, Brusa M, Cicone C, Feruglio C, Gallazzi A, Mainieri V, Maiolino R, Nagao T, Nardini E, Sani E, Tozzi P, Zibetti S (2019) The MAGNUM survey: different gas properties in the outflowing and disc components in nearby active galaxies with MUSE. A\&A622:A146, DOI 10.1051/0004-6361/ 201834372, 1811.07935

Mitra S, Davé R, Finlator K (2015) Equilibrium model constraints on baryon cycling across cosmic time. MNRAS452(2):1184-1200, DOI 10.1093/mnras/stv1387, 1411.1157

Moe M, Arav N, Bautista MA, Korista KT (2009) Quasar Outflow Contribution to AGN Feedback: Observations of QSO SDSS J0838+2955. ApJ706(1):525-534, DOI 10.1088/0004-637X/706/1/525, 0911. 3332

Momose R, Ouchi M, Nakajima K, Ono Y, Shibuya T, Shimasaku K, Yuma S, Mori M, Umemura M (2016) Statistical properties of diffuse Ly $\alpha$ haloes around star-forming galaxies at $z \sim 2$. MNRAS457(3):2318-2330, DOI 10.1093/mnras/stw021, 1509.09001

Morganti R (2012) Using HI Absorption to Trace Outflows from Galaxies and Feeding of AGN. Astrophysics and Space Science Proceedings 25:31, DOI 10.1007/978-3-642-22795-0_4

Morganti R, Oosterloo T (2018) The interstellar and circumnuclear medium of active nuclei traced by $\mathrm{H} \mathrm{i}$ $21 \mathrm{~cm}$ absorption. A\&A Rev.26(1):4, DOI 10.1007/s00159-018-0109-x, 1807.01475

Morganti R, Oosterloo T, Tsvetanov Z (1998) A Radio Study of the Seyfert Galaxy IC 5063: Evidence for Fast Gas Outflow. AJ115(3):915-927, DOI 10.1086/300236, astro-ph/9711285

Morganti R, Tadhunter CN, Oosterloo TA (2005) Fast neutral outflows in powerful radio galaxies: a major source of feedback in massive galaxies. A\&A444(1):L9-L13, DOI 10.1051/0004-6361:200500197, astro-ph/0510263

Morganti R, Holt J, Saripalli L, Oosterloo TA, Tadhunter CN (2007) IC 5063: AGN driven outflow of warm and cold gas. A\&A476(2):735-743, DOI 10.1051/0004-6361:20077888, 0710. 1189

Morganti R, Oosterloo T, Struve C, Saripalli L (2008) A circumnuclear disk of atomic hydrogen in Centaurus A. A\&A485(2):L5-L8, DOI 10.1051/0004-6361:200809974, 0805. 1627

Morganti R, Frieswijk W, Oonk RJB, Oosterloo T, Tadhunter C (2013) Tracing the extreme interplay between radio jets and the ISM in IC 5063. A\&A552:L4, DOI 10.1051/0004-6361/201220734, 1302. 2236

Morganti R, Oosterloo T, Oonk JBR, Frieswijk W, Tadhunter C (2015) The fast molecular outflow in the Seyfert galaxy IC 5063 as seen by ALMA. A\&A580:A1, DOI 10.1051/0004-6361/201525860, 1505.07190

Morganti R, Veilleux S, Oosterloo T, Teng SH, Rupke D (2016) Another piece of the puzzle: The fast H I outflow in Mrk 231. A\&A593:A30, DOI 10.1051/0004-6361/201628978, 1606.01640

Mou G, Yuan F, Bu D, Sun M, Su M (2014) Fermi Bubbles Inflated by Winds Launched from the Hot Accretion Flow in Sgr A*. ApJ790(2):109, DOI 10.1088/0004-637X/790/2/109, 1403.2129

Mould JR, Ridgewell A, Gallagher I John S, Bessell MS, Keller S, Calzetti D, Clarke JT, Trauger JT, Grillmair C, Ballester GE, Burrows CJ, Krist J, Crisp D, Evans R, Griffiths R, Hester JJ, Hoessel JG, Holtzman JA, Scowen PA, Stapelfeldt KR, Sahai R, Watson A, Meadows V (2000) Jet-induced Star Formation in Centaurus A. ApJ536(1):266-276, DOI 10.1086/308927

Mukherjee D, Bicknell GV, Sutherland R, Wagner A (2016) Relativistic jet feedback in high-redshift galaxies - I. Dynamics. MNRAS461(1):967-983, DOI 10.1093/mnras/stw1368, 1606.01143

Mukherjee D, Bicknell GV, Wagner AeY, Sutherland RS, Silk J (2018a) Relativistic jet feedback - III. Feedback on gas discs. MNRAS479(4):5544-5566, DOI 10.1093/mnras/sty1776, 1803.08305

Mukherjee D, Wagner AY, Bicknell GV, Morganti R, Oosterloo T, Nesvadba N, Sutherland RS (2018b) The jet-ISM interactions in IC 5063. MNRAS476(1):80-95, DOI 10.1093/mnras/sty067, 1801.06875

Mulcahy DD, Horneffer A, Beck R, Krause M, Schmidt P, Basu A, Chyzy KT, Dettmar RJ, Haverkorn M, Heald G, Heesen V, Horellou C, Iacobelli M, Nikiel-Wroczyński B, Paladino R, Scaife AMM, Sridhar SS, Strom RG, Tabatabaei FS, Cantwell T, Carey SH, Grainge K, Hickish J, Perrot Y, RazaviGhods N, Scott P, Titterington D (2018) Investigation of the cosmic ray population and magnetic field strength in the halo of NGC 891. A\&A615:A98, DOI 10.1051/0004-6361/201832837, 1804.00752

Muller S, Müller HSP, Black JH, Gérin M, Combes F, Curran S, Falgarone E, Guélin M, Henkel C, Martín S, Menten KM, Roueff E, Aalto S, Beelen A, Wiklind T, Zwaan MA (2017) Detection of $\mathrm{CH}^{+}$, $\mathrm{SH}^{+}$, and their ${ }^{13} \mathrm{C}$ - and ${ }^{34} \mathrm{~S}$-isotopologues toward PKS 1830-211. A\&A606:A109, DOI 10.1051/ 0004-6361/201731405, 1707.07446

Muratov AL, Kereš D, Faucher-Giguère CA, Hopkins PF, Quataert E, Murray N (2015) Gusty, gaseous flows of FIRE: galactic winds in cosmological simulations with explicit stellar feedback. 
MNRAS454(3):2691-2713, DOI 10.1093/mnras/stv2126, 1501.03155

Muratov AL, Kereš D, Faucher-Giguère CA, Hopkins PF, Ma X, Anglés-Alcázar D, Chan TK, Torrey P, Hafen ZH, Quataert E, Murray N (2017) Metal flows of the circumgalactic medium, and the metal budget in galactic haloes. MNRAS468(4):4170-4188, DOI 10.1093/mnras/stx667, 1606.09252

Murray N, Quataert E, Thompson TA (2005) On the Maximum Luminosity of Galaxies and Their Central Black Holes: Feedback from Momentum-driven Winds. ApJ618(2):569-585, DOI 10.1086/426067, astro-ph/0406070

Murray N, Martin CL, Quataert E, Thompson TA (2007) The Ionization State of Sodium in Galactic Winds. ApJ660(1):211-220, DOI 10.1086/512660, astro-ph/0609213

Murray N, Quataert E, Thompson TA (2010) The Disruption of Giant Molecular Clouds by Radiation Pressure \&amp; the Efficiency of Star Formation in Galaxies. ApJ709(1):191-209, DOI 10.1088/ 0004-637X/709/1/191, 0906.5358

Murray N, Ménard B, Thompson TA (2011) Radiation Pressure from Massive Star Clusters as a Launching Mechanism for Super-galactic Winds. ApJ735(1):66, DOI 10.1088/0004-637X/735/1/66, 1005 . 4419

Murthy S, Morganti R, Oosterloo T, Schulz R, Mukherjee D, Wagner AY, Bicknell G, Prandoni I, Shulevski $\mathrm{Aa}$ (2019) Feedback from low-luminosity radio galaxies: B2 0258+35. A\&A629:A58, DOI 10.1051/ 0004-6361/201935931, 1908.00374

Mutch SJ, Croton DJ, Poole GB (2011) The Mid-life Crisis of the Milky Way and M31. ApJ736(2):84, DOI $10.1088 / 0004-637 X / 736 / 2 / 84,1105.2564$

Myers AT, McKee CF, Li PS (2015) The $\mathrm{CH}^{+}$abundance in turbulent, diffuse molecular clouds. MNRAS453(3):2747-2758, DOI 10.1093/mnras/stv1782, 1509.03259

Nakashima S, Koyama K, Wang QD, Enokiya R (2019) X-Ray Observation of a Magnetized Hot Gas Outflow in the Galactic Center Region. ApJ875(1):32, DOI 10.3847/1538-4357/ab0d82, 1903.02571

Narayanan D, Krumholz MR (2017) A physical model for the [C II]-FIR deficit in luminous galaxies. MNRAS467(1):50-67, DOI 10.1093/mnras/stw3218, 1601.05803

Nardini E, Reeves JN, Gofford J, Harrison FA, Risaliti G, Braito V, Costa MT, Matzeu GA, Walton DJ, Behar E, Boggs SE, Christensen FE, Craig WW, Hailey CJ, Matt G, Miller JM, O'Brien PT, Stern D, Turner TJ, Ward MJ (2015) Black hole feedback in the luminous quasar PDS 456. Science 347(6224):860-863, DOI 10.1126/science.1259202, 1502.06636

Nayakshin S, Zubovas K (2012) Quasar feedback: accelerated star formation and chaotic accretion MNRAS427(1):372-378, DOI 10.1111/j.1365-2966.2012.21950.x, 1207.7200

Nedelchev B, Sarzi M, Kaviraj S (2019) The insignificance of Seyfert 2 activity in driving cold-gas galactic winds. MNRAS486(2):1608-1619, DOI 10.1093/mnras/stz934

Neff SG, Eilek JA, Owen FN (2015) The Complex North Transition Region of Centaurus A: A Galactic Wind. ApJ802(2):88, DOI 10.1088/0004-637X/802/2/88, 1502.05236

Nelson D, Pillepich A, Springel V, Pakmor R, Weinberger R, Genel S, Torrey P, Vogelsberger M, Marinacci F, Hernquist L (2019) First Results from the TNG50 Simulation: Galactic outflows driven by supernovae and black hole feedback. MNRASp 2010, DOI 10.1093/mnras/stz2306, 1902.05554

Netzer H (2013) The Physics and Evolution of Active Galactic Nuclei

Neufeld DA (1990) The Transfer of Resonance-Line Radiation in Static Astrophysical Media. ApJ350:216, DOI $10.1086 / 168375$

Nielsen NM, Churchill CW, Kacprzak GG, Murphy MT, Evans JL (2015) MAGIICAT V. Orientation of Outflows and Accretion Determine the Kinematics and Column Densities of the Circumgalactic Medium. ApJ812(1):83, DOI 10.1088/0004-637X/812/1/83, 1505.07167

Nielsen NM, Churchill CW, Kacprzak GG, Murphy MT, Evans JL (2016) MAGIICAT IV. Kinematics of the Circumgalactic Medium and Evidence for Quiescent Evolution Around Red Galaxies. ApJ818(2):171, DOI 10.3847/0004-637X/818/2/171, 1512.06969

Nims J, Quataert E, Faucher-Giguère CA (2015) Observational signatures of galactic winds powered by active galactic nuclei. MNRAS447(4):3612-3622, DOI 10.1093/mnras/stu2648, 1408. 5141

Novikov ID, Thorne KS (1973) Astrophysics of black holes. In: Black Holes (Les Astres Occlus), pp 343-450

Nozawa T, Kozasa T, Habe A (2006) Dust Destruction in the High-Velocity Shocks Driven by Supernovae in the Early Universe. ApJ648(1):435-451, DOI 10.1086/505639, astro-ph/0605193

Nyland K, Alatalo K, Wrobel JM, Young LM, Morganti R, Davis TA, de Zeeuw PT, Deustua S, Bureau M (2013) Detection of a High Brightness Temperature Radio Core in the Active-galactic-nucleusdriven Molecular Outflow Candidate NGC 1266. ApJ779(2):173, DOI 10.1088/0004-637X/779/2/ $173,1310.7588$

Obied G, Zakamska NL, Wylezalek D, Liu G (2016) Giant scattering cones in obscured quasars. 
MNRAS456(3):2861-2876, DOI 10.1093/mnras/stv2850, 1512.03817

Offner SSR, Bisbas TG, Bell TA, Viti S (2014) An alternative accurate tracer of molecular clouds: the 'XCi-factor'. MNRAS440:L81-L85, DOI 10.1093/mnras1/slu013, 1401.5072

Ohyama Y, Sakamoto K, Aalto S, Gallagher I John S (2019) Dusty Superwind from a Galaxy with a Compact Obscured Nucleus: Optical Spectroscopic Study of NGC 4418. ApJ871(2):191, DOI 10. 3847/1538-4357/aaf9a5

Oka T, Geballe TR, Goto M, Usuda T, McCall BJ (2005) Hot and Diffuse Clouds near the Galactic Center Probed by Metastable $\mathrm{H}_{3}{ }^{+}$. ApJ632(2):882-893, DOI 10.1086/432679, astro-ph/0507463

Oka T, Geballe TR, Goto M, Usuda T, Benjamin, McCall J, Indriolo N (2019) The Central 300 pc of the Galaxy Probed by Infrared Spectra of $\mathrm{H}_{3}^{+}$and CO. I. Predominance of Warm and Diffuse Gas and High $\mathrm{H}_{2}$ Ionization Rate. ApJ883(1):54, DOI 10.3847/1538-4357/ab3647, 1910.04762

Olivares V, Salomé P, Combes F, Hamer S, Guillard P, Lehnert MD, Polles F, Beckmann RS, Dubois Y, Donahue M, Edge A, Fabian AC, McNamara B, Rose T, Russell H, Tremblay G, Vantyghem A, Canning REA, Ferland G, Godard B, Hogan M, Peirani S, des Pineau Forets G (2019) Ubiquitous cold and massive filaments in cool core clusters. arXiv e-prints arXiv: 1902.09164, 1902.09164

Onodera M, Carollo CM, Renzini A, Cappellari M, Mancini C, Arimoto N, Daddi E, Gobat R, Strazzullo V, Tacchella S, Yamada Y (2015) The Ages, Metallicities, and Element Abundance Ratios of Massive Quenched Galaxies at $\mathrm{z} \geq 1.6$. ApJ808(2):161, DOI 10.1088/0004-637X/808/2/161, 1411.5023

Oort JH, Spitzer J Lyman (1955) Acceleration of Interstellar Clouds by O-Type Stars. ApJ121:6, DOI $10.1086 / 145958$

Oosterloo T, Raymond Oonk JB, Morganti R, Combes F, Dasyra K, Salomé P, Vlahakis N, Tadhunter C (2017) Properties of the molecular gas in the fast outflow in the Seyfert galaxy IC 5063. A\&A608:A38, DOI 10.1051/0004-6361/201731781, 1710.01570

Oosterloo TA, Morganti R (2005) Anomalous HI kinematics in Centaurus A: Evidence for jet-induced star formation. A\&A429:469-475, DOI 10.1051/0004-6361:20041379, astro-ph/0409500

Oosterloo TA, Morganti R, Tzioumis A, Reynolds J, King E, McCulloch P, Tsvetanov Z (2000) A Strong Jet-Cloud Interaction in the Seyfert Galaxy IC 5063: VLBI Observations. AJ119(5):2085-2091, DOI 10.1086/301358, astro-ph/0002140

Oppenheimer BD, Davies JJ, Crain RA, Wijers NA, Schaye J, Werk JK, Burchett JN, Trayford JW, Horton R (2019) Feedback from supermassive black holes transforms centrals into passive galaxies by ejecting circumgalactic gas. arXiv e-prints arXiv:1904.05904, 1904.05904

Orlando S, Peres G, Reale F, Bocchino F, Rosner R, Plewa T, Siegel A (2005) Crushing of interstellar gas clouds in supernova remnants. I. The role of thermal conduction and radiative losses. A\&A444(2):505-519, DOI 10.1051/0004-6361:20052896, astro-ph/0508638

Orlando S, Bocchino F, Reale F, Peres G, Pagano P (2008) The Importance of Magnetic-Field-Oriented Thermal Conduction in the Interaction of SNR Shocks with Interstellar Clouds. ApJ678(1):274-286, DOI 10.1086/529420, 0801.1403

O’Sullivan D, Martin C, Matuszewski M, Hoadley K, Hamden E, Neill JD, Lin Z, Parihar P (2019) The FLASHES Survey I: Integral Field Spectroscopy of the CGM around $48 z=2.3-3.1$ QSOs. arXiv e-prints arXiv:1911.10740, 1911.10740

Ott J, Weiss A, Henkel C, Walter F (2005) The Temperature Distribution of Dense Molecular Gas in the Center of NGC 253. ApJ629(2):767-780, DOI 10.1086/431661, astro-ph/0505143

Pakmor R, Pfrommer C, Simpson CM, Springel V (2016) Galactic Winds Driven by Isotropic and Anisotropic Cosmic-Ray Diffusion in Disk Galaxies. ApJLett824(2):L30, DOI 10.3847/2041-8205/ 824/2/L30, 1605.00643

Papadopoulos PP, Thi WF, Viti S (2004) CI lines as tracers of molecular gas, and their prospects at high redshifts. MNRAS351(1):147-160, DOI 10.1111/j.1365-2966.2004.07762.x, astro-ph/0403092

Pathak A, Pradhan AC, Sujatha NV, Murthy J (2011) Survey of O VI absorption in the Large Magellanic Cloud. MNRAS412(2):1105-1122, DOI 10.1111/j.1365-2966.2010.17964.x, 1011.0690

Peek JEG, Ménard B, Corrales L (2015) Dust in the Circumgalactic Medium of Low-redshift Galaxies. ApJ813(1):7, DOI 10.1088/0004-637X/813/1/7, 1411.3333

Peeples MS, Corlies L, Tumlinson J, O’Shea BW, Lehner N, O’Meara JM, Howk JC, Earl N, Smith BD, Wise JH, Hummels CB (2019) Figuring Out Gas \&amp; Galaxies in Enzo (FOGGIE). I. Resolving Simulated Circumgalactic Absorption at $2 \leq \mathrm{z} \leq 2.5$. ApJ873(2):129, DOI 10.3847/1538-4357/ ab0654, 1810.06566

Pellegrini EW, Smith JD, Wolfire MG, Draine BT, Crocker AF, Croxall KV, van der Werf P, Dale DA, Rigopoulou D, Wilson CD, Schinnerer E, Groves BA, Kreckel K, Sandstrom KM, Armus L, Calzetti D, Murphy EJ, Walter F, Koda J, Bayet E, Beirao P, Bolatto AD, Bradford M, Brinks E, Hunt 
L, Kennicutt R, Knapen JH, Leroy AK, Rosolowsky E, Vigroux L, Hopwood RHB (2013) Shock Excited Molecules in NGC 1266: ULIRG Conditions at the Center of a Bulge-dominated Galaxy. ApJLett779(2):L19, DOI 10.1088/2041-8205/779/2/L19, 1311. 3993

Peng Y, Maiolino R, Cochrane R (2015) Strangulation as the primary mechanism for shutting down star formation in galaxies. Nature521(7551):192-195, DOI 10.1038/nature14439, 1505.03143

Pereira-Santaella M, Colina L, García-Burillo S, Alonso-Herrero A, Arribas S, Cazzoli S, Emonts B, Piqueras López J, Planesas P, Storchi Bergmann T, Usero A, Villar-Martín M (2016) High-velocity extended molecular outflow in the star-formation dominated luminous infrared galaxy ESO 320-G030. A\&A594:A81, DOI 10.1051/0004-6361/201628875, 1607.03674

Pereira-Santaella M, Colina L, García-Burillo S, Combes F, Emonts B, Aalto S, Alonso-Herrero A, Arribas S, Henkel C, Labiano A, Muller S, Piqueras López J, Rigopoulou D, van der Werf P (2018) Spatially resolved cold molecular outflows in ULIRGs. A\&A616:A171, DOI 10.1051/0004-6361/201833089, 1805.03667

Perna M, Lanzuisi G, Brusa M, Cresci G, Mignoli M (2017) An X-ray/SDSS sample. II. AGN-driven outflowing gas plasma properties. A\&A606:A96, DOI 10.1051/0004-6361/201730819, 1705.08388

Péroux C, Meiring JD, Kulkarni VP, Ferlet R, Khare P, Lauroesch JT, Vladilo G, York DG (2006) Metalrich damped/subdamped Lyman $\alpha$ quasar absorbers at z \&lt; 1. MNRAS372(1):369-380, DOI 10. 1111/j.1365-2966.2006.10865.x, astro-ph/0607561

Perrotta S, D’Odorico V, Prochaska JX, Cristiani S, Cupani G, Ellison S, López S, Becker GD, Berg TAM, Christensen L, Denney KD, Hamann F, Pâris I, Vestergaard M, Worseck G (2016) Nature and statistical properties of quasar associated absorption systems in the XQ-100 Legacy Survey. MNRAS462(3):3285-3301, DOI 10.1093/mnras/stw1703, 1605.04607

Perrotta S, Hamann F, Zakamska NL, Alexand roff RM, Rupke D, Wylezalek D (2019) ERQs are the BOSS of quasar samples: the highest velocity [O III] quasar outflows. MNRAS488(3):4126-4148, DOI 10.1093/mnras/stz1993, 1906.00980

Pettini M, Rix SA, Steidel CC, Adelberger KL, Hunt MP, Shapley AE (2002) New Observations of the Interstellar Medium in the Lyman Break Galaxy MS 1512-cB58. ApJ569(2):742-757, DOI 10.1086/ 339355, astro-ph/0110637

Pfrommer C, Pakmor R, Schaal K, Simpson CM, Springel V (2017) Simulating cosmic ray physics on a moving mesh. MNRAS465(4):4500-4529, DOI 10.1093/mnras/stw2941, 1604.07399

Phillips AC (1993) Nuclear and large-Scale Outflows in NGC 1808. AJ105:486, DOI 10.1086/116447

Planck Collaboration, Ade PAR, Aghanim N, Arnaud M, Ashdown M, Atrio-Barandela F, Aumont J, Baccigalupi C, Balbi A, Banday AJ, Barreiro RB, Bartlett JG, Battaner E, Benabed K, Benoît A, Bernard JP, Bersanelli M, Bonaldi A, Bond JR, Borrill J, Bouchet FR, Burigana C, Cabella P, Cardoso JF, Catalano A, Cayón L, Chary RR, Chiang LY, Christensen PR, Clements DL, Colombo LPL, Coulais A, Crill BP, Cuttaia F, Danese L, D'Arcangelo O, Davis RJ, de Bernardis P, de Gasperis G, de Rosa A, de Zotti G, Delabrouille J, Dickinson C, Diego JM, Dobler G, Dole H, Donzelli S, Doré O, Dörl U, Douspis M, Dupac X, Efstathiou G, Enßlin TA, Eriksen HK, Finelli F, Forni O, Frailis M, Franceschi E, Galeotta S, Ganga K, Giard M, Giardino G, González-Nuevo J, Górski KM, Gratton S, Gregorio A, Gruppuso A, Hansen FK, Harrison D, Helou G, Henrot-Versillé S, Hernández-Monteagudo C, Hildebrandt SR, Hivon E, Hobson M, Holmes WA, Hornstrup A, Hovest W, Huffenberger KM, Jaffe TR, Jagemann T, Jewell J, Jones WC, Juvela M, Keihänen E, Knoche J, Knox L, Kunz M, Kurki-Suonio H, Lagache G, Lähteenmäki A, Lamarre JM, Lasenby A, Lawrence CR, Leach S, Leonardi R, Lilje PB, Linden-Vørnle M, López-Caniego M, Lubin PM, Macías-Pérez JF, Maffei B, Maino D, Mand olesi N, Maris M, Marshall DJ, Martin PG, Martínez-González E, Masi S, Massardi M, Matarrese S, Matthai F, Mazzotta P, Meinhold PR, Melchiorri A, Mendes L, Mennella A, Mitra S, Moneti A, Montier L, Morgante G, Munshi D, Murphy JA, Naselsky P, Natoli P, Nørgaard-Nielsen HU, Noviello F, Novikov D, Novikov I, Osborne S, Pajot F, Paladini R, Paoletti D, Partridge B, Pearson TJ, Perdereau O, Perrotta F, Piacentini F, Piat M, Pierpaoli E, Pietrobon D, Plaszczynski S, Pointecouteau E, Polenta G, Ponthieu N, Popa L, Poutanen T, Pratt GW, Prunet S, Puget JL, Rachen JP, Rebolo R, Reinecke M, Renault C, Ricciardi S, Riller T, Ristorcelli I, Rocha G, Rosset C, Rubiño-Martín JA, Rusholme B, Sandri M, Savini G, Schaefer BM, Scott D, Smoot GF, Spencer L, Stivoli F, Sudiwala R, SuurUski AS, Sygnet JF, Tauber JA, Terenzi L, Toffolatti L, Tomasi M, Tristram M, Türler M, Umana G, Valenziano L, Van Tent B, Vielva P, Villa F, Vittorio N, Wade LA, Wandelt BD, White M, Yvon D, Zacchei A, Zonca A (2013) Planck intermediate results. IX. Detection of the Galactic haze with Planck. A\&A554:A139, DOI 10.1051/0004-6361/201220271, 1208. 5483

Planck Collaboration, Abergel A, Ade PAR, Aghanim N, Alves MIR, Aniano G, Armitage-Caplan C, Arnaud M, Ashdown M, Atrio-Barand ela F, Aumont J, Baccigalupi C, Banday AJ, Barreiro RB, 
Bartlett JG, Battaner E, Benabed K, Benoît A, Benoit-Lévy A, Bernard JP, Bersanelli M, Bielewicz P, Bobin J, Bock JJ, Bonaldi A, Bond JR, Borrill J, Bouchet FR, Boulanger F, Bridges M, Bucher M, Burigana C, Butler RC, Cardoso JF, Catalano A, Chamballu A, Chary RR, Chiang HC, Chiang LY, Christensen PR, Church S, Clemens M, Clements DL, Colombi S, Colombo LPL, Combet C, Coucho F, Coulais A, Crill BP, Curto A, Cuttaia F, Danese L, Davies RD, Davis RJ, de Bernardis P, de Rosa A, de Zotti G, Delabrouille J, Delouis JM, Désert FX, Dickinson C, Diego JM, Dole H, Donzelli S, Doré O, Douspis M, Draine BT, Dupac X, Efstathiou G, Enßlin TA, Eriksen HK, Falgarone E, Finelli F, Forni O, Frailis M, Fraisse AA, Franceschi E, Galeotta S, Ganga K, Ghosh T, Giard M, Giardino G, Giraud-Héraud Y, González-Nuevo J, Górski KM, Gratton S, Gregorio A, Grenier IA, Gruppuso A, Guillet V, Hansen FK, Hanson D, Harrison DL, Helou G, Henrot-Versillé S, HernándezMonteagudo C, Herranz D, Hildebrand t SR, Hivon E, Hobson M, Holmes WA, Hornstrup A, Hovest W, Huffenberger KM, Jaffe AH, Jaffe TR, Jewell J, Joncas G, Jones WC, Juvela M, Keihänen E, Keskitalo R, Kisner TS, Knoche J, Knox L, Kunz M, Kurki-Suonio H, Lagache G, Lähteenmäki A, Lamarre JM, Lasenby A, Laureijs RJ, Lawrence CR, Leonardi R, León-Tavares J, Lesgourgues J, Levrier F, Liguori M, Lilje PB, Linden-Vørnle M, López-Caniego M, Lubin PM, Macías-Pérez JF, Maffei B, Maino D, Mand olesi N, Maris M, Marshall DJ, Martin PG, Martínez-González E, Masi S, Massardi M, Matarrese S, Matthai F, Mazzotta P, McGehee P, Melchiorri A, Mendes L, Mennella A, Migliaccio M, Mitra S, Miville-Deschênes MA, Moneti A, Montier L, Morgante G, Mortlock D, Munshi D, Murphy JA, Naselsky P, Nati F, Natoli P, Netterfield CB, Nørgaard-Nielsen HU, Noviello F, Novikov D, Novikov I, Osborne S, Oxborrow CA, Paci F, Pagano L, Pajot F, Paladini R, Paoletti D, Pasian F, Patanchon G, Perdereau O, Perotto L, Perrotta F, Piacentini F, Piat M, Pierpaoli E, Pietrobon D, Plaszczynski S, Pointecouteau E, Polenta G, Ponthieu N, Popa L, Poutanen T, Pratt GW, Prézeau G, Prunet S, Puget JL, Rachen JP, Reach WT, Rebolo R, Reinecke M, Remazeilles M, Renault C, Ricciardi S, Riller T, Ristorcelli I, Rocha G, Rosset C, Roudier G, Rowan-Robinson M, Rubiño-Martín JA, Rusholme B, Sandri M, Santos D, Savini G, Scott D, Seiffert MD, Shellard EPS, Spencer LD, Starck JL, Stolyarov V, Stompor R, Sudiwala R, Sunyaev R, Sureau F, Sutton D, Suur-Uski AS, Sygnet JF, Tauber JA, Tavagnacco D, Terenzi L, Toffolatti L, Tomasi M, Tristram M, Tucci M, Tuovinen J, Türler M, Umana G, Valenziano L, Valiviita J, Van Tent B, Verstraete L, Vielva P, Villa F, Vittorio N, Wade LA, Wandelt BD, Welikala N, Ysard N, Yvon D, Zacchei A, Zonca A (2014) Planck 2013 results. XI. All-sky model of thermal dust emission. A\&A571:A11, DOI 10.1051/0004-6361/201323195, 1312.1300

Pogge RW (1988) Extended Ionized Gas in the Seyfert 2 Galaxy NGC 4388. ApJ332:702, DOI 10.1086/ 166687

Pointon SK, Kacprzak GG, Nielsen NM, Muzahid S, Murphy MT, Churchill CW, Charlton JC (2019) Relationship between the Metallicity of the Circumgalactic Medium and Galaxy Orientation. arXiv e-prints arXiv:1907.05557, 1907.05557

Ponti G, Terrier R, Goldwurm A, Belanger G, Trap G (2010) Discovery of a Superluminal Fe K Echo at the Galactic Center: The Glorious Past of Sgr A* Preserved by Molecular Clouds. ApJ714(1):732-747, DOI 10.1088/0004-637X/714/1/732, 1003.2001

Ponti G, Hofmann F, Churazov E, Morris MR, Haberl F, Nandra K, Terrier R, Clavel M, Goldwurm A (2019) An X-ray chimney extending hundreds of parsecs above and below the Galactic Centre. Nature567(7748):347-350, DOI 10.1038/s41586-019-1009-6, 1904.05969

Prochaska JX, Kasen D, Rubin K (2011) Simple Models of Metal-line Absorption and Emission from Cool Gas Outflows. ApJ734(1):24, DOI 10.1088/0004-637X/734/1/24, 1102 . 3444

Prochaska JX, Lau MW, Hennawi JF (2014) Quasars Probing Quasars. VII. The Pinnacle of the Cool Circumgalactic Medium Surrounds Massive $z \sim 2$ Galaxies. ApJ796(2):140, DOI 10.1088/0004-637X/ 796/2/140, 1409.6344

Puxley PJ, Hawarden TG, Mountain CM (1990) Molecular and Atomic Hydrogen Line Emission from Star-forming Galaxies. ApJ364:77, DOI 10.1086/169386

Qin SL, Schilke P, Wu J, Wu Y, Liu T, Liu Y, Sánchez-Monge Á (2015) SMA Observations of the W3(OH) Complex: Physical and Chemical Differentiation Between W3( $\left.\mathrm{H}_{2} \mathrm{O}\right)$ and W3(OH). ApJ803(1):39, DOI 10.1088/0004-637X/803/1/39, 1502.04467

Qu Z, Bregman JN, Hodges-Kluck EJ (2019) HST/COS Observations of the Warm Ionized Gaseous Halo of NGC 891. ApJ876(2):101, DOI 10.3847/1538-4357/ab17df, 1904.04716

Quider AM, Pettini M, Shapley AE, Steidel CC (2009) The ultraviolet spectrum of the gravitationally lensed galaxy 'the Cosmic Horseshoe': a close-up of a star-forming galaxy at $z \sim 2$. MNRAS398(3):1263-1278, DOI 10.1111/j.1365-2966.2009.15234.x, 0906. 2412

Quillen AC, Bland-Hawthorn J, Brookes MH, Werner MW, Smith JD, Stern D, Keene J, Lawrence CR 
(2006) Discovery of a 500 Parsec Shell in the Nucleus of Centaurus A. ApJLett641(1):L29-L32, DOI $10.1086 / 503670$, astro-ph/0601147

Quiret S, Péroux C, Zafar T, Kulkarni VP, Jenkins EB, Milliard B, Rahmani H, Popping A, Rao SM, Turnshek DA, Monier EM (2016) The ESO UVES advanced data products quasar sample - VI. Subdamped Lyman $\alpha$ metallicity measurements and the circumgalactic medium. MNRAS458(4):40744121, DOI 10.1093/mnras/stw524, 1602.02564

Rahmani H, Péroux C, Turnshek DA, Rao SM, Quiret S, Hamilton TS, Kulkarni VP, Monier EM, Zafar T (2016) A study of the circumgalactic medium at $z \sim 0.6$ using damped Lyman $\alpha$ galaxies. MNRAS463(1):980-1007, DOI 10.1093/mnras/stw1965, 1609.03843

Redman MP, Al-Mostafa ZA, Meaburn J, Bryce M (2003) High-speed outflows driven by the 30 Doradus starburst. MNRAS344(3):741-747, DOI 10.1046/j.1365-8711.2003.06865.x, astro-ph/0308213

Rees MJ (1988) Tidal disruption of stars by black holes of $10^{6}-10^{8}$ solar masses in nearby galaxies. Nature333(6173):523-528, DOI 10.1038/333523a0

Rekola R, Richer MG, McCall ML, Valtonen MJ, Kotilainen JK, Flynn C (2005) Distance to NGC 253 based on the planetary nebula luminosity function. MNRAS361(1):330-336, DOI 10.1111/j. 1365-2966.2005.09166.x

Reynolds C, Punsly B, Kharb P, O’Dea CP, Wrobel J (2009) VLBA Observations of Sub-Parsec Structure in Mrk 231: Interaction Between a Relativistic Jet and a BAL Wind. ApJ706(1):851-865, DOI 10. 1088/0004-637X/706/1/851, 0909. 5096

Reynolds C, Punsly B, O'Dea CP, Hurley-Walker N (2013) A Blazar-like Radio Flare in Mrk 231. ApJLett776(2):L21, DOI 10.1088/2041-8205/776/2/L21, 1307.8314

Richings AJ, Faucher-Giguère CA (2018a) Radiative cooling of swept-up gas in AGN-driven galactic winds and its implications for molecular outflows. MNRAS478(3):3100-3119, DOI 10.1093/mnras/ sty $1285,1710.09433$

Richings AJ, Faucher-Giguère CA (2018b) The origin of fast molecular outflows in quasars: molecule formation in AGN-driven galactic winds. MNRAS474(3):3673-3699, DOI 10.1093/mnras/stx3014, 1706.03784

Rickards Vaught RJ, Rubin KHR, Arrigoni Battaia F, Prochaska JX, Hennawi JF (2019) A VLT/FORS2 Narrowband Imaging Search for Mg II Emission around $z \sim 0.7$ Galaxies. ApJ879(1):7, DOI 10. 3847/1538-4357/ab211f, 1905.05746

Rigby JR, Bayliss MB, Chisholm J, Bordoloi R, Sharon K, Gladders MD, Johnson T, Paterno-Mahler R, Wuyts E, Dahle H, Acharyya A (2018a) The Magellan Evolution of Galaxies Spectroscopic and Ultraviolet Reference Atlas (MegaSaura). II. Stacked Spectra. ApJ853(1):87, DOI 10.3847/1538-4357/ aaa2fc, 1710.07499

Rigby JR, Bayliss MB, Sharon K, Gladders MD, Chisholm J, Dahle H, Johnson T, Paterno-Mahler R, Wuyts E, Kelson DD (2018b) The Magellan Evolution of Galaxies Spectroscopic and Ultraviolet Reference Atlas (MegaSaura). I. The Sample and the Spectra. AJ155(3):104, DOI 10.3847/1538-3881/ aaa2ff, 1710.07294

Roberts-Borsani G, Saintonge A, Masters KL, Stark DV (2020) Outflows in Star-forming Galaxies: Stacking Analyses of Resolved Winds and the Relation to Their Hosts' Properties. arXiv e-prints arXiv:2002.05724, 2002.05724

Roberts-Borsani GW, Saintonge A (2019) The prevalence and properties of cold gas inflows and outflows around galaxies in the local Universe. MNRAS482(3):4111-4145, DOI 10.1093/mnras/sty2824, 1807.07575

Rodríguez del Pino B, Arribas S, Piqueras López J, Villar-Martín M, Colina L (2019) Properties of ionized outflows in MaNGA DR2 galaxies. MNRAS486(1):344-359, DOI 10.1093/mnras/stz816, 1903.07432

Rodríguez-Fernández NJ, Tafalla M, Gueth F, Bachiller R (2010) HNCO enhancement by shocks in the L1157 molecular outflow. A\&A516:A98, DOI 10.1051/0004-6361/201013997, 1003.4219

Roman-Duval J, Gordon KD, Meixner M, Bot C, Bolatto A, Hughes A, Wong T, Babler B, Bernard JP, Clayton GC, Fukui Y, Galametz M, Galliano F, Glover S, Hony S, Israel F, Jameson K, Lebouteiller V, Lee MY, Li A, Madden S, Misselt K, Montiel E, Okumura K, Onishi T, Panuzzo P, Reach W, Remy-Ruyer A, Robitaille T, Rubio M, Sauvage M, Seale J, Sewilo M, Staveley-Smith L, Zhukovska S (2014) Dust and Gas in the Magellanic Clouds from the HERITAGE Herschel Key Project. II. Gasto-dust Ratio Variations across Interstellar Medium Phases. ApJ797(2):86, DOI 10.1088/0004-637X/ $797 / 2 / 86,1411.4552$

Roos O, Juneau S, Bournaud F, Gabor JM (2015) Thermal and Radiative Active Galactic Nucleus Feedback have a Limited Impact on Star Formation in High-redshift Galaxies. ApJ800(1):19, DOI 
$10.1088 / 0004-637 \mathrm{X} / 800 / 1 / 19,1405.7971$

Rosdahl J, Teyssier R (2015) A scheme for radiation pressure and photon diffusion with the M1 closure in RAMSES-RT. MNRAS449(4):4380-4403, DOI 10.1093/mnras/stv567, 1411.6440

Rosenberg MJF, van der Werf PP, Aalto S, Armus L, Charmandaris V, Díaz-Santos T, Evans AS, Fischer J, Gao Y, González-Alfonso E, Greve TR, Harris AI, Henkel C, Israel FP, Isaak KG, Kramer C, Meijerink R, Naylor DA, Sanders DB, Smith HA, Spaans M, Spinoglio L, Stacey GJ, Veenendaal I, Veilleux S, Walter F, Weiß A, Wiedner MC, van der Wiel MHD, Xilouris EM (2015) The Herschel Comprehensive (U)LIRG Emission Survey (HERCULES): CO Ladders, Fine Structure Lines, and Neutral Gas Cooling. ApJ801(2):72, DOI 10.1088/0004-637X/801/2/72, 1501.02985

Roussel H, Helou G, Smith JD, Draine BT, Hollenbach DJ, Moustakas J, Spoon HW, Kennicutt RC, Rieke GH, Walter F, Armus L, Dale DA, Sheth K, Bendo GJ, Engelbracht CW, Gordon KD, Meyer MJ, Regan MW, Murphy EJ (2006) The Opaque Nascent Starburst in NGC 1377: Spitzer SINGS Observations. ApJ646(2):841-857, DOI 10.1086/505038, astro-ph/0604267

Rubin KHR, Weiner BJ, Koo DC, Martin CL, Prochaska JX, Coil AL, Newman JA (2010) The Persistence of Cool Galactic Winds in High Stellar Mass Galaxies between $z \sim 1.4$ and $\sim 1$. ApJ719(2):15031525, DOI 10.1088/0004-637X/719/2/1503, 0912.2343

Rubin KHR, Prochaska JX, Ménard B, Murray N, Kasen D, Koo DC, Phillips AC (2011) Low-ionization Line Emission from a Starburst Galaxy: A New Probe of a Galactic-scale Outflow. ApJ728(1):55, DOI 10.1088/0004-637X/728/1/55, 1008. 3397

Rubin KHR, Prochaska JX, Koo DC, Phillips AC, Martin CL, Winstrom LO (2014) Evidence for Ubiquitous Collimated Galactic-scale Outflows along the Star-forming Sequence at $z \sim 0.5$. ApJ794(2):156, DOI 10.1088/0004-637X/794/2/156, 1307.1476

Rubin KHR, Diamond-Stanic AM, Coil AL, Crighton NHM, Moustakas J (2018a) Galaxies Probing Galaxies in PRIMUS. I. Sample, Spectroscopy, and Characteristics of the $z \sim 0.5 \mathrm{Mg}$ II-absorbing Circumgalactic Medium. ApJ853(1):95, DOI 10.3847/1538-4357/aa9792, 1711.08807

Rubin KHR, Diamond-Stanic AM, Coil AL, Crighton NHM, Stewart KR (2018b) Galaxies Probing Galaxies in PRIMUS. II. The Coherence Scale of the Cool Circumgalactic Medium. ApJ868(2):142, DOI 10.3847/1538-4357/aad566, 1806.08801

Rudie GC, Steidel CC, Trainor RF, Rakic O, Bogosavljević M, Pettini M, Reddy N, Shapley AE, Erb DK, Law DR (2012) The Gaseous Environment of High-z Galaxies: Precision Measurements of Neutral Hydrogen in the Circumgalactic Medium of $z \sim 2-3$ Galaxies in the Keck Baryonic Structure Survey. ApJ750(1):67, DOI 10.1088/0004-637X/750/1/67, 1202.6055

Rudie GC, Steidel CC, Pettini M, Trainor RF, Strom AL, Hummels CB, Reddy NA, Shapley AE (2019) The Column Density, Kinematics, and Thermal State of Metal-Bearing Gas within the Virial Radius of $z \sim 2$ Star-Forming Galaxies in the Keck Baryonic Structure Survey. arXiv e-prints arXiv:1903.00004, 1903.00004

Ruiz A, Rodriguez LF, Canto J, Mirabel IF (1992) High-Velocity OH in Absorption: A New Tracer of Shocked Gas in Outflows? ApJ398:139, DOI 10.1086/171843

Rupke D (2018) A Review of Recent Observations of Galactic Winds Driven by Star Formation. Galaxies 6(4):138, DOI 10.3390/galaxies6040138, 1812.05184

Rupke DS, Veilleux S, Sanders DB (2002) Keck Absorption-Line Spectroscopy of Galactic Winds in Ultraluminous Infrared Galaxies. ApJ570(2):588-609, DOI 10.1086/339789, astro-ph/0201371

Rupke DS, Veilleux S, Sanders DB (2005a) Outflows in Active Galactic Nucleus/Starburst-Composite Ultraluminous Infrared Galaxies1,. ApJ632(2):751-780, DOI 10.1086/444451, astro-ph/0507037

Rupke DS, Veilleux S, Sanders DB (2005b) Outflows in Infrared-Luminous Starbursts at z \&lt; 0.5. I. Sample, Na I D Spectra, and Profile Fitting. ApJSupp.160(1):87-114, DOI 10.1086/432886, astro-ph/0506610

Rupke DS, Veilleux S, Sanders DB (2005c) Outflows in Infrared-Luminous Starbursts at z \&lt; 0.5. II. Analysis and Discussion. ApJSupp.160(1):115-148, DOI 10.1086/432889, astro-ph/0506611

Rupke DSN, Veilleux S (2011) Integral Field Spectroscopy of Massive, Kiloparsec-scale Outflows in the Infrared-luminous QSO Mrk 231. ApJLett729(2):L27, DOI 10.1088/2041-8205/729/2/L27, 1102. 4349

Rupke DSN, Veilleux S (2013a) Breaking the Obscuring Screen: A Resolved Molecular Outflow in a Buried QSO. ApJLett775(1):L15, DOI 10.1088/2041-8205/775/1/L15, 1308.4988

Rupke DSN, Veilleux S (2013b) The Multiphase Structure and Power Sources of Galactic Winds in Major Mergers. ApJ768(1):75, DOI 10.1088/0004-637X/768/1/75, 1303.6866

Rupke DSN, Veilleux S (2015) Spatially Extended Na I D Resonant Emission and Absorption in the Galactic Wind of the Nearby Infrared-Luminous Quasar F05189-2524. ApJ801(2):126, DOI 10.1088/ 
0004-637X/801/2/126, 1411.3744

Rupke DSN, Gültekin K, Veilleux S (2017) Quasar-mode Feedback in Nearby Type 1 Quasars: Ubiquitous Kiloparsec-scale Outflows and Correlations with Black Hole Properties. ApJ850(1):40, DOI 10.3847/ $1538-4357 / \mathrm{aa} 94 \mathrm{~d} 1,1708.05139$

Rupke DSN, Coil A, Geach JE, Tremonti C, Diamond-Stanic AM, George ER, Hickox RC, Kepley AA, Leung G, Moustakas J, Rudnick G, Sell PH (2019) A 100-kiloparsec wind feeding the circumgalactic medium of a massive compact galaxy. Nature574(7780):643-646, DOI 10.1038/s41586-019-1686-1

Russell HR, McNamara BR, Edge AC, Nulsen PEJ, Main RA, Vantyghem AN, Combes F, Fabian AC, Murray N, Salomé P, Wilman RJ, Baum SA, Donahue M, O’Dea CP, Oonk JBR, Tremblay GR, Voit GM (2014) Massive Molecular Gas Flows in the A1664 Brightest Cluster Galaxy. ApJ784(1):78, DOI 10.1088/0004-637X/784/1/78, 1309.0014

Russell HR, McDonald M, McNamara BR, Fabian AC, Nulsen PEJ, Bayliss MB, Benson BA, Brodwin M, Carlstrom JE, Edge AC, Hlavacek-Larrondo J, Marrone DP, Reichardt CL, Vieira JD (2017a) Alma Observations of Massive Molecular Gas Filaments Encasing Radio Bubbles in the Phoenix Cluster. ApJ836(1):130, DOI 10.3847/1538-4357/836/1/130, 1611.00017

Russell HR, McNamara BR, Fabian AC, Nulsen PEJ, Combes F, Edge AC, Hogan MT, McDonald M, Salomé P, Tremblay G, Vantyghem AN (2017b) Close entrainment of massive molecular gas flows by radio bubbles in the central galaxy of Abell 1795. MNRAS472(4):4024-4037, DOI 10.1093/mnras/ stx2255, 1708.08935

Russell HR, McNamara BR, Fabian AC, Nulsen PEJ, Combes F, Edge AC, Madar M, Olivares V, Salome P, Vantyghem AN (2019) Driving massive molecular gas flows in central cluster galaxies with AGN feedback. arXiv e-prints arXiv:1902.09227, 1902.09227

Ruszkowski M, Yang HYK, Zweibel E (2014) Fermi bubble simulations: black hole feedback in the Milky Way. In: Sjouwerman LO, Lang CC, Ott J (eds) The Galactic Center: Feeding and Feedback in a Normal Galactic Nucleus, IAU Symposium, vol 303, pp 390-394, DOI 10.1017/S1743921314000994, 1311.6159

Ruszkowski M, Yang HYK, Zweibel E (2017) Global Simulations of Galactic Winds Including Cosmicray Streaming. ApJ834(2):208, DOI 10.3847/1538-4357/834/2/208, 1602.04856

Sadowski A, Narayan R (2015) Powerful radiative jets in supercritical accretion discs around non-spinning black holes. MNRAS453(3):3213-3221, DOI 10.1093/mnras/stv1802, 1503.00654

Sadowski A, Narayan R (2016) Three-dimensional simulations of supercritical black hole accretion discs - luminosities, photon trapping and variability. MNRAS456(4):3929-3947, DOI 10.1093/mnras/ stv2941, 1509.03168

Sadowski A, Narayan R, Penna R, Zhu Y (2013) Energy, momentum and mass outflows and feedback from thick accretion discs around rotating black holes. MNRAS436(4):3856-3874, DOI 10.1093/ mnras/stt1881, 1307.1143

Sadowski A, Narayan R, McKinney JC, Tchekhovskoy A (2014) Numerical simulations of supercritical black hole accretion flows in general relativity. MNRAS439(1):503-520, DOI 10.1093/mnras/ stt2479, 1311.5900

Sadowski A, Lasota JP, Abramowicz MA, Narayan R (2016) Energy flows in thick accretion discs and their consequences for black hole feedback. MNRAS456(4):3915-3928, DOI 10.1093/mnras/stv2854, 1510.08845

Saintonge A, Kauffmann G, Wang J, Kramer C, Tacconi LJ, Buchbender C, Catinella B, Graciá-Carpio J, Cortese L, Fabello S, Fu J, Genzel R, Giovanelli R, Guo Q, Haynes MP, Heckman TM, Krumholz MR, Lemonias J, Li C, Moran S, Rodriguez-Fernand ez N, Schiminovich D, Schuster K, Sievers A (2011) COLD GASS, an IRAM legacy survey of molecular gas in massive galaxies - II. The non-universality of the molecular gas depletion time-scale. MNRAS415(1):61-76, DOI 10.1111/j.1365-2966.2011. 18823.x, 1104.0019

Saito T, Iono D, Espada D, Nakanishi K, Ueda J, Sugai H, Takano S, Yun MS, Imanishi M, Ohashi S, Lee M, Hagiwara Y, Motohara K, Kawabe R (2017) Merger-induced Shocks in the Nearby LIRG VV 114 through Methanol Observations with ALMA. ApJ834(1):6, DOI 10.3847/1538-4357/834/1/6, 1611.01156

Sakamoto K, Ho PTP, Peck AB (2006) Imaging Molecular Gas in the Luminous Merger NGC 3256: Detection of High-Velocity Gas and Twin Gas Peaks in the Double Nucleus. ApJ644(2):862-878, DOI 10.1086/503827, astro-ph/0603079

Sakamoto K, Wang J, Wiedner MC, Wang Z, Peck AB, Zhang Q, Petitpas GR, Ho PTP, Wilner DJ (2008) Submillimeter Array Imaging of the $\mathrm{CO}(3-2)$ Line and $860 \mu \mathrm{m}$ Continuum of Arp 220: Tracing the Spatial Distribution of Luminosity. ApJ684(2):957-977, DOI 10.1086/590484, 0806.0217 
Sakamoto K, Aalto S, Evans AS, Wiedner MC, Wilner DJ (2010) Vibrationally Excited HCN in the Luminous Infrared Galaxy NGC 4418. ApJLett725(2):L228-L233, DOI 10.1088/2041-8205/725/2/L228, 1011.6449

Sakamoto K, Aalto S, Costagliola F, Martín S, Ohyama Y, Wiedner MC, Wilner DJ (2013) Submillimeter Interferometry of the Luminous Infrared Galaxy NGC 4418: A Hidden Hot Nucleus with an Inflow and an Outflow. ApJ764(1):42, DOI 10.1088/0004-637X/764/1/42, 1301. 1878

Sakamoto K, Aalto S, Combes F, Evans A, Peck A (2014) An Infrared-luminous Merger with Two Bipolar Molecular Outflows: ALMA and SMA Observations of NGC 3256. ApJ797(2):90, DOI 10.1088/ 0004-637X/797/2/90, 1403.7117

Sakamoto K, Aalto S, Barcos-Muñoz L, Costagliola F, Evans AS, Harada N, Martín S, Wiedner M, Wilner D (2017) Resolved Structure of the Arp 220 Nuclei at $\lambda \approx 3 \mathrm{~mm}$. ApJ849(1):14, DOI 10.3847/1538-4357/aa8f4b, 1709.08537

Salomé Q, Salomé P, Combes F, Hamer S, Heywood I (2016) Star formation efficiency along the radio jet in Centaurus A. A\&A586:A45, DOI 10.1051/0004-6361/201526409, 1511.04310

Salomé Q, Salomé P, Gusdorf A, Combes F (2019) Physical conditions in Centaurus A's northern filaments. I. APEX mid-J CO observations of CO-bright regions. A\&A627:A6, DOI 10.1051/0004-6361/ 201833866, 1901.11148

Salter CJ, Ghosh T, Catinella B, Lebron M, Lerner MS, Minchin R, Momjian E (2008) The Arecibo ARP 220 Spectral Census. I. Discovery of the Pre-Biotic Molecule Methanimine and New Cm-Wavelength Transitions of Other Molecules. AJ136(1):389-399, DOI 10.1088/0004-6256/136/1/389, 0805.1205

Santoro F, Oonk JBR, Morganti R, Oosterloo T (2015a) The jet-ISM interaction in the outer filament of Centaurus A. A\&A574:A89, DOI 10.1051/0004-6361/201425103, 1411.4639

Santoro F, Oonk JBR, Morganti R, Oosterloo TA, Tremblay G (2015b) The outer filament of Centaurus A as seen by MUSE. A\&A575:L4, DOI 10.1051/0004-6361/201425511, 1501.06906

Santoro F, Oonk JBR, Morganti R, Oosterloo TA, Tadhunter C (2016) Embedded star formation in the extended narrow line region of Centaurus A: Extreme mixing observed by MUSE. A\&A590:A37, DOI 10.1051/0004-6361/201628353, 1604.03891

Sarangi A, Dwek E, Kazanas D (2019) Dust Formation in AGN Winds. ApJ885(2):126, DOI 10.3847/ 1538-4357/ab46a9

Sarzi M, Kaviraj S, Nedelchev B, Tiffany J, Shabala SS, Deller AT, Middelberg E (2016) Cold-gas outflows in typical low-redshift galaxies are driven by star formation, not AGN. MNRAS456(1):L25-L29, DOI 10.1093/mnrasl/slv165, 1510.06738

Savage BD, Bohlin RC (1979) The depletion of interstellar gaseous iron. ApJ229:136-146, DOI 10.1086/ 156938

Savage BD, Sembach KR (1996) Interstellar Abundances from Absorption-Line Observations with the Hubble Space Telescope. ARA\&A34:279-330, DOI 10.1146/annurev.astro.34.1.279

Savage BD, Kim TS, Fox AJ, Massa D, Bordoloi R, Jenkins EB, Lehner N, Bland-Hawthorn J, Lockman FJ, Hernandez S, Wakker BP (2017) Probing the Outflowing Multiphase Gas $~ 1 \mathrm{kpc}$ below the Galactic Center. ApJSupp.232(2):25, DOI 10.3847/1538-4365/aa8f4c, 1707.06942

Scannapieco E (2017) The Production of Cold Gas Within Galaxy Outflows. ApJ837(1):28, DOI 10.3847/ $1538-4357 /$ aa5d0d, 1702.00793

Scannapieco E, Brüggen M (2015) The Launching of Cold Clouds by Galaxy Outflows. I. Hydrodynamic Interactions with Radiative Cooling. ApJ805(2):158, DOI 10.1088/0004-637X/805/2/158, 1503.06800

Schawinski K, Urry CM, Simmons BD, Fortson L, Kaviraj S, Keel WC, Lintott CJ, Masters KL, Nichol RC, Sarzi M, Skibba R, Treister E, Willett KW, Wong OI, Yi SK (2014) The green valley is a red herring: Galaxy Zoo reveals two evolutionary pathways towards quenching of star formation in earlyand late-type galaxies. MNRAS440(1):889-907, DOI 10.1093/mnras/stu327, 1402.4814

Schawinski K, Koss M, Berney S, Sartori LF (2015) Active galactic nuclei flicker: an observational estimate of the duration of black hole growth phases of $\sim 10^{5} \mathrm{yr}$. MNRAS451(3):2517-2523, DOI 10.1093/mnras/stv1136, 1505.06733

Schaye J, Crain RA, Bower RG, Furlong M, Schaller M, Theuns T, Dalla Vecchia C, Frenk CS, McCarthy IG, Helly JC, Jenkins A, Rosas-Guevara YM, White SDM, Baes M, Booth CM, Camps P, Navarro JF, Qu Y, Rahmati A, Sawala T, Thomas PA, Trayford J (2015) The EAGLE project: simulating the evolution and assembly of galaxies and their environments. MNRAS446(1):521-554, DOI 10.1093/ mnras/stu2058, 1407.7040

Scheuer PAG (1974) Models of extragalactic radio sources with a continuous energy supply from a central object. MNRAS166:513-528, DOI 10.1093/mnras/166.3.513 
Schiminovich D, van Gorkom JH, van der Hulst JM, Kasow S (1994) Discovery of Neutral Hydrogen Associated with the Diffuse Shells of NGC 5128 (Centaurus A). ApJLett423:L101, DOI 10.1086/ 187246

Schinnerer E, Eckart A, Tacconi LJ, Genzel R, Downes D (2000) Bars and Warps Traced by the Molecular Gas in the Seyfert 2 Galaxy NGC 1068. ApJ533(2):850-868, DOI 10.1086/308702, astro-ph/9911488

Schneider EE, Robertson BE (2015) CHOLLA: A New Massively Parallel Hydrodynamics Code for Astrophysical Simulation. ApJSupp.217(2):24, DOI 10.1088/0067-0049/217/2/24, 1410.4194

Schneider EE, Robertson BE (2017) Hydrodynamical Coupling of Mass and Momentum in Multiphase Galactic Winds. ApJ834(2):144, DOI 10.3847/1538-4357/834/2/144, 1607.01788

Schroetter I, Bouché N, Péroux C, Murphy MT, Contini T, Finley H (2015) The VLT SINFONI Mg II Program for Line Emitters (SIMPLE). II. Background Quasars Probing $Z \sim 1$ Galactic Winds. ApJ804(2):83, DOI 10.1088/0004-637X/804/2/83, 1502.07895

Schroetter I, Bouché N, Wendt M, Contini T, Finley H, Pelló R, Bacon R, Cantalupo S, Marino RA, Richard J, Lilly SJ, Schaye J, Soto K, Steinmetz M, Straka LA, Wisotzki L (2016) Muse Gas Flow and Wind (MEGAFLOW). I. First MUSE Results on Background Quasars. ApJ833(1):39, DOI 10. $3847 / 1538-4357 / 833 / 1 / 39,1605.03412$

Schroetter I, Bouché NF, Zabl J, Contini T, Wendt M, Schaye J, Mitchell P, Muzahid S, Marino RA, Bacon R, Lilly SJ, Richard J, Wisotzki L (2019) MusE GAs FLOw and Wind (MEGAFLOW) III: galactic wind properties using background quasars. arXiv e-prints arXiv:1907.09967, 1907.09967

Schulz NS, Cui W, Canizares CR, Marshall HL, Lee JC, Miller JM, Lewin WHG (2002) The First HighResolution X-Ray Spectrum of Cygnus X-1: Soft X-Ray Ionization and Absorption. ApJ565(2):11411149, DOI 10.1086/324482, astro-ph/0109236

Schulze A, Silverman JD, Daddi E, Rujopakarn W, Liu D, Schramm M, Mainieri V, Imanishi M, Hirschmann M, Jahnke K (2019) No signs of star formation being regulated in the most luminous quasars at $z \sim 2$ with ALMA. MNRAS488(1):1180-1198, DOI 10.1093/mnras/stz1746, 1906.04290

Schwartz CM, Martin CL (2004) A Keck/HIRES Study of Kinematics of the Cold Interstellar Medium in Dwarf Starburst Galaxies. ApJ610(1):201-212, DOI 10.1086/421546, astro-ph/0404148

Schweitzer M, Groves B, Netzer H, Lutz D, Sturm E, Contursi A, Genzel R, Tacconi LJ, Veilleux S, Kim DC, Rupke D, Baker AJ (2008) Extended Silicate Dust Emission in Palomar-Green QSOs. ApJ679(1):101-117, DOI 10.1086/587097, 0801.4637

Scoville N, Murchikova L, Walter F, Vlahakis C, Koda J, Vanden Bout P, Barnes J, Hernquist L, Sheth K, Yun M, Sanders D, Armus L, Cox P, Thompson T, Robertson B, Zschaechner L, Tacconi L, Torrey P, Hayward CC, Genzel R, Hopkins P, van der Werf P, Decarli R (2017) ALMA Resolves the Nuclear Disks of Arp 220. ApJ836(1):66, DOI 10.3847/1538-4357/836/1/66, 1605.09381

Seale JP, Looney LW, Wong T, Ott J, Klein U, Pineda JL (2012) The Life and Death of Dense Molecular Clumps in the Large Magellanic Cloud. ApJ751(1):42, DOI 10.1088/0004-637X/751/1/42, 1203. 4505

Sell PH, Tremonti CA, Hickox RC, Diamond-Stanic AM, Moustakas J, Coil A, Williams A, Rudnick G, Robaina A, Geach JE, Heinz S, Wilcots EM (2014) Massive compact galaxies with high-velocity outflows: morphological analysis and constraints on AGN activity. MNRAS441(4):3417-3443, DOI 10.1093/mnras/stu636, 1404.0677

Sellgren K, Werner MW, Dinerstein HL (1983) Extended near-infrared emission from visual reflection nebulae. ApJLett271:L13-L17, DOI 10.1086/184083

Seon Ki, Witt AN, Shinn Jh, Kim Ij (2014) Diffuse Extraplanar Dust in NGC 891. ApJLett785(1):L18, DOI 10.1088/2041-8205/785/1/L18, 1403.4905

Serkowski K, Mathewson DS, Ford VL (1975) Wavelength dependence of interstellar polarization and ratio of total to selective extinction. ApJ196:261-290, DOI 10.1086/153410

Shapley AE, Steidel CC, Pettini M, Adelberger KL (2003) Rest-Frame Ultraviolet Spectra of $z \sim 3$ Lyman Break Galaxies. ApJ588(1):65-89, DOI 10.1086/373922, astro-ph/0301230

Sharma P, Roy A, Nath BB, Shchekinov Y (2014) In a hot bubble: why does superbubble feedback work, but isolated supernovae do not? MNRAS443(4):3463-3476, DOI 10.1093/mnras/stu1307, 1402.6695

Sharp RG, Bland-Hawthorn J (2010) Three-Dimensional Integral Field Observations of 10 Galactic Winds. I. Extended Phase (gsim10 Myr) of Mass/Energy Injection Before the Wind Blows. ApJ711(2):818 852, DOI 10.1088/0004-637X/711/2/818, 1001.4315

Shih HY, Rupke DSN (2010) The Complex Structure of the Multi-phase Galactic Wind in a Starburst Merger. ApJ724(2):1430-1440, DOI 10.1088/0004-637X/724/2/1430, 1009.6020 
Shin J, Woo JH, Chung A, Baek J, Cho K, Kang D, Bae HJ (2019) Positive and Negative Feedback of AGN Outflows in NGC 5728. ApJ881(2):147, DOI 10.3847/1538-4357/ab2e72, 1907.00982

Shinn JH (2018) Candidate List of Edge-on Galaxies with Substantial Extraplanar Dust. ApJSupp.239(2):21, DOI 10.3847/1538-4365/aae3e5, 1809.08392

Shopbell PL, Bland-Hawthorn J (1998) The Asymmetric Wind in M82. ApJ493(1):129-153, DOI 10. 1086/305108, astro-ph/9708038

Shull JM, Tumlinson J, Jenkins EB, Moos HW, Rachford BL, Savage BD, Sembach KR, Snow TP, Sonneborn G, York DG, Blair WP, Green JC, Friedman SD, Sahnow DJ (2000) Far Ultraviolet Spectroscopic Explorer Observations of Diffuse Interstellar Molecular Hydrogen. ApJLett538(1):L73-L76, DOI $10.1086 / 312782$

Siebenmorgen R, Haas M, Krügel E, Schulz B (2005) Discovery of $10 \mu \mathrm{m}$ silicate emission in quasars Evidence of the AGN unification scheme. A\&A436(1):L5-L8, DOI 10.1051/0004-6361:200500109

Silich S, Tenorio-Tagle G, Muñoz-Tuñón C (2003) On the Rapidly Cooling Interior of Supergalactic Winds. ApJ590(2):791-796, DOI 10.1086/375133, astro-ph/0303235

Silk J (2013) Unleashing Positive Feedback: Linking the Rates of Star Formation, Supermassive Black Hole Accretion, and Outflows in Distant Galaxies. ApJ772(2):112, DOI 10.1088/0004-637X/772/2/ $112,1305.5840$

Silk J (2017) Feedback by Massive Black Holes in Gas-rich Dwarf Galaxies. ApJLett839(1):L13, DOI 10.3847/2041-8213/aa67da, 1703.08553

Silk J, Rees MJ (1998) Quasars and galaxy formation. A\&A331:L1-L4, astro-ph/9801013

Silvia DW, Smith BD, Shull JM (2010) Numerical Simulations of Supernova Dust Destruction. I. Cloudcrushing and Post-processed Grain Sputtering. ApJ715(2):1575-1590, DOI 10.1088/0004-637X/715/ $2 / 1575,1001.4793$

Simons RC, Kassin SA, Weiner BJ, Heckman TM, Lee JC, Lotz JM, Peth M, Tchernyshyov K (2015) A transition mass in the local Tully-Fisher relation. MNRAS452(1):986-997, DOI 10.1093/mnras/ stv1298, 1506.04144

Skinner MA, Ostriker EC (2015) Numerical Simulations of Turbulent Molecular Clouds Regulated by Reprocessed Radiation Feedback from Nascent Super Star Clusters. ApJ809(2):187, DOI 10.1088/ 0004-637X/809/2/187, 1507.06366

Slavin JD, Jones AP, Tielens AGGM (2004) Shock Processing of Large Grains in the Interstellar Medium. ApJ614(2):796-806, DOI 10.1086/423834

Slavin JD, Dwek E, Jones AP (2015) Destruction of Interstellar Dust in Evolving Supernova Remnant Shock Waves. ApJ803(1):7, DOI 10.1088/0004-637X/803/1/7, 1502.00929

Smith MWL, Eales SA, De Looze I, Baes M, Bendo GJ, Bianchi S, Boquien M, Boselli A, Buat V, Ciesla L, Clemens M, Clements DL, Cooray AR, Cortese L, Davies JI, Fritz J, Gomez HL, Hughes TM, Karczewski OŁ, Lu N, Oliver SJ, Remy-Ruyer A, Spinoglio L, Viaene S (2016) Far-reaching dust distribution in galaxy discs. MNRAS462(1):331-344, DOI 10.1093/mnras/stw1611, 1607.01020

Smith PS, Schmidt GD, Allen RG, Angel JRP (1995) The Polarization and Ultraviolet Spectrum of Markarian 231. ApJ444:146, DOI 10.1086/175589

Smith RN, Tombesi F, Veilleux S, Lohfink AM, Luminari A (2019) Discovery of an X-ray Quasar Wind Driving the Cold Gas Outflow in the Ultraluminous Infrared Galaxy IRAS F05189-2524. arXiv eprints arXiv: $1910.14583,1910.14583$

Socrates A, Davis SW, Ramirez-Ruiz E (2008) The Eddington Limit in Cosmic Rays: An Explanation for the Observed Faintness of Starbursting Galaxies. ApJ687(1):202-215, DOI 10.1086/590046, astro-ph/0609796

Sofia UJ, Lauroesch JT, Meyer DM, Cartledge SIB (2004) Interstellar Carbon in Translucent Sight Lines. ApJ605(1):272-277, DOI 10.1086/382592, astro-ph/0401510

Sofia UJ, Parvathi VS, Babu BRS, Murthy J (2011) Determining Interstellar Carbon Abundances from Strong-line Transitions. AJ141(1):22, DOI 10.1088/0004-6256/141/1/22

Sofue Y, Handa T (1984) A radio lobe over the galactic centre. Nature310(5978):568-569, DOI 10.1038/ $310568 \mathrm{a} 0$

Sokal KR, Johnson KE, Indebetouw R, Massey P (2016) The Prevalence and Impact of Wolf-Rayet Stars in Emerging Massive Star Clusters. ApJ826(2):194, DOI 10.3847/0004-637X/826/2/194, 1605.08044

Solomon PM, Vanden Bout PA (2005) Molecular Gas at High Redshift. ARA\&A43(1):677-725, DOI 10.1146/annurev.astro.43.051804.102221, astro-ph/0508481

Som D, Kulkarni VP, Meiring J, York DG, Péroux C, Lauroesch JT, Aller MC, Khare P (2015) Hubble Space Telescope Observations of Sub-damped Ly $\alpha$ Absorbers at z \&lt; 0.5, and Implications for Galaxy Chemical Evolution. ApJ806(1):25, DOI 10.1088/0004-637X/806/1/25, 1502 .01989 
Soma T, Sakai N, Watanabe Y, Yamamoto S (2015) Methanol in the Starless Core, Taurus Molecular Cloud-1. ApJ802(2):74, DOI 10.1088/0004-637X/802/2/74

Somerville RS, Popping G, Trager SC (2015) Star formation in semi-analytic galaxy formation models with multiphase gas. MNRAS453(4):4337-4367, DOI 10.1093/mnras/stv1877, 1503.00755

Sormani MC, Barnes AT (2019) Mass inflow rate into the Central Molecular Zone: observational determination and evidence of episodic accretion. MNRAS484(1):1213-1219, DOI 10.1093/mnras/stz046, 1901.00867

Sormani MC, Binney J, Magorrian J (2015) Gas flow in barred potentials. MNRAS449(3):2421-2435, DOI 10.1093/mnras/stv441, 1502.02740

Sparre M, Pfrommer C, Vogelsberger M (2019) The physics of multiphase gas flows: fragmentation of a radiatively cooling gas cloud in a hot wind. MNRAS482(4):5401-5421, DOI 10.1093/mnras/sty3063, 1807.07971

Spilker JS, Aravena M, Béthermin M, Chapman SC, Chen CC, Cunningham DJM, De Breuck C, Dong C, Gonzalez AH, Hayward CC, Hezaveh YD, Litke KC, Ma J, Malkan M, Marrone DP, Miller TB, Morningstar WR, Narayanan D, Phadke KA, Sreevani J, Stark AA, Vieira JD, Weiß A (2018) Fast molecular outflow from a dusty star-forming galaxy in the early Universe. Science 361(6406):10161019, DOI 10.1126/science.aap8900, 1809.01676

Spilker JS, Bezanson R, Weiner BJ, Whitaker KE, Williams CC (2019) Evidence for Inside-Out Galaxy Growth and Quenching of a $z \sim 2$ Compact Galaxy from High-Resolution Molecular Gas Imaging. arXiv e-prints arXiv: $1908.02294,1908.02294$

Spitzer J L (1968) Diffuse matter in space. Interscience Tracts on Physics and Astronomy 28

Spitzer J Lyman, Cochran WD (1973) Rotational Excitation of Interstellar H_\{2\}. ApJLett186:L23, DOI $10.1086 / 181349$

Spitzer L (1978) Physical processes in the interstellar medium. DOI 10.1002/9783527617722

Spoon HWW, Tielens AGGM, Armus L, Sloan GC, Sargent B, Cami J, Charmandaris V, Houck JR, Soifer BT (2006) The Detection of Crystalline Silicates in Ultraluminous Infrared Galaxies. ApJ638(2):759 765, DOI 10.1086/498566, astro-ph/0509859

Spoon HWW, Marshall JA, Houck JR, Elitzur M, Hao L, Armus L, Brandl BR, Charmandaris V (2007) Mid-Infrared Galaxy Classification Based on Silicate Obscuration and PAH Equivalent Width. ApJLett654(1):L49-L52, DOI 10.1086/511268, astro-ph/0611918

Spoon HWW, Farrah D, Lebouteiller V, González-Alfonso E, Bernard-Salas J, Urrutia T, Rigopoulou D, Westmoquette MS, Smith HA, Afonso J, Pearson C, Cormier D, Efstathiou A, Borys C, Verma A, Etxaluze M, Clements DL (2013) Diagnostics of AGN-Driven Molecular Outflows in ULIRGs from Herschel-PACS Observations of $\mathrm{OH}$ at $119 \mu \mathrm{m}$. ApJ775(2):127, DOI 10.1088/0004-637X/775/2/127, 1307.6224

Stacey GJ, Townes CH, Poglitsch A, Madden SC, Jackson JM, Herrmann F, Genzel R, Geis N (1991) The Optical Depth of the 158 Micron [ $12 \mathrm{C}$ ii] Line: Detection of the F $=10$ [ $13 \mathrm{C}$ ii] Hyperfine-Structure Component. ApJLett382:L37, DOI 10.1086/186208

Stalevski M, Tristram KRW, Asmus D (2019) Dissecting the active galactic nucleus in Circinus - II. A thin dusty disc and a polar outflow on parsec scales. MNRAS484(3):3334-3355, DOI 10.1093/mnras/ stz220, 1901.05488

Stanimirović S, Staveley-Smith L, Jones PA (2004) A New Look at the Kinematics of Neutral Hydrogen in the Small Magellanic Cloud. ApJ604(1):176-186, DOI 10.1086/381869, astro-ph/0312223

Stanley F, Jolly JB, König S, Knudsen KK (2019) A spectral stacking analysis to search for faint outflow signatures in $z \sim 6$ quasars. arXiv e-prints arXiv:1908.11395, 1908.11395

Stark DP, Auger M, Belokurov V, Jones T, Robertson B, Ellis RS, Sand DJ, Moiseev A, Eagle W, Myers $\mathrm{T}$ (2013) The CASSOWARY spectroscopy survey: a new sample of gravitationally lensed galaxies in SDSS. MNRAS436(2):1040-1056, DOI 10.1093/mnras/stt1624, 1302.2663

Staveley-Smith L, Kim S, Calabretta MR, Haynes RF, Kesteven MJ (2003) A new look at the large-scale HI structure of the Large Magellanic Cloud. MNRAS339(1):87-104, DOI 10.1046/j.1365-8711.2003. 06146.x, astro-ph/0210501

Steidel CC, Erb DK, Shapley AE, Pettini M, Reddy N, Bogosavljević M, Rudie GC, Rakic O (2010) The Structure and Kinematics of the Circumgalactic Medium from Far-ultraviolet Spectra of $z \sim 2-3$ Galaxies. ApJ717(1):289-322, DOI 10.1088/0004-637X/717/1/289, 1003.0679

Steidel CC, Bogosavljević M, Shapley AE, Kollmeier JA, Reddy NA, Erb DK, Pettini M (2011) Diffuse Ly $\alpha$ Emitting Halos: A Generic Property of High-redshift Star-forming Galaxies. ApJ736(2):160, DOI 10.1088/0004-637X/736/2/160, 1101.2204

Stern J, Hennawi JF, Prochaska JX, Werk JK (2016) A Universal Density Structure for Circumgalactic 
Gas. ApJ830(2):87, DOI 10.3847/0004-637X/830/2/87, 1604.02168

Sternberg A, Dalgarno A (1995) Chemistry in Dense Photon-dominated Regions. ApJSupp.99:565, DOI $10.1086 / 192198$

Sternberg A, Genzel R, Tacconi L (1994) HCN and CO in the Nucleus of NGC 1068. ApJLett436:L131, DOI $10.1086 / 187650$

Stocke JT, Case J, Donahue M, Shull JM, Snow TP (1991) New Observations of the Gas Cloud Associated with the Quasar-Galaxy Pair 3C 232/NGC 3067. ApJ374:72, DOI 10.1086/170097

Stokes GM (1978) Interstellar titanium. ApJSupp.36:115-141, DOI 10.1086/190493

Stone M, Veilleux S, Meléndez M, Sturm E, Graciá-Carpio J, González-Alfonso E (2016) The Search for Molecular Outflows in Local Volume AGNs with Herschel-PACS. ApJ826(2):111, DOI 10.3847/ 0004-637X/826/2/111, 1605.06512

Stone M, Veilleux S, González-Alfonso E, Spoon H, Sturm E (2018) Constraints on the OH-to-H Abundance Ratio in Infrared-bright Galaxies Derived from the Strength of the $\mathrm{OH} 35 \mu \mathrm{m}$ Absorption Feature. ApJ853(2):132, DOI 10.3847/1538-4357/aaa3df

Storey JWV, Watson DM, Townes CH (1981) Detection of interstellar OH in the far-infrared. ApJLett244:L27-L30, DOI 10.1086/183472

Strickland DK, Heckman TM (2009) Supernova Feedback Efficiency and Mass Loading in the Starburst and Galactic Superwind Exemplar M82. ApJ697(2):2030-2056, DOI 10.1088/0004-637X/697/ 2/2030,0903.4175

Strickland DK, Ponman TJ, Stevens IR (1997) ROSAT observations of the galactic wind in M 82. A\&A320:378-394, astro-ph/9608064

Strickland DK, Heckman TM, Weaver KA, Dahlem M (2000) Chandra Observations of NGC 253: New Insights into the Nature of Starburst-driven Superwinds. AJ120(6):2965-2974, DOI 10.1086/316846, astro-ph/0008182

Strickland DK, Heckman TM, Weaver KA, Hoopes CG, Dahlem M (2002) Chandra Observations of NGC 253. II. On the Origin of Diffuse X-Ray Emission in the Halos of Starburst Galaxies. ApJ568(2):689716, DOI 10.1086/338889, astro-ph/0111511

Struve C, Oosterloo TA, Morganti R, Saripalli L (2010) Centaurus A: morphology and kinematics of the atomic hydrogen. A\&A515:A67, DOI 10.1051/0004-6361/201014355, 1003.3250

Sturm E, Lutz D, Tran D, Feuchtgruber H, Genzel R, Kunze D, Moorwood AFM, Thornley MD (2000) ISO-SWS spectra of galaxies: Continuum and features. A\&A358:481-493, astro-ph/0002195

Sturm E, Schweitzer M, Lutz D, Contursi A, Genzel R, Lehnert MD, Tacconi LJ, Veilleux S, Rupke DS, Kim DC, Sternberg A, Maoz D, Lord S, Mazzarella J, Sanders DB (2005) Silicate Emissions in Active Galaxies: From LINERs to QSOs. ApJLett629(1):L21-L23, DOI 10.1086/444359, astro-ph/0506716

Sturm E, Rupke D, Contursi A, Kim DC, Lutz D, Netzer H, Veilleux S, Genzel R, Lehnert M, Tacconi LJ, Maoz D, Mazzarella J, Lord S, Sanders D, Sternberg A (2006) Mid-Infrared Diagnostics of LINERS. ApJLett653(1):L13-L16, DOI 10.1086/510381, astro-ph/0610772

Sturm E, González-Alfonso E, Veilleux S, Fischer J, Graciá-Carpio J, Hailey-Dunsheath S, Contursi A, Poglitsch A, Sternberg A, Davies R, Genzel R, Lutz D, Tacconi L, Verma A, Maiolino R, de Jong JA (2011) Massive Molecular Outflows and Negative Feedback in ULIRGs Observed by Herschel-PACS. ApJLett733(1):L16, DOI 10.1088/2041-8205/733/1/L16, 1105. 1731

Sturm R, Haberl F (2014) The diffuse X-ray emission of the Small Magellanic Cloud. In: The X-ray Universe 2014, p 191

Su M, Slatyer TR, Finkbeiner DP (2010) Giant Gamma-ray Bubbles from Fermi-LAT: Active Galactic Nucleus Activity or Bipolar Galactic Wind? ApJ724(2):1044-1082, DOI 10.1088/0004-637X/724/2/ $1044,1005.5480$

Sugahara Y, Ouchi M, Lin L, Martin CL, Ono Y, Harikane Y, Shibuya T, Yan R (2017) Evolution of Galactic Outflows at $z \sim 0-2$ Revealed with SDSS, DEEP2, and Keck Spectra. ApJ850(1):51, DOI 10.3847/1538-4357/aa956d, 1703.01885

Sugahara Y, Ouchi M, Harikane Y, Bouché N, Mitchell PD, Blaizot J (2019) Fast Outflows Identified in Early Star-Forming Galaxies at $z=5-6$. arXiv e-prints arXiv:1904.03106, 1904.03106

Sunyaev RA, Markevitch M, Pavlinsky M (1993) The Center of the Galaxy in the Recent Past: A View from GRANAT. ApJ407:606, DOI 10.1086/172542

Tacchella S, Carollo CM, Renzini A, Schreiber NMF, Lang P, Wuyts S, Cresci G, Dekel A, Genzel R, Lilly SJ, Mancini C, Newman S, Onodera M, Shapley A, Tacconi L, Woo J, Zamorani G (2015) Evidence for mature bulges and an inside-out quenching phase 3 billion years after the Big Bang. Science 348(6232):314-317, DOI 10.1126/science.1261094, 1504.04021 
Tacchella S, Dekel A, Carollo CM, Ceverino D, DeGraf C, Lapiner S, Mand elker N, Primack JR (2016) Evolution of density profiles in high-z galaxies: compaction and quenching inside-out. MNRAS458(1):242-263, DOI 10.1093/mnras/stw303, 1509.00017

Tacconi LJ, Genzel R, Blietz M, Cameron M, Harris AI, Madden S (1994) The Nature of the Dense Obscuring Material in the Nucleus of NGC 1068. ApJLett426:L77, DOI 10.1086/187344

Tacconi-Garman LE, Sturm E, Lehnert M, Lutz D, Davies RI, Moorwood AFM (2005) PAH emission variations within the resolved starbursts of NGC 253 and NGC 1808. A\&A432(1):91-103, DOI 10.1051/0004-6361:20035738, astro-ph/0411272

Tadhunter C, Morganti R, Rose M, Oonk JBR, Oosterloo T (2014) Jet acceleration of the fast molecular outflows in the Seyfert galaxy IC 5063. Nature511(7510):440-443, DOI 10.1038/nature13520, 1407. 1332

Talia M, Brusa M, Cimatti A, Lemaux BC, Amorin R, Bardelli S, Cassarà LP, Cucciati O, Garilli B, Grazian A, Guaita L, Hathi NP, Koekemoer A, Le Fèvre O, Maccagni D, Nakajima K, Pentericci L, Pforr J, Schaerer D, Vanzella E, Vergani D, Zamorani G, Zucca E (2017) AGN-enhanced outflows of low-ionization gas in star-forming galaxies at 1.7 \&lt; z \&lt; 4.6*. MNRAS471(4):4527-4540, DOI 10.1093/mnras/stx1788, 1611.05884

Tanaka K, Nagai M, Kamegai K, Iino T, Sakai T (2018) HCN J = 4-3, HNC J = 1-0, $\mathrm{H}^{13} \mathrm{CN} J=1-0$, and $\mathrm{HC}_{3} \mathrm{~N} \mathrm{~J}=10-9$ Maps of the Galactic Center Region. I. Spatially Resolved Measurements of Physical Conditions and Chemical Composition. ApJSupp.236(2):40, DOI 10.3847/1538-4365/aab9a5, 1804. 00666

Tanner R, Cecil G, Heitsch F (2016) Starburst-driven Galactic Superbubbles Radiating to $10 \mathrm{~K}$. ApJ821(1):7, DOI 10.3847/0004-637X/821/1/7, 1511.05165

Tanner R, Cecil G, Heitsch F (2017) Scaling Relations of Starburst-driven Galactic Winds. ApJ843(2):137, DOI 10.3847/1538-4357/aa78a8, 1608.05342

Tecza M, Thatte N, Maiolino R (2001) Probing the heart of an active galactic nucleus: NGC 1068. In: Schilizzi RT (ed) Galaxies and their Constituents at the Highest Angular Resolutions, IAU Symposium, vol 205, p 216

Teimoorinia H, Bluck AFL, Ellison SL (2016) An artificial neural network approach for ranking quenching parameters in central galaxies. MNRAS457(2):2086-2106, DOI 10.1093/mnras/stw036, 1601. 01258

Temim T, Dwek E, Tchernyshyov K, Boyer ML, Meixner M, Gall C, Roman-Duval J (2015) Dust Destruction Rates and Lifetimes in the Magellanic Clouds. ApJ799(2):158, DOI 10.1088/0004-637X/ $799 / 2 / 158,1411.4574$

Temple MJ, Banerji M, Hewett PC, Coatman L, Maddox N, Peroux C (2019) [O III] Emission line properties in a new sample of heavily reddened quasars at z \&gt; 2. MNRAS487(2):2594-2613, DOI $10.1093 / \mathrm{mnras} / \mathrm{stz} 1420,1905.08198$

Teng SH, Veilleux S, Baker AJ (2013) Green Bank Telescope Detection of Polarization-dependent H I Absorption and H I Outflows in Local ULIRGs and Quasars. ApJ765(2):95, DOI 10.1088/0004-637X/ $765 / 2 / 95,1301.5642$

Teng SH, Brandt WN, Harrison FA, Luo B, Alexander DM, Bauer FE, Boggs SE, Christensen FE, Comastri A, Craig WW, Fabian AC, Farrah D, Fiore F, Gandhi P, Grefenstette BW, Hailey CJ, Hickox RC, Madsen KK, Ptak AF, Rigby JR, Risaliti G, Saez C, Stern D, Veilleux S, Walton DJ, Wik DR, Zhang WW (2014) NuSTAR Reveals an Intrinsically X-Ray Weak Broad Absorption Line Quasar in the Ultraluminous Infrared Galaxy Markarian 231. ApJ785(1):19, DOI 10.1088/0004-637X/785/1/19, 1402.4811

Teplitz HI, Armus L, Soifer BT, Charmand aris V, Marshall JA, Spoon H, Lawrence C, Hao L, Higdon S, Wu Y, Lacy M, Eisenhardt PR, Herter T, Houck JR (2006) Silicate Emission in the Spitzer IRS Spectrum of FSC 10214+4724. ApJLett638(1):L1-L4, DOI 10.1086/500791, astro-ph/0601061

Thom C, Tumlinson J, Werk JK, Prochaska JX, Oppenheimer BD, Peeples MS, Tripp TM, Katz NS, O'Meara JM, Ford AB, Davé R, Sembach KR, Weinberg DH (2012) Not Dead Yet: Cool Circumgalactic Gas in the Halos of Early-type Galaxies. ApJLett758(2):L41, DOI 10.1088/2041-8205/758/ 2/L41, 1209. 5442

Thompson TA, Krumholz MR (2016) Sub-Eddington star-forming regions are super-Eddington momentum-driven outflows from supersonic turbulence. MNRAS455(1):334-342, DOI 10.1093/ mnras/stv2331, 1411.1769

Thompson TA, Quataert E, Murray N (2005) Radiation Pressure-supported Starburst Disks and Active Galactic Nucleus Fueling. ApJ630(1):167-185, DOI 10.1086/431923, astro-ph/0503027

Thompson TA, Fabian AC, Quataert E, Murray N (2015) Dynamics of dusty radiation-pressure- 
driven shells and clouds: fast outflows from galaxies, star clusters, massive stars, and AGN. MNRAS449(1):147-161, DOI 10.1093/mnras/stv246, 1406.5206

Thompson TA, Quataert E, Zhang D, Weinberg DH (2016) An origin for multiphase gas in galactic winds and haloes. MNRAS455(2):1830-1844, DOI 10.1093/mnras/stv2428, 1507.04362

Thompson TWJ, Howk JC, Savage BD (2004) Hubble Space Telescope Imaging of Extraplanar Dust Structures in the Edge-On Spiral NGC 4217. AJ128(2):662-673, DOI 10.1086/422485

Thorne KS (1974) Disk-Accretion onto a Black Hole. II. Evolution of the Hole. ApJ191:507-520, DOI $10.1086 / 152991$

Tollet É, Cattaneo A, Macciò AV, Dutton AA, Kang X (2019) NIHAO XIX: how supernova feedback shapes the galaxy baryon cycle. MNRAS485(2):2511-2531, DOI 10.1093/mnras/stz545, 1902 . 03888

Tombesi F, Meléndez M, Veilleux S, Reeves JN, González-Alfonso E, Reynolds CS (2015) Wind from the black-hole accretion disk driving a molecular outflow in an active galaxy. Nature519(7544):436-438, DOI 10.1038/nature14261, 1501.07664

Tombesi F, Veilleux S, Meléndez M, Lohfink A, Reeves JN, Piconcelli E, Fiore F, Feruglio C (2017) NuSTAR View of the Black Hole Wind in the Galaxy Merger IRAS F11119+3257. ApJ850(2):151, DOI 10.3847/1538-4357/aa9579, 1710.07485

Treister E, Messias H, Privon GC, Nagar N, Medling AM, U V, Bauer FE, Cicone C, Barcos Munoz L, Evans AS, Muller-Sanchez F, Comerford JM, Armus L, Chang C, Koss M, Venturi G, Schawinski K, Casey C, Urry CM, Sanders DB, Scoville N, Sheth K (2020) The Molecular Gas in the NGC 6240 Merging Galaxy System at the Highest Spatial Resolution. arXiv e-prints arXiv:2001.00601, 2001.00601

Tremblay GR, Combes F, Oonk JBR, Russell HR, McDonald MA, Gaspari M, Husemann B, Nulsen PEJ, McNamara BR, Hamer SL, O’ Dea CP, Baum SA, Davis TA, Donahue M, Voit GM, Edge AC, Blanton EL, Bremer MN, Bulbul E, Clarke TE, David LP, Edwards LOV, Eggerman D, Fabian AC, Forman W, Jones C, Kerman N, Kraft RP, Li Y, Powell M, Randall SW, Salomé P, Simionescu A, Su Y, Sun M, Urry CM, Vantyghem AN, Wilkes BJ, ZuHone JA (2018) A Galaxy-scale Fountain of Cold Molecular Gas Pumped by a Black Hole. ApJ865(1):13, DOI 10.3847/1538-4357/aad6dd, 1808.00473

Tremonti CA, Moustakas J, Diamond-Stanic AaM (2007) The Discovery of $1000 \mathrm{~km} \mathrm{~s}^{-1}$ Outflows in Massive Poststarburst Galaxies at z=0.6. ApJLett663(2):L77-L80, DOI 10.1086/520083, 0706.0527

Tristram KRW, Burtscher L, Jaffe W, Meisenheimer K, Hönig SF, Kishimoto M, Schartmann M, Weigelt $\mathrm{G}$ (2014) The dusty torus in the Circinus galaxy: a dense disk and the torus funnel. A\&A563:A82, DOI 10.1051/0004-6361/201322698, 1312.4534

Trump JR, Hall PB, Reichard TA, Richards GT, Schneider DP, Vand en Berk DE, Knapp GR, Anderson SF, Fan X, Brinkman J, Kleinman SJ, Nitta A (2006) A Catalog of Broad Absorption Line Quasars from the Sloan Digital Sky Survey Third Data Release. ApJSupp.165(1):1-18, DOI 10.1086/503834, astro-ph/0603070

Trussler J, Maiolino R, Maraston C, Peng Y, Thomas D, Goddard D, Lian J (2018) Starvation as the primary quenching mechanism in galaxies. arXiv e-prints arXiv:1811.09283, 1811.09283

Tsai AL, Matsushita S, Kong AKH, Matsumoto H, Kohno K (2012) First Detection of a Subkiloparsec Scale Molecular Outflow in the Starburst Galaxy NGC 3628. ApJ752(1):38, DOI 10.1088/ 0004-637X/752/1/38, 1204.3414

Tsang BTH, Milosavljević M (2015) Radiation pressure driving of a dusty atmosphere. MNRAS453(1):1108-1120, DOI 10.1093/mnras/stv1707, 1506.05121

Tumlinson J, Peeples MS, Werk JK (2017) The Circumgalactic Medium. ARA\&A55(1):389-432, DOI 10.1146/annurev-astro-091916-055240, 1709.09180

Tunnard R, Greve TR, Garcia-Burillo S, Graciá Carpio J, Fischer J, Fuente A, González-Alfonso E, Hailey-Dunsheath S, Neri R, Sturm E, Usero A, Planesas P (2015) Chemically Distinct Nuclei and Outflowing Shocked Molecular Gas in Arp 220. ApJ800(1):25, DOI 10.1088/0004-637X/800/1/25, 1412.3114

Turner BE (1985) VLA observations of OH in galaxies. I. NGC 253 and its nuclear plume. ApJ299:312_ 333, DOI 10.1086/163702

Uhlig M, Pfrommer C, Sharma M, Nath BB, Enßlin TA, Springel V (2012) Galactic winds driven by cosmic ray streaming. MNRAS423(3):2374-2396, DOI 10.1111/j.1365-2966.2012.21045.x, 1203. 1038

Usero A, García-Burillo S, Fuente A, Martín-Pintado J, Rodríguez-Fernández NJ (2004) Molecular gas chemistry in AGN. I. The IRAM $30 \mathrm{~m}$ survey of NGC 1068. A\&A419:897-912, DOI 10.1051/ 0004-6361:20035774, astro-ph/0402556 
Valiante R, Schneider R, Maiolino R, Salvadori S, Bianchi S (2012) Quasar feedback in the early Universe: the case of SDSS J1148+5251. MNRAS427(1):L60-L64, DOI 10.1111/j.1745-3933.2012.01345.x, 1205.3488

van der Tak FFS, Black JH, Schöier FL, Jansen DJ, van Dishoeck EF (2007) A computer program for fast non-LTE analysis of interstellar line spectra. With diagnostic plots to interpret observed line intensity ratios. A\&A468(2):627-635, DOI 10.1051/0004-6361:20066820, 0704.0155

van der Werf PP, Berciano Alba A, Spaans M, Loenen AF, Meijerink R, Riechers DA, Cox P, Weiß A, Walter F (2011) Water Vapor Emission Reveals a Highly Obscured, Star-forming Nuclear Region in the QSO Host Galaxy APM 08279+5255 at $\mathrm{z}=3.9$. ApJLett741(2):L38, DOI 10.1088/2041-8205/ $741 / 2 / \mathrm{L} 38,1106.4825$

van Marle AJ, Keppens R (2012) Multi-dimensional models of circumstellar shells around evolved massive stars. A\&A547:A3, DOI 10.1051/0004-6361/201218957, 1209. 4496

Vantyghem AN, McNamara BR, Russell HR, Edge AC, Nulsen PEJ, Combes F, Fabian AC, McDonald M, Salomé P (2018) Molecular Gas Filaments and Star-forming Knots Beneath an X-Ray Cavity in RXC J1504-0248. ApJ863(2):193, DOI 10.3847/1538-4357/aad2e0, 1807.04331

Vantyghem AN, McNamara BR, Russell HR, Edge AC, Nulsen PEJ, Combes F, Fabian AC, McDonald M, Salomé P (2019) An Enormous Molecular Gas Flow in the RX J0821+0752 Galaxy Cluster. ApJ870(2):57, DOI 10.3847/1538-4357/aaf1b4, 1811.09653

Vayner A, Wright SA, Murray N, Armus L, Larkin JE, Mieda E (2017) Galactic-scale Feedback Observed in the 3C 298 Quasar Host Galaxy. ApJ851(2):126, DOI 10.3847/1538-4357/aa9c42, 1709.03510

Veilleux S (1991) A Study of the Structure and Kinematics of the Narrow-Line Region in Seyfert Galaxies. I. Atlas of Line Profiles. ApJSupp.75:357, DOI 10.1086/191534

Veilleux S, Cecil G, Bland-Hawthorn J, Tully RB, Filippenko AV, Sargent WLW (1994) The Nuclear Superbubble of NGC 3079. ApJ433:48, DOI 10.1086/174624

Veilleux S, Kim DC, Sanders DB, Mazzarella JM, Soifer BT (1995) Optical Spectroscopy of Luminous Infrared Galaxies. II. Analysis of the Nuclear and Long-Slit Data. ApJSupp.98:171, DOI 10.1086/ 192158

Veilleux S, Cecil G, Bland-Hawthorn J (2005) Galactic Winds. ARA\&A43(1):769-826, DOI 10.1146/ annurev.astro.43.072103.150610, astro-ph/0504435

Veilleux S, Kim DC, Rupke DSN, Peng CY, Tacconi LJ, Genzel R, Lutz D, Sturm E, Contursi A, Schweitzer M, Dasyra KM, Ho LC, Sanders DB, Burkert A (2009a) A Deep Hubble Space Telescope H-Band Imaging Survey of Massive Gas-Rich Mergers. II. The QUEST QSOs. ApJ701(1):587-606, DOI 10.1088/0004-637X/701/1/587, 0906.3157

Veilleux S, Rupke DSN, Swaters R (2009b) Warm Molecular Hydrogen in the Galactic Wind of M82. ApJLett700(2):L149-L153, DOI 10.1088/0004-637X/700/2/L149, 0907.1422

Veilleux S, Meléndez M, Sturm E, Gracia-Carpio J, Fischer J, González-Alfonso E, Contursi A, Lutz D, Poglitsch A, Davies R, Genzel R, Tacconi L, de Jong JA, Sternberg A, Netzer H, Hailey-Dunsheath S, Verma A, Rupke DSN, Maiolino R, Teng SH, Polisensky E (2013a) Fast Molecular Outflows in Luminous Galaxy Mergers: Evidence for Quasar Feedback from Herschel. ApJ776(1):27, DOI $10.1088 / 0004-637 \mathrm{X} / 776 / 1 / 27,1308.3139$

Veilleux S, Trippe M, Hamann F, Rupke DSN, Tripp TM, Netzer H, Lutz D, Sembach KR, Krug H, Teng SH, Genzel R, Maiolino R, Sturm E, Tacconi L (2013b) The Surprising Absence of Absorption in the Far-ultraviolet Spectrum of Mrk 231. ApJ764(1):15, DOI 10.1088/0004-637X/764/1/15, 1212.2401

Veilleux S, Teng SH, Rupke DSN, Maiolino R, Sturm E (2014) Half-megasecond Chandra Spectral Imaging of the Hot Circumgalactic Nebula around Quasar Mrk 231. ApJ790(2):116, DOI $10.1088 / 0004-637 X / 790 / 2 / 116,1405.4833$

Veilleux S, Meléndez M, Tripp TM, Hamann F, Rupke DSN (2016) The Complete Ultraviolet Spectrum of the Archetypal "Wind-dominated" Quasar Mrk 231: Absorption and Emission from a High-speed Dusty Nuclear Outflow. ApJ825(1):42, DOI 10.3847/0004-637X/825/1/42, 1605.00665

Veilleux S, Bolatto A, Tombesi F, Meléndez M, Sturm E, González-Alfonso E, Fischer J, Rupke DSN (2017) Quasar Feedback in the Ultraluminous Infrared Galaxy F11119+3257: Connecting the Accretion Disk Wind with the Large-scale Molecular Outflow. ApJ843(1):18, DOI 10.3847/1538-4357/ aa767d, 1706.00443

Verhamme A, Schaerer D, Atek H, Tapken C (2008) 3D Ly $\alpha$ radiation transfer. III. Constraints on gas and stellar properties of $z \sim 3$ Lyman break galaxies (LBG) and implications for high-z LBGs and Ly $\alpha$ emitters. A\&A491(1):89-111, DOI 10.1051/0004-6361:200809648, 0805. 3601

Vermot P, Clénet Y, Gratadour D (2019) New insights on the central stellar population and gas outflow in NGC 1068 from YJH spectroscopy with SPHERE/VLT. arXiv e-prints arXiv:1905.09208, 1905. 
09208

Vijayan A, Nath BB, Sharma P, Shchekinov Y (2019) Radio halos of star forming galaxies. arXiv e-prints arXiv: $1911.07870,1911.07870$

Viti S (2016) Astrochemistry in external galaxies: how to use molecules as probes of their physical conditions. In: Jablonka P, André P, van der Tak F (eds) From Interstellar Clouds to Star-Forming Galaxies: Universal Processes?, IAU Symposium, vol 315, pp 17-25, DOI 10.1017/S1743921316007195, 1603.09105

Viti S, García-Burillo S, Fuente A, Hunt LK, Usero A, Henkel C, Eckart A, Martin S, Spaans M, Muller S, Combes F, Krips M, Schinnerer E, Casasola V, Costagliola F, Marquez I, Planesas P, van der Werf PP, Aalto S, Baker AJ, Boone F, Tacconi LJ (2014) Molecular line emission in NGC 1068 imaged with ALMA. II. The chemistry of the dense molecular gas. A\&A570:A28, DOI 10.1051/0004-6361/ 201424116, 1407.4940

Vogelsberger M, Genel S, Springel V, Torrey P, Sijacki D, Xu D, Snyder G, Nelson D, Hernquist L (2014) Introducing the Illustris Project: simulating the coevolution of dark and visible matter in the Universe. MNRAS444(2):1518-1547, DOI 10.1093/mnras/stu1536, 1405.2921

Wagner AY, Bicknell GV, Umemura M (2012) Driving Outflows with Relativistic Jets and the Dependence of Active Galactic Nucleus Feedback Efficiency on Interstellar Medium Inhomogeneity. ApJ757(2):136, DOI 10.1088/0004-637X/757/2/136, 1205.0542

Wagner AY, Umemura M, Bicknell GV (2013) Ultrafast Outflows: Galaxy-scale Active Galactic Nucleus Feedback. ApJLett763(1):L18, DOI 10.1088/2041-8205/763/1/L18, 1211.5851

Wakelam V, Bron E, Cazaux S, Dulieu F, Gry C, Guillard P, Habart E, Hornekær L, Morisset S, Nyman G, Pirronello V, Price SD, Valdivia V, Vidali G, Watanabe N (2017) $\mathrm{H}_{2}$ formation on interstellar dust grains: The viewpoints of theory, experiments, models and observations. Molecular Astrophysics 9:1-36, DOI 10.1016/j.molap.2017.11.001, 1711.10568

Walch S, Naab T (2015) The energy and momentum input of supernova explosions in structured and ionized molecular clouds. MNRAS451(3):2757-2771, DOI 10.1093/mnras/stv1155, 1410.0011

Walter F, Weiss A, Scoville N (2002) Molecular Gas in M82: Resolving the Outflow and Streamers. ApJLett580(1):L21-L25, DOI 10.1086/345287, astro-ph/0210602

Walter F, Weiß A, Downes D, Decarli R, Henkel C (2011) A Survey of Atomic Carbon at High Redshift. ApJ730(1):18, DOI 10.1088/0004-637X/730/1/18, 1101.4027

Walter F, Bolatto AD, Leroy AK, Veilleux S, Warren SR, Hodge J, Levy RC, Meier DS, Ostriker EC, Ott J, Rosolowsky E, Scoville N, Weiss A, Zschaechner L, Zwaan M (2017) Dense Molecular Gas Tracers in the Outflow of the Starburst Galaxy NGC 253. ApJ835(2):265, DOI 10.3847/1538-4357/835/2/265, 1701.05040

Wang T, Yang C, Wang H, Ferland G (2015) Evidence for Photoionization-driven Broad Absorption Line Variability. ApJ814(2):150, DOI 10.1088/0004-637X/814/2/150, 1510.04945

Wang X, Loeb A (2016) Contribution of quasar-driven outflows to the extragalactic gamma-ray background. Nature Physics 12:1116-1118, DOI 10.1038/nphys3837

Wang X, Loeb A (2017) Ultrahigh energy cosmic rays from nonrelativistic quasar outflows. PRD95(6):063007, DOI 10.1103/PhysRevD.95.063007, 1611.07616

Wang X, Loeb A (2018) Self-sustaining Star Formation Fronts in Filaments during the Cosmic Dawn. ApJLett862(2):L14, DOI 10.3847/2041-8213/aad3ce, 1804.02407

Weiner BJ, Willmer CNA, Faber SM, Melbourne J, Kassin SA, Phillips AC, Harker J, Metevier AJ, Vogt NP, Koo DC (2006) A Survey of Galaxy Kinematics to $z \sim 1$ in the TKRS/GOODS-N Field. I. Rotation and Dispersion Properties. ApJ653(2):1027-1048, DOI 10.1086/508921, astro-ph/0609090

Weiner BJ, Coil AL, Prochaska JX, Newman JA, Cooper MC, Bundy K, Conselice CJ, Dutton AA, Faber SM, Koo DC, Lotz JM, Rieke GH, Rubin KHR (2009) Ubiquitous Outflows in DEEP2 Spectra of StarForming Galaxies at $\mathrm{z}=1.4$. ApJ692(1):187-211, DOI 10.1088/0004-637X/692/1/187, 0804 . 4686

Weingartner JC, Draine BT (2001) Dust Grain-Size Distributions and Extinction in the Milky Way, Large Magellanic Cloud, and Small Magellanic Cloud. ApJ548(1):296-309, DOI 10.1086/318651, astro-ph/0008146

Weiß A, Neininger N, Hüttemeister S, Klein U (2001) The effect of violent star formation on the state of the molecular gas in M 82. A\&A365:571-587, DOI 10.1051/0004-6361:20000145, astro-ph/0010541

Weiß A, Henkel C, Downes D, Walter F (2003) Gas and dust in the Cloverleaf quasar at redshift 2.5. A\&A409:L41-L45, DOI 10.1051/0004-6361:20031337, astro-ph/0309048

Weiß A, Downes D, Henkel C, Walter F (2005a) Atomic carbon at redshift 2.5. A\&A429:L25-L28, DOI 10.1051/0004-6361:200400085, astro-ph/0409711

Weiß A, Walter F, Scoville NZ (2005b) The spectral energy distribution of CO lines in M 82. 
A\&A438(2):533-544, DOI 10.1051/0004-6361:20052667, astro-ph/0504377

Weiß A, Downes D, Neri R, Walter F, Henkel C, Wilner DJ, Wagg J, Wiklind T (2007) Highly-excited CO emission in APM 08279+5255 at $\mathrm{z}=3.9$. A\&A467(3):955-969, DOI 10.1051/0004-6361:20066117, astro-ph/0702669

Weiß A, Walter F, Downes D, Carrili CL, Henkel C, Menten KM, Cox P (2012) On the Variations of Fundamental Constants and Active Galactic Nucleus Feedback in the Quasi-stellar Object Host Galaxy RXJ0911.4+0551 at $z=2.79$. ApJ753(2):102, DOI 10.1088/0004-637X/753/2/102, 1204.5614

Welty DE, Ritchey AM, Dahlstrom JA, York DG (2014) Diffuse Interstellar Bands versus Known Atomic and Molecular Species in the Interstellar Medium of M82 toward SN 2014J. ApJ792(2):106, DOI 10.1088/0004-637X/792/2/106, 1404.2639

Werk JK, Prochaska JX, Tumlinson J, Peeples MS, Tripp TM, Fox AJ, Lehner N, Thom C, O’Meara JM, Ford AB, Bordoloi R, Katz N, Tejos N, Oppenheimer BD, Davé R, Weinberg DH (2014) The COSHalos Survey: Physical Conditions and Baryonic Mass in the Low-redshift Circumgalactic Medium. ApJ792(1):8, DOI 10.1088/0004-637X/792/1/8, 1403.0947

Werk JK, Rubin KHR, Bish HV, Prochaska JX, Zheng Y, O’Meara JM, Lenz D, Hummels C, Deason AJ (2019) The Nature of Ionized Gas in the Milky Way Galactic Fountain. arXiv e-prints arXiv: $1904.11014,1904.11014$

Westmoquette MS, Smith LJ, Gallagher I J S (2011) Spatially resolved optical integral field unit spectroscopy of the inner superwind of NGC 253. MNRAS414(4):3719-3739, DOI 10.1111/j.1365-2966. 2011.18675.x, 1103.1775

Whittle M (1985) The narrow line region of active galaxies - I. Nuclear O III profiles. MNRAS213:1, DOI 10.1093/mnras/213.1.1

Wiener J, Oh SP, Zweibel EG (2017) Interaction of cosmic rays with cold clouds in galactic haloes. MNRAS467(1):646-660, DOI 10.1093/mnras/stx 109, 1610.02041

Wilson TL, Rood R (1994) Abundances in the Interstellar Medium. ARA\&A32:191-226, DOI 10.1146/ annurev.aa.32.090194.001203

Winkler PF, Smith RC, Points SD, MCELS Team (2015) The Interstellar Medium in the Small Magellanic Cloud: Results from MCELS. In: Points S, Kunder A (eds) Fifty Years of Wide Field Studies in the Southern Hemisphere: Resolved Stellar Populations of the Galactic Bulge and Magellanic Clouds, Astronomical Society of the Pacific Conference Series, vol 491, p 343

Wisotzki L, Bacon R, Blaizot J, Brinchmann J, Herenz EC, Schaye J, Bouché N, Cantalupo S, Contini T, Carollo CM, Caruana J, Courbot JB, Emsellem E, Kamann S, Kerutt J, Leclercq F, Lilly SJ, Patrício V, Sandin C, Steinmetz M, Straka LA, Urrutia T, Verhamme A, Weilbacher PM, Wendt M (2016) Extended Lyman $\alpha$ haloes around individual high-redshift galaxies revealed by MUSE. A\&A587:A98, DOI 10.1051/0004-6361/201527384, 1509.05143

Wisotzki L, Bacon R, Brinchmann J, Cantalupo S, Richter P, Schaye J, Schmidt KB, Urrutia T, Weilbacher PM, Akhlaghi M, Bouché N, Contini T, Guiderdoni B, Herenz EC, Inami H, Kerutt J, Leclercq F, Marino RA, Maseda M, Monreal-Ibero A, Nanayakkara T, Richard J, Saust R, Steinmetz M, Wendt M (2018) Nearly all the sky is covered by Lyman- $\alpha$ emission around high-redshift galaxies. Nature562(7726):229-232, DOI 10.1038/s41586-018-0564-6, 1810.00843

Wolfe AM, Gawiser E, Prochaska JX (2005) Damped Ly $\alpha$ Systems. ARA\&A43(1):861-918, DOI 10 1146/annurev.astro.42.053102.133950, astro-ph/0509481

Wolfire MG, Hollenbach D, McKee CF, Tielens AGGM, Bakes ELO (1995) The Neutral Atomic Phases of the Interstellar Medium. ApJ443:152, DOI 10.1086/175510

Wolfire MG, McKee CF, Hollenbach D, Tielens AGGM (2003) Neutral Atomic Phases of the Interstellar Medium in the Galaxy. ApJ587(1):278-311, DOI 10.1086/368016, astro-ph/0207098

Woo JH, Son D, Bae HJ (2017) Delayed or No Feedback? Gas Outflows in Type 2 AGNs. III. ApJ839(2):120, DOI 10.3847/1538-4357/aa6894, 1702.06681

Wright T (1750) An original theory or new hypothesis of the universe : founded upon general phaenomena of the visible creation; and particularly the Via the laws of nature, and solving by mathematical principles : the Lactea ...compris'd in nine familiar letters from the author to his friendand : illustrated with upward of thirty graven and mezzotinto plates ... DOI 10.3931/e-rara-28672

Wylezalek D, Morganti R (2018) Questions and challenges of what powers galactic outflows in active galactic nuclei. Nature Astronomy 2:181-182, DOI 10.1038/s41550-018-0409-0, 1802 . 10307

Wylezalek D, Flores AM, Zakamska NL, Greene JE, Riffel RA (2019) Ionised gas outflow signatures in SDSS-IV MaNGA active galactic nuclei. arXiv e-prints arXiv:1911.10212, 1911.10212

Xie Y, Ho LC, Li A, Shangguan J (2018) The Widespread Presence of Nanometer-size Dust Grains in the Interstellar Medium of Galaxies. ApJ867(2):91, DOI 10.3847/1538-4357/aae2b0, 1809.06552 
Xu X, Arav N, Miller T, Benn C (2018) A Mini-BAL Outflow at 900 pc from the Central Source: VLT/Xshooter Observations. ApJ858(1):39, DOI 10.3847/1538-4357/aab7ea, 1805.01545

Yamagishi M, Kaneda H, Ishihara D, Kondo T, Onaka T, Suzuki T, Minh YC (2012) AKARI near-infrared spectroscopy of the aromatic and aliphatic hydrocarbon emission features in the galactic superwind of M 82. A\&A541:A10, DOI 10.1051/0004-6361/201218904, 1203.2794

Yang B, Stancil PC, Balakrishnan N, Forrey RC (2010) Rotational Quenching of CO due to $\mathrm{H}_{2}$ Collisions. ApJ718(2):1062-1069, DOI 10.1088/0004-637X/718/2/1062, 1004.3923

Yang HYK, Ruszkowski M, Ricker PM, Zweibel E, Lee D (2012) The Fermi Bubbles: Supersonic Active Galactic Nucleus Jets with Anisotropic Cosmic-Ray Diffusion. ApJ761(2):185, DOI 10.1088/ 0004-637X/761/2/185, 1207.4185

Yesuf HM, Koo DC, Faber SM, Prochaska JX, Guo Y, Liu FS, Cunningham EC, Coil AL, Guhathakurta P (2017) No Evidence for Feedback: Unexceptional Low-ionization Winds in Host Galaxies of Low Luminosity Active Galactic Nuclei at Redshift $z \sim 1$. ApJ841(2):83, DOI 10.3847/1538-4357/aa6fae, 1704.08348

Yoshida M, Kawabata KS, Ohyama Y (2011) Spectropolarimetry of the Superwind Filaments of the Starburst Galaxy M 82: Kinematics of Dust Outflow. PASJ63:493, DOI 10.1093/pasj/63.sp2.S493, 1012.1503

Yoshida M, Kawabata KS, Ohyama Y, Itoh R, Hattori T (2019) Spectropolarimetry of the superwind filaments of the starburst galaxy M 82 II: Kinematics of the dust surrounding the nuclear starburst ${ }^{y}$. PASJ71(4):87, DOI 10.1093/pasj/psz069, 1905. 11897

Yuan F, Narayan R (2014) Hot Accretion Flows Around Black Holes. ARA\&A52:529-588, DOI 10.1146/ annurev-astro-082812-141003, 1401.0586

Yuan F, Gan Z, Narayan R, Sadowski A, Bu D, Bai XN (2015) Numerical Simulation of Hot Accretion Flows. III. Revisiting Wind Properties Using the Trajectory Approach. ApJ804(2):101, DOI 10.1088/ 0004-637X/804/2/101, 1501.01197

Yun MS, Ho PTP, Lo KY (1994) A high-resolution image of atomic hydrogen in the M81 group of galaxies. Nature372(6506):530-532, DOI 10.1038/372530a0

Yusef-Zadeh F, Hewitt JW, Arendt RG, Whitney B, Rieke G, Wardle M, Hinz JL, Stolovy S, Lang CC, Burton MG, Ramirez S (2009) Star Formation in the Central $400 \mathrm{pc}$ of the Milky Way: Evidence for a Population of Massive Young Stellar Objects. ApJ702(1):178-225, DOI 10.1088/0004-637X/702/ $1 / 178,0905.2161$

Yusef-Zadeh F, Wardle M, Heywood I, Cotton W, Royster M (2019) Cosmic-ray Particles in the Galactic Center: Blowing in the Wind. arXiv e-prints arXiv:1912.11057, 1912.11057

Zabl J, Bouché NF, Schroetter I, Wendt M, Finley H, Schaye J, Conseil S, Contini T, Marino RA, Mitchell P, Muzahid S, Pezzulli G, Wisotzki L (2019) MusE GAs FLOw and Wind (MEGAFLOW) II. A study of gas accretion around $\mathrm{z} \approx 1$ star-forming galaxies with background quasars. MNRAS485(2):19611980, DOI 10.1093/mnras/stz392, 1901.11416

Zahedy FS, Chen HW, Rauch M, Wilson ML, Zabludoff A (2016) Probing the cool interstellar and circumgalactic gas of three massive lensing galaxies at $z=0.4-0.7$. MNRAS458(3):2423-2442, DOI $10.1093 / \mathrm{mnras} / \mathrm{stw} 484,1510.04307$

Zahedy FS, Chen HW, Johnson SD, Pierce RM, Rauch M, Huang YH, Weiner BJ, Gauthier JR (2019) Characterizing circumgalactic gas around massive ellipticals at $z \sim 0.4$ - II. Physical properties and elemental abundances. MNRAS484(2):2257-2280, DOI 10.1093/mnras/sty3482, 1809.05115

Zakamska NL, Schmidt GD, Smith PS, Strauss MA, Krolik JH, Hall PB, Richards GT, Schneider DP, Brinkmann J, Szokoly GP (2005) Candidate Type II Quasars from the Sloan Digital Sky Survey. III. Spectropolarimetry Reveals Hidden Type I Nuclei. AJ129(3):1212-1224, DOI 10.1086/427543, astro-ph/0410054

Zaritsky D (1994) Preliminary Evidence for Dust in Galactic Halos. AJ108:1619, DOI 10.1086/117182

Zech WF, Lehner N, Howk JC, Dixon WVD, Brown TM (2008) The High-Velocity Gas toward Messier 5 : Tracing Feedback Flows in the Inner Galaxy. ApJ679(1):460-480, DOI 10.1086/587135, 0802 . 0286

Zhang D, Davis SW (2017) Radiation Hydrodynamic Simulations of Dust-driven Winds. ApJ839(1):54, DOI 10.3847/1538-4357/aa6935, 1612.00022

Zhang D, Davis SW, Jiang YF, Stone JM (2018) Dusty Cloud Acceleration by Radiation Pressure in Rapidly Star-forming Galaxies. ApJ854(2):110, DOI 10.3847/1538-4357/aaa8e4, 1708 . 02946

Zhao JH, Morris MR, Goss WM, An T (2009) Dynamics of Ionized Gas at the Galactic Center: Very Large Array Observations of the Three-dimensional Velocity Field and Location of the Ionized Streams in Sagittarius A West. ApJ699(1):186-214, DOI 10.1088/0004-637X/699/1/186, 0904 . 3133

Zheng W, Wang JX, Kriss GA, Sahnow D, Allen M, Dopita M, Tsvetanov Z, Bicknell G (2008) Spatially 
Resolved Far-Ultraviolet Spectroscopy of the Nuclear Region of NGC 1068. ApJ686(2):881-891, DOI 10.1086/591547, 0806.4641

Zheng Y, Peek JEG, Putman ME, Werk JK (2019) Revealing the Milky Way's Hidden Circumgalactic Medium with the Cosmic Origins Spectrograph Quasar Database for Galactic Absorption Lines. ApJ871(1):35, DOI 10.3847/1538-4357/aaf6eb, 1710.10703

Zhu G, Ménard B (2013) Calcium H \&amp; K Induced by Galaxy Halos. ApJ773(1):16, DOI 10.1088/ $0004-637 X / 773 / 1 / 16,1304.0451$

Zhu GB, Comparat J, Kneib JP, Delubac T, Raichoor A, Dawson KS, Newman J, Yèche C, Zhou X, Schneider DP (2015) Near-ultraviolet Spectroscopy of Star-forming Galaxies from eBOSS: Signatures of Ubiquitous Galactic-scale Outflows. ApJ815(1):48, DOI 10.1088/0004-637X/815/1/48, 1507.07979

Zhukovska S, Dobbs C, Jenkins EB, Klessen RS (2016) Modeling Dust Evolution in Galaxies with a Multiphase, Inhomogeneous ISM. ApJ831(2):147, DOI 10.3847/0004-637X/831/2/147, 1608.04781

Zovaro HRM, Nesvadba NPH, Sharp R, Bicknell GV, Groves B, Mukherjee D, Wagner AY (2019) Searching for signs of jet-driven negative feedback in the nearby radio galaxy UGC 05771. MNRASp 2119, DOI 10.1093/mnras/stz2459, 1909.00144

Zschaechner LK, Walter F, Bolatto A, Farina EP, Kruijssen JMD, Leroy A, Meier DS, Ott J, Veilleux S (2016) The Molecular Wind in the Nearest Seyfert Galaxy Circinus Revealed by ALMA. ApJ832(2):142, DOI 10.3847/0004-637X/832/2/142, 1609.06316

Zschaechner LK, Bolatto AD, Walter F, Leroy AK, Herrera C, Krieger N, Kruijssen JMD, Meier DS, Mills EAC, Ott J, Veilleux S, Weiss A (2018) Spatially Resolved ${ }^{12} \mathrm{CO}(2-1) /{ }^{12} \mathrm{CO}(1-0)$ in the Starburst Galaxy NGC 253: Assessing Optical Depth to Constrain the Molecular Mass Outflow Rate. ApJ867(2):111, DOI 10.3847/1538-4357/aadf32, 1809.01160

Zubko VG, Laor A (2000) The Spectral Signature of Dust Scattering and Polarization in the Near-Infrared to Far-Ultraviolet. I. Optical Depth and Geometry Effects. ApJSupp.128(1):245-269, DOI 10.1086/ 313373 , astro-ph/9905294

Zubovas K (2018) Massive outflow properties suggest AGN fade slowly. MNRAS473(3):3525-3535, DOI $10.1093 / \mathrm{mnras} / \mathrm{stx} 2569,1710.01053$

Zubovas K, King A (2012) Clearing Out a Galaxy. ApJLett745(2):L34, DOI 10.1088/2041-8205/745/2/ L34, 1201.0866

Zubovas K, King AR (2014) Galaxy-wide outflows: cold gas and star formation at high speeds. MNRAS439(1):400-406, DOI 10.1093/mnras/stt2472, 1401.0392

Zubovas K, King AR, Nayakshin S (2011) The Milky Way's Fermi bubbles: echoes of the last quasar outburst? MNRAS415(1):L21-L25, DOI 10.1111/j.1745-3933.2011.01070.x, 1104. 5443

Zubovas K, Nayakshin S, Sazonov S, Sunyaev R (2013) Outflows of stars due to quasar feedback. MNRAS431(1):793-798, DOI 10.1093/mnras/stt214, 1302.0999 